\title{
Stabilisation of Natural Colourants and Lignocellulosic Textiles
}

\author{
by
}

\section{Ying Tang}

\author{
A thesis \\ submitted to the Victoria University of Wellington \\ in fulfilment of the requirements for the degree of \\ Doctor of Philosophy \\ in Chemistry
}

Victoria University of Wellington

2011 



\section{Abstract}

The lignocellulosic fibres extracted from the leaves of New Zealand flax, Phormium tenax, have been used as the principal textile fibre by Maori since preEuropean times. Variations of antifungal activity were observed in Phormium fibres of different cultivars. The most resistant cultivars of $P$. tenax in an aqueous antifungal assay also possessed the greatest variety of naturally-occurring 7hydroxycoumarins as identified by mass spectroscopy, ESI-MS.

In addition to antifungal effects, coumarins function as fluorescent whitening agents in Phormium fibres and play a role in the fibre's photodegradation. Ultraviolet irradiation $(350-400 \mathrm{~nm})$ of the fibre resulted in a substantial loss of the blue fluorescence originating from a number of 7-hydroxycoumarins present, together with the formation of new fluorophores absorbing and emitting at longer wavelengths, which contribute to the photoyellowing of the fibre. The photolysis of two standard 7-hydroxycoumarins in aqueous solution was examined and two primary photoproducts were elucidated by ESI-MS: a photodimer containing a linking cyclobutane ring and a monomeric photooxidation product. The formation of at least some of the photoproducts is associated with the coumarin-sensitised generation of reactive oxygen species, hydrogen peroxide and superoxide.

The fluorescence properties and photodegradation of Chinese handmade papers were also investigated. Papers manufactured by traditional methods were found to be more photostable than that produced from chemically-facilitated techniques.

Key Words: Phormium tenax, antifungal agent, fluorescent whitening agent, ESI-MS, 7-hydroxycoumarin, photoyellowing, photodimerisation, reactive oxygen species, Chinese handmade paper 


\section{List of Publications}

\section{Peer Reviewed Journal Articles:}

1. Smith, G. J., Tang, Y., Dyer, J. M. and Scheele, S. M., Coumarins in Phormium (New Zealand Flax) Fibers: Their Role in Fluorescence and Photodegradation. Photochemistry and Photobiology 2011, 87: 45-50

\section{Peer Reviewed Conferences:}

2. Tang, Y., Smith, G. J., Dyer, J. M. and Scheele, S. M., A double-edged sword: coumarins in the photodegradation and fungal deterioration of the Phormium fibres. The 2010 International Chemical Congress of Pacific Basin Societies, Honolulu, US, Dec. 15-20, 2010 (poster presentation)

3. Tang, Y., Smith, G. J., Dyer, J. M. and Scheele, S. M., A double-edged sword: coumarins in the photodegradation and fungal deterioration of the Phormium fibres. The $6^{\text {th }}$ Asian Photochemistry Conference, Wellington, NZ, Nov. 14-18, 2010 (poster presentation)

4. Smith, G. J., Weston, R. J., Dyer, J. M. and Tang, Y. Photoproducts of some natural coumarins in water. The $6^{\text {th }}$ Asian Photochemistry Conference, Wellington, NZ, Nov. 14-18, 2010 


\section{Acknowledgement}

First and foremost, my sincere and heartfelt gratitude goes to A/Prof. Gerald Smith, my supervisor, for being patient, insightful, approachable and helpful throughout this project; and I truly could not have finished my $\mathrm{PhD}$ without his constant motivation, guidance and support.

I am deeply indebted to Dr. Jolon Dyer (Agresearch, Lincoln) and Dr. Roderick Weston for their expertise and significant contribution to the ESI-mass spectral analysis and data interpretation. They were also kind enough to perform some of the proofreading for this thesis.

A special thanks to Sue Scheele (Landcare Research, Lincoln) for generously providing the Phormium specimens and reviewing the mycological chapter. I want to thank Katarina Tawiri, the staff of Landcare Research Experimental Garden, who hand-stripped the fibres from cultivated plants.

I gratefully acknowledge the work of several other research fellows of the group for their previous research on Phormium fibres: Dr. Peter Roberts, Dr. John Patterson, Stephen Williams and Sarah Wilcox. I am immensely grateful for their knowledge, openness, and enthusiasm.

I was also helped tremendously by the staff of the School of Chemical and Physical Sciences: Prof. John Spencer, Dr. Richard Tilley, David Flynn, Teresa Gen, Jackie King, Alan Rennie, as well as my colleagues, Hui Lin Tan, Sherry Xu, Teck Lim, Ishira Samarasinghe and many other more who gave me support and encouragement in one way or another.

I extend a very special thanks to the staff of the Department of Archaeology and Scientific History, Graduate University of Chinese Academy of Sciences, China, who treated me as an honoured guest in Beijing, 2008, and generously provided 
me with the Chinese paper specimens.

Furthermore, I wish to acknowledge the financial support from Vice Chancellor's Strategic Doctoral Scholarship and other grants provided by Victoria University of Wellington; without which I would not be able to finish this work.

Finally, I am forever indebted to my family and friends for their love, understanding and encouragement. My parents, who I left behind in China, are always in the warmest part of my heart.

Kia ora and Xie-xie.

Ying Tang

December, 2010 


\section{Table of Contents}

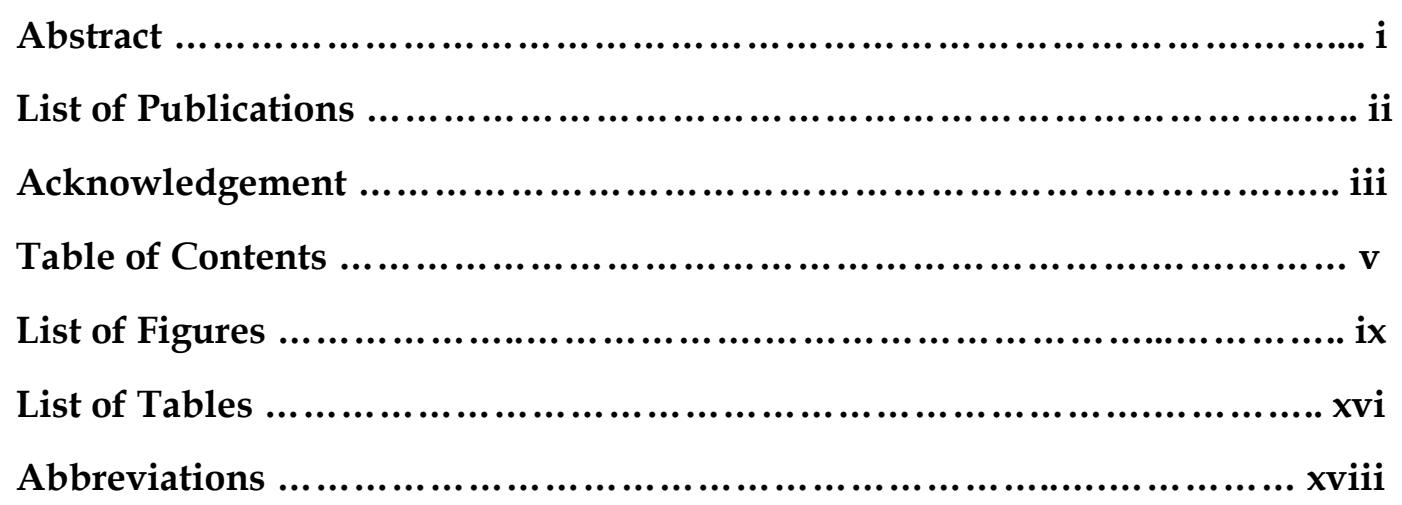

\section{Chapter 1: Introduction}

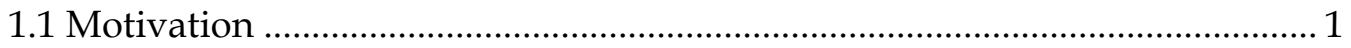

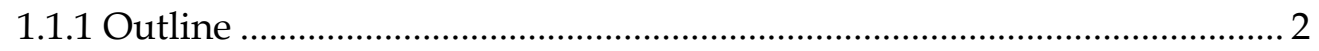

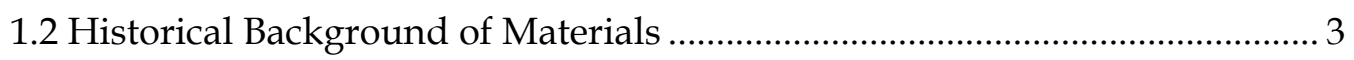

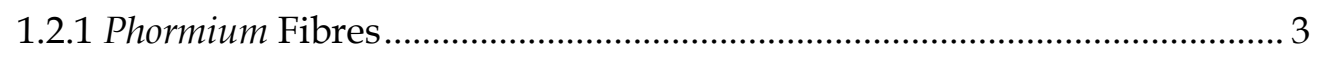

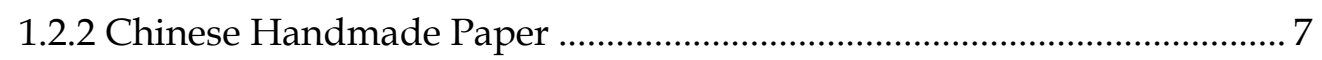

Chapter 2: Mycological Screening of Phormium Fibres

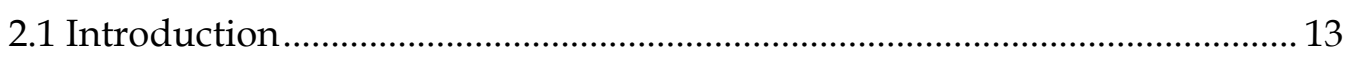

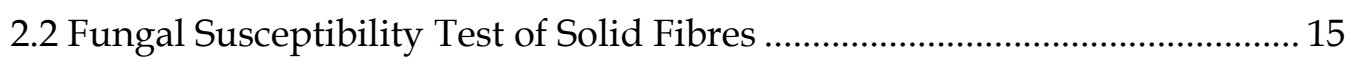

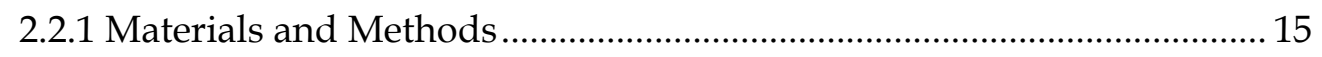

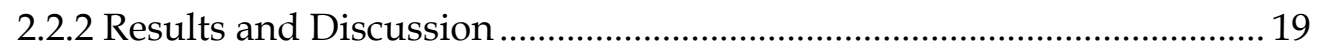

2.3 Antifungal Assay of Aqueous Extracts........................................................... 23

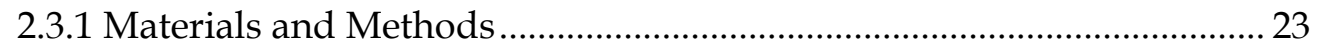

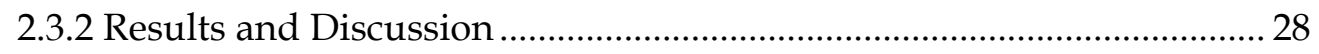

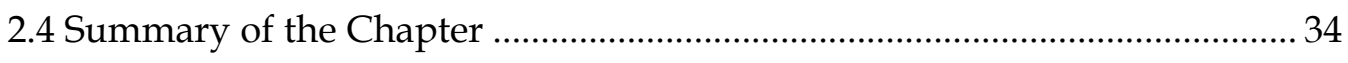




\section{Chapter 3: Identifying Fluorescent Species in Phormium Fibres}

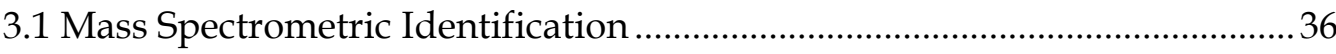

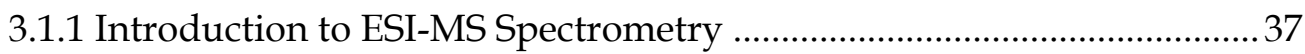

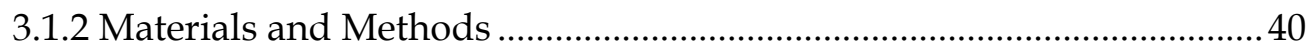

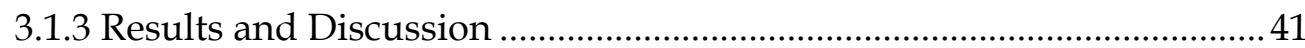

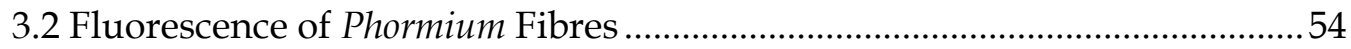

3.2.1 Electronic Excited States and Fluorescence...........................................54

3.2.1 Coumarins and Fluorescence of Phormium Fibres ..................................57

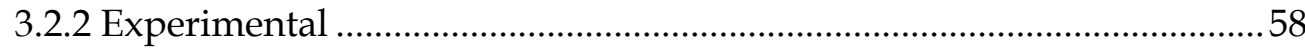

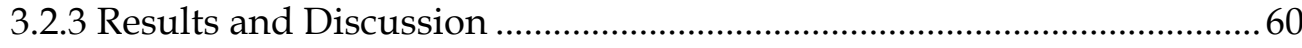

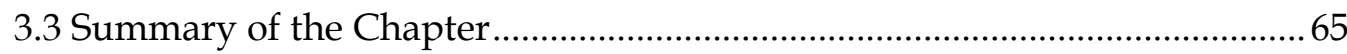

\section{Chapter 4: Photodegradation of Phormium Fibres: Coumarin Photochemistry}

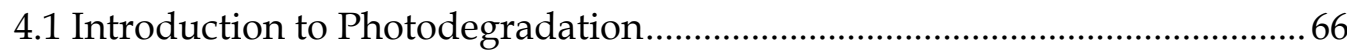

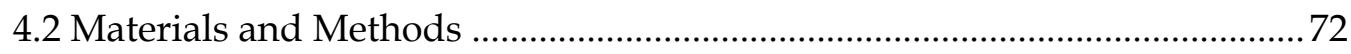

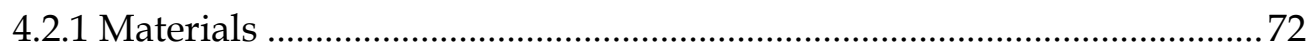

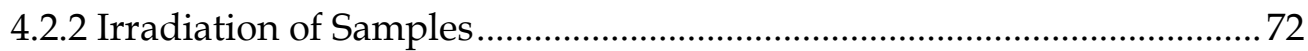

4.2.3 Spectroscopic Characterisations ………….............................................. 74

4.2.4 Superoxide and Peroxide Assays ..............................................................75

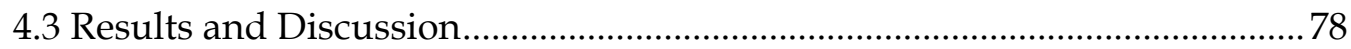

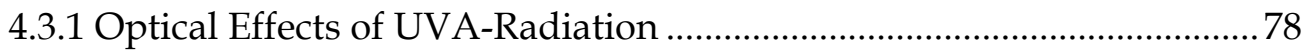

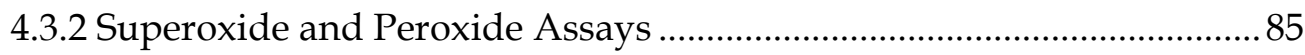

4.3.3 Mass Spectrometrical Photoproduct Analysis......................................... 92

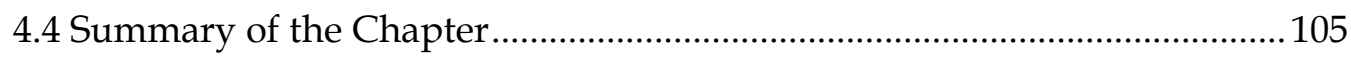

Chapter 5: Chinese Handmade Paper: Fluorescence and Photodegradation

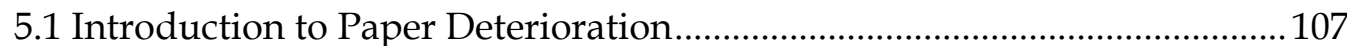




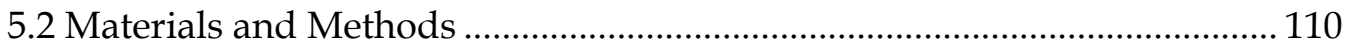

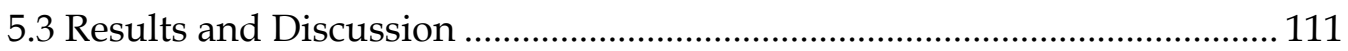

5.3.1 Fluorescence of Three Traditional Papers................................................. 111

5.3.2 UVA-induced Spectroscopic Changes .................................................. 113

5.3.3 Generation of Peroxide and Superoxide ….......................................... 122

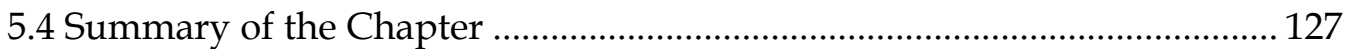

\section{Chapter 6: Microstructural and Elemental Analysis}

6.1 Metal Ions on Textiles: History and Science .................................................. 129

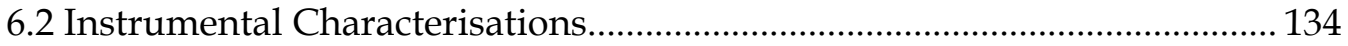

6.2.1 Scanning Electron Microscopy (SEM) ………….................................. 134

6.2.2 X-ray Fluorescence Spectroscopy (XRF)................................................ 136

6.2.3 Flame Atomic Absorption Spectroscopy (FAAS) ................................. 136

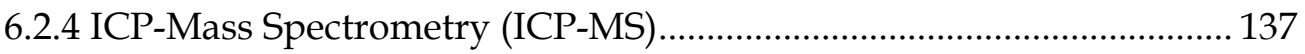

6.3 Results and Discussion: Four Case Studies.................................................. 138

6.3.1 Determining Iron Contents of Phormium Fibres..................................... 138

6.3.2 Traditional Maori Dyeing: Wood-Ash Mordant...................................... 139

6.2.3 Photostability of Alum-Treated Phormium Fibres................................... 150

6.2.4 SEM Investigation of Chinese Handmade Paper..................................... 155

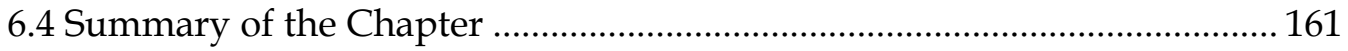

\section{Chapter 7: Closing Discussion and Conclusions}

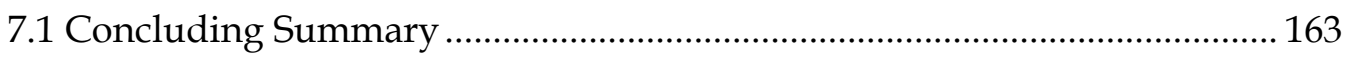

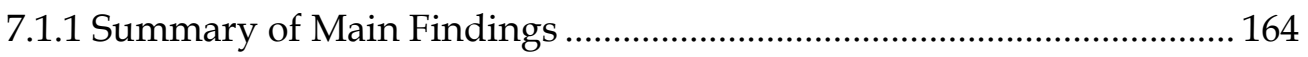

7.2 Limits of Current Study and Future Work ................................................. 167

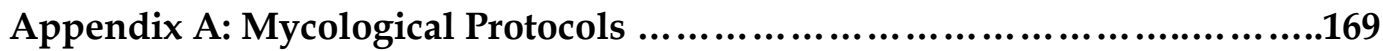

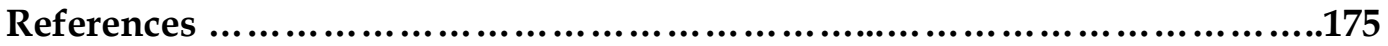




\section{List of Figures}

\section{Chapter One}

Figure 1.1 Plantation of $P$. tenax and the traditional fibre processing method (stripping with a mussel shell).

Figure 1.2 Methods of traditional Chinese papermaking[36,37]: macerated plant fibre (A), sunning of fibre (B), pounding with wooden pestles (C), purifying pulps in running water (D), sheet formation (E) and drying on a heated wall (F). Pictures B to E were taken by Feisong Huang at the Red-Star Xuan papermill in Jing County, Anhui province, China.

\section{Chapter Two}

Figure 2.1 Confocal images of Phormium fibres (green auto-fluorescence at 488 $\mathrm{nm}$ ) inoculated by P. glabrum (red auto-fluorescence at $647 \mathrm{~nm}$ ) after 7 days of incubation. Yellow areas indicate the overlapping of the red and green signals. B is an enlarge view of a degraded fibre in contact with fungal mycelia.

Figure 2.2 Fungal susceptibility test of fibres from four Phormium cultivars (rows from top to bottom are: Awahou, Whareongaonga, Potaka and Tapamangu). Columns 1 to 3 (left-hand side) are controls; columns 4 to 6 (right-hand side) are inoculums with P. glabrum ( $400 \mu \mathrm{L} \times 500$ CFUs. $\left.\mathrm{mL}^{-1}\right)$. A is the original image. B is the interactive graphic interface of Dot Blot Analyzer for ImageJ after 8-bit conversion

Figure 2.3 Colour variations of Phormium fibres on inoculation of P. glabrum (A) and A. wentii (B), inoculum: 2000 CFUs per $0.1 \mathrm{~g}$ of fibre, 7 days of incubation... 20

Figure 2.4 Fungal-induced colour change between controls and inoculums (incremental MID or IMID) of Phormium fibres from 14 cultivars

Figure 2.5 Structures of coumarins used in this study .24

Figure 2.6 Principle of resazurin (sodium salt) in the colorimetric assay .25

Figure 2.7 An inhibition titration against fungal broth of P. glabrum $(10,000$ CFUs. $\mathrm{mL}^{-1}, 48 \mathrm{hrs}$ of incubation) as in absorption spectra (A) and as a calibration 
curve of peak ratio $601 \mathrm{~nm}: 573 \mathrm{~nm}(\mathrm{~B})$

Figure 2.8 Absorption spectra of resazurin-dyed MIC cultures of amphotericin B (AmpB) inoculated with $P$. glabrum $\left(0.4 \times 10^{4}\right.$ to $5 \times 10^{4}$ CFUs. $\left.\mathrm{mL}^{-1}\right)$.

Figure 2.9 Absorption spectra of resazurin-dyed MIC cultures of amphotericin B (AmpB), coumarin (COU) and 7-hydroxycoumarin (UMB) inoculated with $P$. glabrum $\left(0.4 \times 10^{4}\right.$ to $\left.5 \times 10^{4} \mathrm{CFUs} \cdot \mathrm{mL}^{-1}\right)$...

Figure 2.10 Absorption spectra of resazurin-dyed cultures of aqueous (left column) and DCM (right column) extracts from fibres of three cultivars of $P$. tenax against $P$. glabrum $(\mathrm{A}$ and $\mathrm{B})$ and $A$. wentii $(\mathrm{C}$ and $\mathrm{D})$. Inoculum viability was within the range of $0.4 \times 10^{4}$ to $5 \times 10^{4} \mathrm{CFUs} \cdot \mathrm{mL}^{-1}$.

Figure 2.11 Antifungal activities (shown as 573 / $601 \mathrm{~nm}$ peak ratio) of $3 \mathrm{DCM}$ and 14 aqueous extracts of Phormium fibres against P. glabrum (A) and A. wentii (B). Inoculum viability was within the range of $0.4 \times 10^{4}$ to $5 \times 10^{4} \mathrm{CFUs} \cdot \mathrm{mL}^{-1} \ldots . .32$

\section{Chapter Three}

Figure 3.1 Schematic of electrospray ionisation and the desolvation process ...... 37

Figure 3.2 Schematic diagram of ESI tandem MS (ESI-MS/MS) and the main processes in the scan modes of MS and MS/MS. CID stands for collision-induced dissociation, as occurs when an inert argon gas is present in the collision cell.... 39

Figure 3.3 ESI-MS spectra of standard furfural (A), coumarin (B), 7hydroxycoumarin (C), 4-methyl-7-hydroxycoumarin (D), 6,7-dihydroxycoumarin (E) and 7-hydroxy-6-methoxycoumarin (F), with target molecular ions marked in red.

Figure 3.4 ESI-MS spectra of aqueous extracts from Phormium fibres of cultivars Mawaru (A), Ruawai (B) and Tapmangu (C).

Figure 3.5 ESI-MS spectra of aqueous extracts from Phormium fibres of cultivars Arawa (A), Ngaro (B) and Potaka (C).

Figure 3.6 ESI-MS/MS spectra of standard furfural (top) and the aqueous extract of Mawaru (bottom), with characteristic fragments labelled. .46

Figure 3.7 ESI-MS/MS spectra of standard coumarin (top) and the aqueous extract of Mawaru (bottom), with characteristic fragments labelled

Figure 3.8 ESI-MS/MS spectra of 7-hydroxycoumarin (top) and the aqueous 
extract of Mawaru (bottom), with characteristic fragments labelled 48

Figure 3.9 ESI-MS/MS spectra of 7-hydroxy-4-methylcoumarin (top) and the aqueous extract of Mawaru (bottom), with characteristic fragments labelled.

Figure 3.10 ESI-MS/MS spectra of 6,7-dihydroxycoumarin (top) and the aqueous extract of Mawaru (bottom), with characteristic fragments labelled. 50

Figure 3.11 ESI-MS/MS spectrum of 7-hydroxy-6-methoxycoumarin and aqueous extract of Mawaru (bottom), with characteristic fragments labelled......51

Figure 3.12 A Jablonski diagram in which the molecular energetic levels are depicted by horizontal lines, non-radiative transitions are indicated by wave arrows and radiative transitions by straight arrows.

Figure 3.13 Generic structure of coumarins (R1 - $\mathrm{R} 6=\mathrm{H}, \mathrm{OH}, \mathrm{OCH}_{3}$ or $\left.\mathrm{CH}_{3}\right) \ldots .58$

Figure 3.14 3D-fluorescence spectra of aqueous solutions of four 7hydroxycoumarins: 7-hydroxycoumarin (A), 4-methyl-7-hydroxycoumarin (B), 6,7-dihydroxycoumarin (C) and 7-hydroxy-6-methoxycoumarin (D) at different $\mathrm{pHs}$ from acidic to alkaline. Contour intervals represent $1 / 10$ th of the maximum fluorescence of the three plots of a sample.

Figure 3.15 3D-fluorescence spectra of solid Phormium fibres of five cultivars: Arawa (A), Ngaro (B), Tapamangu (C), Mawaru (D) and Potaka (E).

Figure 3.16 3D-fluorescence spectra of aqueous extracts from Phormium fibres of five cultivars: Arawa (A), Tapamangu (B), Ngaro (C), Mawaru (D) and Potaka (E), under acidic, neutral and alkaline conditions. .64

\section{Chapter Four}

Figure 4.1 Two major mechanisms of photosensitised formation of ROS: type I (cyan for the hydrogen transfer scheme; blue for the electron transfer scheme) and type II (green). Fenton reactions are marked in red[99]

Figure 4.2 The absolute spectral distribution of UVA irradiance on a sample positioned $1 \mathrm{~cm}$ away from a $6 \mathrm{~W}$, NEC 'blacklight' fluorescent UV lamp. .73

Figure 4.3 Standard curve of $\left[\mathrm{H}_{2} \mathrm{O}_{2}\right]$ in the range of 0 to $4.0 \mu \mathrm{M}$. Data were the mean of triplicate determinations.

Figure 4.4 Reflectance spectra of solid fibres of five cultivars of $P$. tenax before (black curve) and after 24 hrs of UVA irradiation under dry (red curve) or wet 
conditions (blue curve): Arawa (A), Ngaro (B), Tapamangu (C), Mawaru (D) and Potaka (E).

Figure 4.5 3D-fluorescence spectra of solid fibres of five cultivars of $P$. tenax before (left side) and after 24 hrs of UVA irradiation, dry (middle) or wet (right side): Arawa (A), Ngaro (B), Tapamangu (C), Mawaru (D) and Potaka (E). ......... 81

Figure 4.6 Absorption spectra of saturated aqueous solution of 6,7dihydroxycoumarin (A) and 7-hydroxy-6-methoxycoumarin (B) after UVA irradiation from 0 to $96 \mathrm{hrs}$ at $24 \mathrm{hrs}$ intervals.

Figure 4.7 3D-fluorescence spectra of aqueous solutions of 6,7-

dihydroxycoumarin (esculetin, 20-fold diluted from original solution) and 7hydroxy-6-methoxycoumarin (scopoletin, 800-fold diluted), after UVA irradiation from 0 to 96 hrs at 24 hrs intervals

Figure 4.8 Production of $\mathrm{H}_{2} \mathrm{O}_{2}$ in wet UVA-irradiated Phormium fibres. $\mathrm{H}_{2} \mathrm{O}_{2}$ is shown as concentration $(\mu \mathrm{M})$ in the water from irradiated fibre over exposure time from 0 to $24 \mathrm{hrs}$.

Figure 4.9 The effect of superoxide dismutase (SOD) and catalase (CAT) on the photoformation of $\mathrm{H}_{2} \mathrm{O}_{2}$ by wet UVA-irradiated Phormium fibres of three cultivars: Mawaru (A), Potaka (B) and Tapamangu (C). $\mathrm{H}_{2} \mathrm{O}_{2}$ is shown as concentration $(\mu \mathrm{M})$ in the water from irradiated fibre over exposure time from 0 to $24 \mathrm{hrs}$.

Figure 4.10 Photogeneration of $\mathrm{H}_{2} \mathrm{O}_{2}$ by saturated aqueous solutions of 7hydroxylated coumarins (A) and the effect of superoxide dismutase (SOD) and catalase (CAT) on 6,7-dihydroxycoumarin (esculetin, B) and 7-hydroxy-6methoxycoumarin (scopoletin, $\mathrm{C}$ ). $\mathrm{H}_{2} \mathrm{O}_{2}$ is shown as concentration $(\mu \mathrm{M})$ in the irradiated sample over exposure time from 0 to $4 \mathrm{hrs}$

Figure 4.11 ESI-MS spectra of 6,7-dihydroxycoumarin after $0 \mathrm{hr}$ (A), 8 hrs (B), 16 hrs (C) and 24 hrs (D) of UVA irradiation, with molecular ions of the substrate $\left(\mathrm{MH}^{+}\right)$and photoproducts labelled in red.

Figure 4.12 ESI-MS spectrum of 6,7-dihydroxycoumarin after $48 \mathrm{hrs}$ of UVA irradiation, with molecular ions of the substrate $\left(\mathrm{MH}^{+}\right)$and photoproducts labelled in red.

Figure 4.13 ESI-MS spectra of 7-hydroxy-6-methoxycoumarin after $0 \mathrm{hr}$ (A) $24 \mathrm{hrs}$ (B) and $48 \mathrm{hrs}(\mathrm{C})$ of UVA irradiation, with molecular ions of substrate $\left(\mathrm{MH}^{+}\right)$ and ions of photoproducts labelled in red. 
Figure 4.14 ESI-MS/MS spectra of the mono-oxidised $(\mathrm{M}+\mathrm{O})$ product ion $(\mathrm{A})$ and the hydroxylated $\left(\mathrm{M}+\mathrm{H}_{2} \mathrm{O}\right)$ product ion (B) of 6,7-dihydroxycoumarin, with characteristic fragments labelled (in red if the neutral loss was also observed in unirradiated sample; or in blue for those observed only in A and B). The structures drawn are speculative.

Figure 4.15 ESI-MS/MS spectrum of the m/z 355 product ion from 6,7dihydroxycoumarin, with characteristic fragment ions labelled (in red if the neutral loss was also observed in the unirradiated sample).

Figure 4.16 ESI-MS/MS spectrum of the mono-oxidised $(\mathrm{M}+\mathrm{O})$ product ion of 7hydroxy-6-methoxycoumarin, with characteristic fragment ions labelled (in red if the neutral loss was also observed in the unirradiated sample). The structure of the product is indicative only.

Figure 4.17 ESI-MS/MS spectrum of the m/z 383 product ion of 7-hydroxy-6methoxycoumarin, with characteristic fragment ions labelled (in red if the neutral loss was also observed in unirradiated sample).

Figure 4.18 Putative photodegradation pathway of 7-dihydroxycoumarins $(\mathrm{R}=$ $\mathrm{OH}$ or $\mathrm{OCH}_{3}$ ) in aqueous solution upon UVA irradiation, leading to the formation of mono-oxidation products and photodimers. (" product formed during ESI-mass spectrometry).

\section{Chapter Five}

Figure 5.1 Picture of three types of traditional Chinese handmade paper: Xuan paper (A), Mulberry paper (B) and Bamboo paper (C)

Figure 5.2 3D-fluorescence spectra of three types of traditional Chinese handmade paper (original pHs 6.3 8.0), Xuan paper (A), Mulberry paper (B) and Bamboo paper $(\mathrm{C})$, after treatments with weak acid $(\mathrm{HCl}, \mathrm{pH} 4.0)$ or base $\left(\mathrm{NaHCO}_{3}, \mathrm{pH} 9.0\right)$

Figure 5.3 Reflectance spectra of three Xuan papers before (black curve) and after $24 \mathrm{hrs}$ of UVA irradiation under dry (red curve) or wet (blue curve) conditions: X1 (A), X2 (B) and X3 (C)

Figure 5.4 3D-fluorescence spectra of three Xuan papers before (left side) and after 24 hrs of UVA irradiation, under dry (middle) or wet (right side) conditions: X1 (A), X2 (B) and X3 (C)

Figure 5.5 Reflectance spectra of solid fibres from two Mulberry papers, M1 (A) 
and M2 (B), before (black curve) and after 24 hrs of UVA irradiation under dry (red curve) or wet (blue curve) conditions.

Figure 5.6 3D-fluorescence spectra of two Mulberry papers, M1 (A) and M2 (B), before (left side) and after $24 \mathrm{hrs}$ of UVA irradiation, under dry (middle) or wet (right side) conditions.

Figure 5.7 Reflectance spectra of four Bamboo papers before (black curve) and after 24 hrs of UVA irradiation under dry (red curve) or wet (blue curve) conditions: B1 (A), B2 (B), B3 (C) and B4 (D).

Figure 5.8 3D-fluorescence spectra of four Bamboo papers before (left side) and after 24 hrs of UVA irradiation, under dry (middle) or wet (right side) conditions: B1 (A), B2 (B), B3 (C) and B4 (D). 121

Figure 5.9 Production of $\mathrm{H}_{2} \mathrm{O}_{2}$ in three samples of wet UVA-irradiated Xuan Paper (A) and the effect of superoxide dismutase (SOD) and catalase (CAT): X1 (B), $\mathrm{X} 2(\mathrm{C})$ and $\mathrm{X} 3(\mathrm{D}) . \mathrm{H}_{2} \mathrm{O}_{2}$ is shown as concentration $(\mu \mathrm{M})$ in the water from irradiated paper over exposure time from 0 to $24 \mathrm{hrs}$.

Figure 5.10 Production of $\mathrm{H}_{2} \mathrm{O}_{2}$ in two samples of wet UVA-irradiated Mulberry paper (A) and the effect of superoxide dismutase (SOD) and catalase (CAT) on M1 (B) and M2 (C). $\mathrm{H}_{2} \mathrm{O}_{2}$ is shown as concentration $(\mu \mathrm{M})$ in the water from irradiated paper over exposure time from 0 to $24 \mathrm{hrs}$.

Figure 5.11 Production of $\mathrm{H}_{2} \mathrm{O}_{2}$ in four samples of wet UVA-irradiated Bamboo paper (A) and the effect of superoxide dismutase (SOD) and catalase (CAT) on B1 $(B), B 2(C), B 3(D)$ and $\mathrm{B} 4(\mathrm{E}) . \mathrm{H}_{2} \mathrm{O}_{2}$ is shown as concentration $(\mu \mathrm{M})$ in the water from irradiated paper over exposure time from 0 to $24 \mathrm{hrs}$ 126

\section{Chapter Six}

Figure 6.1 SEI images of wool (A), raw silk (B), cotton (C) and Phormium fibre (D), magnification: $\times 800$.

Figure 6.2 Calibration curve of iron standards and plots of sample elutions..... 138

Figure 6.3 Iron levels (dry weight in ppm) of Phormium fibres from five cultivars and the association with their production of $\mathrm{H}_{2} \mathrm{O}_{2}$ after wet UVA-irradiation. $\mathrm{H}_{2} \mathrm{O}_{2}$ is shown as concentration $(\mu \mathrm{M})$ in the water from irradiated fibre over exposure times from 2 to $24 \mathrm{hrs}$.

Figure 6.4 Bark samples of Tanekaha (A) and Mahoe (B) with an enlarged view showing the growth of lichen. 
Figure 6.5 SEM back-scattered images of Tanekaha-dyed Phormium fibres without (A) and with (B) treatment of Mahoe ash, magnification: $\times 200$. An optical image of each sample is also present in the bottom right corner.

Figure 6.6 X-ray spectra of wood ash of Mahoe bark obtained by EDS (A) and XRF (B) analysis.

Figure 6.7 Backscatter images (BS) of the cross-section of a Phormium fibre treated with wood ash of Mahoe (left column, magnification: $\times 500$ ) and a piece of Mahoe bark (right column, magnification: $\times 200$ ) with the growth of lichen on the right side, with EDS mapping of $\mathrm{Al}, \mathrm{K}$ and $\mathrm{Ca}$

Figure 6.8 Reflectance spectra (A) and Kubelka-Munk transforms (B) of Tanekaha-dyed Phormium fibres with (red) or without (black) rubbing with Mahoe ash, when $\mathrm{pH}$ adjusted to 4.4 (solid curve) or 7.0 (dashed curve).

Figure 6.9 Structures of polyphenolic products identified in the cladodes of Tanekaha and a proposed modanting mechanism of complexing with metal ions (bottom right corner).

Figure 6.10 3D fluorescence spectra of Tanekaha-dyed $P$. tenax fibres: no mordant (A), mordanted with wood ash of Mahoe (B), no mordant $+\mathrm{pH} 7.0$ buffered (C) and wood ash-mordanted $+\mathrm{pH} 4.4$ buffered (D). 149

Figure 6.11 The backscatter image (top right corner) of a $90{ }^{\circ} \mathrm{C}$ alum-treated Phormium fibre with associated EDS spectrum and mapping for Al, K and S....151

Figure 6.12 Reflectance spectra of differently treated Phormium fibres, no treatment (control), treated at room temperature (Alum RT) or at $90^{\circ} \mathrm{C}$ (Alum 90), before (A) and after (B) 24 hrs of UVA irradiation, and the associated KubelkaMunk transforms (C).

Figure 6.13 3D-fluorescence spectra of differently treated Phormium fibres before (left column) and after (right column) 24 hrs of UVA irradiation: control (A), Alum RT (B) and Alum 90 (C). .154

Figure 6.14 SEM backscatter images and EDS spectra of three types of traditional Chinese paper: Xuan paper (A), Mulberry paper (B) and Bamboo paper (C), magnification: $\times 700$ .156

Figure 6.15 SEM Backscatter images (BS, magnification: $\times 700)$ and false colour EDS images of Xuan paper and Mulberry paper, mapping for Al, Si and Ca.....158

Figure 6.16 EDS spot analysis of Xuan paper, BS image, magnification: $\times 8000$ for 
the image on the left and $\times 16,000$ for the image on the right

Figure 6.17 Back-scattered image of a Pingao grass with the associated EDS spectrum and false-colour mapping for $\mathrm{Al}$ and $\mathrm{Si}$; magnification: $\times 70$. An optical image of a portion of the grass is also shown in the top right corner. 160

\section{Appendix A}

Figure A1. Loading of fibre discs into 24-well plate and the arrangement of controls and inoculums 


\section{List of Tables}

\section{Chapter One}

Table 1.1 Plant materials used in Chinese papermaking in history ${ }^{[33]}$ 8

\section{Chapter Two}

Table 2.1 Resistance to fungal growth of Phormium fibres from 14 cultivars........22

Table 2.2 In vitro antifungal activity of amphotericin B and four standard coumarins, expressed as MIC $\left(\mu \mathrm{g} \cdot \mathrm{mL}^{-1}\right)$

Table 2.3 Antifungal activities of aqueous extracts of Phormium fibres from 14 cultivars of $P$. tenax

Table 2.4 Qualitative evaluation of antifungal activities of Phormium fibres from 14 cultivars of $P$. tenax.

\section{Chapter Three}

Table 3.1 Diagnostic daughter ions in ESI-MS/MS characterisation of aqueous extracts of Phormium fibres by reference to authentic compounds. Ions are those resulting from the loss of neutral molecules from $\mathrm{MH}^{+}$and/ or are characteristic fragments of the structure.

Table 3.2 ESI-MS/MS identification of selected products in aqueous extracts from fibres from six cultivars of $P$. tenax.

\section{Chapter Four}

Table 4.1 Diagnostic ions (m/z) used in ESI-MS/MS identification of photoproducts of UVA-irradiated aqueous 6,7-dihydroxycoumarin (esculetin) and 7-hydroxy-6-methoxycoumarin (scopoletin). Daughter ions result from the loss of neutral molecules from $\mathrm{MH}^{+}$.

\section{Chapter Five}

Table 5.1 List of Chinese handmade papers studied in this thesis 


\section{Chapter Six}

Table 6.1 Natural dyes used on Phormium textiles by Maori $[8,25,172]$ 132

Table 6.2 Determination of iron content of Phormium fibres from five cultivars 139

Table 6.3 ICP-MS determination of trace elements in ashed samples of Mahoe bark collected from three different regions in New Zealand 146

Table 6.4 $\mathrm{pH}$ values of Xuan paper before and after 4 days of accelerated aging $\left(100 \% \mathrm{RH}, 70^{\circ} \mathrm{C}\right)$

\section{Appendix A}

Table A1. Scheme for preparing dilution series of water-insoluble antifungal agents to be used in broth dilution susceptibility tests 173

Table A2. Numerical scoring of visual examination 174 


\section{Abbreviations}

$\begin{array}{ll}\text { CFU } & \text { colony-forming unit } \\ \text { CID } & \text { collision induced dissociation } \\ \text { DCM } & \text { dichloromethane } \\ \text { DDW } & \text { double distilled water } \\ \text { DMSO } & \text { dimethyl sulphoxide } \\ \text { EDS } & \text { energy-dispersive X-ray spectroscopy } \\ \text { EEMs } & \text { excitation-emission matrices } \\ \text { ESI-MS } & \text { electrospray ionisation mass spectrometry } \\ \text { FAAS } & \text { flame atomic absorption spectroscopy } \\ \text { FWAs } & \text { fluorescence whitening agents } \\ \text { GC } & \text { growth control } \\ \text { HOMO } & \text { highest occupied molecular orbitals } \\ \text { MCP } & \text { multi-channel plate } \\ \text { MIC } & \text { minimum inhibitory concentration } \\ \text { MID } & \text { mean integrated density } \\ \text { MP } & \text { megapixel } \\ \text { ICP-MS } & \text { inductively coupled plasma mass spectrometry } \\ \text { IMID } & \text { incremental mean integrated density } \\ \text { PDA } & \text { potato dextrose agar } \\ \text { Q } & \text { quadrupole } \\ \text { q } & \text { time-of-flight } \\ \text { RH } & \text { ultraviolet } \\ \text { ROS } & \text { relative humidity } \\ \text { SC } & \text { reactive oxygen species } \\ \text { SCE } & \text { sterile control } \\ \text { SEI } & \text { saturated calomel electrode } \\ \text { SEM } & \text { second electron image } \\ \text { SOD } & \text { scanning electron microscopy } \\ \text { TOF } & \text { superoxide dismutase } \\ \text { UV } & \text { XRF }\end{array}$




\title{
Chapter 1
}

\section{Introduction}

\author{
"A journey of a thousand miles begins with a single step." \\ - Chinese philosopher Laozi (604 - 531 BC)
}

\subsection{Motivation}

Two lignocellulosic fibrous materials of cultural and heritage value are studied in this $\mathrm{PhD}$ project. The first material of interest is the fibres from the leaves of Phormium tenax, the principal textile fibre used by Maori since pre-European times. The primary research goal was to understand the way it deteriorates, the factors that influence its stability, and the means to stabilise or preserve this material. An important motivation for this work was a previous study ${ }^{[1]}$ that had detected naturally-occurring coumarins in the fibre. These compounds can function as both fluorescent whitening agents and plant antifungal metabolites, and raised a plethora of questions to investigate:

- Do Phormium fibres have intrinsic resistance to fungal growth?

- What is the role of coumarins in the photodegradation of Phormium fibres? And what are the underlying mechanisms?

- Is there a relationship between fibre stability and the Phormium cultivar from which it is extracted? 
- What is the effect of mordant metal ions and environmental factors such as humidity on fibre deterioration?

These questions provided the initial momentum for this work and were reflected in the topics covered throughout the chapters of this thesis, justifying the broad encompassing title: "Stabilisation of Natural Colourants and Lignocellulosic Textiles". A variety of experimental techniques were employed to achieve this goal: mycological testing, mass spectral molecular identification, electron microscopic examination, elemental analysis and a board range of optical spectroscopies - absorbance, reflectance and fluorescence.

Another substantial part of this thesis deals with Chinese handmade papers, another important cultural material used in China. The main aim was to establish and compare the photostabilities of differently manufactured papers in order to provide fundamental reference information for conservation purposes.

\subsubsection{Outline}

A general background and overview of the two lignocellulosic materials studied is given in the introduction (Chapter 1). The main body of the thesis can be divided into two sections, dealing respectively with the biodeterioration (Chapters 2-3) and photodegradation (Chapters 4-6) of these materials.

Chapter 2 includes a series of antifungal assays on Phormium fibres of different cultivars. Chapter 3 is devoted to the mass spectral identification of naturallyoccurring coumarins and other antifungal agents in the fungal-resistant cultivars. The finding of a number of 7-hydroxycoumarins provides a linkage to the second section of the thesis, UV-induced photodegradation, with three chapters reporting (i) the photoyellowing of Phormium fibres and selected 7-hydroxycoumarins examined by spectroscopy and the production of reactive oxygen species (Chapter 4), (ii) similar photochemical and spectroscopic characterisations of Chinese handmade papers (Chapter 5), and (iii) the effect of mordant metal ions (Chapter 6). Chapter 6 also addresses the effects of mordant metal ions on the optical 
properties of Phormium fibres, both dyed and undyed, together with a high-spatial resolution elemental analysis of traditional Chinese handmade papers. The last and concluding part, Chapter 7, summarises the findings and insights gained through the work of the thesis. It also suggests possible future work to answer some of the questions arising from this project.

\subsection{Historical Background of Materials}

A brief history of the materials studied in this work, Phormium fibres and traditional Chinese handmade papers, covering their provenance, manufacture, characteristics and some previous research is given below.

\subsubsection{Phormium Fibres}

Phormium tenax, an indigenous plant to New Zealand and Norfolk Island, provided the main weaving material traditionally used by Maori and has played an important role in the culture, history and economy of New Zealand.

\section{Traditional Uses and Processing}

The genus Phormium includes P. tenax (harakeke) and P. cookianum (wharariki), and both are commonly known as New Zealand flax as the morphology of the leaf fibre is similar to that of linen (Linum usitatissimum) although there is no botanical relationship. The early Maori settlers in New Zealand discovered how to weave the fibres from the leaves of Phormium plants, particularly P. tenax, and adopted it as a substitute for paper mulberry (Broussonetia papyrifera), which was used to produce bark cloth in their ancestral Polynesian homeland but which does not grow in New Zealand's cooler climate ${ }^{[2]}$. P. cookianum was not widely used due to its long, drooping leaves and coarse, brittle fibres ${ }^{[3]}$. Before contact with Europeans, the long and strong leaf fibres (muka) of $P$. tenax were used by Maori 
to weave clothing and make cordage, while the strips of the leaf (whenu) were used for plaiting mats and domestic utensils ${ }^{[4]}$.

Today, such items are still produced by artists and small groups such as Te Roopu Raranga Whatu o Aotearoa, the Maori Weavers' Group, to meet present cultural needs and as commercial products (e.g. for tourism). P. tenax has become an icon of contemporary New Zealand culture with the status of treasure (taonga) under Article II of the Treaty of Waitangi and Ngai Tahu Claims Settlement Act $1998^{[5]}$.

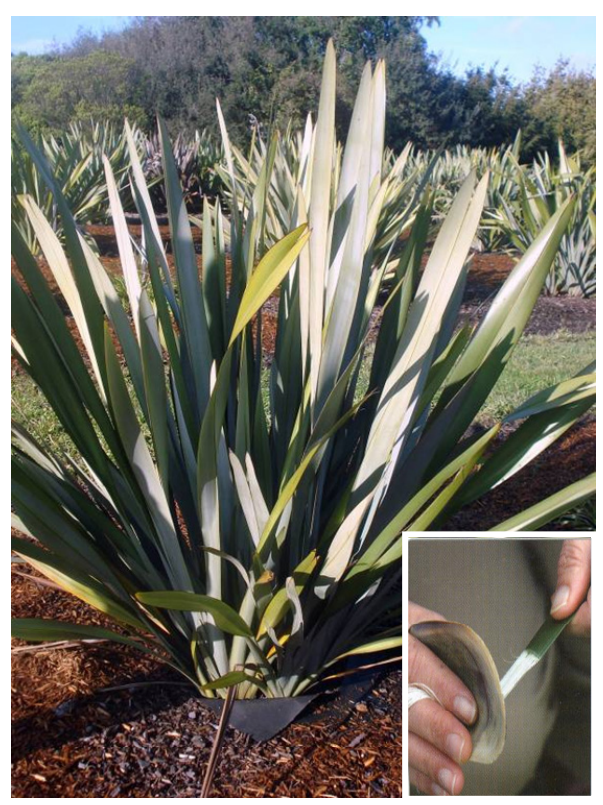

Figure 1.1 Plantation of $P$. tenax and the traditional fibre processing method (stripping with a mussel shell).

Figure 1.1 shows the processing of Phormium fibres in a traditional Maori practice. Leaves are collected from plants of one year to 18 months old. The green epidermal layers of each leaf are mechanically scrapped off using the sharp edge of a mussel (bivalve mollusc) shell. The inner long strands of fibres are extracted by one of the two traditional methods (haro and takiri), or a combination of both. The whole process is very labour-intensive. Details for fibre extraction, preparation and weaving techniques are well documented by $\mathrm{Mead}^{[6]}$ and Te 
Kanawa $^{[7]}$. Further processing including washing, rolling, beating and dyeing may occur before weaving.

The yield and quality of Phormium fibre varies significantly from plant to plant, as do the leaves in their form and colour ${ }^{[8]}$. Maori distinguished a number of varieties/cultivars of $P$. tenax based on qualities such as strength, softness, durability, colour, ease of extraction and quantity of fibre ${ }^{[9]}$. Some desirable cultivars were selected and kept in cultivation according to their suitability for different weaving and plaiting purposes ${ }^{[2,8,9]}$. An example is the Rene Orchiston Collection of sixty of the most favoured cultivars gathered by Mrs Rene Orchiston in the 1950s with detailed records of plant provenance, names and uses. The collection is now part of the New Zealand National Flax Collection kept at Landcare Research Crown Research Institute in the South Island of New Zealand and is providing resource materials for contemporary weavers and researchers ${ }^{[9]}$.

\section{The Phormium-Fibre Industry}

From the time of the first contacts, Europeans recognised the potential of Phormium fibres for the manufacture of marine rope and sacking ${ }^{[10]}$. Since early in the $1800 \mathrm{~s}$, a trade in hand-stripped fibres, initially purchased from Maori, was established with exports from New Zealand to Australia and England ${ }^{[9,11]}$. In the 1860 s, a stripping machine to remove the epidermal layer was developed leading to a considerable expansion of the commercial Phormium-fibre industry, although the machine-processed fibres were considered inferior in colour and quality ${ }^{[9,10]}$. For a time Phormium fibre was New Zealand's biggest export, and constituted up to $20 \%$ of the total income in the early years of the $20^{\text {th }}$ century. Early attempts were made to mechanically spin yarn and weave fabric ${ }^{[3]}$. However, the industrial production declined after the first World War because of unstable prices, diseased plants, and competition from synthetic fibres ${ }^{[9]}$. The last Phormium-fibre mill, located at Foxton in the North Island of New Zealand, closed in the 1980s ${ }^{[11]}$. 


\section{Scientific Research}

The first scientific description of Phormium fibre was made by French naturalist Labillardière in 1803, who visited the far north of the North Island in 1793 and cultivated the plant in France ${ }^{[12]}$. In the $19^{\text {th }}$ and early $20^{\text {th }}$ centuries, the commercial fibre industry was interested in Phormium selection and breeding as a means to improve the quantity and quality of fibre products ${ }^{[11]}$. In early 1910 , Cross provided a detailed characterisation of Phormium plants and fibres from different cultivars ${ }^{[13,14]}$. Machinery processing of Phormium fibre and very recently the production of blends with polypropylene, cotton, and cotton/polyester have been studied ${ }^{[3]}$.

Since the closing of the commercial fibre industry there has been a resurgence of interest in the traditional/cultural uses of Phormium plants $^{[11]}$. Recent studies have focused on the selection of weaving cultivars in order to gain insight into the relationship between fibre characteristics and weaving and/or plaiting suitability. The physical and morphological properties (e.g. length, linear density, tenacity and extension) of leaves ${ }^{[15,16]}$, fibres ${ }^{[3-5,11,17]}$ and strips ${ }^{[18]}$ of different weaving cultivars have been examined. Although largely qualitative, the fibre characteristics differed markedly among Phormium cultivars and were found to suit their traditional uses. Genetic introgression between $P$. cookianum and $P$. tenax, and the stabilisation of hybrid derivatives in the long history of selection were likely to contribute to the cultivar differences ${ }^{[19,20]}$. Environmental effects ${ }^{[4,}$ ${ }^{21]}$ and the part of leaf from which the fibre was extracted ${ }^{[3,11]}$ were also shown to be important.

Some research was undertaken to understand the deterioration mechanisms of Phormium fibre in order to assist in the conservation of Maori textile artifacts in museums. For example, in a comparative study, the traditional weaving cultivars were found to contain lower levels of guaiacyl lignin, known to have poor photostability, than other cultivars ${ }^{[22]}$. Smith detected naturally-occurring coumarins in undyed Phormium fibres (cultivar not given), the presence of which was considered to contribute to both the whiteness and photoyellowing of the 
fibre $^{[1]}$. The effect of a black iron-tannin dye on the decorated fibres/textiles was also investigated ${ }^{[23-25]}$.

Other studies were aimed at exploiting the antimicrobial properties of extracts from Phormium plants that have been used in the traditional Maori medicine (rongoa). For example, Phormium fibres were traditionally used as bandages and wound dressings ${ }^{[26]}$. Compounds with antifungal and antiseptic activities were isolated from the plant leaves, seeds and rhizomes ${ }^{[27-29]}$.

\subsubsection{Chinese Handmade Paper}

Manufactured from indigenous plants in China, Chinese handmade papers have been regarded as an important cultural heritage material in China. Various papers were developed and produced by traditional papermaking methods since antiquity and they are still widely used today for calligraphy, painting and printing.

\section{History}

Paper originated in China. Although Tsai Lun is generally credited with the invention of paper using the pulp of old rags, hemp, tree bark and fishing net in $105 \mathrm{AD}$, there are archeological paper fragments excavated that date back to the $2^{\text {nd }}$ century $B C$, indicating that Tsai may have just improved an earlier process. By the end of the $5^{\text {th }}$ century, paper was widely used for writing and in daily life in China $^{[30]}$. From China, papermaking slowly spread to the rest of the world and the technology has undergone changes in the movement from Eastern to Western civilisations. For example, Oriental papers were characterised by their soft, thin and translucent properties suitable for writing on only one side with a brush, whereas Western papers were sized with animal glue or gelatin to give a thicker and stronger surface in order to allow the use of an abrasive quill pen as well as to withstand the acidic iron-gall ink $^{[31,32]}$. 


\section{Source Plant Materials}

According to historical records, a broad range of indigenous plants have been used as raw materials for papermaking in China $^{[33]}$. As shown in Table 1.1, the plant materials used for papermaking varied chronologically and regionally, and can be categorised into three groups: bast, tree bark and grasses ${ }^{[33]}$. The bast-producing plants of hemp, jute, flax and ramie (all referred to as $m a$ in ancient Chinese documents) were probably the earliest materials used for papermaking, followed by paper mulberry, rattan, bamboo, straw and blue sandalwood. However, the use of $m a$ papers declined after the Tang dynasty (618 - 907 AD), and the supply of rattan was exhausted by the early Song (960 - 1279 AD). Most of the other materials have continued in use to the present ${ }^{[33]}$.

Table 1.1 Plant materials used in Chinese papermaking in history ${ }^{[33]}$

\begin{tabular}{|c|c|c|c|c|}
\hline Fibre & Plant & Scientific Name & First Use (Dynasty) & $\begin{array}{c}\text { Region of } \\
\text { Origin }\end{array}$ \\
\hline \multirow[t]{5}{*}{ Bast } & Hemp & Cannabis sativa & Western Han (206 BC - 9 AD) & \\
\hline & Jute & Corchorus capsilaris & Western Han (206 BC - 9 AD) & \\
\hline & Flax & Linum perenne & Western Han (206 BC - 9 AD) & \\
\hline & Ramie & Boehmeria nivea & Western Han (206 BC - 9 AD) & \\
\hline & Rattan & Calmus rontang & $\begin{array}{l}\text { Jin }(265-420 A D) \text { to early } \\
\text { Song }(960-1279 A D)\end{array}$ & $\begin{array}{l}\text { Southeastern } \\
\text { China (extinct) }\end{array}$ \\
\hline \multirow[t]{3}{*}{ Bark } & $\begin{array}{l}\text { Paper } \\
\text { mulberry }\end{array}$ & $\begin{array}{c}\text { Broussonetia } \\
\text { papyrifera }\end{array}$ & Western Han (206 BC - 9 AD) & \\
\hline & Mulberry & Morus alba & Tang $(618-907 A D)$ & Northern China \\
\hline & $\begin{array}{c}\text { Blue } \\
\text { sandalwood }\end{array}$ & $\begin{array}{c}\text { Pteroceltis } \\
\text { tatarinowii Maxim. }\end{array}$ & Tang $(618-907 \mathrm{AD})$ & Anhui \\
\hline \multirow[t]{2}{*}{ Grass } & Bamboo & Phyllostachys aurea & Tang $(618-907$ AD) & $\begin{array}{l}\text { Southeastern } \\
\text { China }\end{array}$ \\
\hline & Rice straw & Oryza sativa & Before Song (960 - 1279 AD) & \\
\hline
\end{tabular}

Mixing fibres from different plants was common practice in Chinese papermaking. Early in the Tang dynasty, there were papers made from hemp mixed with paper mulberry. With the decline of hemp paper from the Song dynasty, mixtures of bast with bamboo, bast with straw, and bamboo with straw dominated the Chinese 
paper market ${ }^{[34]}$. The selection of fibres and the proportions of raw materials have been suggested to have had a decisive role in the quality and special features of different papers ${ }^{[33]}$. For example, the bark fibre of blue sandalwood is believed to provide the famous Xuan paper with characteristic ink-absorbing quality and finish that is favoured for Chinese calligraphy and painting, while the proportion of rice straw present in the pulp determines the durability and softness of paper.

\section{Traditional Chinese Papermaking}

The essence of papermaking is the reduction of raw plant materials into their component individual fibres and the reassembly of fibres into an intermeshed thin sheet of solid material ${ }^{[35]}$. Needham and Tsien devoted half a volume in their multi-volumed work, "Science and Civilisation in China", to the history and manufacture of traditional Chinese paper ${ }^{[33]}$. The methods of traditional papermaking in China varied slightly according to materials, periods and regions, but the basic processes have remained more or less the same throughout the centuries. The general procedures for Chinese papermaking include fibre preparation (with repeated soaking, cooking, rinsing, and sun bleaching of the raw plant materials), pounding, pulping, sheet formation, pressing and drying ${ }^{[33,34,36]}$. Some special features of the methods used are given below:

- Initially, raw plant materials are macerated by repeated soaking in alkaline solution (with lime or wood ash), beating, "cooking" (steaming or boiling) and rinsing (followed by repeated soaking and cooking) until the bark and husk are completely sloughed off and only the fibres remain (Figure 1.2A). "Cooked" fibres are bleached by exposure to sunlight lying flat and spread over an area of several acres until they become white and soft (Figure 1.2B). This first stage of fibre preparation, also known as "removing the green", is an arduous and painstaking process, and may take more than one hundred days. 
- The refined fibres are pounded by a hydraulic pestle into pulps (Figure 1.2C). They are further cleaned by placing into a fine-mesh cloth bag and rinsing in running water (Figure 1.2D). Then the fibres are poured into the pulp vat where starch paste or mucilaginous gums from the leaves of some deciduous trees, such as Hibiscus manihot and Actinidia chinensis (kiwifruit), are added as sizing agents to improve the evenness of fibre distribution and inter-fibre binding strength ${ }^{[33]}$.

- Sheets of paper are formed by casting the fibres in suspension on a screen mould (usually made of a mat woven of bamboo strips). The thickness of the paper depends on the way in which the screen is manipulated. After about a hundred sheets are stacked, they are pressed together to remove the water (Figure 1.2E).

- A double wall of earthen bricks is employed in the drying process. A fire is lit in the hollow centre of the walls, so heat can spread to the wall surface when the bricks become hot. The wet sheets of paper are placed onto the wall one by one, baked to dryness, and taken off as finished sheets (Figure 1.2F).

From the $20^{\text {th }}$ century, the traditional method of manufacture of Chinese paper has been modified by Western papermaking technology. For example, sodium hydroxide and chlorine water have been used as bleaching agents in the preparation of bast-fibre pulps; and specially designed machinery has been introduced for the beating and pounding processes ${ }^{[36]}$. However, papers produced using these modern techniques are generally considered inferior in quality to papers produced by traditional manufacturing methods. 

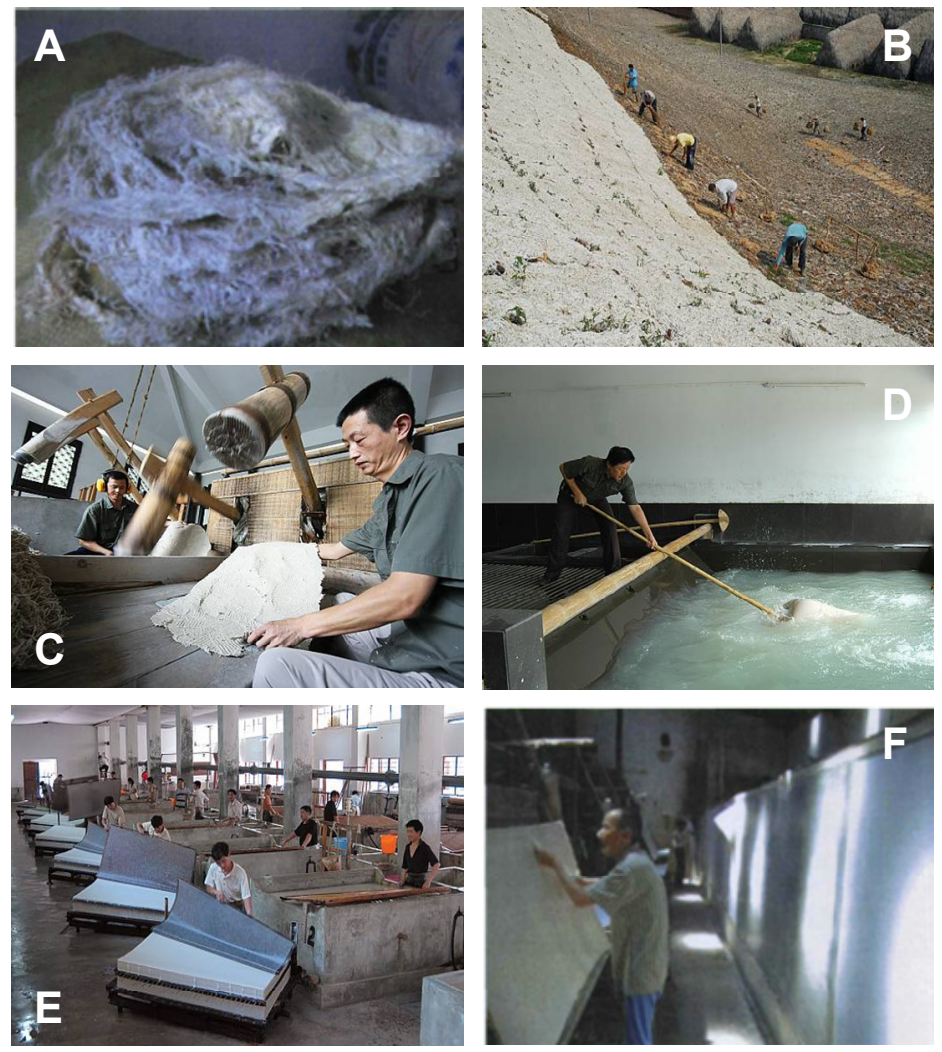

Figure 1.2 Methods of traditional Chinese papermaking ${ }^{[36,37]}$ : macerated plant fibre (A), sunning of fibre (B), pounding with wooden pestles (C), purifying pulps in running water $(D)$, sheet formation $(E)$ and drying on a heated wall $(F)$. Pictures $B$ to $E$ were taken by Feisong Huang at the Red-Star Xuan papermill in Jing County, Anhui province, China. 


\section{Scientific Research}

Most research on Chinese handmade papers have been isolated studies on paper handcrafts and techniques used in different regions ${ }^{[36,38]}$. Scientific studies are comparatively rare and mainly focus on the identification of fibres, dyes and inks from archaeological paper samples to place them in a historical context and also for the authentication of documents ${ }^{[39-43]}$.

In 1997, Tsai and Reyden's report on the conservation of a Chinese woodblock print was an important contribution to the conservation literature published in English $^{[34]}$. In order to find suitable and compatible repair materials for the damaged woodblock print, the authors compared a number of modern Chinse papers using a series of analytical techniques including SEM-EDS, colorimetry, tensile strength, $\mathrm{pH}$ measurements and artificial ageing ${ }^{[34]}$. The presence of characteristic phytoliths, plant microscopic bodies, observed in the rice straw fibre present in Xuan paper has led to a novel approach, phytolith analysis, for the identification of the source of plant materials in ancient papermaking ${ }^{[34,44]}$.

In recent years, there has been widespread investigation into the permanence and durability of modern Chinese handmade papers during artificial ageing. The application of alum ${ }^{[45]}$, bleaching agents ${ }^{[46]}$, sizing ${ }^{[47]}$, colourants ${ }^{[48]}$ and coatings ${ }^{[49]}$ in the manufacture or conservation treatments and their effects on the performance and optical properties of various papers have been evaluated in these research activities. Other studies are directed towards improving the storage environment of paper artefacts. Microwave sterilisation ${ }^{[50]}$ and some atmosphere and humidity controlling agents (e.g. $\mathrm{O}_{2}$ absorbers and silica gels) ${ }^{[51]}$ have been developed to reduce foxing (see page 109) and biodeterioration of paper materials. 


\section{Chapter 2}

\section{Mycological Screening of Phormium Fibres}

Phormium has traditionally been used in Maori medicine for its antifungal properties. However, the extent to which the activity and extractability of the antifungal agents (coumarins and other compounds) in the leave fibres is unknown. This chapter covers an examination of the susceptibility to fungal growth of Phormium fibres of 14 selected cultivars and evaluations of the antifungal activities of aqueous extracts compared with a number of standard coumarins.

\subsection{Introduction}

Biodeterioration is an issue of major concern in the stabilisation, preservation and conservation of cellulosic textiles. Microbial spoilages on textiles from fungi, bacteria (especially actinomycetes) can cause loss of strength and elongation, discolouration, staining and associated unpleasant odours ${ }^{[52]}$. These microbiological agents can be of cellulolytic or noncellulolytic species. The cellulolytic strains damage the chemical structure of lignocellulosic fibres, while the noncellulolytic attack substances added in manufacturing, such as finishes, adhesives, colourants and fillers ${ }^{[52]}$.

The most severe indoor forms of biodeterioration are primarily caused by cellulolytic fungi with broader environmental tolerance than normal bacteria and actinomycetes $^{[53]}$. Certain xerophilic (capable of growing at reduced water activity) 
species of Aspergillus and Penicillium are particularly harmful as they can live with water content as low as $62-65 \% \mathrm{RH}$, which is common in museums and libraries ${ }^{[53]}$. The major theories about the mechanisms of cellulolytic attack, choice of potential biocides and environmental conditions have been extensively reviewed $^{[53,54]}$. Degradation of textiles exposed outdoors (e.g. archaeological textiles buried in soil) has been suggested to result from bacteria associated with certain species of myxomycetes and actinomycetes ${ }^{[55]}$. However, the toxic fungicides often used to treat this biodeterioration of textiles have deleterious effects on environment and are toxic to humans ${ }^{[56]}$.

A number of higher plants have the ability to defend themselves against fungal attack by in vivo synthesis of antifungal chemicals that may be constitutive or stress-induced $^{[57,58]}$. Besides its role as their principal fibre plant, P. tenax also played an important role in Maori's traditional medicine. For example, the flax gum had been found to have antiseptic, anaesthetic and blood clotting properties and was topically applied for skin-care ${ }^{[26]}$. Compounds with antifungal effects have been isolated from the roots, seeds and rhizomes of the plant, such as 7hydroxy-5-methoxy-6-methylphthalide and musizin ${ }^{[27-29]}$. However, the antifungal properties of the leave fibres have not been investigated. Recently, fluorescence that is consistent with a number of naturally-occurring coumarins, which function as fluorescent whitening agents, was detected in the leaf fibres of Phormium in our laboratory $^{[1]}$; and this finding has prompted the present study.

Many coumarins are well known for their multiple biological activities including antifungal properties. Examples are coumarin, 7-hydroxycoumarin (umbelliferone), 7-methoxycoumarin (herniarin), 7-hydroxy-6-methoxycoumarin (scopoletin) and 6,7-dihydroxycoumarin (esculetin), which all have a wide distribution in higher plants ${ }^{[57,59-61]}$. Coumarins are constitutively expressed in many plants, and, together with other antifungal metabolites, their accumulation can be induced as a phytoalexin in response to fungal attack, mechanical tissue damage or treating with elicitors ${ }^{[57,62,63]}$.

The leaves and fibres from Phormium have been used since pre-European times 
for the production of cordage, mats, containers, cloaks and many items of clothing $^{[4]}$. An understanding of any antifungal activity present in the fibres may assist in the preventive conservation and restoration of museum objects. There is also commercial potential for the use of Phormium fibres for novel textiles or textile-blends that reduce or eliminate the need for environmentally undesirable chemical antifungal treatments of textiles.

For the first time, the study of Phormium fibres is extended to fungal deterioration based on the finding of naturally-occurring coumarins and the observation of natural variations in different cultivars upon fungal attack ${ }^{[64]}$. In this chapter, 14 genetically distinct Phormium fibres were tested in order to investigate whether resistance to fungal activity varied across the genus, or whether a particular cultivar could be chosen for novel fibre uses because of its resistance.

\subsection{Fungal Susceptibility Test of Solid Fibres}

\subsubsection{Materials and Methods}

\section{Fibres}

The Phormium fibres studied were of 14 traditional cultivars of P. tenax (listed in Table 2.3) from the National New Zealand Flax collection ${ }^{[9]}$ maintained by Landcare Research (Lincoln, New Zealand). These cultivars, whose genetics had been preserved for many years, were chosen as representatives of a range of morphological characteristics and fibre qualities. Fibres were extracted from leaves of $P$. tenax using the traditional Maori method (more information about this is given in Chapter 1). All fibre samples were packaged in paper bags and stored in the dark prior to use. 


\section{Microorganisms and Inoculum Quantitation}

Two common filamentous fungal strains Penicillium glabrum and Aspergillus wentii were supplied by the International Collection of Microorganisms from Plants (ICMP) maintained by Landcare Research (Auckland, New Zealand). $P$. glabrum (ICMP 1199) was originally obtained from stored timbers and A. wentii (ICMP 589) from a straw basket. The fungi were maintained long-term on carrot root extract agar and sub-cultured monthly for inoculum preparation on potato dextrose agar (PDA, Roth) at $25^{\circ} \mathrm{C}$.

Conidial suspensions were prepared by covering 7-day-old mature colonies with 2 $\mathrm{mL}$ of sterile saline $(0.9 \%)$. Approximately $1.5 \mathrm{~mL}$ of the mixture of conidia and mycelial fragments were harvested and pipetted into a sterile Eppendorf tube. For A. wentii, a drop of Tween-20 (Polyoxyethylene sorbitan monolaureate, Gerbu) was added to facilitate the dispersion and suspension process ${ }^{[65]}$. Large particles were allowed to settle. The upper supernatant was diluted with $0.9 \%$ saline to approximately $5 \times 10^{5}$ colony-forming units (CFUs) per mL. A count of CFUs per $\mathrm{mL}$ was obtained by dilution and plating onto PDA and counting after incubation at $25^{\circ} \mathrm{C}$ for $48 \mathrm{hrs}$.

\section{Inoculation and Incubation}

Portions of fibres weighing $0.1 \mathrm{~g}$ were cut into short lengths $\sim 3-4 \mathrm{~mm}$ long and compressed into self-adhering monolithic discs of $13 \mathrm{~mm}$ diameter using a hydraulic press. After 15 min of autoclave sterilisation at $121{ }^{\circ} \mathrm{C}$, individual discs were placed in the wells of 24-well tissue culture plates. Discs were inoculated with 2,000 CFUs contained in $400 \mu \mathrm{L}$ of sterile double distilled water (DDW). Control discs were treated with sterile water only. The discs in the tissue culture plates were incubated for 7 days at $25{ }^{\circ} \mathrm{C}$ inside a sealed plastic container that had enough sterile water in its base to prevent the discs from drying out. All operations were conducted inside a vertical clean-bench (Labconco, U.S.) to avoid external, adventitious microbial contamination. 


\section{Visual and Digital Evaluation of Fungal Growth}

After 7 days of incubation, the inoculated discs or inoculums turned darker in appearance and were discoloured by the colonising fungi (green for P. glabrum, pale yellow for $A$. wentii) compared with the controls. In vivo observations by confocal microscopy (TCS 4D, Leica Lasertechnik, Germany) indicated that the darkening of the inoculated discs resulted from the germination of fungal mycelia and the degradation of the fibres (Figure 2.1).
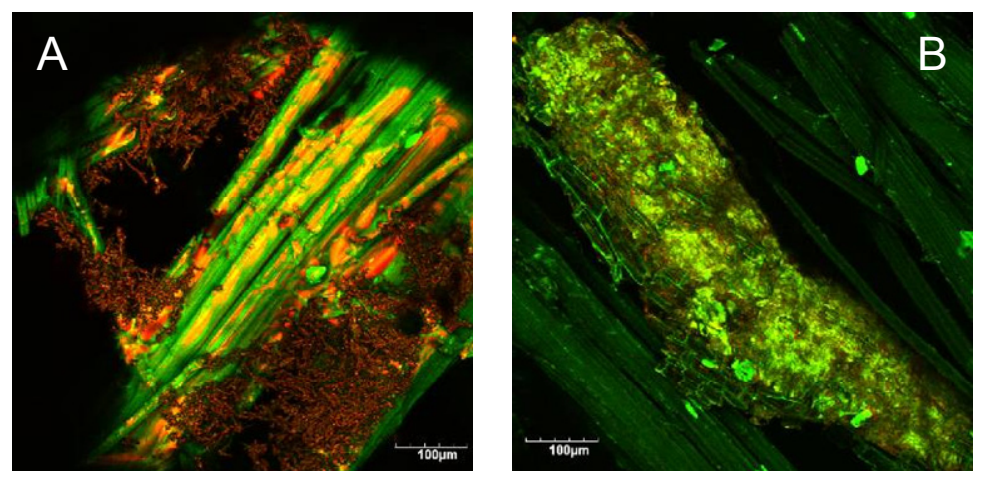

Figure 2.1 Confocal images of Phormium fibres (green auto-fluorescence at 488 $\mathrm{nm}$ ) inoculated by $P$. glabrum (red auto-fluorescence at $647 \mathrm{~nm}$ ) after 7 days of incubation. Yellow areas indicate the overlapping of the red and green signals. $B$ is an enlarge view of a degraded fibre in contact with fungal mycelia.

Digital images of inoculated discs loaded in 24-well culture plates (Figure 2.2A) were taken by a four megapixel (MP) camera (Canon PowerShot G3) mounted on a MP4 stand (Polaroid) with oblique lighting from two light panels (MEDALight LP-400). The evaluation of fungal growth can therefore be made both visually and digitally as indicated by the colour change of inoculated discs. Visual observations with unaided eyes or a microscope of the discs shown in Figure 2.2A for example, gives a ranking of the fungal-induced darkening of different cultivars: Awahou $\approx$ Whareongaonga $>$ Potaka $>$ Tapamangu. 
A.

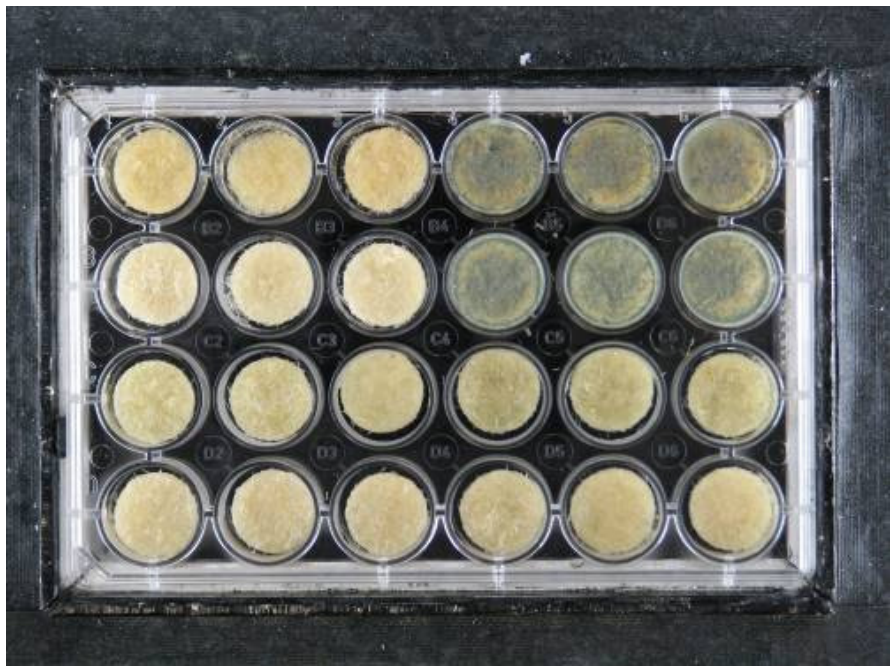

B.

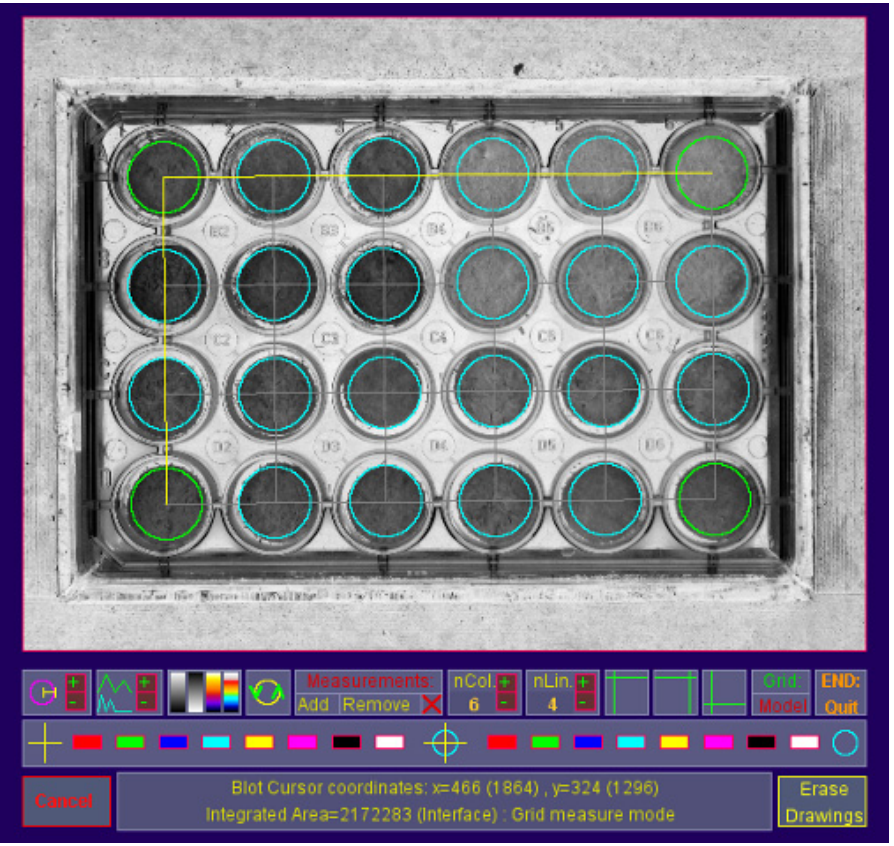

Figure 2.2 Fungal susceptibility test of fibres from four Phormium cultivars (rows from top to bottom are: Awahou, Whareongaonga, Potaka and Tapamangu). Columns 1 to 3 (left-hand side) are controls; columns 4 to 6 (right-hand side) are inoculums with $P$. glabrum $\left(400 \mu \mathrm{L} \times 500 \mathrm{CFUs} \cdot \mathrm{mL}^{-1}\right)$. A is the original image. $B$ is the interactive graphic interface of Dot Blot Analyzer for ImageJ after 8-bit conversion. 
In the digital method, the captured images were analysed using Gilles Carpentier's "Dot Blot Analyzer for ImageJ", which is a public domain image processing software developed at the U.S. National Institutes of Health ${ }^{[66,67]}$. As shown in Figure 2.2B, a circular area covering at least $90 \%$ of each disc was selected and the sum of the pixels inside the selection, which ImageJ terms the "integrated density", as a measure of the extent of darkening was determined. The circular area of same size was selected for all discs on a plate.

After determining the grey-level values for the discs (in triplicate), the mean integrated density (MID) was calculated out; and the difference between controls and inoculums (incremental MID or IMID) for each cultivar was used to give a quantitative evaluation of its fungal-induced colour change. For example, the IMID calculated was: Whareongaonga $(1.9 \mathrm{MP})>$ Awahou $(1.6 \mathrm{MP})>$ Potaka $(0.47 \mathrm{MP})>$ Tapamangu $(0.25 \mathrm{MP})$, which gave a similar but more precise ranking. The ImageJ method was therefore utilised in the later part of the project as a more objective evaluation of fungal growth.

\subsubsection{Results and Discussion}

Figures 2.3 shows the MIDs, with error bars $(2 \times$ standard deviations $)$, of controls and inoculums for all 14 Phormium cultivars tested against $P$. glabrum and for a subset of 7 cultivars (chosen randomly) challenged with $A$. wentii. The results can be summarised as below:

- Control discs had slight but not statistically significant natural variations in colour which may be due to the genetic variations of Phormium fibres.

- Inoculums were generally darker than controls as a result of fungal growth.

- Considerable intercultivar variations were observed in the difference between inoculums and controls as IMID shown in Figure 2.4. 

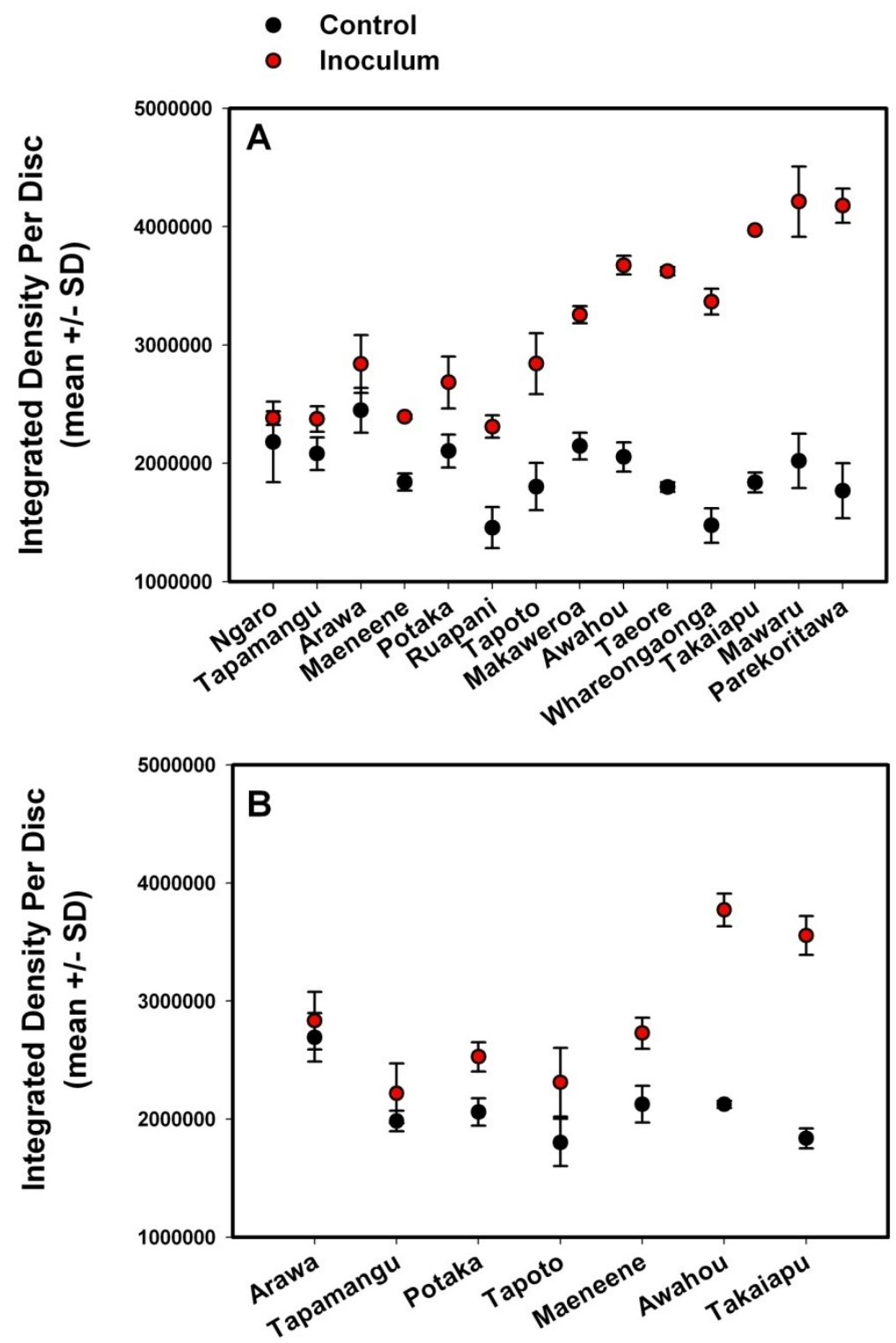

Cultivars of $P$. tenax

Figure 2.3 Colour variations of Phormium fibres on inoculation of $P$. glabrum (A) and $A$. wentii (B), inoculum: 2,000 CFUs per $0.1 \mathrm{~g}$ of fibre, 7 days of incubation 


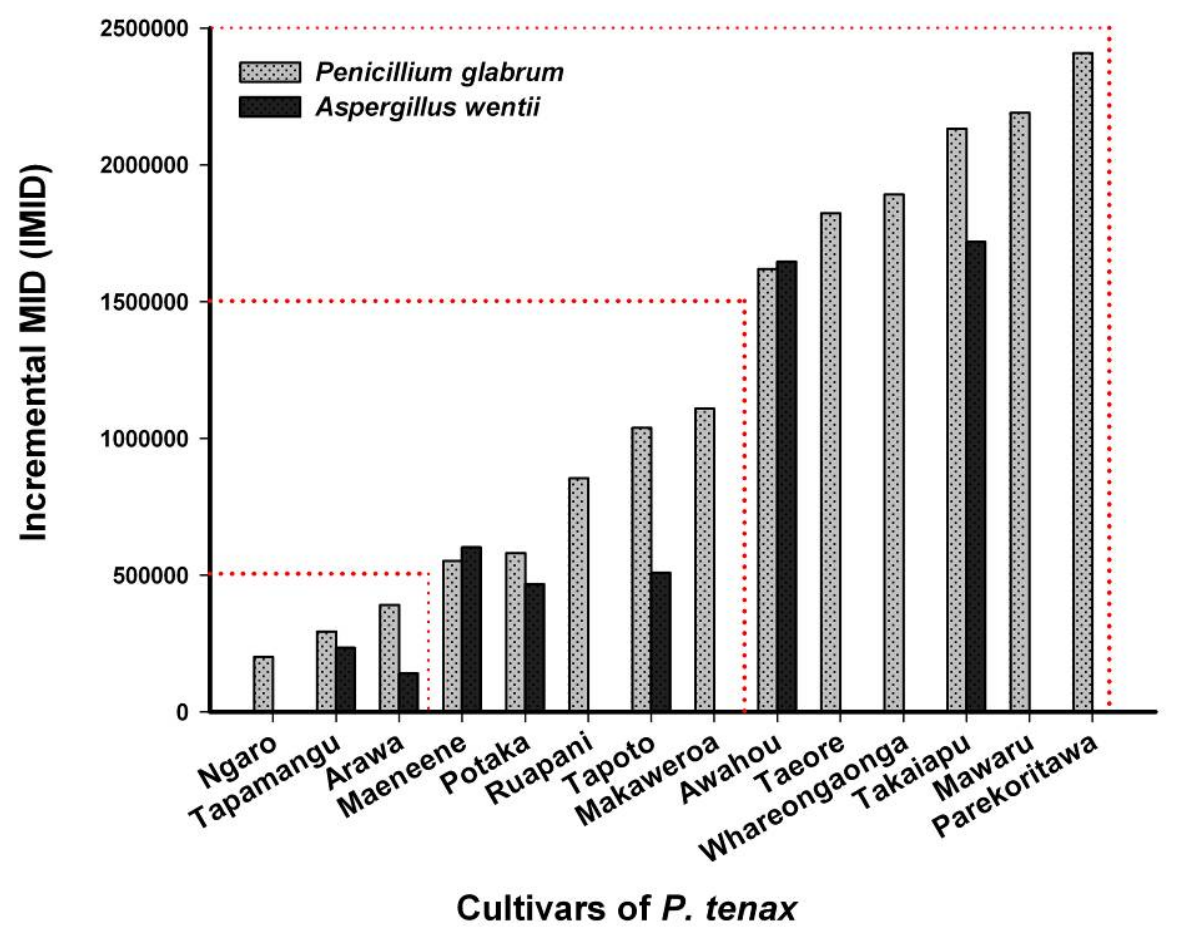

Figure 2.4 Fungal-induced colour change between controls and inoculums (incremental MID or IMID) of Phormium fibres from 14 cultivars

Thus, the tested fibres from various Phormium cultivars differ in their ability to resist fungal growth in the moist environment $\left(75 \% \mathrm{RH}, 25{ }^{\circ} \mathrm{C}\right)$ in which they were tested. An arbitrary classification of the fibres can be made in terms of cultivars having low, moderate or high resistance to Penicillium and Aspergillus growth (Table 2.1). The classification was based on dividing the range of IMID units into three approximately equal segments (Figure 2.4). The choice of fungal species made subtle difference to the variations in fungal-induced colour change observed in different cultivars. The 7 cultivars tested against $A$. wentii were classified as having similar response against $P$. glabrum, however the limit for high resistance to the growth of $A$. wentii was slightly lowered as the mycelia were less coloured than P. glabrum. 
Table 2.1 Resistance to fungal growth of Phormium fibres from 14 cultivars

\begin{tabular}{c|c|c}
\hline \multirow{2}{*}{$\begin{array}{c}\text { Fungal* } \\
\text { Resistance }\end{array}$} & \multicolumn{2}{|c}{ Cultivars of $\boldsymbol{P}$. tenax } \\
\cline { 2 - 3 } Low & $\begin{array}{c}\text { Awahou; Taeore; Whareongaonga; } \\
\text { Takaiapu; Mawaru; Parekoritawa }\end{array}$ & Awahou; Takaiapu \\
\hline Moderate & Maeneene; Potaka; Ruapani; Tapoto; \\
Makaweroa & $\begin{array}{c}\text { Maeneene; Potaka; } \\
\text { Tapoto }\end{array}$ \\
\hline High & Ngaro; Tapumangu; Arawa & Arawa; Tapamungu; \\
\hline * Low = IMID > 1,500,000; Moderate = IMID between 500,000 to $1,500,000 ;$ High = IMID < \\
500,000 (P. glabrum) or IMID <300,000 (A. wentii)
\end{tabular}

There are several possible causes of the observed cultivar variations in resistance to fungal growth, such as:

- Differing amounts, types or availability of water-soluble, antifungal agents present in the fibres;

- Different fungal nutrients or nutrient availability in the fibres as a result of different chemical constituents or fibre architecture;

- Differing quantities of antifungal hydrolytic products formed in the autoclaving process.

In summary, it has been shown that fibres extracted from different $P$. tenax cultivars differ in their ability to resist fungal growth which is an important cause of biodeterioration of cultural artifacts made from this plant material. The pattern of fungal growth susceptibilities between the tested cultivars was similar for the two fungal species. However, it is not clear from this research whether this is due to similarities in the amounts or availability of antifungal substances or of nutrients in the studied Phormium fibres. 


\subsection{Antifungal Assay of Aqueous Extracts}

To separate the effects of nutrient status from the intrinsic antifungal activity of Phormium fibres, a standard in vitro antifungal susceptibility assay of aqueous extracts of the solid fibres was employed with some modifications. In this section, the antifungal activities of one established agent (amphoterocin B), four authentic coumarins and the aqueous extracts of fibres from 14 cultivars of $P$. tenax were evaluated. The fungal species studied were P. glabrum and A. wentii as for the previous assay of fungal growth on the solid fibres.

\subsubsection{Materials and Methods}

\section{Extraction}

Fibres from 14 P. tenax cultivars were extracted in double distilled water $(0.1 \mathrm{~g}$ of fibre per $1 \mathrm{~mL}$ of DDW) at room temperature overnight assisted with a $30 \mathrm{~min}$ initial sonication of the samples. These aqueous extracts were then filter-sterilised (Rotilabo-Spritzenfilter, $0.22 \mathrm{~mm}$, Roth) and used in the biochemical antifungal activity assay described below.

Another extraction was performed on the cultivars Arawa, Ngaro and Tapamangu (cultivars that have been shown to have high resistance to fungal growth in the previous fibre test), by soaking $50 \mathrm{~g}$ of finely chopped fibres in $300 \mathrm{~mL}$ of double distilled water (DDW) overnight after an initial $30 \mathrm{~min}$ of sonication. The suspension of fibres was then filtered out and the filtrate (about $250 \mathrm{~mL}$ ) was extracted with half volume of dichloromethane (DCM). The separated DCM layer was evaporated to dryness under reduced pressure in a rotary evaporator (Rotavapor ${ }^{\circledR}$ R-210, BÜCHI). Residues were redissolved in dimethyl sulphoxide (DMSO) to make a solution with a final concentration of approximately $100 \mathrm{~g} \cdot \mathrm{L}^{-1}$. Solutions were stored at $4{ }^{\circ} \mathrm{C}$ prior to testing. 


\section{Coumarins}

In addition to the aqueous/DCM fibre extracts, antifungal assays were carried out on standard coumarin and three hydroxylated derivatives in DMSO. Stock solutions of coumarin (98\%, Sigma-Aldrich), 7-hydroxycoumarin (umbelliferone, $99 \%$, Chem Service), 6,7-dihydroxycoumarin (esculetin, $98 \%$, Sigma-Aldrich) and 7-hydroxy-6-methoxycoumarin (scopoletin, $98 \%$, Sigma-Aldrich) were prepared and filter-sterilised.<smiles>C=C1C=Cc2ccccc2O1</smiles>

Coumarin<smiles>O=c1ccc2cc(O)c(O)cc2o1</smiles>

Esculetin (6,7-dihydroxycoumarin)<smiles>O=c1ccc2ccc(O)cc2o1</smiles>

Umbelliferone (7-hydroxycoumarin)<smiles>COc1cc2ccc(=O)oc2cc1O</smiles>

(7-hydroxy-6-methoxycoumarin)

Figure 2.5 Structures of coumarins used in this study

\section{Colorimetric Antifungal Assay}

The in vitro antifungal activities of extracts and standard coumarins were assessed using a standardised broth macrodilution testing for filamentous fungi described in the U.S National Committee for Clinical Laboratory Standards (NCCLS, reference document M38-A) ${ }^{[65]}$ with modifications (as given below).

Inoculum suspensions were RPMI-1640 medium (Sigma-Aldrich), buffered to $\mathrm{pH}$ 7.0 with MOPS (4-morpholinepropanesulfonic acid, 1.165 M, Sigma-Aldrich), inoculated with $P$. glabrum or A. wentii $\left(0.4 \times 10^{4}\right.$ to $\left.5 \times 10^{4} \mathrm{CFUs} \cdot \mathrm{mL}^{-1}\right)$. The stock solutions of standard compounds were two-fold diluted with RPMI-1640 $(10 \mu \mathrm{L})$, and further 100 -fold diluted with the incolum $(990 \mu \mathrm{L})$ and then placed 
into sterile round-bottomed polystyrene tubes $(12 \times 75 \mathrm{~mm})$ to obtain a set of concentrations from 1,600 to $13.25 \mu \mathrm{g} \cdot \mathrm{mL}^{-1}$ (final volume $=1 \mathrm{~mL}, \mathrm{DMSO} \leq 1 \%$ ). In addition, two types of controls were performed: the growth control (GC, referred as complete inhibition) contained only $1 \%$ the extract solvent ( $10 \mu \mathrm{L}$ of DDW or DMSO); and in the sterile control (SC, referred as no inhibition), uninoculated, plain RPMI-1640 medium was used instead of inoculum. Tubes of extract/compound-inoculum combinations were incubated at $35{ }^{\circ} \mathrm{C}$ in the dark for 48 to 72 hrs until visible growth of fungal colonies appeared on an indicator PDA dish (plated with $10 \mu \mathrm{L}$ of inoculum and incubated with the culture tubes).

After $48 \mathrm{hrs}$ of incubation, $50 \mu \mathrm{L}$ of resazurin sodium solution $\left(100 \mathrm{mg} \cdot \mathrm{L}^{-1}\right.$ with RPMI-1640 medium) was added to each culture tube. Resazurin (7-hydroxy-3Hphenoxazin-3-one-10-oxide sodium salt, Sigma-Aldrich) was used as a reductive dye indicator for cell viability. In the presence of metabolising microorganisms, the blue resazurin (absorbance maximum at $601 \mathrm{~nm}$ ) is reduced to a pink coloured reduced form resorufin (maximum absorbance at $573 \mathrm{~nm}$ ) (Figure 2.6).
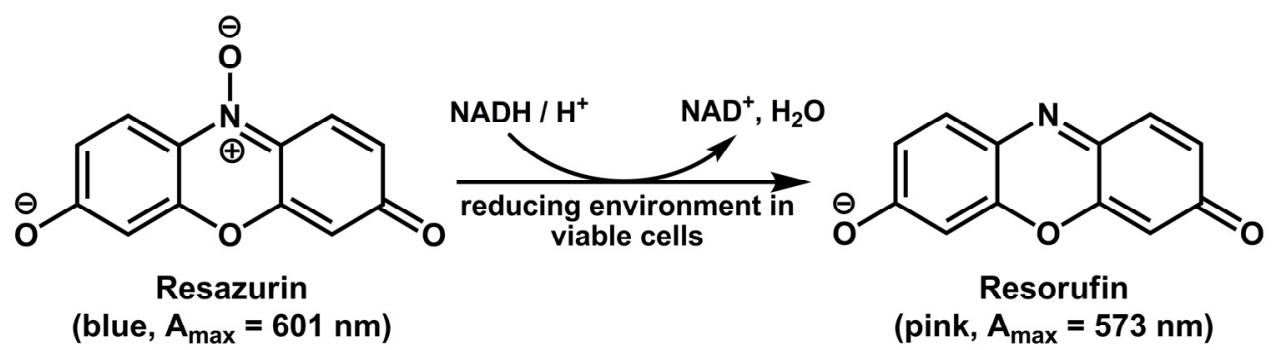

Figure 2.6 Principle of resazurin (sodium salt) in the colorimetric assay.

Fungal growth can therefore be quantified as an absorption spectral colour change with the absorption peak shifting from 601 to $573 \mathrm{~nm}$ as shown in the titration spectra against P. glabrum (Figure 2.7A). To evaluate the extent of antifungal activity, an approximate calibration plot can be constructed showing the absorption maxima ratio $601 \mathrm{~nm}: 573 \mathrm{~nm}$ against the percentage growth inhibition when the GC and SC cultures were mixed in varying amounts (Figure 2.7B). 
Similar results were obtained using $A$. wentii. The data were obtained from only one experiment.
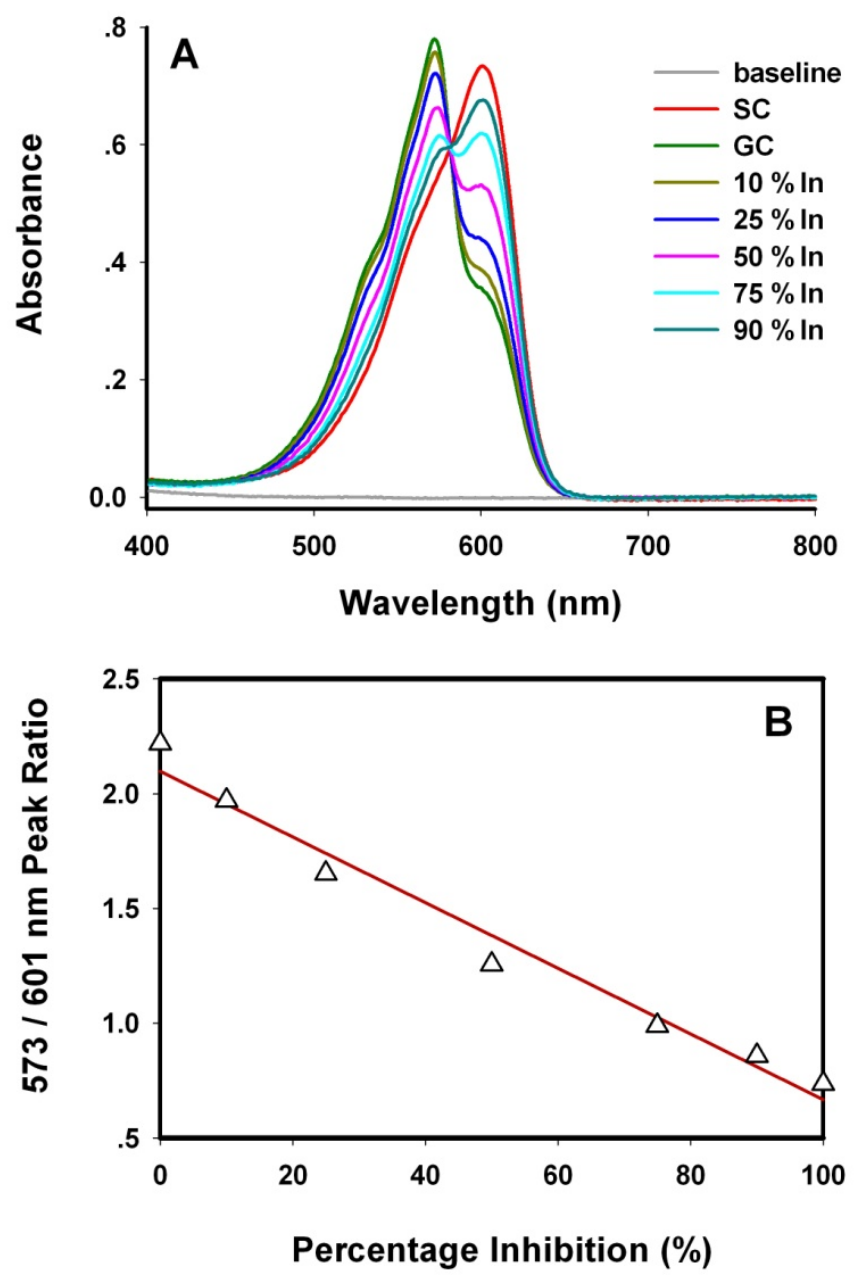

Figure 2.7 An inhibition titration against fungal broth of $P$. glabrum $(10,000$ CFUs' $\mathrm{mL}^{-1}, 48 \mathrm{hrs}$ of incubation) as in absorption spectra (A) and as a calibration curve of peak ratio $601 \mathrm{~nm}: 573 \mathrm{~nm}(\mathrm{~B})$ 


\section{MIC Determination}

Amphotericin B, a widely used antifungal agent with a well-established minimum inhibitory concentration (MIC), was used to validate this resazurin-indicated colorimetric antifungal test. As an important index for fungistatic activity, MIC is defined as the lowest drug concentration that prevents any discernible growth ${ }^{[65]}$.

In the colorimetric test, MIC is referred to the first culture tube showing no perceptible colour change, i.e. remaining blue ${ }^{[65]}$. However, the slight colour change from blue to bluish-purple can sometimes be indiscernible in the conventional visual estimation. As a more objective determination of colour change, the absorption spectra of GC, SC and a set of "potential MIC cultures" (tubes that were visually blue) were measured over the visible wavelength range $(400-800 \mathrm{~nm})$. In the validation with amphotericin B (Figure 2.8), as the drug concentration increase, "MIC cultures" were approaching SC (100\% fungal inhibition). Only the concentration of a complete overlapped spectrum $\left(1 \mu \mathrm{g} \cdot \mathrm{mL}^{-1}\right)$ was regarded as the MIC.

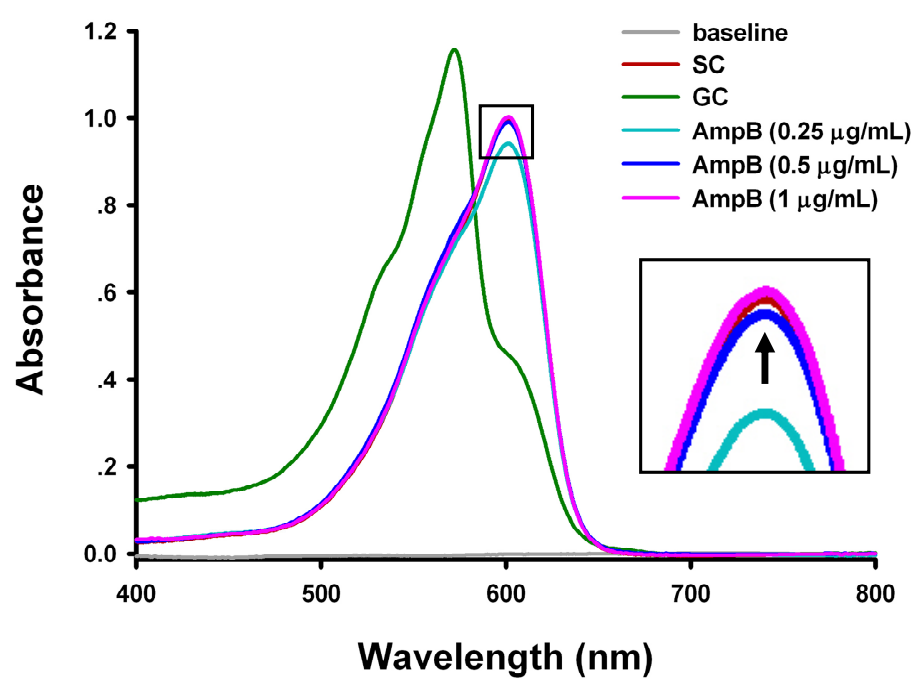

Figure 2.8 Absorption spectra of resazurin-dyed MIC cultures of amphotericin B (AmpB) inoculated with P. glabrum $\left(0.4 \times 10^{4}\right.$ to $\left.5 \times 10^{4} \mathrm{CFUs} \cdot \mathrm{mL}^{-1}\right)$. 


\subsubsection{Results and Discussion}

Table 2.2 shows the in vitro antifungal activities (MIC values in $\mu \mathrm{g} \cdot \mathrm{mL}^{-1}$ ) of amphoterocin B and four commercially-obtained coumarins against two fungal species ( $P$. glabrum and A. wentii). The MICs obtained from the wellcharacterised antifungal agent, amphotericin B, lay in the expected range of previous reports: " 0.5 to $2 \mu \mathrm{g} \cdot \mathrm{mL}^{-1}$ with RPMI-1640 medium for most opportunistic filamentous fungi; and above $2 \mu \mathrm{g} \cdot \mathrm{mL}^{-1}$ for Aspergillus spp." ${ }^{\text {[65, } 68]}$. By contrast, coumarin and 7-hydroxycoumarin (umbelliferone) showed considerable, but weaker, activity (Figure 2.9). However, for the two substituted hydroxycoumarins, 6, 7-dihydroxycoumarin and 7-hydroxy-6-methoxycoumarin, which also caused partial inhibition of fungal growth, complete inhibition was not observed due to their low solubilities in the medium.

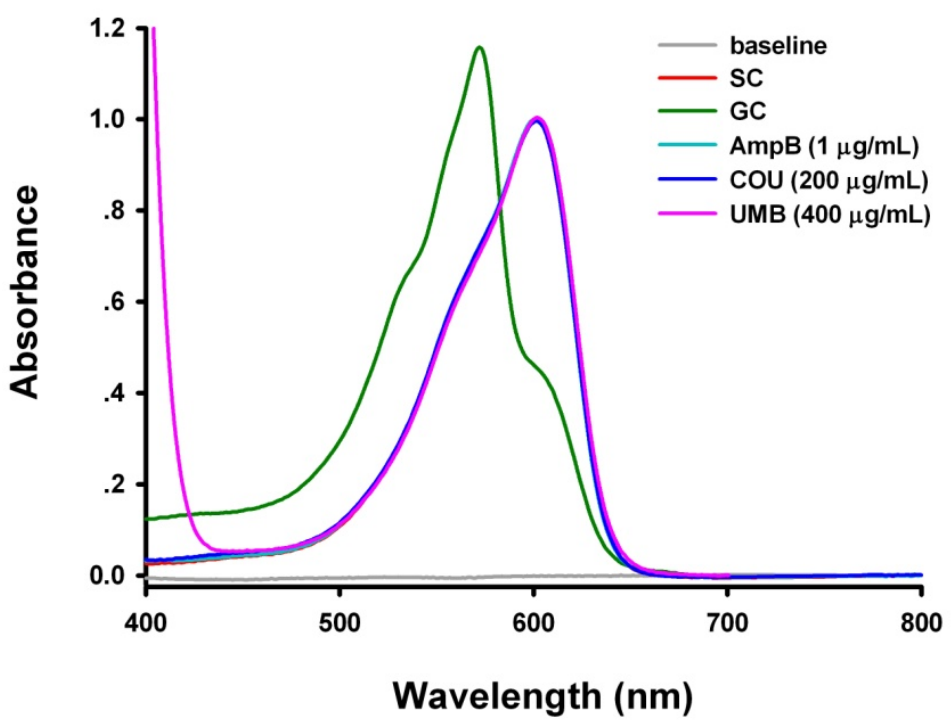

Figure 2.9 Absorption spectra of resazurin-dyed MIC cultures of amphotericin B $(\mathrm{AmpB})$, coumarin (COU) and 7-hydroxycoumarin (UMB) inoculated with $P$. glabrum $\left(0.4 \times 10^{4}\right.$ to $\left.5 \times 10^{4} \mathrm{CFUs} \cdot \mathrm{mL}^{-1}\right)$. 
Table 2.2 In vitro antifungal activity of amphotericin B and four standard coumarins, expressed as MIC $\left(\mu \mathrm{g} \cdot \mathrm{mL}^{-1}\right)$

\begin{tabular}{ccc}
\hline Compound & P. glabrum & A. wentii \\
\hline amphotericin B & 0.5 & 4 \\
coumarin & 200 & 100 \\
7-hydroxycoumarin & 400 & 200 \\
6, 7-dihydroxycoumarin & $>800$ & $>800$ \\
7-hydroxy-6-methoxycoumarin & $>800$ & $>800$ \\
\hline
\end{tabular}

Patterns of substitutions in the phenyl moiety of coumarins were considered to have played a role in the bioactivity. A free 6-hydroxyl group was reported as essential for antifungal activity; and a 7-hydroxylated or alkylated derivatives of coumarin was suggested to have both antifungal and antibacterial properties ${ }^{[69]}$. However, this is not consistent with the results found in this work: hydroxyl substitutions at either position 6 or 7 resulted in weaker rather than enhanced activity, and so did the 6-methoxyl substitution of 7-hydroxycoumarin (scopoletin). The most pronounced effect was demonstrated by the unsubstituted coumarin against $A$. wentii $\left(100 \mu \mathrm{g} \cdot \mathrm{mL}^{-1}\right)$.

Fibres from three cultivars, viz. Arawa, Ngaro and Tapamangu, were extracted in water and the extract partitioned against DCM. The absorption spectra of the aqueous and organic fractions were measured with GC and SC as shown in Figure 2.10. Their inhibition of fungal growth was expressed as the ratio of the absorption spectral maxima, $601 \mathrm{~nm}: 573 \mathrm{~nm}$. Also, the corresponding ratios of the aqueous extracts from the other 11 cultivars of $P$. tenax are shown in Figure 2.11. Their percentage inhibitions were derived from the inhibition titration calibration plot (Figure 2.7). 


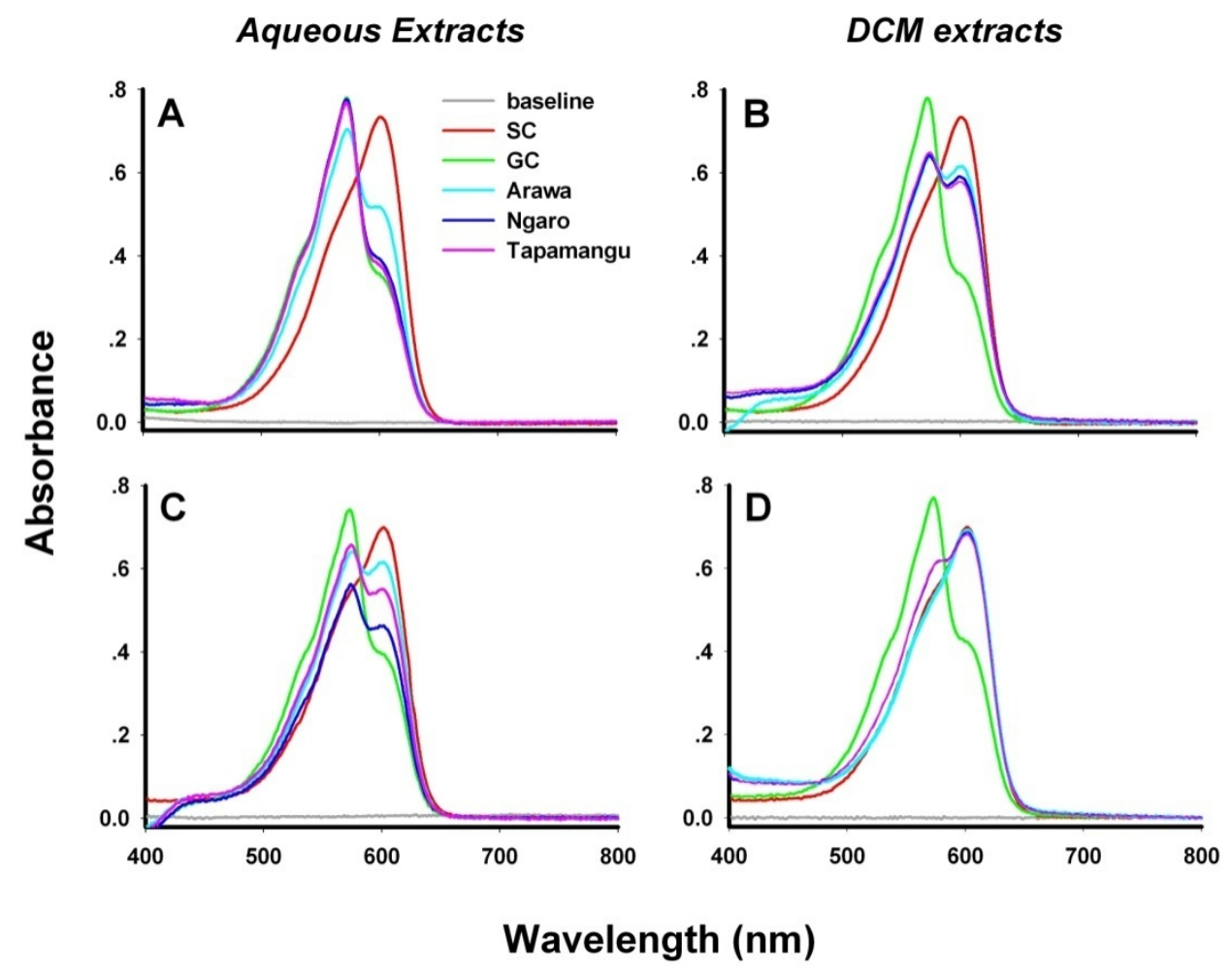

Figure 2.10 Absorption spectra of resazurin-dyed cultures of aqueous (left column) and DCM (right column) extracts from fibres of three cultivars of $P$. tenax against $P$. glabrum ( $\mathrm{A}$ and $\mathrm{B})$ and $A$. wentii $(\mathrm{C}$ and $\mathrm{D})$. Inoculum viability was within the range of $0.4 \times 10^{4}$ to $5 \times 10^{4} \mathrm{CFUs} \cdot \mathrm{mL}^{-1}$. 
The main findings were:

- All aqueous extracts of fibres from the various Phormium cultivars studied in this work showed partial inhibitory effect to fungal growth and were relatively more active against $A$. wentii $(25-75 \%$ inhibition) than $P$. glabrum $(10-50 \%)$.

- There were significant differences in the antifungal activities of aqueous extracts from different Phormium cultivars.

- The antifungal activities of the three DCM extracts were greater than those of their aqueous extracts. This is probably due to the higher concentration of possible antifungal agents in organic solvents. Cultivar Arawa had a MIC $\left(880 \mu \mathrm{g} \cdot \mathrm{mL}^{-1}\right)$ for $A$. wentii, but given that this is the weight of the whole residue, the value cannot be related to a single antifungal agent.

- Among the tested Phormium cultivars, there is no clear correlation between water-extractable antifungal activity (Section 2.3) and the resistance to fungal growth that had shown in the inoculated fibre discs (Section 2.2). For example, of the three cultivars with high resistance in the tests with fibre discs (Section 2.2), Ngaro and Tapamangu, were devoid of antifungal activity in the test of aqueous extracts and only Arawa showed moderate to high activity (Table 2.3). 

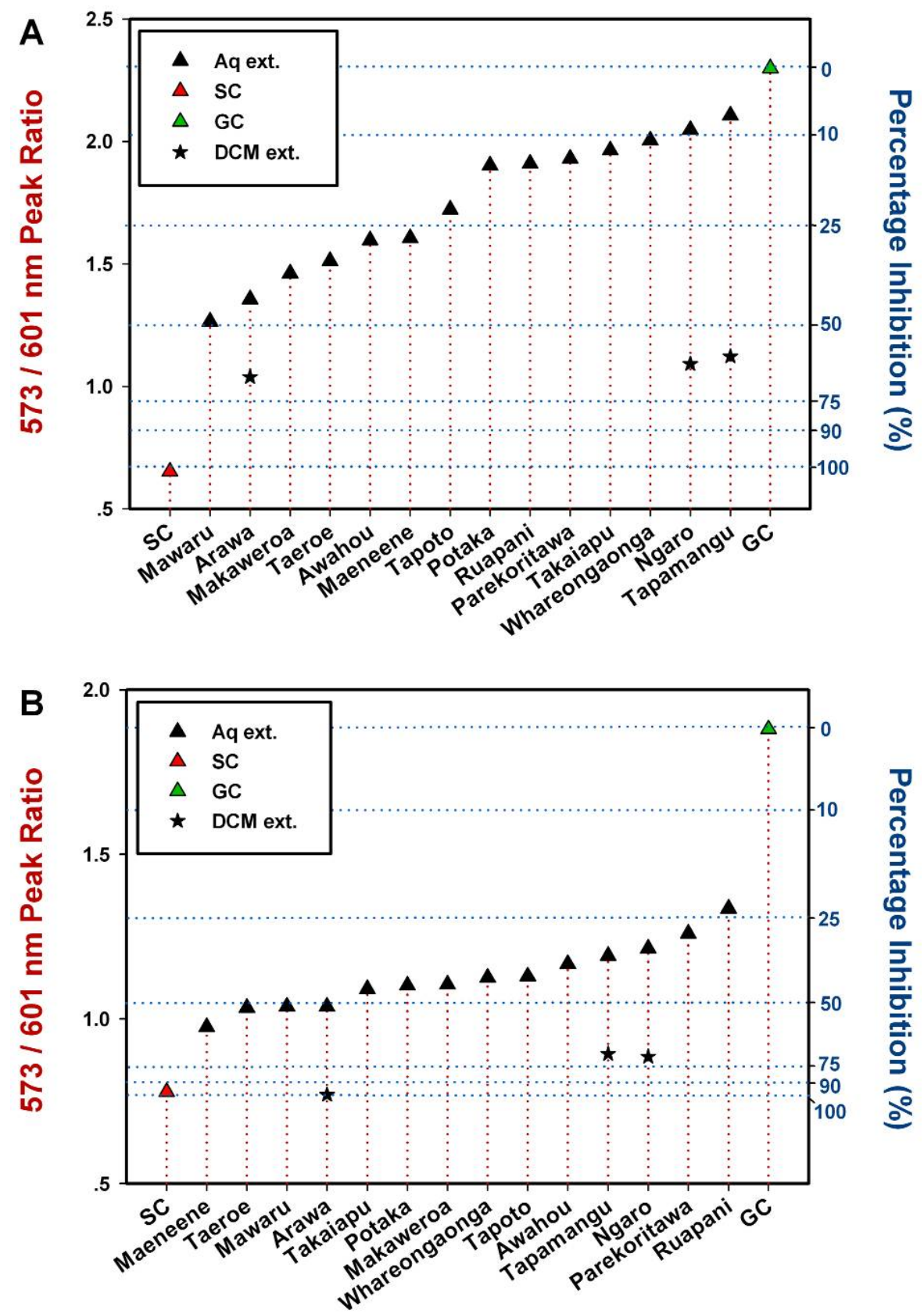

\section{Cultivars of $P$. tenax}

Figure 2.11 Antifungal activities (shown as 573 / $601 \mathrm{~nm}$ peak ratio) of $3 \mathrm{DCM}$ and 14 aqueous extracts of Phormium fibres against $P$. glabrum (A) and $A$. wentii (B). Inoculum viability was within the range of $0.4 \times 10^{4}$ to $5 \times 10^{4} \mathrm{CFUs} \cdot \mathrm{mL}^{-1}$. 
A similar classification of the 14 fibre cultivars studied can be made in which the antifungal activities of the aqueous extracts were divided into three groups: high, moderate and weak (Table 2.3).

Table 2.3 Antifungal activities of aqueous extracts of Phormium fibres from 14 cultivars of $P$. tenax

\begin{tabular}{c|c|c}
\hline \multirow{2}{*}{$\begin{array}{c}\text { *Antifunal } \\
\text { activity }\end{array}$} & \multicolumn{2}{|c}{ Cultivars of $\boldsymbol{P}$. tenax } \\
\cline { 2 - 3 } Low & $\begin{array}{c}\text { Tapoto; Potaka; Ruapani; } \\
\text { Parekoritawa; Whareongaonga; } \\
\text { Takaiapu; Ngaro; Tapamangu }\end{array}$ & A. wentii \\
\hline Moderate & $\begin{array}{c}\text { Mawaru; Arawa; Makaweroa; Taeroe; } \\
\text { Awahou; Maeneene }\end{array}$ & $\begin{array}{c}\text { Takaiapu; Makaweroa; Potaka; } \\
\text { Whareongaonga; Tapoto; } \\
\text { Awahou; Ngaro; Tapmang; } \\
\text { Parekoritawa }\end{array}$ \\
\hline High & - & $\begin{array}{c}\text { Maeneene; Taeroe; Mawaru; } \\
\text { Arawa; }\end{array}$ \\
\hline
\end{tabular}

${ }^{*}$ Low $=\%$ inhibition < 25; Moderate $=\%$ inhibition $25-50$; High = \% inhibition $>50$

In summary, the tested Phormium fibres of 14 cultivars displayed different waterextractable, antifungal activities. The activity can be ascribed to a mixture of antifungal agents that have differing solubilities in water and organic solvents since the inhibitory effect increased significantly for DCM extracted materials that were redisolved in DMSO. The patterns of inhibition against $P$. glabrum and $A$. wentii were slightly different; and extracts were relatively more effective against A. wentii. However, no correlation was found between the antifungal activity of solid fibres and the aqueous extracts, suggesting there are other factors that influence the ability of fibres to resist fungal growth. 


\subsection{Summary of the Chapter}

For the first time, the antifungal properties of Phormium fibres from 14 wellcharacterised cultivars from the National New Zealand Flax collection at Landcare Research were determined. Two fungal species, Penicillium glabrum and Aspergillus wentii, were selected as the test organisms as they are generally considered to be the most important fungal causes of biodeterioration of heritage objects in museums and libraries ${ }^{[54]}$.

In the preliminary susceptibility screening of fungal-infected fibres (see Sec. 2.2), the different cultivars of $P$. tenax tested showed significant differences in the resistance to fungal growth. However, it is not clear from this investigation whether this is due to differences in the amounts or bio-availabilities of antifungal substances or of nutrients present in the fibres. To elucidate this further, the antifungal activities of aqueous extracts of the fibres were evaluated using a standard colorimetric in vitro broth macrodilution method (NCCLS document M38-A $)^{[65]}$. More technical information about these two mycological tests is given in the appendix of this thesis.

Aqueous solutions of four authentic coumarins were also analysed for antifungal activity using the same in vitro methodology. The strongest inhibition of fungal growth was obtained from coumarin itself, while hydroxyl substituents at position 6 or 7 resulted in weaker activity. No correlation was found between the resistance to fungal growth/antifungal activity of solid fibres and the aqueous extracts: cultivars with high resistance in the solid fibre assay exhibited activities ranging from low to high in the in vitro antifungal assay (Table 2.4). This result could be due to the different solubilities of water-extractable, antifungal agents such as coumarins and/or a synergistic action between the agents and other components in the fibre ${ }^{[70]}$. 
Table 2.4 Qualitative evaluation of antifungal activities of Phormium fibres from 14 cultivars of $P$. tenax

\begin{tabular}{c|c|c|c|c}
\hline \multirow{2}{*}{ Phormium Cultivars } & \multicolumn{2}{|c|}{ Solid Fibre* } & \multicolumn{2}{c}{ Aqueous Extract* } \\
\cline { 2 - 5 } & P. glabrum & A. wentii & P. glabrum & A. wentii \\
\hline Parekoritawa & - & & - & + \\
Tapoto & + & + & - & + \\
\hline Arawa & ++ & ++ & + & ++ \\
Makaweroa & + & & - & + \\
Ruapani & + & & - & + \\
\hline Potaka & + & + & - & + \\
\hline Whareongaonga & - & & + & + \\
\hline Tapamangu & ++ & ++ & + & ++ \\
\hline Maeneene & + & + & + & + \\
\hline Mawaru & - & & + & + \\
\hline Awahou & - & - & + & + \\
Takaro & ++ & & + & + \\
\hline Taeore & - & & + & + \\
\hline
\end{tabular}

*Key of antifungal scaling: -, weak/no inhibition; +, moderate inhibition; ++, evident inhibition

To conclude, Phormium fibres from different cultivars differed in their abilities to resist fungal growth, and naturally-occurring coumarins could have played a role in the antifungal properties. However it was not possible to attribute the activity found in the solid fibres/aqueous extracts to a specific compound, or group of compounds. There are other factors likely to contribute to the overall antifungal susceptibility of Phormium fibres and the natural variations, such as nutrient status and/or the presence of other compounds with antifungal activity (e.g. furfural, a degradation product of hemicellulose). Fibres from certain Phormium cultivars (e.g. Arawa and Tapamangu) are intrinsically more resistant to fungal growth than others under humid conditions. This study have provided useful information for fibre selection in creating Phormium heritage objects or textile fibre blends that are protected in situ from fungal attack in a natural, environmentally-friendly way. 


\section{Chapter 3}

\section{Identifying Fluorescent Species in Phormium Fibres}

This chapter covering the identification of fluorescent species in Phormium fibres is divided into two parts. The first part is dedicated to the mass spectroscopic identification of coumarins from the fibres of six Phormium cultivars. In Chapter 2 , five of the six cultivars were shown to possess low to high antifungal activities. As well as antifungal agents, many coumarins also function as fluorescent whitening agents and are suggested to be responsible for the enhanced whiter/brighter appearance and photodegradation of textiles. Therefore, the contribution of naturally-occurring coumarins to the fluorescence of various Phormium fibres is described in the second part of this chapter.

\subsection{Mass Spectrometric Identification}

In this section, identification of coumarins and other compounds (mass range of $50-200 \mathrm{Da})$ important to the antifungal activity of Phormium fibres was performed by electrospray ionisation mass spectrometry (ESI-MS). Compared with the electron impact mass spectrometry (EI-MS), ESI-MS enables rapid and sensitive identification of trace molecules in complex mixtures while avoiding the time-consuming of involved purification procedures and long analysis time. 


\subsubsection{Introduction to ESI-MS Spectrometry}

\section{Electrospray Ionisation (ESI)}

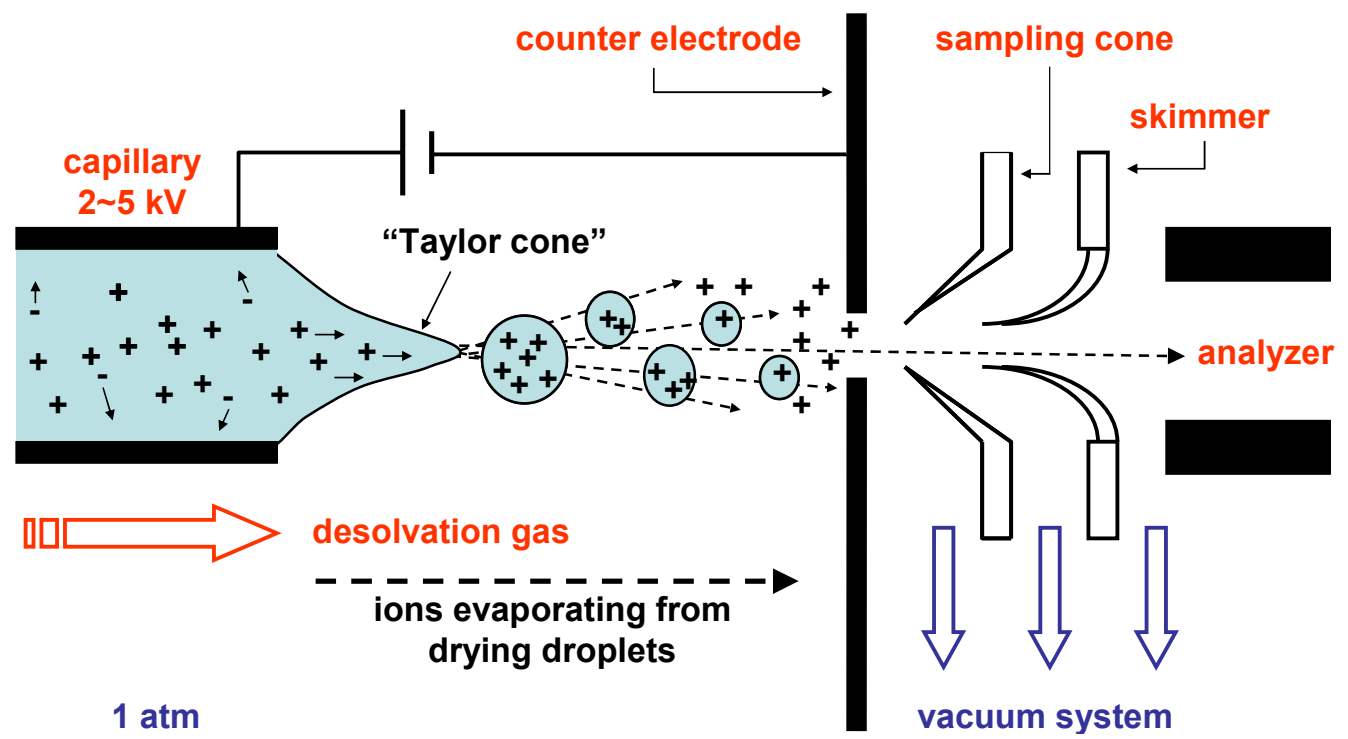

Figure 3.1 Schematic of electrospray ionisation and the desolvation process

In a standard ESI source, the sample to be analysed is introduced in a liquid phase via a capillary as illustrated in Figure 3.1. Under the influence of a strong electric field $(2-5 \mathrm{kV})$ applied between the capillary and a counter-electrode, a continuous flow of analyte (normally $1-10 \mu \mathrm{L} \mathrm{min}{ }^{-1}$ ) elongates into a "Taylor cone", from the end of which, a fine spray of highly charged droplets is developed. The solvent contained in the droplets evaporates in a warm counter-flow of nitrogen gas. The desolvation causes the droplets to shrink and their charge per unit volume (charge density) to increase. When the surface tension is broken, the droplets explode into many daughter droplets ("Coulomb explosion"). This process is repeated many times until bare analyte ions "evaporate" from the surface of the droplets ${ }^{[71]}$. 
ESI is a very "soft" ionisation process. Analytes (M) are introduced in solution and ionised under atmospheric pressure by protonation $(\mathrm{M}+\mathrm{zH})^{\mathrm{z}+}$ in the positive mode, or by deprotonation in a basic solution $(\mathrm{M}-\mathrm{zH})^{\mathrm{z}-}$ in the negative mode. With this ionisation, little fragmentation of the analyte occurs. Small molecules (MW $<1 \mathrm{kDa})$ produce mainly singly charged ions $(\mathrm{z}=1)$, whereas large molecules $(\mathrm{MW}>1 \mathrm{kDa})$ give rise to multiply charged ions $(\mathrm{z}>1)^{[71]}$. The gasphase ions are subsequently drawn into and separated in a mass analyzer through a lensing system. The interface involves a series of skimmer cones between various differentially pumped regions to transport gas and ions from the atmospheric pressure ionisation region to the high vacuum region of MS analyzer ${ }^{[72]}$.

\section{ESI Tandem MS (ESI-MS/MS)}

Tandem mass spectrometry $\left(\mathrm{MS}^{\mathrm{n}}\right)$ involves more than one mass analyzer, often two (MS/MS), separated by a collision cell in which a specific, psuedo-molecular ion is fragmented to provide structural information. The analyzers can be of the same or different types. Increasing in popularity is the instrumentation used in this thesis, i.e. a hybrid quadrupole-time-of-flight (QqTOF) configuration equipped with a nano-ESI source as shown in Figure 3.2.

- The first analyzer (MS-1) is a quadrupole (Q) with two pairs of opposing rods connected to a superimposed radio-frequency potential which produces oscillating electric fields that allow only ions with a selected $\mathrm{m} / \mathrm{z}$ ratio to pass through. Changes to the electric potentials can allow a wide range of $\mathrm{m} / \mathrm{z}$ values to be transmitted.

- The collision cell is an rf-only quadrupole (q), which employs an inert collision gas (e.g. argon) to induce fragmentation, termed collision induced dissociation (CID), of the selected parent ion(s) from MS-1. The directional focusing ability of the rf-only quadrupole maintains fragment ions on a focused axis. 
- The second analyzer (MS-2) is a time-of-flight (TOF) analyzer, which is followed by a multi-channel plate (MCP) detector. In the TOF analyzer, ions separate from each other according to different $\mathrm{m} / \mathrm{z}$ values when travelling along a drift region that is free from electric fields. The MCP detector counts the ions and determines their arrival time to generate a mass spectrum.

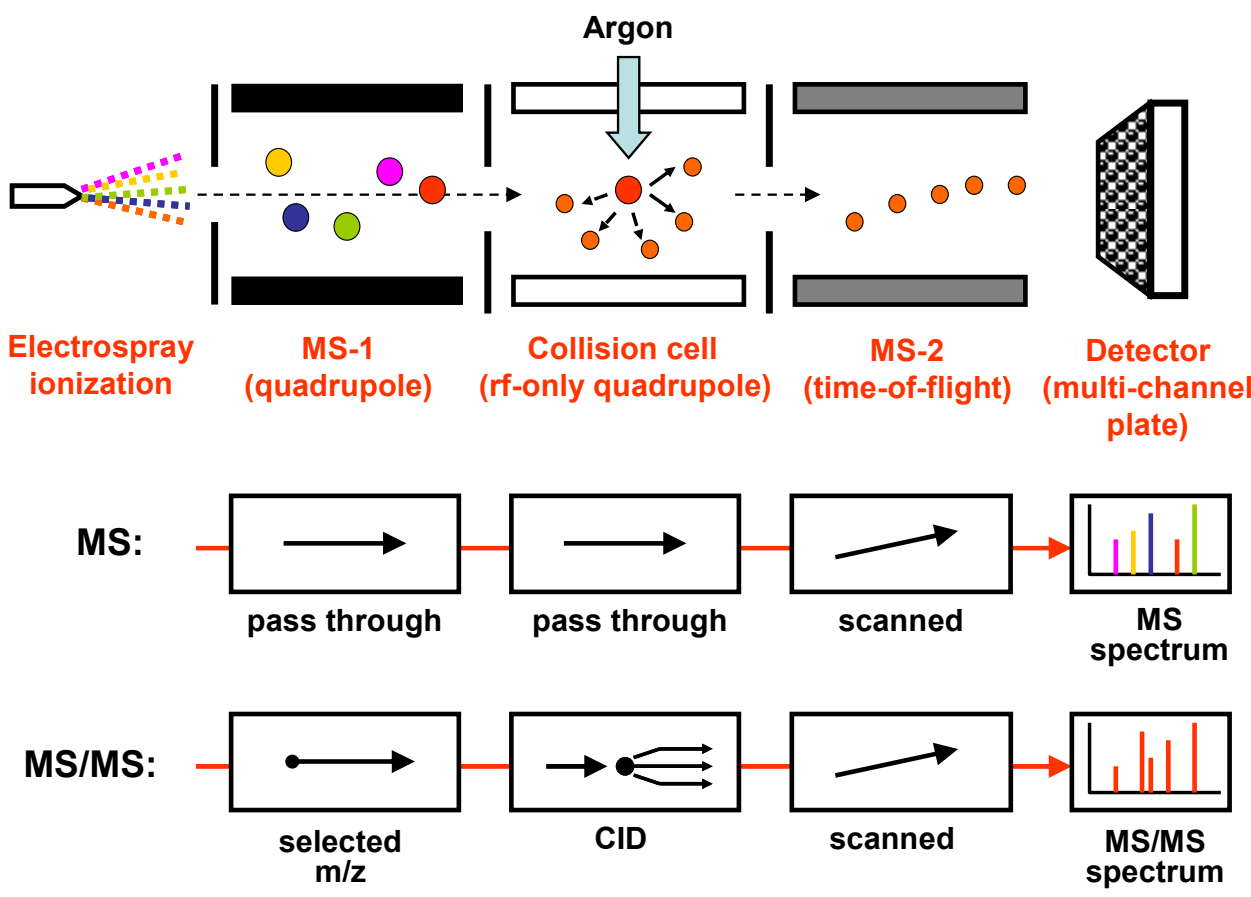

Figure 3.2 Schematic diagram of ESI tandem MS (ESI-MS/MS) and the main processes in the scan modes of MS and MS/MS. CID stands for collision-induced dissociation, as occurs when an inert argon gas is present in the collision cell.

On tandem MS instruments, each stage of MS analysis is carried out sequentially ${ }^{[71]}$. In the MS (or parent ion) scan mode, both MS-1 and the collision cell are set in the rf-only mode, so that all ions, regardless of $\mathrm{m} / \mathrm{z}$ value, are transmitted to the MS-2 for full scanning and a MS spectrum is generated on all intact ions. In the MS/MS (or product ion) mode, the MS-1 acts as mass filter and only a single ion (parent ion) of specific $\mathrm{m} / \mathrm{z}$ value is allowed to enter the collision cell to be fragmented. The resultant fragments are refocused to MS-2 and finally a 
MS/MS spectrum is generated, in which the fragments (daughter ions) observed are derived from the parent ion originally selected by MS-1. The MS/MS spectrum provides valuable structural information of an unknown compound and can also be used as a fingerprint for analytes ${ }^{[73]}$.

\subsubsection{Materials and Methods}

\section{Phormium Fibres and Extraction}

Fibres from the following cultivars of $P$. tenax were investigated: Arawa, Mawaru, Ngaro, Potaka, Tapamangu and Ruawai. Of these, the first five cultivars were shown to have low to high antifungal activities in the mycological screening (see Chapter 2), while the cultivar Ruawai was chosen because it releases a strong and unpleasant odour from its fibres in contrast to the coumarin-like sweet smell of the other cultivars. $0.1 \mathrm{~g}$ of loose fibres from each cultivar were extracted overnight in $0.1 \mathrm{~mL}$ of double distilled water (DDW), assisted by an initial $30 \mathrm{~min}$ of sonication.

\section{Standard Compounds}

Unless otherwise stated, all chemical reagents used in this work were obtained from Sigma-Aldrich. Aqueous solutions of authentic coumarins were prepared in DDW, including coumarin (98\%), 7-hydroxycoumarin (umbelliferone, $99 \%$, Chem Service), 4-methyl-7-hydroxycoumarin (hymecromone, 99 \%), 6,7dihydroxycoumarin (esculetin, $98 \%$ ) and 7-hydroxy-6-methoxycoumarin (scopoletin, 99 \%). Furfural (furan-2-carboxaldehyde) was supplied by Prolabo (Paris, France). All reagents were used without further purification. 


\section{ESI-Mass Spectrometry}

ESI-mass spectrometric analysis was performed using a tandem quadrupole Timeof-Flight (Qq-TOF) mass spectrometer (QSTAR Pulsar-I, Applied Biosystems, Foster City, CA), utilising perfusion nanospray delivery of the aqueous samples. CID utilised argon gas, with collision energies manually adjusted for optimal fragmentation. Positive-ion mode was selected for all analyses.

The aqueous solutions tested, both aqueous extracts and standard compounds, were diluted 1:100 with the eluent $50 \%$ aqueous acetonitrile (Baker analyzed $\AA$ LC/MS Reagent, JT Baker, Phillipsburg, NJ) and $1 \%$ formic acid (Ajax Fine Chemicals, stated purity 99\%) before introduction into the mass spectrometer via nanoflow capillaries (Proxeon Biosystems, Odense, Denmark).

\subsubsection{Results and Discussion}

\section{ESI-MS Analysis}

The ESI-MS was initially set in the MS mode to analyze all singly charged positive ions $\left(\mathrm{MH}^{+}\right)$that were generated from water-soluble extracts. Figure.3.3 shows the MS spectra of the six reference compounds used in the study: furfural, coumarin, 7-hydroxycoumarin (umbelliferone), 4-methyl-7-hydroxycoumarin (hymecromone), 6,7-dihydroxycoumarin (esculetin) and 7-hydroxy-6methoxycoumarin (scopoletin).

Aqueous extracts of Phormium fibres from six cultivars were divided into two groups. Samples of extracts from cultivars Mawaru, Ruawai and Tapmangu were analysed over the range of m/z 90 to 200 and their ESI-MS spectra are shown in Figure 3.4, while the other three cultivars, Arawa, Ngaro and Potaka, shown in Figure 3.5. For all samples, molecular ions with $\mathrm{m} / \mathrm{z}$ corresponding to furfural, coumarin and four 7-hydroxylated derivatives, were marked in the spectra. 


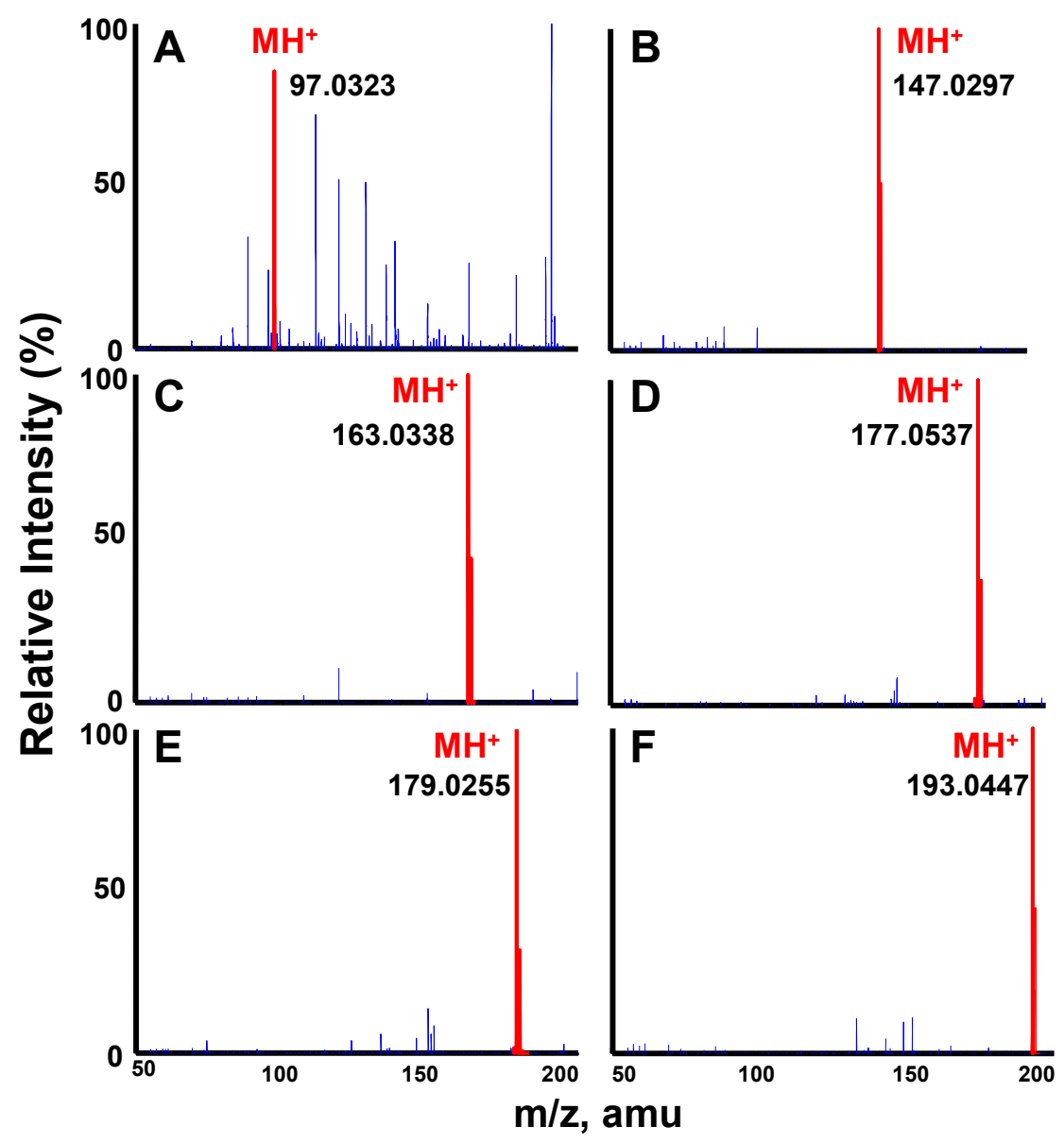

Figure 3.3 ESI-MS spectra of standard furfural (A), coumarin (B), 7hydroxycoumarin $(C)$, 4-methyl-7-hydroxycoumarin $(D), 6,7$-dihydroxycoumarin $(E)$ and 7-hydroxy-6-methoxycoumarin $(F)$, with target molecular ions marked in red. 


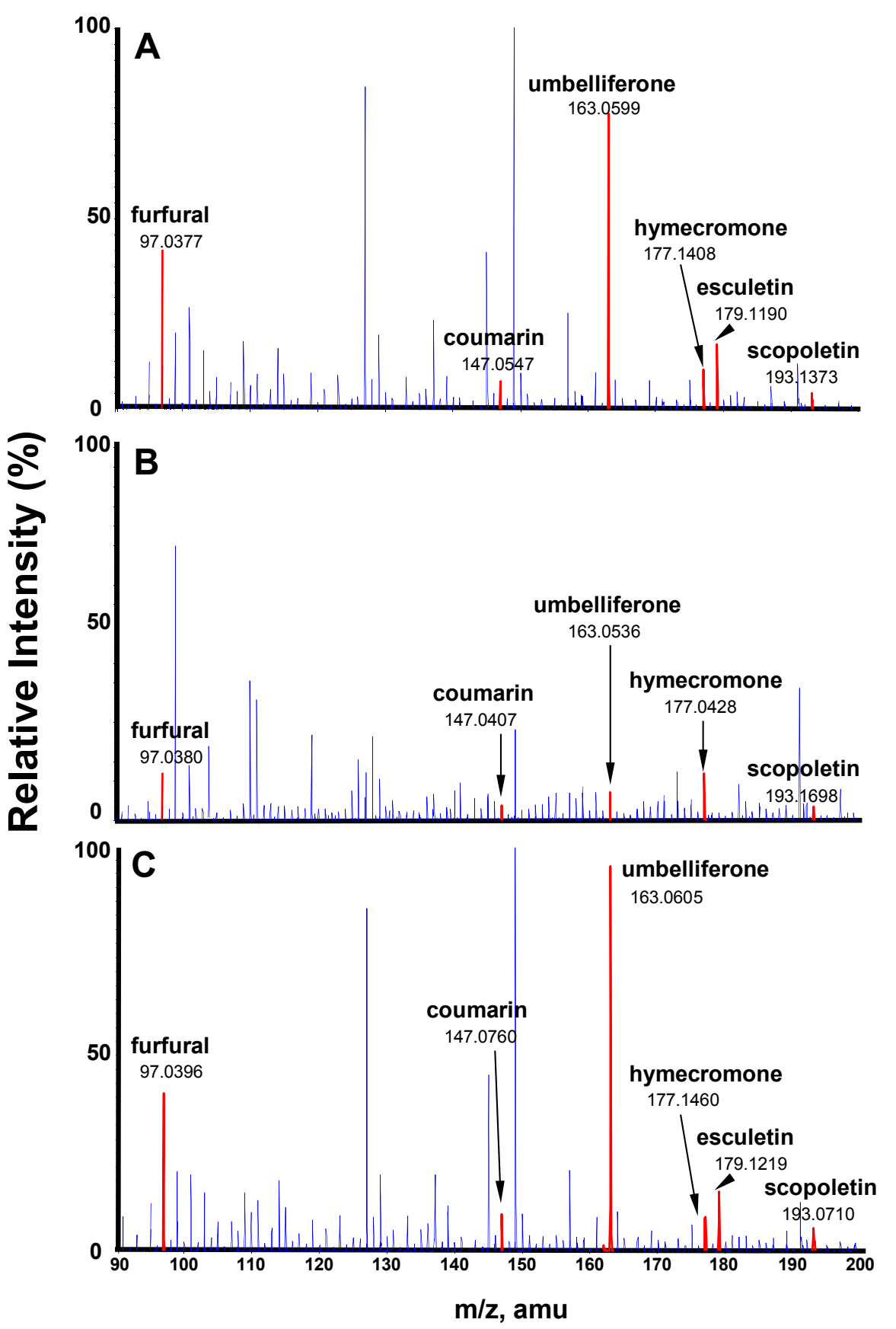

Figure 3.4 ESI-MS spectra of aqueous extracts from Phormium fibres of cultivars Mawaru (A), Ruawai (B) and Tapmangu (C). 


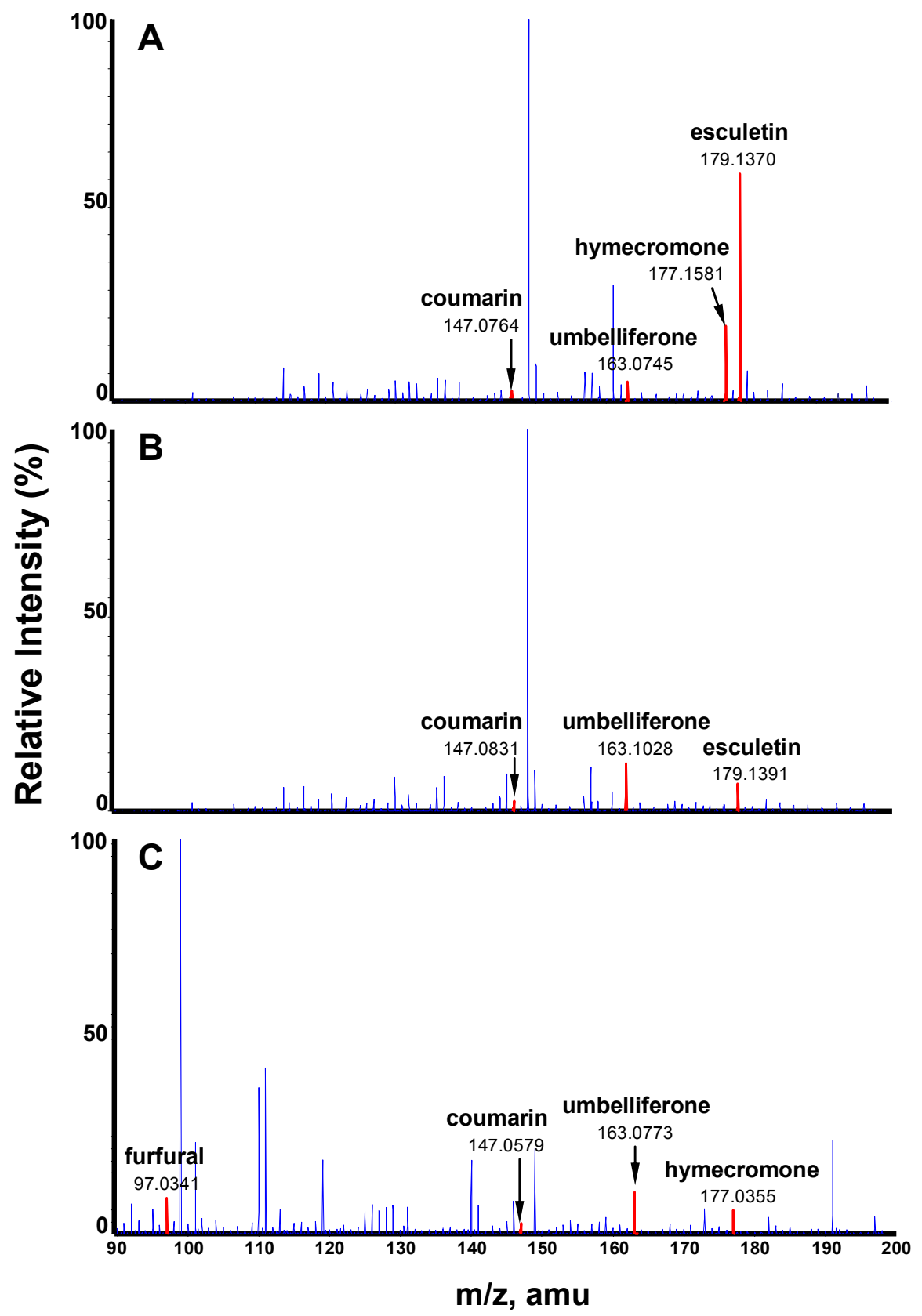

Figure 3.5 ESI-MS spectra of aqueous extracts from Phormium fibres of cultivars Arawa (A), Ngaro (B) and Potaka (C). 
Molecular ions of interest marked in the MS spectra were then selected and fragmented in the MS/MS mode. In a MS/MS spectrum, the elemental compositions of fragment ions and/or neutral loss fragments produced from the parent ion $\mathrm{MH}^{+}$reveals the structural features of the molecule; and the resultant fragmentation pattern may assist with identification of the compound. ESIMS/MS spectra of standard compounds and extractives from Phormium fibres are shown below.

\section{Furfural}

The MS/MS spectrum of standard furfural is shown in Figure 3.6 (top), with that from an aqueous extract of the cultivar Mawaru shown in the bottom. For the remainder of this section, only the ESI-MS/MS spectrum of aqueous extract of Mawaru will be discussed together with that of a reference compound. For furfural, the three characteristic fragment ions may have derived from the loss of the small neutral molecules $\mathrm{H}_{2} \mathrm{O}, \mathrm{CO}$ and $\mathrm{C}_{2} \mathrm{H}_{2} \mathrm{O}$, at $\mathrm{m} / \mathrm{z} 79,69,55$, respectively; and since they were observed in both the sample and the reference spectrum, it is very probable that furfural is a component of the extract of this fibre cultivar.

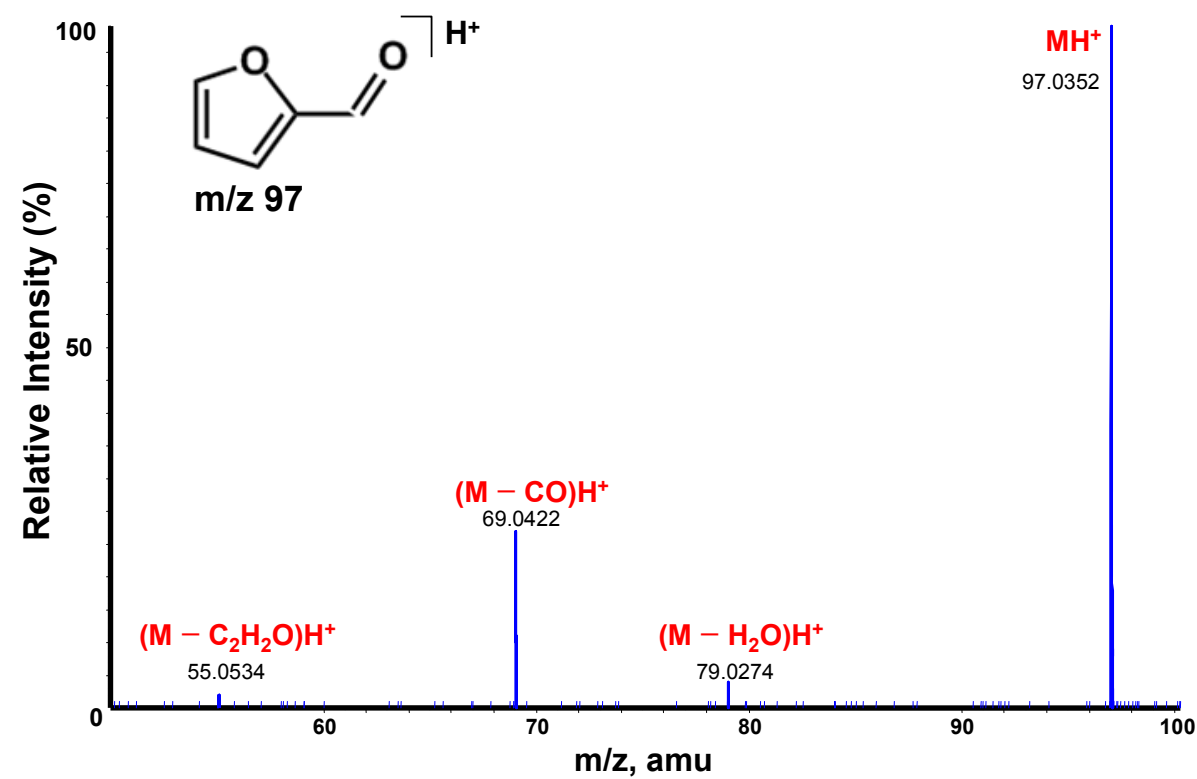




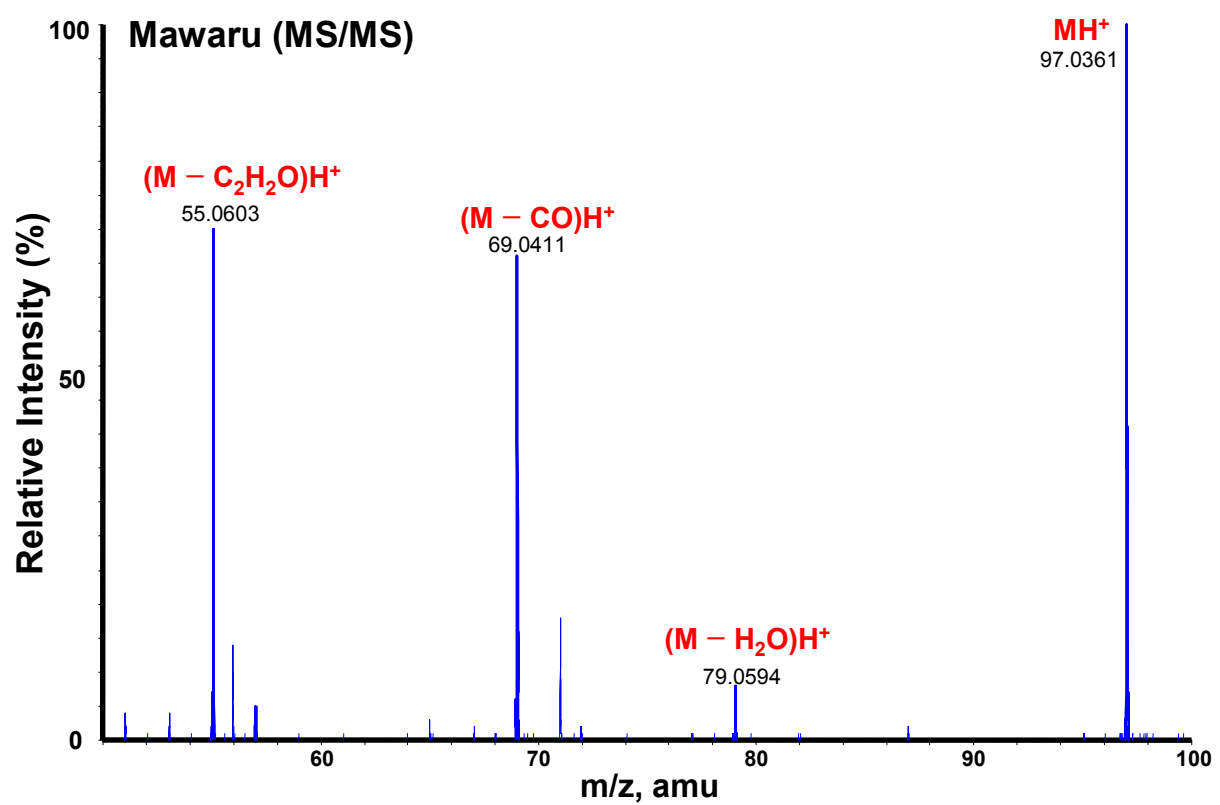

Figure 3.6 ESI-MS/MS spectra of standard furfural (top) and the aqueous extract of Mawaru (bottom), with characteristic fragments labelled.

\section{Coumarin}

The MS/MS spectrum of coumarin is shown in Figure 3.7 (top). There were two daughter ions at m/z 103 and 91, corresponding to the loss of $\mathrm{CO}_{2}$ and $\mathrm{C}_{2} \mathrm{O}_{4}$ $\left(2 \mathrm{CO}_{2}\right)$, respectively. In the MS/MS spectrum of the Mawaru extract, another daughter ion at m/z 119 was present which corresponds to the loss of $\mathrm{CO}$, and were reported in the EI-MS spectrum of coumarin ${ }^{[74]}$. Smaller fragments at $\mathrm{m} / \mathrm{z} 84$ and 69 were also observed, but their exact structure is unknown. The fragmentation pattern observed in the MS/MS of Mawaru extract suggests that coumarin is a component of the extract, while the differences between that and the $\mathrm{MS} / \mathrm{MS}$ of the reference coumarin could be due to cations from other components of the aqueous extract of natural fibre. 

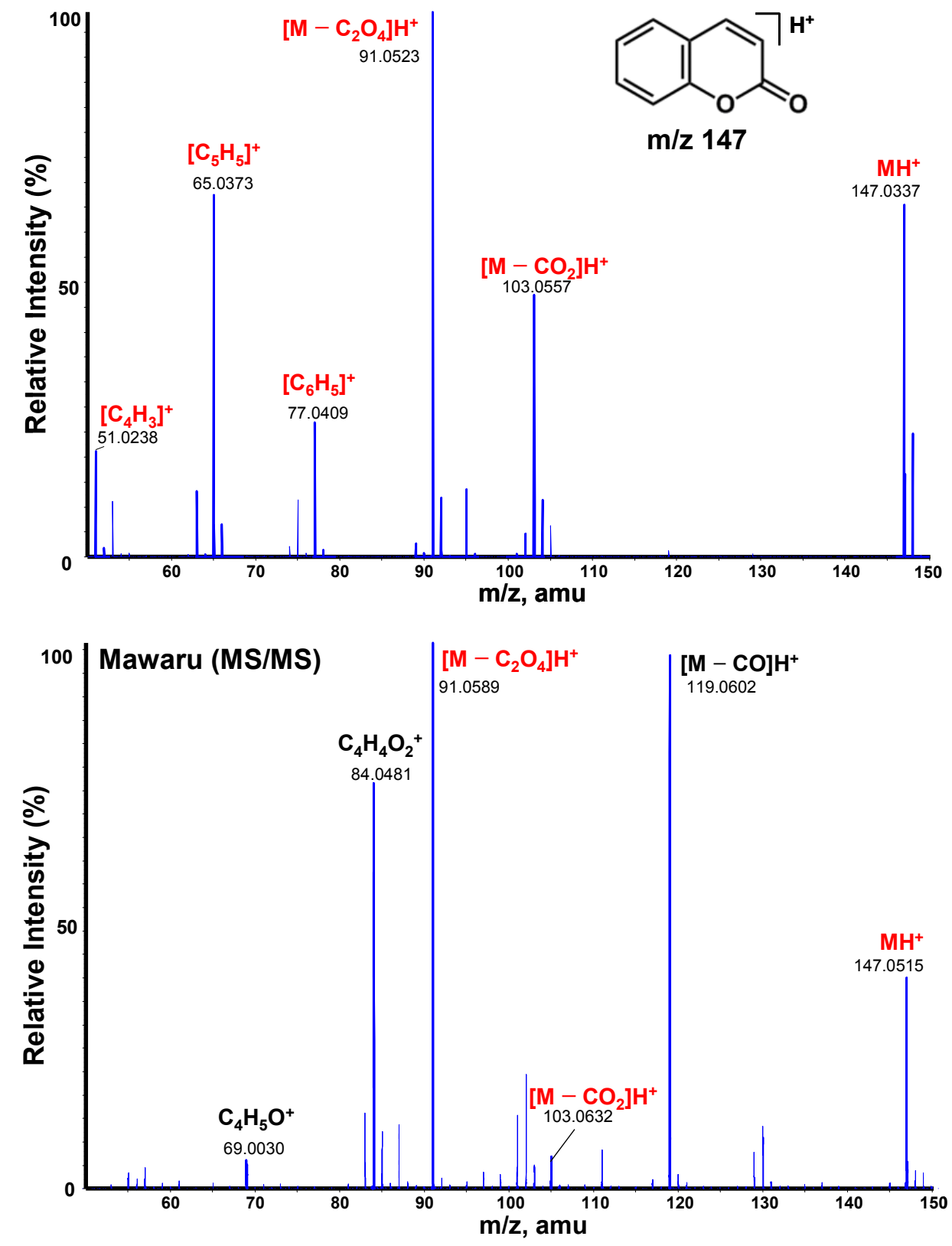

Figure 3.7 ESI-MS/MS spectra of standard coumarin (top) and the aqueous extract of Mawaru (bottom), with characteristic fragments labelled

\section{7-Hydroxycoumarin (Umbelliferone)}

The MS/MS of this compound is shown in Figure 3.8. In addition to the fragments characteristic of coumarin, daughter ions at m/z 135 and 107 from the loss of CO 
and $\mathrm{C}_{2} \mathrm{O}_{2}(2 \mathrm{CO})$ were also present. In the MS/MS spectrum of Mawaru extract, there are additional ions at $\mathrm{m} / \mathrm{z} 145,117$ and 89 from the loss of $\mathrm{H}_{2} \mathrm{O}, \mathrm{CH}_{2} \mathrm{O}_{2}(\mathrm{CO}$ $\left.+\mathrm{H}_{2} \mathrm{O}\right)$ and $\mathrm{C}_{2} \mathrm{H}_{2} \mathrm{O}_{3}\left(\mathrm{C}_{2} \mathrm{O}_{2}+\mathrm{H}_{2} \mathrm{O}\right)$ which were not seen in the reference.
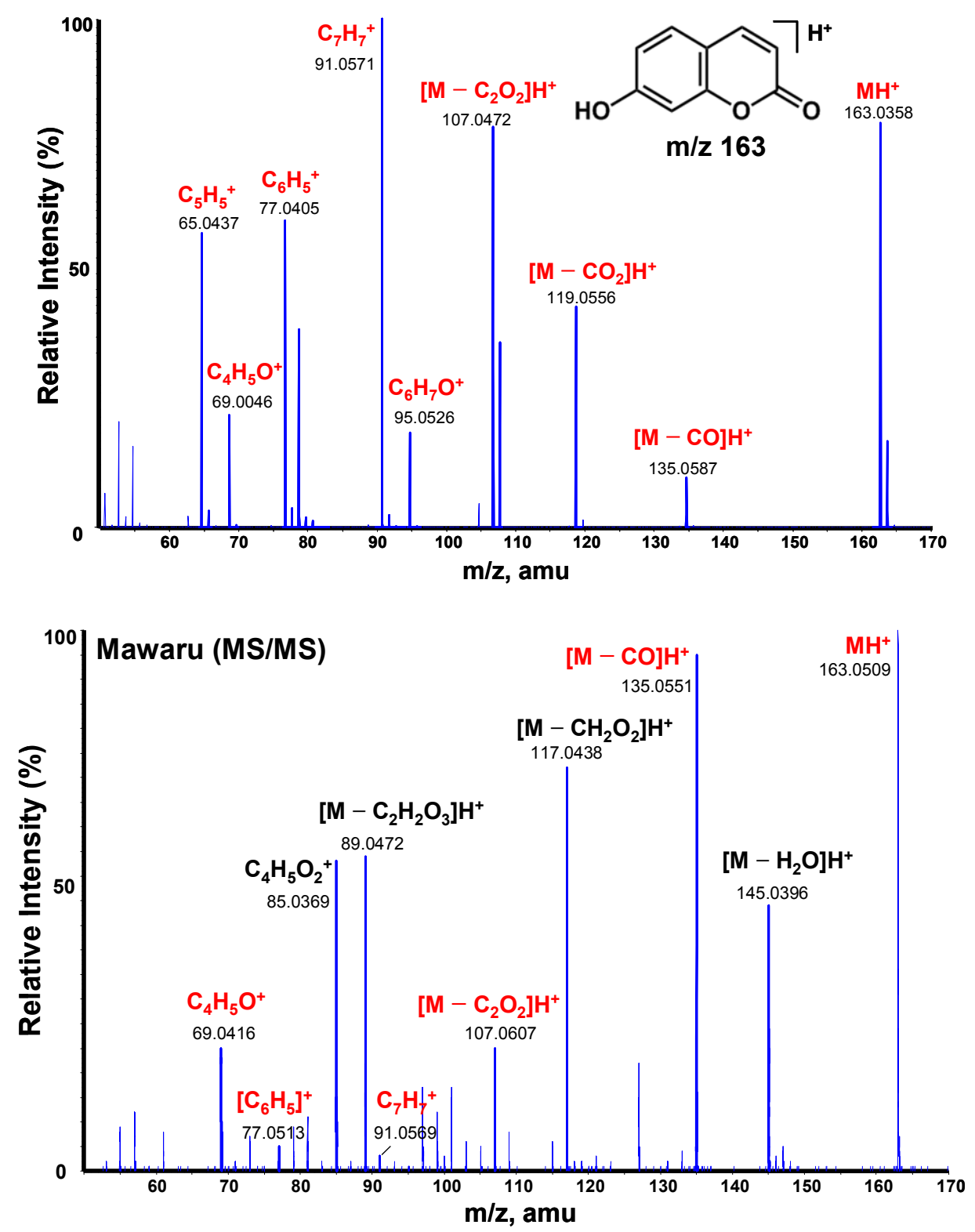

Figure 3.8 ESI-MS/MS spectra of 7-hydroxycoumarin (top) and the aqueous extract of Mawaru (bottom), with characteristic fragments labelled 


\section{7-Hydroxy-4-Methylcoumarin (Hymecromone)}

In the MS/MS spectrum of the Mawaru extract shown in Figure 3.9 (bottom), the additional methyl group of this compound produced a daughter ion at m/z 134 resulting from the loss of $\mathrm{C}_{2} \mathrm{H}_{3} \mathrm{O}\left(\mathrm{CH}_{3}+\mathrm{CO}\right)$ in contrast with 7-hydroxycoumarin (Figure 3.8).
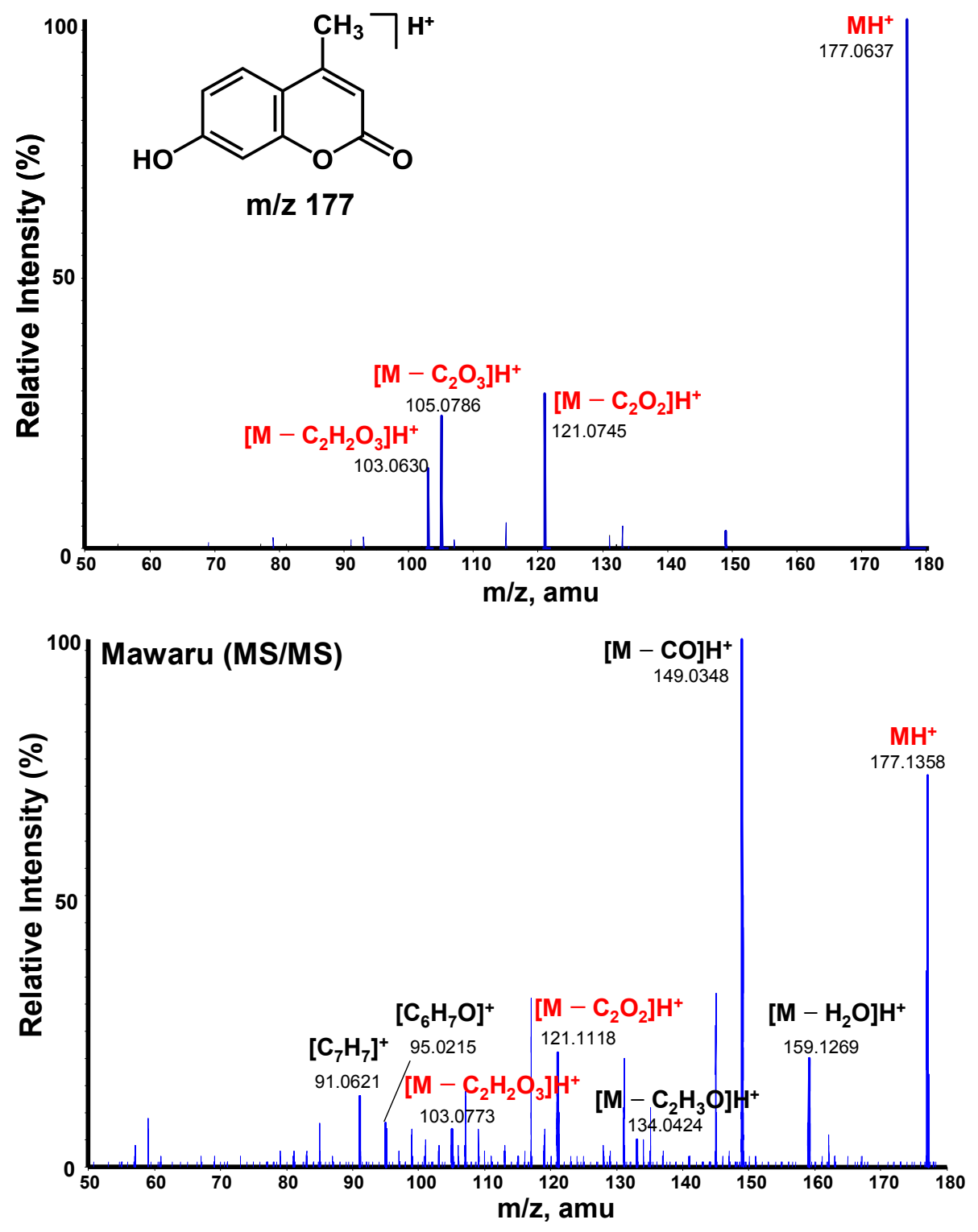

Figure 3.9 ESI-MS/MS spectra of 7-hydroxy-4-methylcoumarin (top) and the aqueous extract of Mawaru (bottom), with characteristic fragments labelled. 


\section{6,7-Dihydroxycoumarin (Esculetin)}

As shown in Figure 3.10, the fragmentation of this dihydroxycoumarin (top) was essentially the same as that of 7-hydroxycoumarin (Figure 3.8), but with an additional oxygen in each fragment. These ions were also seen in the Mawaru extract in the bottom of the figure, thus confirming the presence of this compound.
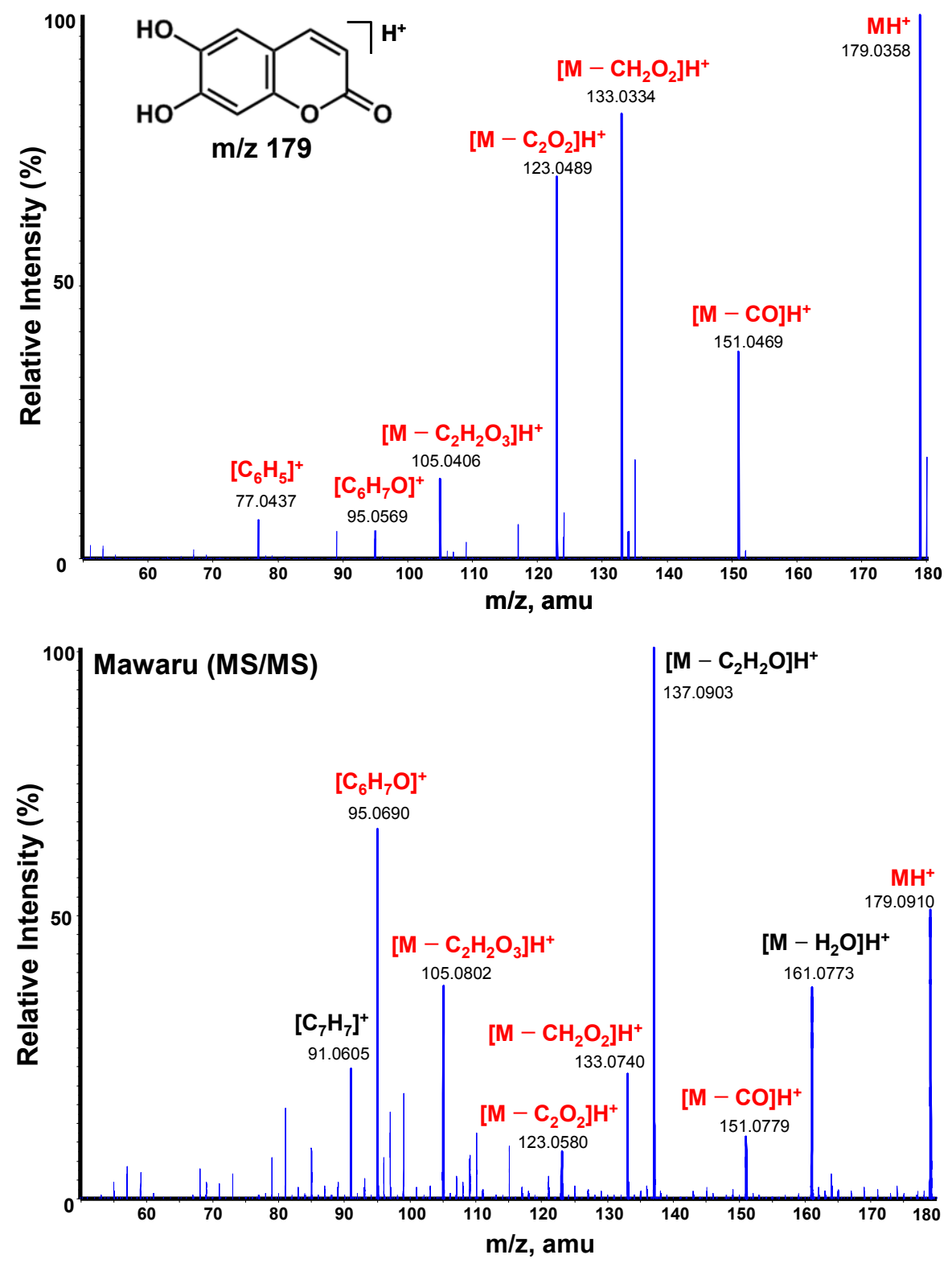

Figure 3.10 ESI-MS/MS spectra of 6,7-dihydroxycoumarin (top) and the aqueous extract of Mawaru (bottom), with characteristic fragments labelled. 


\section{7-Hydroxy-6-Methoxycoumarin (Scopoletin)}

In the MS/MS of 7-hydroxy-6-methoxycoumarin, two characteristic ions were seen at m/z 133 and 105 which could arise from the loss of $\mathrm{C}_{2} \mathrm{H}_{4} \mathrm{O}_{2}$ (acetic acid $\left.\mathrm{CH}_{3} \mathrm{COOH}\right)$ and $\mathrm{C}_{3} \mathrm{H}_{4} \mathrm{O}_{3}\left(\mathrm{CH}_{3} \mathrm{COOH}+\mathrm{CO}\right)$. These ions were present in the MS/MS spectra of both Mawaru extract and reference (Figure 3.11).
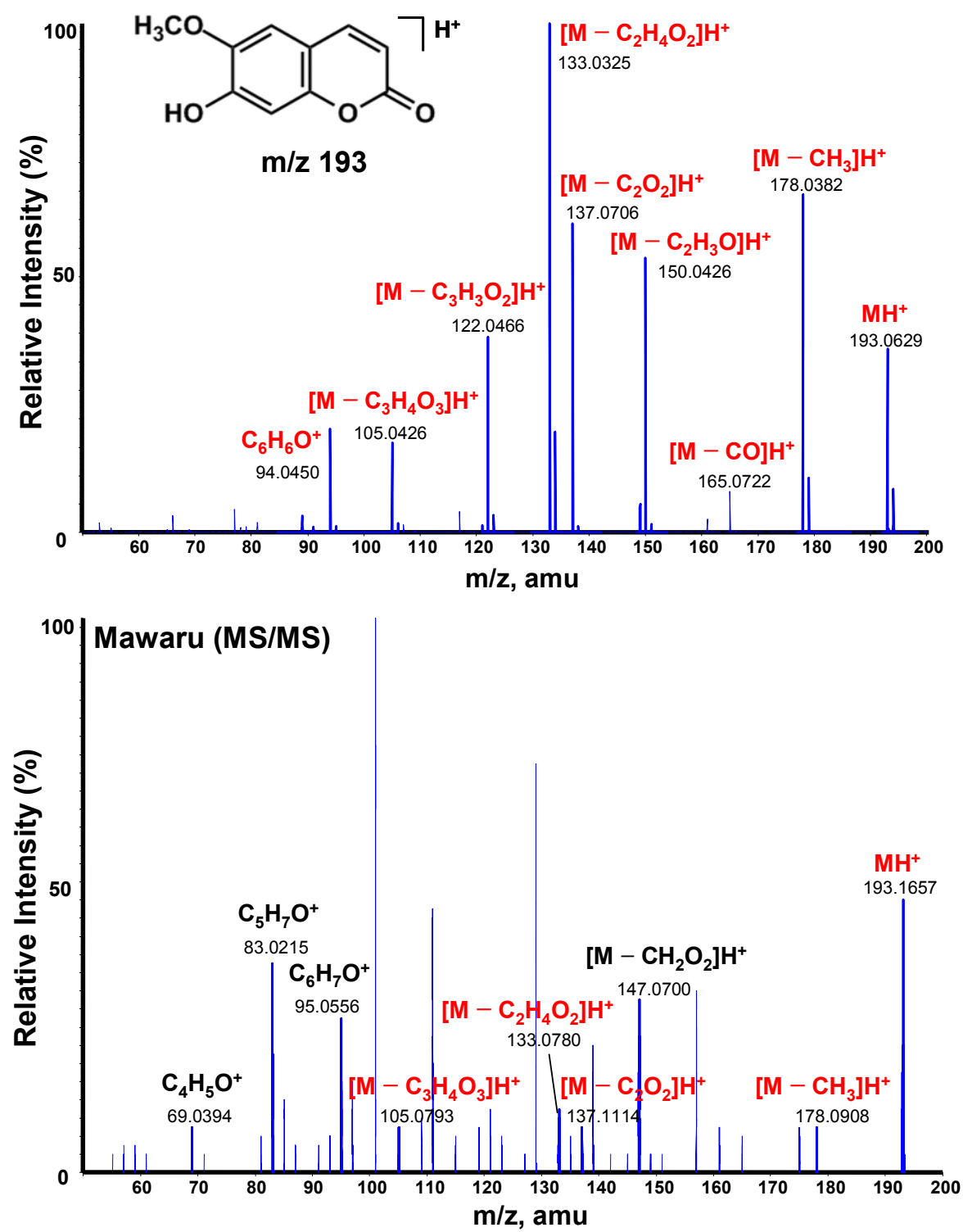

Figure 3.11 ESI-MS/MS spectrum of 7-hydroxy-6-methoxycoumarin and aqueous extract of Mawaru (bottom), with characteristic fragments labelled. 
Table 3.1 Diagnostic daughter ions in ESI-MS/MS characterisation of aqueous extracts of Phormium fibres by reference to authentic compounds. Ions are those resulting from the loss of neutral molecules from $\mathrm{MH}^{+}$and/or are characteristic fragments of the structure.

\begin{tabular}{|c|c|c|c|c|c|c|c|c|c|c|c|c|c|c|}
\hline \multirow[t]{2}{*}{ Compound } & \multirow{2}{*}{$\begin{array}{l}\mathrm{MH}^{+} \\
\mathrm{m} / \mathbf{z} \\
\end{array}$} & \multicolumn{8}{|c|}{ Neutral Loss Units } & \multicolumn{5}{|c|}{ Fragment Ions } \\
\hline & & $\mathrm{CH}_{3 \sim 4}$ & $\mathrm{H}_{2} \mathrm{O}$ & CO & $\mathrm{CO}_{2}$ & $\mathrm{CH}_{2} \mathrm{O}_{2}$ & $\mathrm{C}_{2} \mathrm{H}_{2} \mathrm{O}$ & $\mathrm{C}_{2} \mathrm{H}_{3} \mathrm{O}$ & $\mathrm{C}_{2} \mathrm{O}_{2}$ & $\mathrm{C}_{2} \mathrm{H}_{4} \mathrm{O}_{2}$ & $\mathrm{C}_{7} \mathrm{H}_{5} \mathrm{O}^{+}$ & $\mathrm{C}_{6} \mathrm{H}_{7} \mathrm{O}^{+}$ & $\mathrm{C}_{7} \mathrm{H}_{7}^{+}$ & $\mathrm{C}_{4} \mathrm{H}_{5} \mathrm{O}^{+}$ \\
\hline Furfural & 97 & & 79 & 69 & & & 55 & & & & & & & 69 \\
\hline Coumarin & 147 & & & 119 & 103 & & & & & & & & 91 & 69 \\
\hline 7-hydroxycoumarin & 163 & & 145 & 135 & & 117 & & & 107 & & & & 91 & 69 \\
\hline 7-hydroxy-4-methylcoumarin & 177 & & 159 & 149 & & & & 134 & 121 & & 105 & 95 & 91 & \\
\hline 7-hydroxy-6-methoxycoumarin & 193 & 178 & & & & & & & 137 & 133 & 105 & 95 & & 69 \\
\hline
\end{tabular}

Note: the ions of $\mathrm{m} / \mathrm{z}$ values marked in red are observed in the MS/MS spectra of both aqueous extracts of Phormium fibres and the standard compound. 
The diagnostic fragment ions used for structural characterisation in this study are summarised in Table 3.1. The obtained fragmentation patterns are in good agreement with literature ${ }^{[74,75]}$. This work provides strong evidence for the occurrence of the natural products listed in Table 3.2 in aqueous extracts of Phormium fibres of the six studied cultivars.

Table 3.2 ESI-MS/MS identification of selected products in aqueous extracts from fibres from six cultivars of $P$. tenax

\begin{tabular}{cccccccc}
\hline Compound $^{*}$ & \multicolumn{7}{c}{ Cultivars of $\boldsymbol{P}$. tenax } \\
\cline { 2 - 6 } & Mawaru & Ruawai & Tapamangu & Arawa & Ngaro & Potaka \\
\hline furfural & + & + & + & - & - & + \\
coumarin & + & + & + & + & + & + \\
7-hydroxycoumarin & + & + & + & + & + & + \\
7-hydroxy-4-methylcoumarin & + & + & + & + & - & + \\
6,7-dihydroxycoumarin & + & - & + & + & + & - \\
7-hydroxy-6-methoxycoumarin & + & + & + & - & - & - \\
\hline
\end{tabular}

*Presence of compound is indicated by + ; its absence is indicated by -

It is of interest to note that Mawaru, the most resistant cultivar of $P$. tenax in the aqueous antifungal assay (see Chapter 2, Section 2.3) also possessed the greatest number of possible antifungal agents as determined in this work. These products could possibly exert a synergistic antifungal effect as previously reported on coumarins and other compounds that occur in higher plants ${ }^{[59,70]}$. Also notable is the volatility of the detected compounds: furfural is volatile; coumarin and hydroxycoumarins are semi-volatile with a sweet smell of new-mown hay. All of these products may arise by enzymatic reactions, on precursors in the Phormium fibres that are initiated by microbial attack. Interestingly in history, the fungus Botrytis cinerea, also known as "noble rot", was utilised to induce the release of volatile aroma compounds in grapes to produce the unique flavour of a French wine, Sauternes ${ }^{[76]}$. 


\subsection{Fluorescence of Phormium Fibres}

\subsubsection{Electronic Excited States and Fluorescence}

The different energetic levels (states) and transitions in a photo-excited molecule can be illustrated conveniently in a Jablonski diagram as shown in Figure 3.12. The molecular electronic states (thick horizontal lines) are arranged vertically in increasing levels of energy and grouped horizontally by different electron spin multiplicity: singlet states where the highest energy pair of electron spins are antiparallel and are denoted $S_{0}$ (ground state), $S_{1}$ (first excited state) and the first triplet state $T_{1}$ where the highest energy electron pair spins are parallel. Vibrational levels (thin horizontal lines) are substates associated with each electronic state.

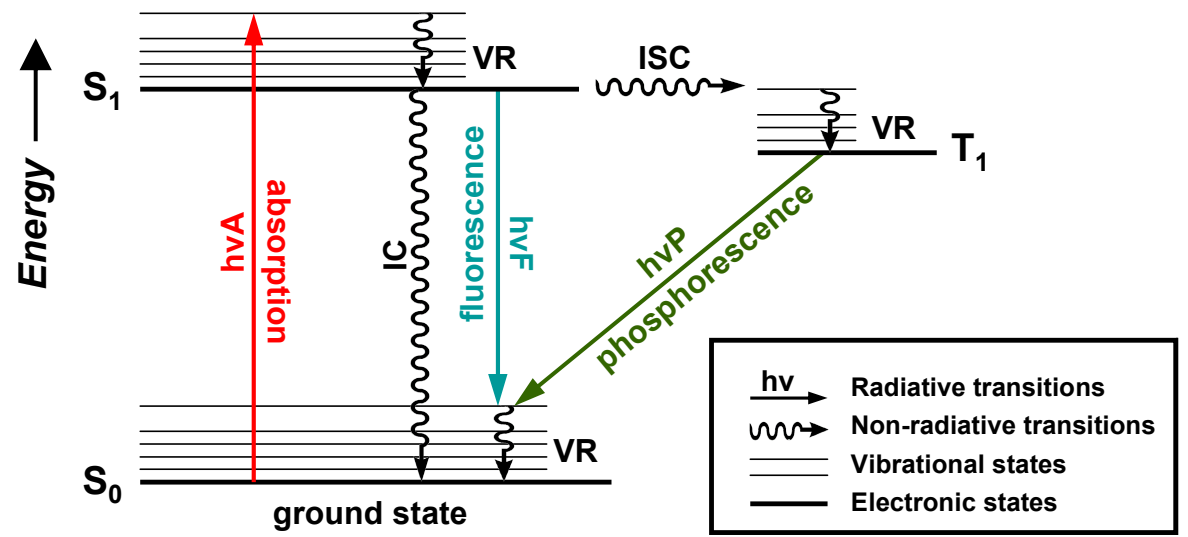

Figure 3.12 A Jablonski diagram in which the molecular energetic levels are depicted by horizontal lines, non-radiative transitions are indicated by wave arrows and radiative transitions by straight arrows. 


\section{Absorption of UV-Visible Light}

The Stark-Einstein principle states that a molecule can only absorb one quantum of radiation viz. a photon (hv) at a time ${ }^{[77]}$ :

$$
\mathrm{M}+\mathrm{h} v \rightarrow \mathrm{M}^{*}
$$

where the $\mathrm{h}$ is Planck's constant; and since $v=\mathrm{c} / \lambda$ (frequency $=$ speed of light $/$ wavelength), each such optical transition occurs with a specific energy and thus at a specific wavelength (energy). Therefore, only when absorbing a photon of appropriate wavelength, will a molecule in the ground state $(\mathrm{M})$ be excited $\left(10^{15} \cdot \mathrm{s}^{-}\right.$ $\left.{ }^{1}\right)$ to an excited state of higher energy $\left(\mathrm{M}^{*}\right)$, wherein usually an electron from a bonding $(\sigma, \pi)$ or nonbonding (n) orbital is promoted to a unoccupied antibonding orbital $\left(\pi^{*}\right.$ or $\left.\sigma^{*}\right)$. In the case of most organic molecules, the energy difference between the ground and excited states is such that the wavelength of the absorbed photon falls in the ultraviolet-visible region, and only transitions of the type $\pi \rightarrow$ $\pi^{*}$ and $\mathrm{n} \rightarrow \pi^{*}$ are involved.

As shown in Figure 3.12, the vertical arrow of absorption occurs from the ground state of $\mathrm{S}_{0}$ to one of the vibrational levels of $\mathrm{S}_{1}$. Since at most temperatures, the numbers of vibrational states are large, absorption spectra are generally broadened as a band extending over a range of wavelengths/energies rather than a single line. The $\mathrm{S}_{0} \rightarrow \mathrm{S}_{1}$ transition is the only one that occurs because it is spin allowed and most organic molecules exist as ground-state singlets (molecular oxygen is a rare example of a triplet ground electronic state).

\section{Excited State Transitions and Relaxation}

A molecule in the excited state is unstable and can return rapidly to the ground state via three competing de-excitation pathways ${ }^{[78]}$ :

- Non-radiative transitions (wave arrows) - the absorbed energy is rapidly dissipated into heat by collisions with surrounding solvent molecules. 


$$
\mathrm{M}^{*} \rightarrow \mathrm{M}+\text { heat }
$$

In fact, there are three steps involved in this radiationless decay. First, vibrational relaxations (VR) dissipate the excess vibrational energy, and the excited state of molecule returns to its lowest vibrational energy level. This is the first step for all de-excitation process. Then, a singlet excited molecule may undergo internal conversion (IC) to the ground state of same spin multiplicity $\left(\mathrm{S}_{1} \rightarrow \mathrm{S}_{0}\right.$ ), or by intersystem crossing (ISC) into a metastable state of different multiplicity e.g. a triplet state $\left(S_{1} \rightarrow T_{1}\right)$, but the latter is a spin-forbidden conversion and therefore is much slower. Again, VR occurs leading to the lowest vibrational level of the triplet state.

- Radiative transitions (straight arrows) - the energy, from the lowest vibrational level of an excited state, may be lost by emission of a photon:

$$
\mathrm{M}^{*} \rightarrow \mathrm{M}+\mathrm{h} v^{\prime}
$$

This emitted radiation is called fluorescence $(\mathrm{h} v \mathrm{~F})$ if it originates from an excited state of the same spin multiplicity as the ground state $\left(\mathrm{S}_{1} \rightarrow \mathrm{S}_{0}\right)$; while emission from an excited state of different spin multiplicity $\left(\mathrm{T}_{1} \rightarrow\right.$ $\left.\mathrm{S}_{0}\right)$ is called phosphorescence $(\mathrm{h} v \mathrm{P})$. Phosphorescence is of lower energy and, because it is spin forbidden, much slower (rate constants of $10^{-2} \sim 10^{4}$ $\mathrm{s}^{-1}$ ) than fluorescence (rate constants of $\left.10^{8} \sim 10^{12} \mathrm{~s}^{-1}\right)^{[79]}$. It is seldom observed because it cannot compete with the far faster processes of fluorescence, non-radiative internal conversion or bimolecular quenching by other molecules, e.g. molecular oxygen.

- Quenching (not shown) - is an important non-radiative process which competes with emission and usually involves either the transfer of electronic energy or an electron from the initially excited molecule to a quencher $(\mathrm{Q})$ via a bimolecular reaction:

$$
\mathrm{M}^{*}+\mathrm{Q} \rightarrow \mathrm{M}+\mathrm{Q}^{*}
$$


This process is also referred to as "photosensitisation", where the species, $\mathrm{Q}$, is "sensitised" by a photon of radiation absorbed by the excited $\mathrm{M}^{*}$ molecules ${ }^{[78]}$. This quenching process competes with emission as some of the $\mathrm{M}^{*}$ will react with $\mathrm{Q}$ and do not emit radiation. Unlike the intramolecular excited state transitions, this intermolecular sensitisation can cause a series of photochemical reactions and give rise to new photoproducts. A ubiquitous quencher in most environments is oxygen, and its reactions with photosensitisers lead to the production of reactive oxygen species and free radicals which are usually very damaging.

\subsubsection{Coumarins and Fluorescence of Phormium Fibres}

Molecules and other species that absorb light are called "chromophores" (and if they are fluorescent, "fluorophores") and the spectral position of their absorption in the visible region determines the colour of the material. The undyed Phormium fibres have another factor that affects colour, variations of whiteness, which is associated with the presence of strong blue fluorescent, naturally-occurring hydroxycoumarins ${ }^{[1]}$.

\section{Fluorescent Whitening Agents}

A number of substituted coumarins, with a generic chemical structure shown in Figure 3.13, emit high yields of blue fluorescence when exposed to UVA radiation $^{[80,81]}$. In fact, some synthetic 7-hydroxycoumarins have been widely used as fluorescence whitening agents (FWAs) for textiles ${ }^{[82]}$. FWAs are a class of molecules which, when excited with invisible near-UV light, give visible blueviolet fluorescence (Stokes shift) that compensates for the off-white or yellow cast of natural substrates, thus increasing the degree of 'whiteness' and 'brightness ${ }^{\text {, }}{ }^{[83]}$. Stilbenes, pyrazolines and hydroxycoumarins are the three major classes of FWAs biosynthesised in many plants as secondary metabolites. However, these compounds are also very photoreactive. They can sensitise photooxidation and 
contribute to the photodegradation of the substrate or host material ${ }^{[80,84]}$.<smiles>[R]c1c([R3])c([R])c2c([R])c([R])c(=O)oc2c1[R]</smiles>

Figure 3.13 Generic structure of coumarins $\left(\mathrm{R} 1-\mathrm{R} 6=\mathrm{H}, \mathrm{OH}, \mathrm{OCH}_{3}\right.$ or $\left.\mathrm{CH}_{3}\right)$

Among the five types of coumarins previously detected in Phormium fibres, 7-

hydroxy and methoxy coumarins that are usually encountered in higher plants ${ }^{[85,86]}$ have been suggested to contribute to the fluorescence and photodegradation ${ }^{[1]}$. Their involvement in the photodegradation of plant materials and P. tenax in particular will be covered in greater detail in Chapter 4. In order to confirm the assignment of plant fluorophores to 7-hydroxycoumarins, a comparative study of the fluorescence properties and $\mathrm{pH}$-dependent behaviours was performed on Phormium fibres, both solid fibres and aqueous extracts, with standard hydroxycoumarins by 3D-fluorescence spectroscopy.

\subsubsection{Experimental}

\section{Phormium Fibres and Preparation of Solid Samples}

Fibres of five cultivars of $P$. tenax, i.e. Arawa, Mawaru, Ngaro, Potaka and Tapamangu, were studied in this work. The presence of coumarins in these cultivars has been determined in the previous ESI-MS analysis. Solid fibre samples were prepared by compressing $0.2 \mathrm{~g}$ of finely chopped fibres into solid, self-adhering discs (13 mm diameter) with a hydraulic press. Aqueous extracts were prepared as previously described in Section 3.1.2. 


\section{7-Hydroxycoumarins}

Aqueous solutions of four 7-hydroxylated coumarins, 7-hydroxycoumarin, 4methyl-7-hydroxycoumarin, 6,7-dihydroxycoumarin and 7-hydroxy-6methoxycoumarin were used as detailed in Section 3.1.2. The $\mathrm{pH}$ measurements of solutions were carried out using a Metrohm $744 \mathrm{pH}$ meter (Herisau, Switzerland).

\section{Three Dimensional-Fluorescence Spectroscopy}

Fluorescence characterisation was performed using a Horiba Jobin-Yvon Fluorolog-3 spectrofluorometer (Edison, NJ) using the three dimensional (3D) scanning mode. 3D-fluorescence spectroscopy is a very useful technique for differentiating between different types of fluorophores present in complex, multicomponent materials often encountered in natural/biological samples.

The 3D-fluorescence spectra or excitation-emission matrices (EEMs) were obtained with excitation from 300 to $450 \mathrm{~nm}$ with increments of $10 \mathrm{~nm}$ and recorded from 320 to $600 \mathrm{~nm}$ in emission. The EEMs were then plotted using the Origin Software (Version 8.0; Microcal Inc., Northampton, MA) to display 3D fluorescence intensity contour 'maps', where the contour peaks indicate the positions of maxima in the fluorescence excitation and emission spectra of the component fluorophores.

For all fluorescence spectral characterisations reported in this thesis, discs of the solid fibre samples were fixed in a solid sample holder of the fluorometer, while aqueous extracts and solutions of hydroxycoumarins were analysed in $10 \mathrm{~mm}$ square fluorescence quartz cuvette. A "front-face" detection configuration of $22.5^{\circ}$ collection path was used in all measurements to minimize the self-absorption and attenuation of the excitation beam when working with concentrated solutions or with solid samples. 


\subsubsection{Results and Discussion}

\section{Fluorescence Spectra of Standard Hydroxycoumarins}

Figure 3.14 shows the fluorescence excitation and emission spectra of aqueous solutions of authentic samples of 7-hydroxycoumarin, 4-methyl-7hydroxycoumarin, 6,7-dihydroxycoumarin and 7-hydroxy-6-methoxycoumarin obtained at different pHs. Of the panels of twelve contour maps, the middle column represents the neutral ( $\mathrm{pHs} 6.0$ - 7.6) extracted solutions, with the acid/alkaline $\mathrm{pHs}$ on either side were adjusted by the addition of dilute hydrochloric acid or sodium hydroxide solutions, respectively (rather than buffering all solutions at the same $\mathrm{pH}$, this method gave rise to a range of acid/alkaline pHs as shown in each column). For each hydroxycoumarin, a set of three EMMs were plotted with 10 intensity contour lines, where every contour interval represents 0.1 of the maximum fluorescence intensity from the sample with the greatest fluorescence yield. In some plots such as A and C in the first two columns, a set of small peaks that occur diagonally with the analyte fluorescence can be seen, which is due to the Raman scatter artifact of the water solvent.

The $\mathrm{pK}_{\mathrm{a}}$ values for ground state of 7-hydroxycoumarin, 4-methyl-7hydroxycoumarin, 6,7-dihydroxycoumarin and 7-hydroxy-6-methoxycoumarin are 7.8, 7.9, 6.8 and 7.6 respectively ${ }^{[87,88]}$. Therefore, the $\mathrm{pH}$-dependent peak-shift in fluorescence spectra can be explained in terms of absorption/excitation of the ground state of the anionic forms of the hydroxycoumarins at $\mathrm{pH}>8$ to the neutral and/or protonated forms at $\mathrm{pH}<8$ as discussed below.

In the alkaline solutions, almost all molecules exist as anions (except the two merged peaks in 4-methyl-7-hydroxycoumarin which may correspond to a coincidence of both neutral and anion molecules). Below $\mathrm{pH} 8$, the excitation maxima shift to shorter wavelengths, as a result of the protonation of the phenolic hydroxyl groups. However, the fluorescence emissions remain that of the deprotonated phenolate ions (around $450-460 \mathrm{~nm}$ ) whether excited from the 
ground state protonated or anionic deprotonated form. This is attributed to a rapid deprotonation in the first excited state ${ }^{[88]}$ which is very much more acidic than the ground state. A significant reduction in the intensity was observed in acidic solutions of 6,7-dihydroxycoumarin and 7-hydroxy-6-methoxycoumarin.

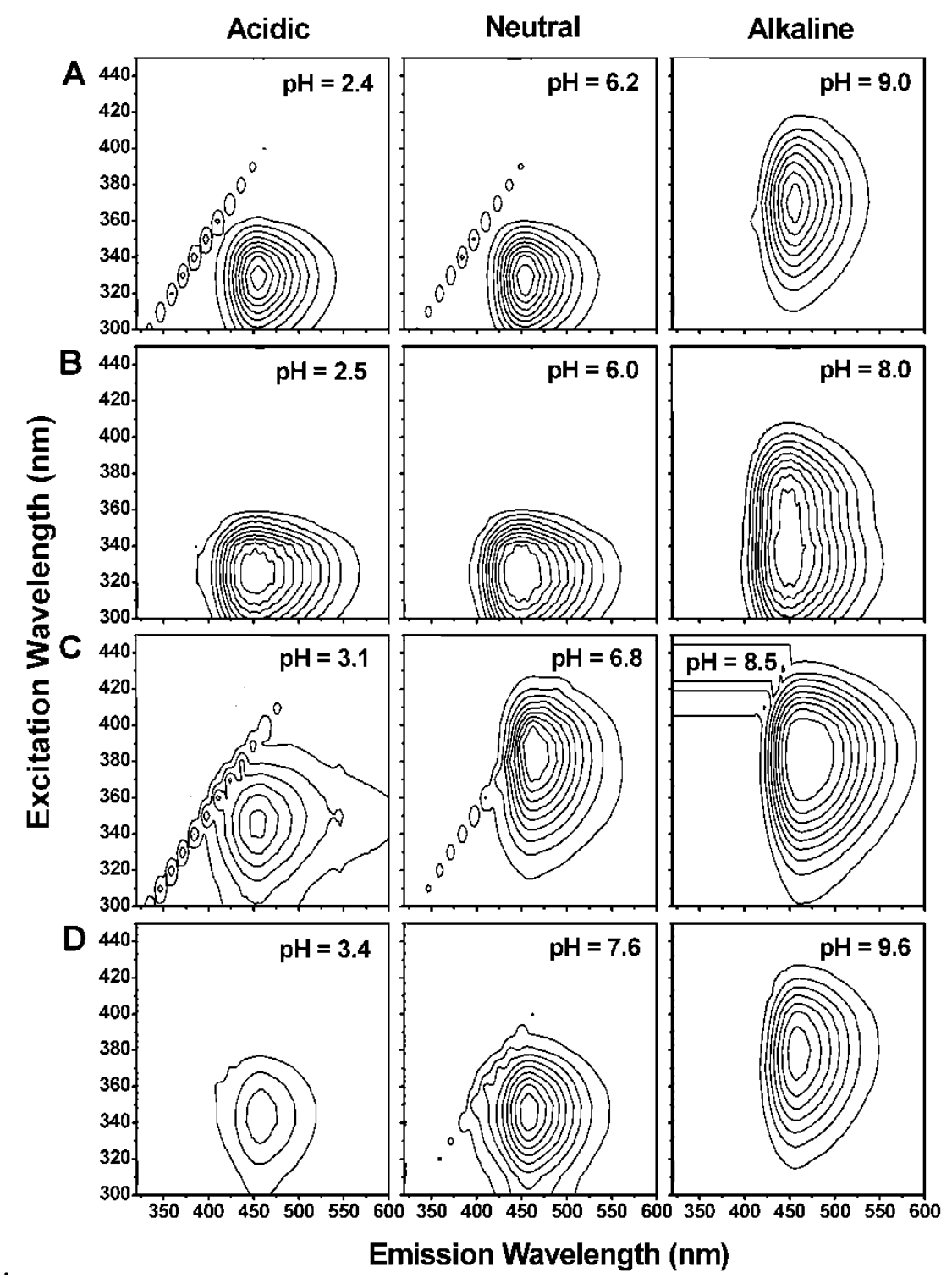

Figure 3.14 3D-fluorescence spectra of aqueous solutions of four 7hydroxycoumarins: 7-hydroxycoumarin (A), 4-methyl-7-hydroxycoumarin (B), 6,7dihydroxycoumarin (C) and 7-hydroxy-6-methoxycoumarin (D) at different pHs from acidic to alkaline. Contour intervals represent 1/10th of the maximum fluorescence of the three plots of a sample. 


\section{Fluorescence Spectra of Phormium Fibres and the Aqueous}

\section{Extracts}

The 3D-fluorescence spectra of the five cultivars of Phormium fibres are shown in Figure 3.15. The contour plots are in arbitrary units (20 contour intervals of the maximum fluorescence). All cultivars exhibited fluorescence emission in the blue region with a maximum around $450 \mathrm{~nm}$ and excitation maxima at wavelengths from $350 \mathrm{~nm}$ to $370 \mathrm{~nm}$, which are consistent with the fluorescence excitation/emission wavelengths of authentic samples of hydroxycoumarins shown in Figure 3.14.

Aqueous extracts of Phormium fibres exhibited similar fluorescence emission under near-neutral and acidic conditions as shown in Figure 3.16. The excitation maxima of different cultivars vary in the range of 320 to $380 \mathrm{~nm}$. Similar $\mathrm{pH}$ dependency was also observed. When the $\mathrm{pH}$ is increased to 8.5 , the contour positions shifted to longer excitation wavelengths, together with the increases in the fluorescence intensity.

Also of interest to note is the trend in the fluorescence intensity of the five cultivars, as follows: Arawa $>$ Tapamangu $>$ Ngaro $>$ Mawaru $>$ Potaka in both solid and aqueous samples. As discussed earlier, the fluorescence emission is dependent on the state of protonation of phenolic hydroxyl groups. The fluorescence of Phormium fibres can therefore be attributed to a mixture of deprotonated forms of hydroxy- and/or hydroxymethoxycoumarins. The mass spectral data and fluorescence spectra of standard compounds support the assignment of fibre fluorophores, at least in part, to the presence of naturallyoccurring 7-hydroxycoumarins. Additionally, their varying fluorescence intensities could have contributed to the natural variations of whiteness observed for different cultivars. 


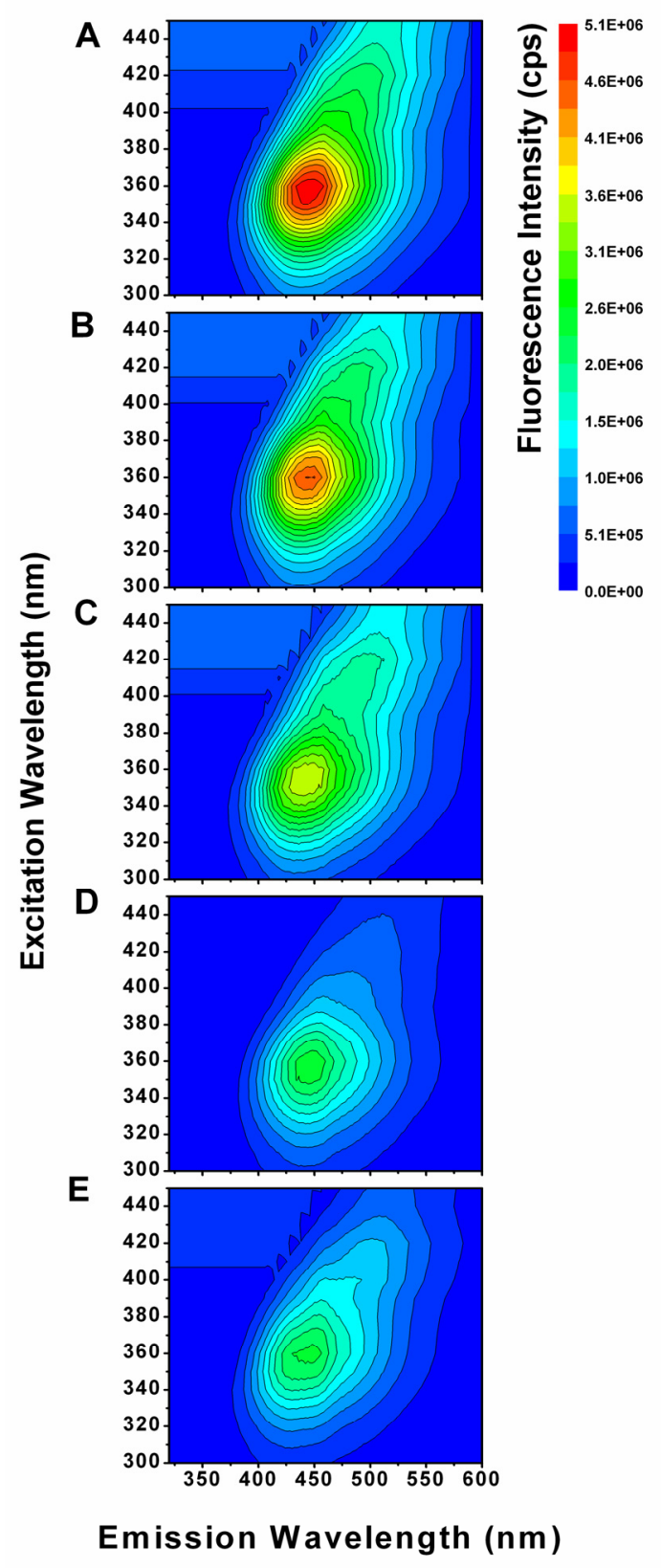

Figure 3.15 3D-fluorescence spectra of solid Phormium fibres of five cultivars: Arawa (A), Ngaro (B), Tapamangu (C), Mawaru (D) and Potaka (E). 


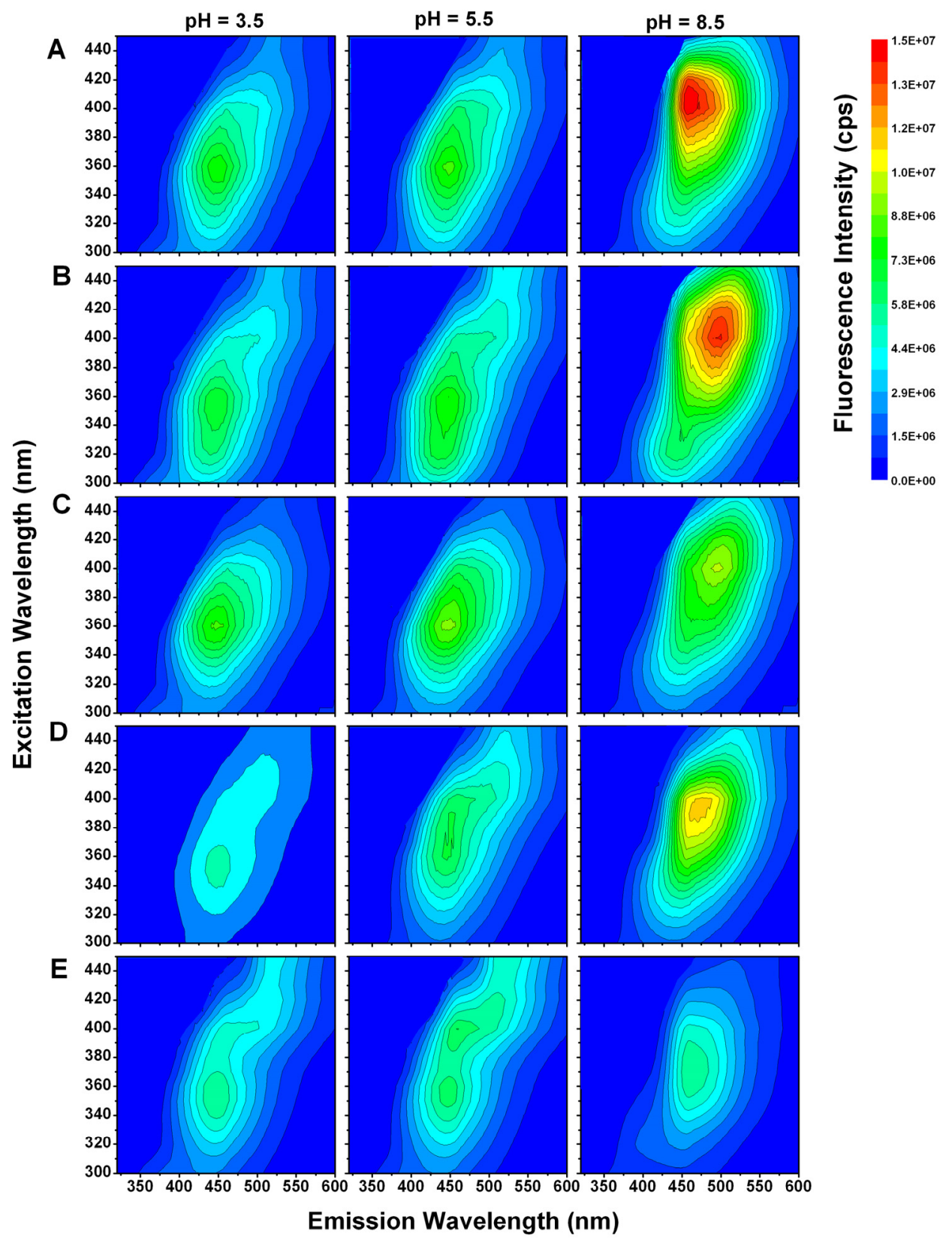

Figure 3.16 3D-fluorescence spectra of aqueous extracts from Phormium fibres of five cultivars: Arawa (A), Tapamangu (B), Ngaro (C), Mawaru (D) and Potaka (E), under acidic, neutral and alkaline conditions. 


\subsection{Summary of the Chapter}

Electrospray ionisation tandem mass spectrometry (ESI-MS/MS) is a rapid and sensitive characterisation technique using "soft" ionisation that is especially suited for trace, fragile biomolecules from complex mixtures in solution. Five compounds that have antifungal activities, viz. furfural, coumarin, 4-methyl-7hydroxycoumarin, 7-hydroxy-6-methoxycoumarin 6,7-dihydroxycoumarin and 7hydroxycoumarin, were identified in the aqueous extracts from the fibres of six cultivars of $P$. tenax by ESI-MS. The numbers of compounds identified in the tested cultivars could be correlated to their varying antifungal efficacies displayed in the in vitro mycological screening (see Chapter 2) which could have implicated a synergistic effect of these antifungal agents.

In addition to their role as plant antifungal metabolites, many substituted coumarins are known to emit strong fluorescence in the violet-blue region and can be applied as fluorescent whitening agents to various substrates to enhance their "whiteness" and "brightness". 3D-fluorescence spectrometry was utilised for quantitative evaluation of fluorescence intensity and characterisation of their emission/excitation spectra. Phormium fibres from five of the six cultivars exhibited similar fluorescence emissions to those originating from the four 7hydroxycoumarins. This, together with the matching fluorescence $\mathrm{pH}$-dependent behaviours with authentic compounds, justifies the assignment of fibre fluorescence, at least in part, to the deprotonated forms of a number of naturally occurring 7-hydroxycoumarins present in the fibre. Considerable differences between the hydroxycoumarin contents and compositions of the various cultivars studied in this thesis were also observed. 


\title{
Chapter 4
}

\section{Photodegradation of Phormium Fibres: Coumarin Photochemistry}

\begin{abstract}
Absorption of ultraviolet (UV) wavelengths $<400 \mathrm{~nm}$ and visible light in the range of $400-700 \mathrm{~nm}$, by molecules results in excitation of electrons from their highest occupied molecular orbitals (HOMO) of the ground state to unoccupied molecular orbitals in the excited state. The energies or wavelengths of the photons absorbed depend on the energies of the HOMO and the excited state energy levels. These electronic excited states are much more reactive than the ground states and can undergo a number of physical processes and/or chemical reactions. This chapter describes the photoreactions of Phormium fibres of five cultivars and the hydroxycoumarins present in the fibre upon exposure to UVA radiation.
\end{abstract}

\subsection{Introduction to Photodegradation}

\section{Photodegradation of Lignocellulosic Materials}

Photodegradation of lignocellulosic materials when they are exposed to sunlight, particularly in the UV region, is manifested as discolouration (photoyellowing), loss of tensile strength and elasticity (phototendering) ${ }^{[89,90]}$. Many photochemical reactions including oxidation, hydrolysis, free radical cross-linking and polymerisation are involved, and various other factors such as wavelength, 
temperature, humidity, the presence of oxygen and chemical agents can influence this $\operatorname{process}^{[90,91]}$.

Pure celluloses such as cotton are poor absorbers of solar UV or visible light, whereas the lignin present in many plant fibres absorbs strongly over a broadband of radiation wavelengths from sunlight and is thereby more likely to contribute to the photodegradation of natural lignocellulosic materials ${ }^{[90]}$. Studies on the photochemical behaviours of model compounds have revealed some important information of the photodegradation mechanisms. For example, phototendering has been found to mainly result from the fragmentation of cellulose molecules upon UV irradiation of wavelengths shorter than $300 \mathrm{~nm}$ as well as the associated weight loss due to the formation of volatile photoproducts $^{[92]}$. Photoyellowing has been shown to involve photooxidation. Although numerous studies have been devoted to this subject, the mechanism is far from understood ${ }^{[93]}$. Among the many photoreactions that contribute to photoyellowing of lignin, the ring-conjugated carbonyl groups of lignin have been considered as an important contributing structural motif to the formation of coloured chromophores ${ }^{[94,95]}$. These photoreactive groups of lignin are capable of abstracting hydrogen atoms from phenolic groups to generate phenoxy radicals to form ketones and/or conjugated quinone species giving colouration to the irradiated materials ${ }^{[90,93]}$.

In many of these photodegradation processes, the participation of molecular oxygen is implied, and the main degradative reaction is considered to be a photosensitised oxidation via the formation of activated reactive oxygen species (ROS). Incorporation of external sensitisers, such as FWAs ${ }^{[96,97]}$, some polyphenolic dyes ${ }^{[85]}$ and metal ions (e.g. $\mathrm{Fe}^{2+}$ and $\left.\mathrm{Cu}^{2+}\right)^{[89]}$ can further increase the photosensitivity of the system and thereby significantly increase the rate of photodegradation. An increase in the moisture content of substrates has also been found to accelerate the process ${ }^{[91]}$. General mechanisms of photosensitised formation of ROS are given below. 


\section{General Mechanisms of Oxygen Photoactivation}

Derived from molecular oxygen, ROS include singlet oxygen, hydrogen peroxide and reactive radicals. A number of these reactive species occur in biological systems as stress signalling molecules in regulating important physiological processes $^{[98]}$. However in the context of this thesis, their photolytic formation is the significant issue as precursors for photodegradation. When exposed to UV radiation, many photosensitisers $\left({ }^{1} \mathrm{P}\right)$ produce excited singlet $\left({ }^{1} \mathrm{P}^{*}\right)$ and triplet $\left({ }^{3} \mathrm{P}^{*}\right)$ states, which can give rise to reactive radicals and activate ROS by one of the two principal mechanisms shown in Figure 4.1.

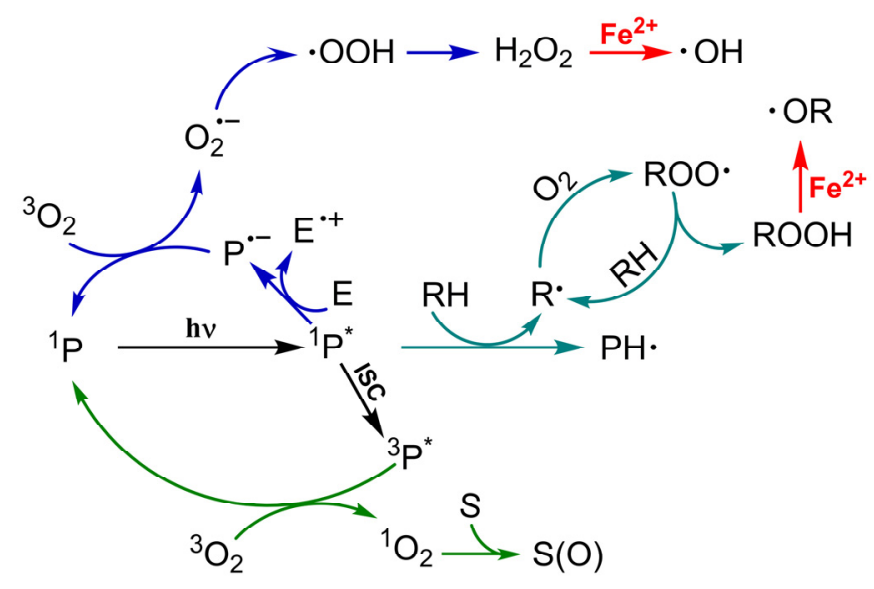

Figure 4.1 Two major mechanisms of photosensitised formation of ROS: type I (cyan for the hydrogen transfer scheme; blue for the electron transfer scheme) and type II (green). Fenton reactions are marked in red ${ }^{[99]}$.

- Atmospheric oxygen in the triplet ground state $\left({ }^{3} \mathrm{O}_{2}\right)$ can undergo photosensitised spin inversion to form singlet oxygen $\left({ }^{1} \mathrm{O}_{2}\right)$ by a Type II mechanism (shown as green arrows in the figure) or direct excitation energy transfer between ${ }^{3} \mathrm{P}^{*}$ and ${ }^{3} \mathrm{O}_{2}$ :

$$
{ }^{3} \mathrm{P}^{*}+{ }^{3} \mathrm{O}_{2} \rightarrow{ }^{1} \mathrm{P}+{ }^{1} \mathrm{O}_{2}
$$


${ }^{1} \mathrm{O}_{2}$ reacts rapidly with many substrates (S) containing olefins, giving oxidation products:

$$
{ }^{1} \mathrm{O}_{2}+\mathrm{S} \rightarrow \mathrm{S}(\mathrm{O})
$$

- Reactions involving the excited singlet states of a photosensitiser $\left({ }^{1} \mathrm{P}^{*}\right)$ with another molecule to transfer an electron or a hydrogen atom are classified as Type I, and indicated by blue or cyan arrows in the figure. In the presence of an electron donor (E), molecular oxygen can be reduced with one electron to yield superoxide anion $\left(\mathrm{O}_{2}{ }^{-}\right)$. At high concentrations, the photosensitiser, $\mathrm{P}$, itself can sometimes act as the electron donor, producing semi-reduced and semi-oxidised radicals ${ }^{[97]}$ :

$$
\begin{gathered}
\mathrm{P}^{*}+\mathrm{E} \rightarrow \mathrm{P}^{\cdot-}+\mathrm{E}^{\cdot+} \\
\mathrm{P}^{\cdot-}+\mathrm{O}_{2} \rightarrow \mathrm{P}+\mathrm{O}_{2}^{\cdot-} \\
\mathrm{P}^{*}+\mathrm{O}_{2} \rightarrow \mathrm{P}^{\cdot+}+\mathrm{O}_{2}^{\cdot-}
\end{gathered}
$$

$\mathrm{O}_{2}{ }^{--}$can react in aqueous environment to produce hydrogen peroxide $\left(\mathrm{H}_{2} \mathrm{O}_{2}\right)$ via the formation of hydroperoxy free radicals $(\mathrm{HOO})^{[100]}$ :

$$
\begin{gathered}
\mathrm{O}_{2}^{--}+\mathrm{H}_{2} \mathrm{O} \rightarrow \mathrm{HOO}^{\bullet}+\mathrm{OH}^{-} \\
2 \mathrm{HOO}^{\cdot} \rightarrow \mathrm{H}_{2} \mathrm{O}_{2}+\mathrm{O}_{2}
\end{gathered}
$$

$\mathrm{H}_{2} \mathrm{O}_{2}$ can be monovalently reduced by transition metal ions, such as $\mathrm{Fe}^{2+}$, yielding the extremely reactive hydroxyl radical $\left(\mathrm{OH}^{*}\right)$. This is also known as the "Fenton Reaction" (marked in red in the figure) ${ }^{[101]}$.

$$
\mathrm{Fe}^{2+}+\mathrm{H}_{2} \mathrm{O}_{2} \rightarrow \mathrm{Fe}^{3+}+\mathrm{OH}^{\bullet}+\mathrm{OH}^{-}
$$

- The second Type I process (cyan arrows) involves the transfer of a hydrogen atom (reduction) from an organic molecule $(\mathrm{RH})$ to the photoexcited $\mathrm{P}^{*}$. This generates free radicals capable of rapidly reacting 
with molecular oxygen and creating a complex mixture of reactive oxygen intermediates, including reactive peroxides:

$$
\begin{gathered}
\mathrm{P}^{*}+\mathrm{RH} \rightarrow \mathrm{PH}^{\bullet}+\mathrm{R}^{\bullet} \\
\mathrm{R}^{\bullet}+\mathrm{O}_{2} \rightarrow \mathrm{ROO}^{\bullet} \\
\mathrm{ROO}^{\bullet}+\mathrm{RH} \rightarrow \mathrm{ROOH}+\mathrm{R}^{\bullet}
\end{gathered}
$$

By analogy with reaction 4.8, $\mathrm{ROOH}$ can be reduced by one-electron donors such as $\mathrm{Fe}^{2+}$ to yield alkoxyl radicals ${ }^{[99]}$ :

$$
\mathrm{Fe}^{2+}+\mathrm{ROOH} \rightarrow \mathrm{Fe}^{3+}+\mathrm{OR}^{\cdot}+\mathrm{OH}^{-}
$$

The superoxide ion, peroxides, singlet oxygen and free radical products of photosensitisation reactions may react further by oxidising other molecules and/or initiating chain reactions, leading to significant photooxidation and photodegradation of lignocellulosic materials.

\section{Coumarins and Photoyellowing of Phormium Fibres}

Like other lignocellulosic materials, Phormium fibres are subject to yellowing when exposed to near-UV radiation ${ }^{[22]}$. This fibre type has been reported to have lignin compositions ranging from $11 \%$ to $72 \%$, depending on the plant cultivar ${ }^{[3]}$. Although details of the chemistry of the photoyellowing process are not fully elucidated, light-induced reactions of lignin and/or its precursors in the fibre are purported to be important, and the naturally-occurring coumarins identified for the first time in this project are suspected to initiate and/or participate in the photochemical degradation of Phormium fibres.

As important secondary metabolites, coumarins are ubiquitously produced in higher plants and have been detected in many lignocellulosic materials ${ }^{[1,60,102]}$. The biosynthesis of these compounds can be induced as a phytoalexin upon mechanical wounding or abiotic stresses on living plants, and many of these coumarins possess antifungal (See Chapter 2) and antibiotic activities ${ }^{[60,103]}$. 
Coumarins are also present as constitutive secondary metabolites in many lignocellulosic fibres. Genetic studies using Arabidopsis mutants have indicated that coumarin and its hydroxylated derivatives originate mainly from the phenylpropanoid pathway which is also responsible for the biosynthesis of lignin and many phenolic metabolites ${ }^{[104-106]}$. Several participating genes and enzymes, particularly the p-coumaroyl-shikimate/quinate - 3'- hydroxylase, have been shown to be essential to this biosynthetic machinery ${ }^{[107]}$.

The photochemical reactions of coumarins, initiated by UV-irradiation, such as photodimerisation, photooxidation and photosensitised electron-transfer reactions, have been investigated ${ }^{[80,108-110]}$. The photodimerisation of coumarins has been intensively studied previously. The distribution of coumarin photodimers in solution was found to be dependent on solvent polarity and the spin multiplicity of the excited states ${ }^{[109-111]}$. Further, when coumarin groups are attached to polymers, they were able to photo-crosslink the polymers through the coumarin moiety to form insoluble networks where the light sensitivity of the polymer was directly proportional to the degree of coumarin substitution ${ }^{[80]}$. Photodimerisation of solidstate coumarin and derivatives, both in the bulk ${ }^{[112]}$ and in inclusion complexes ${ }^{[113]}$, has been reported to be affected by molecular packing and some substituents. The observation of a complete inertness of coumarin and hydroxycoumarins in the solid state has suggested a different photodegradation pathway. Photooxidation of some coumarin dyes, together with measurements of the sensitised formation of ROS, have also been investigated $\left.{ }^{[114,} 115\right]$. However, reports concerning the photochemistry of coumarins in aqueous solution are comparatively few ${ }^{[80,116]}$.

In order to assess the role of the naturally-occurring coumarins, especially 7hydroxycoumarins, in the photoyellowing of Phormium fibres, the photochemical properties of Phormium fibres and two 7-hydroxycoumarins identifed in the fibre were investigated by UV-Vis/fluorescence spectroscopy and by measurement of ROS yields upon UVA-irradiation. An ESI-MS/MS analysis of the photoproducts formed by photolysis of the two standard coumarins in water was also carried out to further understand the photodegradation of Phormium fibres. 


\subsection{Materials and Methods}

\subsubsection{Materials}

\section{7-Hydroxycoumarins}

Unless otherwise stated, chemicals were purchased from Sigma-Aldrich. The two standard 7-hydroxycoumarins studied are: 6,7-dihydroxycoumarin (esculetin, $98 \%$ ) and 7-hydroxy-6-methoxycoumarin (scopoletin, $99 \%$ ). Saturated aqueous solutions of both coumarins were prepared in double distilled water (DDW) without further purification.

\section{Phormium Fibres}

Phormium fibres were extracted from the leaves of five cultivars of P. tenax: Arawa, Mawaru, Ngaro, Potaka and Tapamangu. These fibres contained a number of naturally-occurring coumarins that were identified by ESI-MS/MS (see Chapter 3). Portions of the fibres weighing $0.2 \mathrm{~g}$ were finely chopped and pressed into solid, self-adhering discs with a diameter of $13 \mathrm{~mm}$ using a hydraulic press. These discs were used for the fluorescence and reflectance spectroscopic measurements.

\subsubsection{Irradiation of Samples}

The photoirradiation was performed using two $6 \mathrm{~W}$ 'blacklight' UV fluorescent lamps (NEC FL6BL-B) with the spectral distribution $\left(\lambda_{\max }=366 \mathrm{~nm}\right)$ shown in Figure 4.2. The lamps were cooled by a small fan beneath the sample, so that the temperature can be kept between $20{ }^{\circ} \mathrm{C}$ and $25{ }^{\circ} \mathrm{C}$ even during extended (up to 96 hrs) irradiation periods. The absolute spectral irradiance of the lamps was determined from 320 to $400 \mathrm{~nm}$ (UVA) at $1 \mathrm{~nm}$ intervals using a 
spectroradiometer based on a double monochromator (GM301, Kratos-Schoeffel, NJ, USA), which was calibrated by the Measurement Standards Laboratory of New Zealand. The integrated intensity of irradiance incident on a sample positioned at a distance of $1 \mathrm{~cm}$ was $4.2 \mathrm{~mW} \cdot \mathrm{cm}^{-2}$.

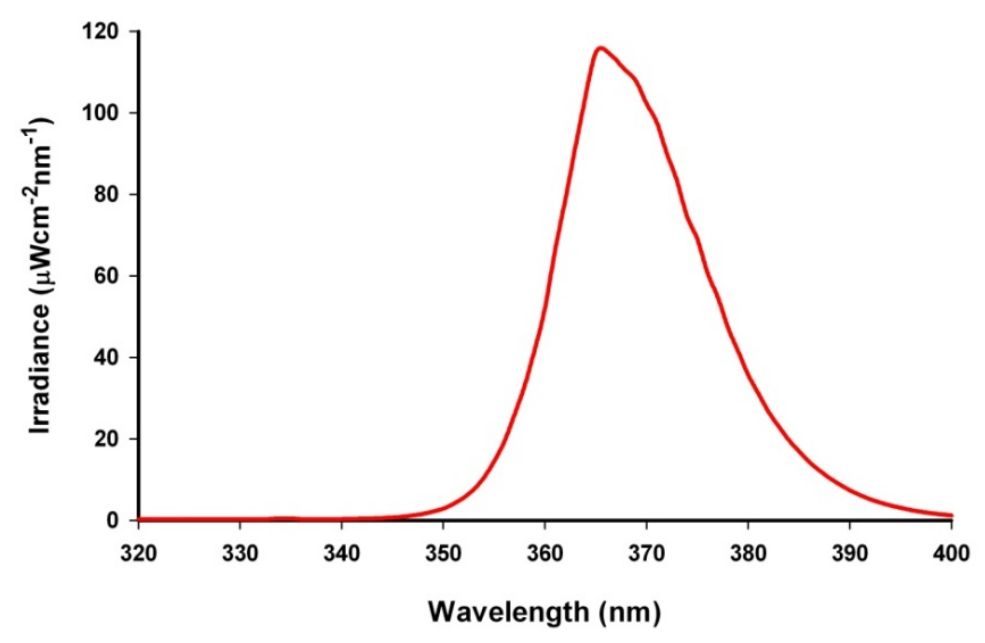

Figure 4.2 The absolute spectral distribution of UVA irradiance on a sample positioned $1 \mathrm{~cm}$ away from a $6 \mathrm{~W}$, NEC 'blacklight' fluorescent UV lamp.

For irradiation under wet conditions, $0.2 \mathrm{~g}$ of fibre samples were moistened with 2 $\mathrm{mL}$ of DDW and hermetically sealed in a polyethylene container with an optical transmission of $75-80 \%$ over the spectral range of the irradiating source from 350 to $400 \mathrm{~nm}$. After irradiation, an aliquot of the water in contact with the fibre samples was removed for ROS assays (see Chapter 5). The solids were dried in ambient air before they were repressed into discs for fluorescence and reflectance spectroscopic measurements. Saturated aqueous solutions of standard coumarins $(2 \mathrm{~mL})$ were irradiated in quartz cuvettes. 


\subsubsection{Spectroscopic Characterisations}

\section{UV/Vis-Absorption Spectroscopy}

The absorption spectra of aqueous solutions of 7-hydroxycoumarins were recorded using a $2 \mathrm{~mm}$ path-length quartz cuvette and a double-beam Cary 100 UV/Vis spectrophotometer (Varian, Palo Alto, CA).

\section{Reflectance Spectroscopy}

Diffuse reflectance spectra of solid fibre samples in the form of $13 \mathrm{~mm}$-diameter compressed discs, before and after UVA irradiation, were recorded using the same Cary $100 \mathrm{UV} /$ Vis spectrophotometer with an integrating sphere attachment.

\section{D-Fluorescence Spectroscopy}

3D-Fluorescence measurements were performed using a Horiba Jobin-Yvon Fluorolog-3 spectrofluorometer (Edison, NJ) with a "front-face" detection configuration. Compressed discs of $0.2 \mathrm{~g}$ of solid samples were fixed in a sample holder of the fluorometer, while aqueous samples were run in quartz cuvettes. The method for data processing was described in Chapter 3, Section 3.2.

\section{ESI-Mass Spectrometry}

ESI-mass spectrometric analysis of 6,7-dihydroxycoumarin after $0 \mathrm{~h}$ to $24 \mathrm{hrs}$ of UVA-irradiation was performed using a tandem quadrupole time-of-flight (QqTOF) mass spectrometer (QSTAR Pulsar-I, Applied Biosystems, Foster City, CA), with perfusion nanospray delivery of aqueous samples. Analysis of UVAirradiated 7-hydroxy-6-methoxycoumarin was performed on a Waters Micromass Qq-TOF Premier mass spectrometer (Waters, Manchester, UK). 


\subsubsection{Superoxide and Peroxide Assays}

\section{Chemical Reagents}

Sorbitol ((2S,3R,4R,5R)-hexane-1,2,3,4,5,6-hexol), xylenol orange (3,3'-bis[N,Ndi(carboxymethyl)-aminomethyl]-o-cresolsulfone-phthalein, tetrasodium salt), and two enzymes, catalase (bovine liver) and superoxide dismutase (SOD) were purchased from Sigma-Aldrich. Hydrogen peroxide standards were prepared by dilution with double distilled water (DDW) from an AnalaR stock solution (30\% w/v). Ammonium iron (II) sulphate 6-hydrate was supplied by Purescience (Wellington, NZ).

\section{Hydrogen Peroxide from Irradiated Coumarin Solution}

The quantity of hydrogen peroxide $\left(\mathrm{H}_{2} \mathrm{O}_{2}\right)$ produced by UVA irradiated samples was measured using a colorimetric method of Jiang ${ }^{[117]}$ and Millington ${ }^{[97]}$. The assay was based on the oxidation of iron (II) to iron (III) by $\mathrm{H}_{2} \mathrm{O}_{2}$ under acidic conditions. Sorbitol acts as a catalyst in this process. Iron (III) then complexes with an indicator dye, xylenol orange (XO), to form a purple chromophore with an absorption maximum at $560 \mathrm{~nm}$.

$$
\begin{gathered}
\mathrm{Fe}^{2+}+\mathrm{H}_{2} \mathrm{O}_{2} \rightarrow \mathrm{Fe}^{3+}+\mathrm{HO}^{\cdot}+\mathrm{OH}^{-} \\
\mathrm{Fe}^{3+}+\mathrm{XO} \rightarrow \mathrm{Fe}^{3+}-\mathrm{XO}
\end{gathered}
$$

The assay was carried out in aqueous solution, in which a $5 \mathrm{~mL}$ aliquot of irradiated solution or peroxide standard was added to $10 \mathrm{~mL}$ of working reagent solution, with DDW making up a total volume of $25 \mathrm{~mL}$. The working reagent was prepared fresh daily by mixing equal aliquots of sulphuric acid $(0.25 \mathrm{M})$, sorbitol $(0.1 \mathrm{M})$, xylenol orange $(1.0 \mathrm{mM})$ and ammonium iron (II) sulphate (2.5 $\mathrm{mM})$. The solution was held at room temperature with mild agitation for $45 \mathrm{~min}$ before determining the absorbance at $560 \mathrm{~nm}$ by a double-beam UV-Vis 
spectrophotometer (Cary 100 Scan, Varian).

A set of peroxide standards covering a range of known concentrations was prepared by diluting from a $25 \mathrm{mM} \mathrm{H}_{2} \mathrm{O}_{2}$ stock solution that had been standardised using the molar extinction coefficient $(\varepsilon)$ of $43.6 \mathrm{M}^{-1} \cdot \mathrm{cm}^{-1}$ at $240 \mathrm{~nm}$. A standard curve of $\mathrm{H}_{2} \mathrm{O}_{2}$ can therefore be plotted out by linear regression as shown in Figure 4.3, in which the obtained value of extinction coefficient ( $\varepsilon=$ $2.67 \times 10^{5} \mathrm{M} \cdot \mathrm{cm}^{-1}$ at $560 \mathrm{~nm}$ ) was in good agreement with literature ${ }^{[117]}$. The concentration of $\mathrm{H}_{2} \mathrm{O}_{2}$ can therefore be read graphically from this standard curve or be calculated out using the first-degree function as fitted in the figure.

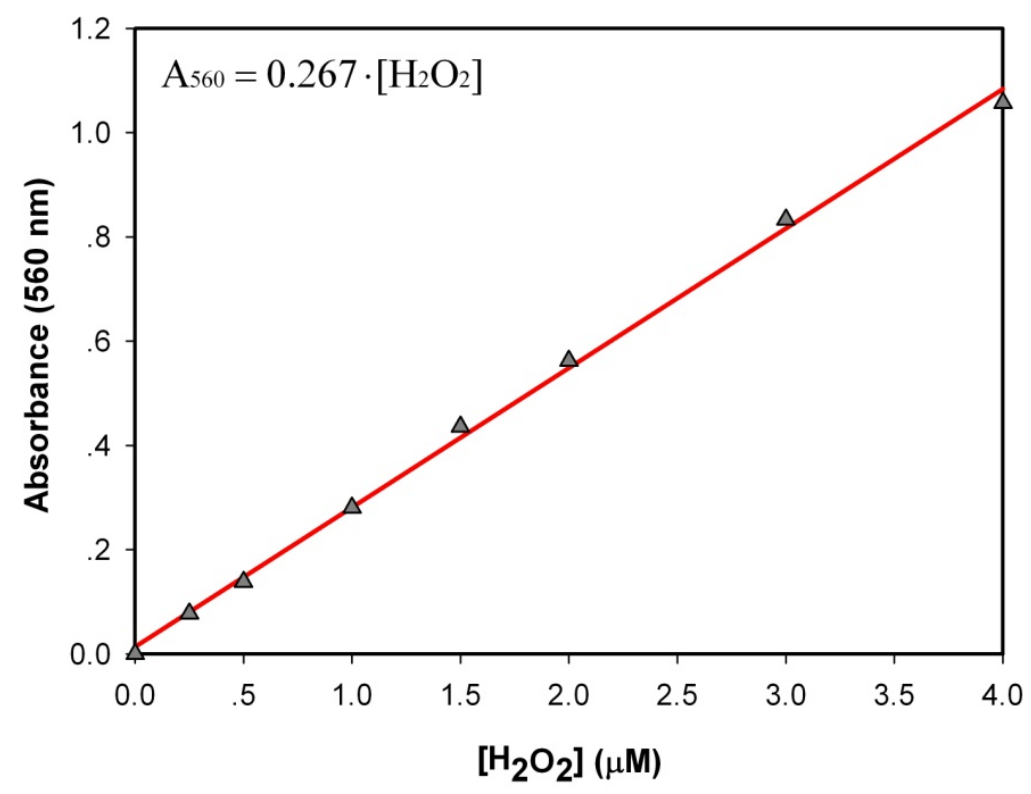

Figure 4.3 Standard curve of $\left[\mathrm{H}_{2} \mathrm{O}_{2}\right]$ in the range of 0 to $4.0 \mu \mathrm{M}$. Data were the mean of triplicate determinations.

\section{Hydrogen Peroxide from Irradiated Fibre}

After irradiation, a $0.5 \mathrm{~mL}$ aliquot of the water that had been in contact with the solid fibres was obtained by centrifugation at $14,000 \mathrm{rps}$ for $10 \mathrm{~min}$. The liquid was further diluted with DDW to make a final total volume of $5 \mathrm{~mL}$. The xylenol orange peroxide assay was then performed on this $5 \mathrm{~mL}$ analyte, and the yield of 
$\mathrm{H}_{2} \mathrm{O}_{2}$ was determined by reference to the standard curve (Figure 4.3). An aliquot of unirradiated sample was used as the blank. The assay for each fibre cultivar was carried out in triplicate on both irradiated samples and the dark control. In order to obtain improved accuracy, an identical experiment in the absence of peroxide was also performed, in which catalase (250 Units $\left.\cdot \mathrm{mL}^{-1}\right)$, a highly specific enzyme for $\mathrm{H}_{2} \mathrm{O}_{2}$, was added in the reaction mixture. Since any $\mathrm{H}_{2} \mathrm{O}_{2}$ would be selectively converted to water and oxygen (Reaction 4.15), the presence of any ferric ions in the plant extract or other oxidants capable of producing iron (III) could thereby be established and subtracted.

$$
2 \mathrm{H}_{2} \mathrm{O}_{2} \stackrel{\text { catalase }}{\longrightarrow} \mathrm{O}_{2}+2 \mathrm{H}_{2} \mathrm{O}
$$

\section{Superoxide Radicals}

The quantities of superoxide produced in irradiated samples were determined in the same reaction system with an addition of superoxide dismutase (SOD) ${ }^{[97]}$, a highly efficient enzyme that quantitatively converts superoxide radical anions $\left.\left(\mathrm{O}_{2}{ }^{-}\right)^{-}\right)$and the protonated form $\mathrm{HOO}^{\cdot[118,119]}$ to $\mathrm{H}_{2} \mathrm{O}_{2}$ (Reaction 4.16). The amount of superoxide produced is therefore deduced by an enhancement of $\mathrm{H}_{2} \mathrm{O}_{2}$ production. To avoid enzyme inactivation by the UVA radiation, catalase and SOD were added dropwise to the reaction system $\left(250 \mathrm{Units} \cdot \mathrm{mL}^{-1}\right)$ after irradiation and prior to the colorimetric assay.

$$
\mathrm{O}_{2}^{--}+2 \mathrm{H}_{2} \mathrm{O} \stackrel{\text { SOD }}{\longrightarrow} 2 \mathrm{H}_{2} \mathrm{O}_{2}
$$




\subsection{Results and Discussion}

\subsubsection{Optical Effects of UVA-Radiation}

\section{Phormium Fibres of Five Cultivars}

The photochemical responses of Phormium fibres of the five cultivars, in the presence and absence of water, after 24 hrs of UVA irradiation were studied. The $\mathrm{UV}-\mathrm{Vis}$ reflectance and 3D-fluorescence spectra for fibres before and after irradiation are given in Figure 4.4 and Figure 4.5, respectively. The results are summarised below:

- After irradiation, the reflectance values of the fibres decreased over the entire visible spectrum (Figure 4.4), indicating the formation of a number of products absorbing across this spectral range. For some cultivars, more pronounced changes were observed when the fibres were irradiated under wet conditions than in the dry state. This was most pronounced for Arawa and Ngaro, which displayed substantial reduction of reflectance in the UV to blue spectral region, wavelengths $350-430 \mathrm{~nm}$, when irradiated in the presence of water.

- In the 3D-fluorescence spectra (Figure 4.5), exposure of fibres to UVA radiation resulted in substantial losses of the coumarin-like fluorophores (as specified in Chapter 3, Section 3.3) making the fibres appear duller and yellower. However, fluorescent species which emitted at longer wavelengths towards $470 \mathrm{~nm}$ were also produced. This emission may have slightly offset the decrease in brightness resulting from the degradation of the major fluorophores. Again, irradiations in the wet state were found to result in a more significant decrease of the fluorescence intensity, which is consistent with the observed reduction in the reflectance spectra. 
- In both reflectance and fluorescence spectra, there are discernable intercultivar differences. Firstly, the five Phormium cultivars studied in this work differed considerably in the yellowing rates as indicated by reductions in their blue reflectance. Secondly, in the fluorescence spectrum of wet-irradiated fibres of cultivar Arawa, there was a formation of new fluorophores with an excitation maximum at $450 \mathrm{~nm}$ and an emission maximum at $520 \mathrm{~nm}$, which was not observed in other cultivars and is thereby indicative of the formation of new fluorescent photoproducts in an aqueous environment. However, in the case of cultivar Potaka, as well as a larger decrease of emission yields, the excitation and emission spectral changes observed for the wet-irradiated sample are less than those obtained from the dry sample. The other three cultivars exhibited a gradual decrease in fluorescence emission and a moderate shift in fluorophores, excitation and emission, to longer wavelengths.

- As a result of the yellowing-related reductions of reflectance and of fluorescence intensity, the relative photoyellowing of the Phormium cultivars examined can be ranked in the order: Arawa > Ngaro > Tapamangu $>$ Mawaru $>$ Potaka. Interestingly, this order of photosensitivity of the five cultivars studied is in accordance with their relative antifungal activities demonstrated in the solid fibre mycological screening (see Chapter 2, Section 2.2). 


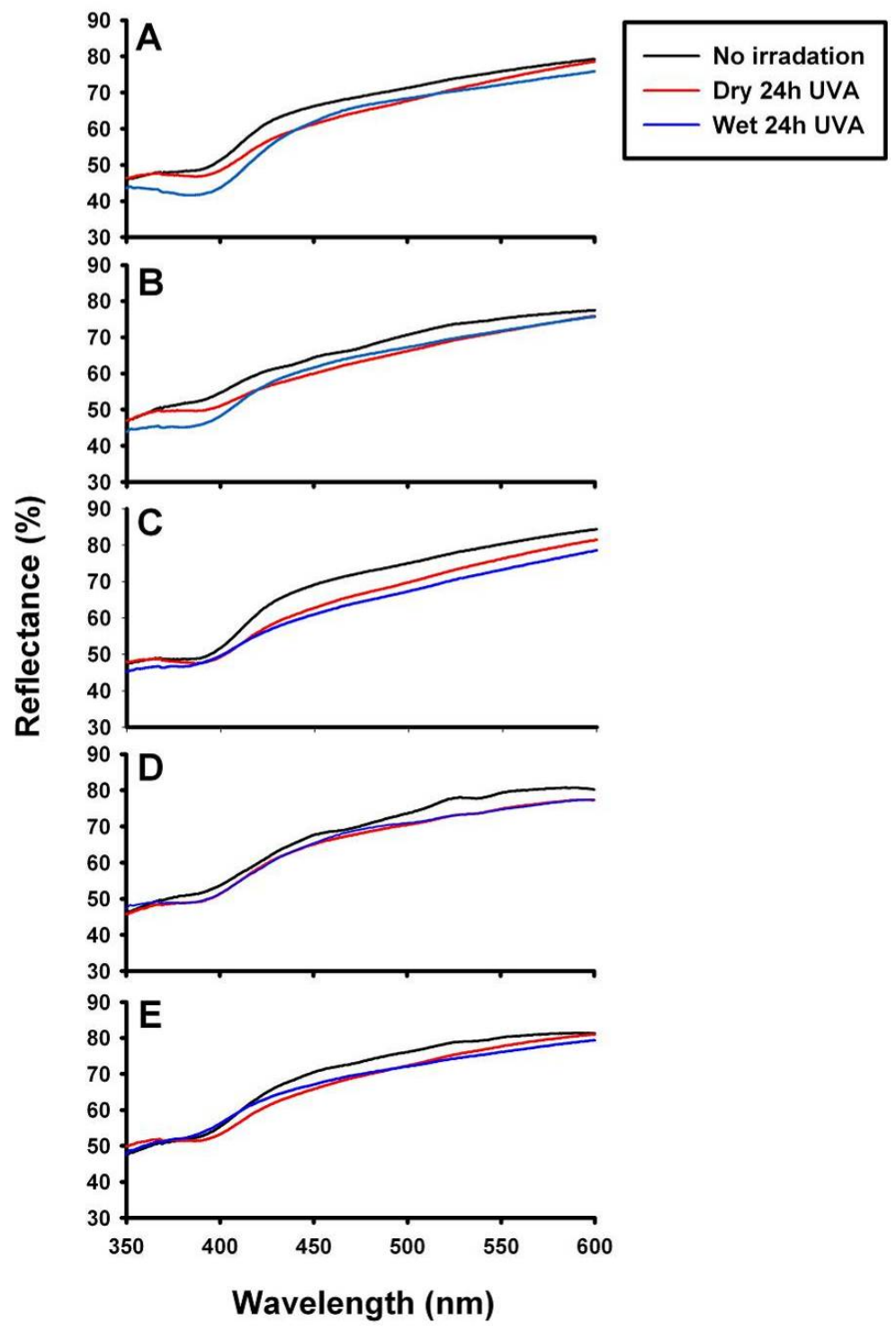

Figure 4.4 Reflectance spectra of solid fibres of five cultivars of $P$. tenax before (black curve) and after 24 hrs of UVA irradiation under dry (red curve) or wet conditions (blue curve): Arawa (A), Ngaro (B), Tapamangu (C), Mawaru (D) and Potaka (E). 


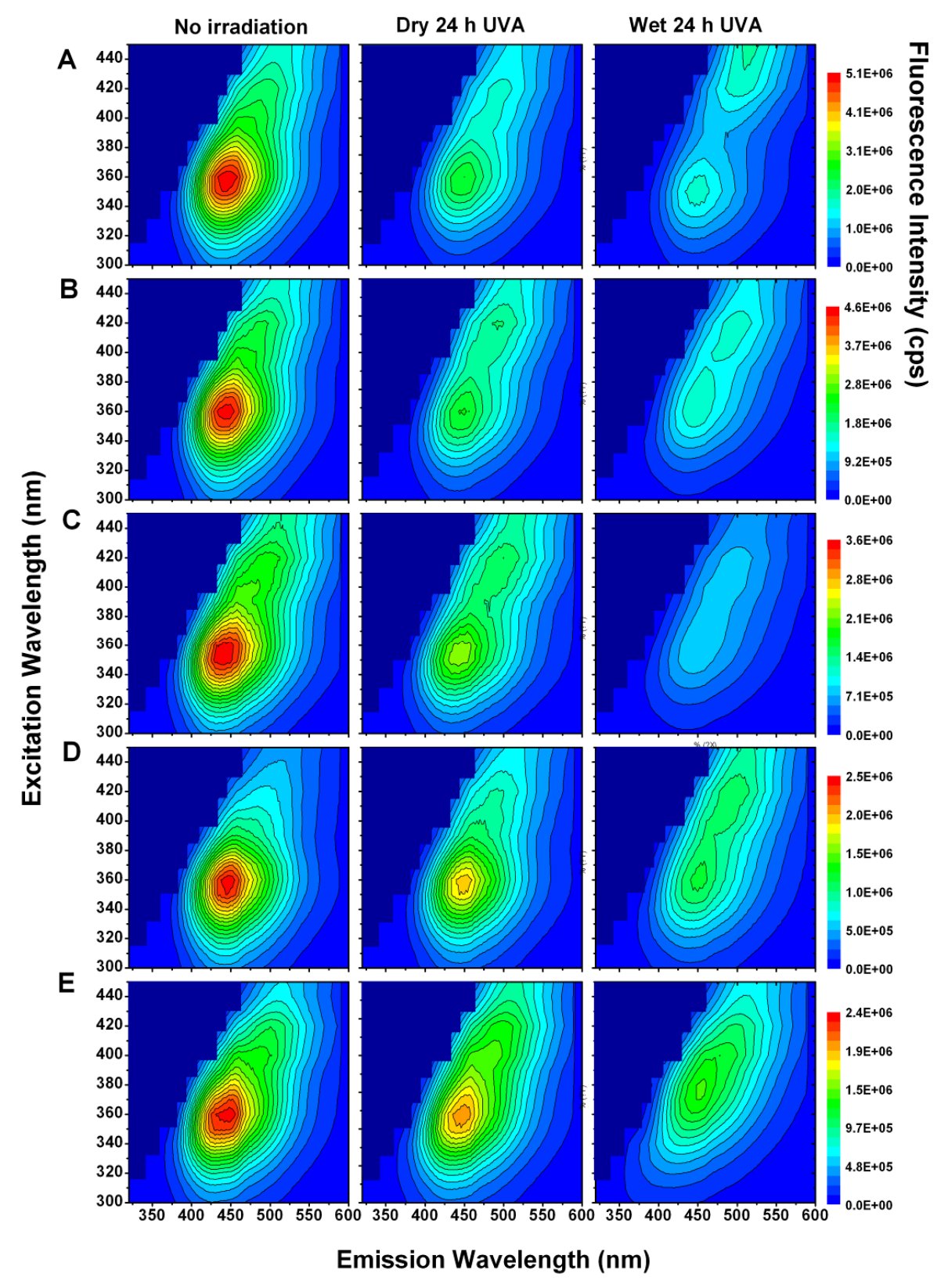

Figure 4.5 3D-fluorescence spectra of solid fibres of five cultivars of $P$. tenax before (left side) and after 24 hrs of UVA irradiation, dry (middle) or wet (right side): Arawa (A), Ngaro (B), Tapamangu (C), Mawaru (D) and Potaka (E). 
In summary, solid fibres of $P$. tenax emit fluorescence that is characteristic of 7hydroxycoumarins. Exposure of the fibres to UVA radiation caused photoyellowing of the fibre, manifested as reductions in reflectance in the blue region of the visible spectrum and loss of the coumarin fluorophores, accompanied by the formation of photoproducts that absorb and/or emit at longer wavelengths. Irradiation in the wet state was generally found to result in more photodegradation, contributing to an accelerated photoyellowing. This has also been observed in the light fading of cotton textiles with reactive dyes, in which the rapid wet degradation was shown to result from the oxidation of dyes containing electron donating substituents, whereas slower photodamage appear to involve reduction reactions ${ }^{[120]}$. Thus, the photochemical behaviours of Phormium fibres described here have suggested photooxidation as an important degradation mechanism. Significant intercultivar differences were also found.

\section{Photoreactions of 7-Hydroxycoumarins}

The changes in the fluorescence spectra of two standard 7-hydroxycoumarins in aqueous solution were also investigated, viz. 6,7-dihydroxycoumarin and 7hydroxy-6-methoxycoumarin, resulting from UVA irradiations over times from 0 to $96 \mathrm{hrs}$ at $24 \mathrm{hrs}$ intervals. The spectral changes in their absorbance and fluorescence are shown in Figure 4.6 and Figure 4.7, respectively. A summary of observations is as follows:

- For both hydroxycoumarins, the most notable feature observed upon the initial $24 \mathrm{hrs}$ of irradiation was a substantial reduction in the absorbance maxima, together with visually observable yellowing of the solutions. The relative rates of these changes in the two coumarins followed the trend: 7hydroxy-6-methoxycoumarin $>$ 6,7-dihydroxycoumarin. The most pronounced change was observed with 7-hydroxy-6-methoxycoumarin, with photoproducts absorbing and overlapping across the entire UV-Vis spectrum and the fluorescence was reduced to undetectable levels. It should be noted that the two 7-hydroxycoumarins have identical absorption spectra $\left(A_{\max }=350 \mathrm{~nm}\right)$ absorbing strongly in the spectral 
region covered by the output of the UVA source $(350-400 \mathrm{~nm})$. Their relative initial rates of degradation were based on the decreases in absorbance at $350 \mathrm{~nm}$.

- For 6,7-dihydroxycoumarin, a secondary absorption band or a shoulder was observed at shorter wavelengths from $280 \mathrm{~nm}$ to $290 \mathrm{~nm}$. Some coumarins subjected to UV radiation have been previously reported to undergo dimerisation and a number of these photodimers absorb maximally at around $280 \mathrm{~nm}^{[109]}$.

- The two coumarins have different yields of fluorescence: 7-hydroxy-6methoxycoumarin $>$ 6,7-dihydroxycoumarin (Figure 4.7). With the irradiated 6,7-dihydroxycoumarin solution, the observed spectral shift in the emission to shorter wavelength at $410 \mathrm{~nm}$, is consistent with the formation of photoproducts emitting at shorter wavelengths ${ }^{[109,121]}$.

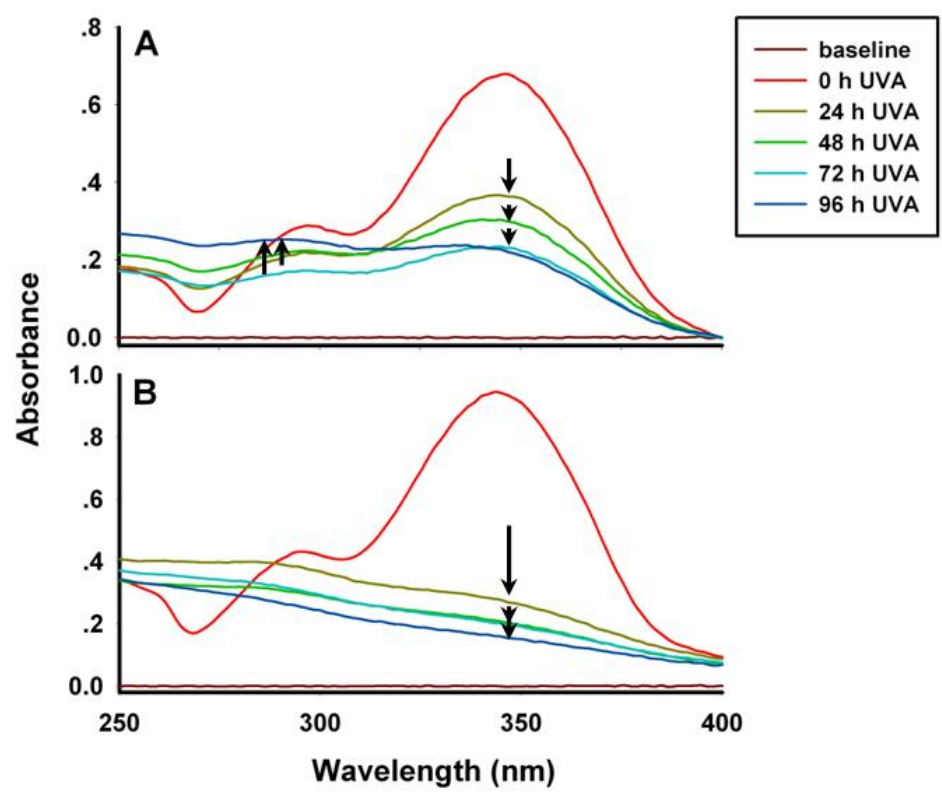

Figure 4.6 Absorption spectra of saturated aqueous solution of 6,7dihydroxycoumarin (A) and 7-hydroxy-6-methoxycoumarin (B) after UVA irradiation from 0 to $96 \mathrm{hrs}$ at $24 \mathrm{hrs}$ intervals 


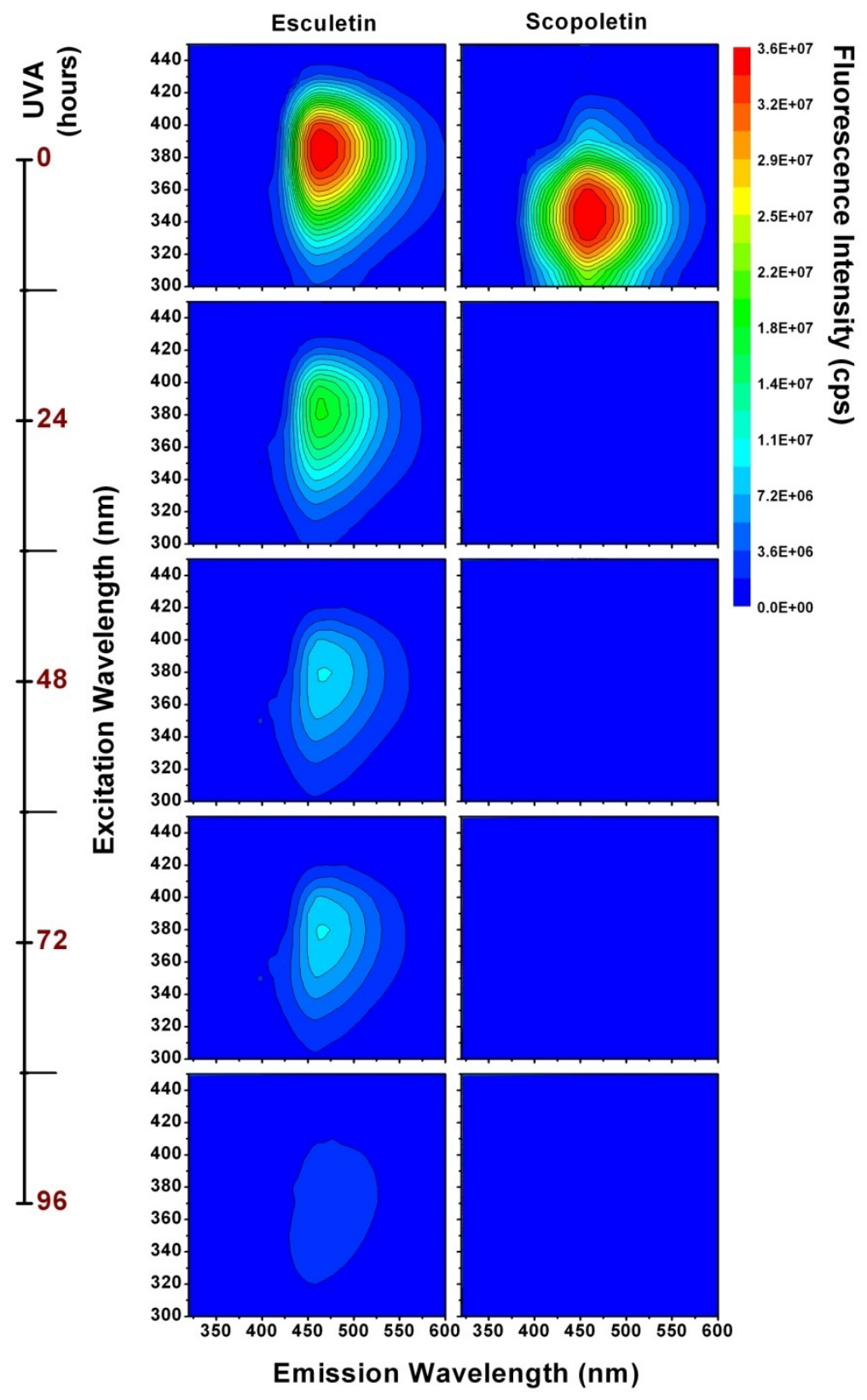

Figure 4.7 3D-fluorescence spectra of aqueous solutions of 6,7dihydroxycoumarin (esculetin, 20-fold diluted from original solution) and 7-hydroxy6-methoxycoumarin (scopoletin, 800-fold diluted), after UVA irradiation from 0 to $96 \mathrm{hrs}$ at $24 \mathrm{hrs}$ intervals 


\subsubsection{Superoxide and Peroxide Assays}

To further elucidate the contribution of reactive oxygen species (ROS) to the photooxidation of Phormium fibres, the kinetics of the production of hydrogen peroxide and superoxide radicals, were determined for fibres of the five cultivars and two standard 7-hydroxycoumarins in aqueous solution in this work.

\section{Phormium Fibres}

The fibres of all the five cultivars of $P$. tenax showed increasing concentrations of hydrogen peroxide in the water in contact with the fibres as a result of exposure to increasing doses of UVA radiation as evident in Figure 4.8.

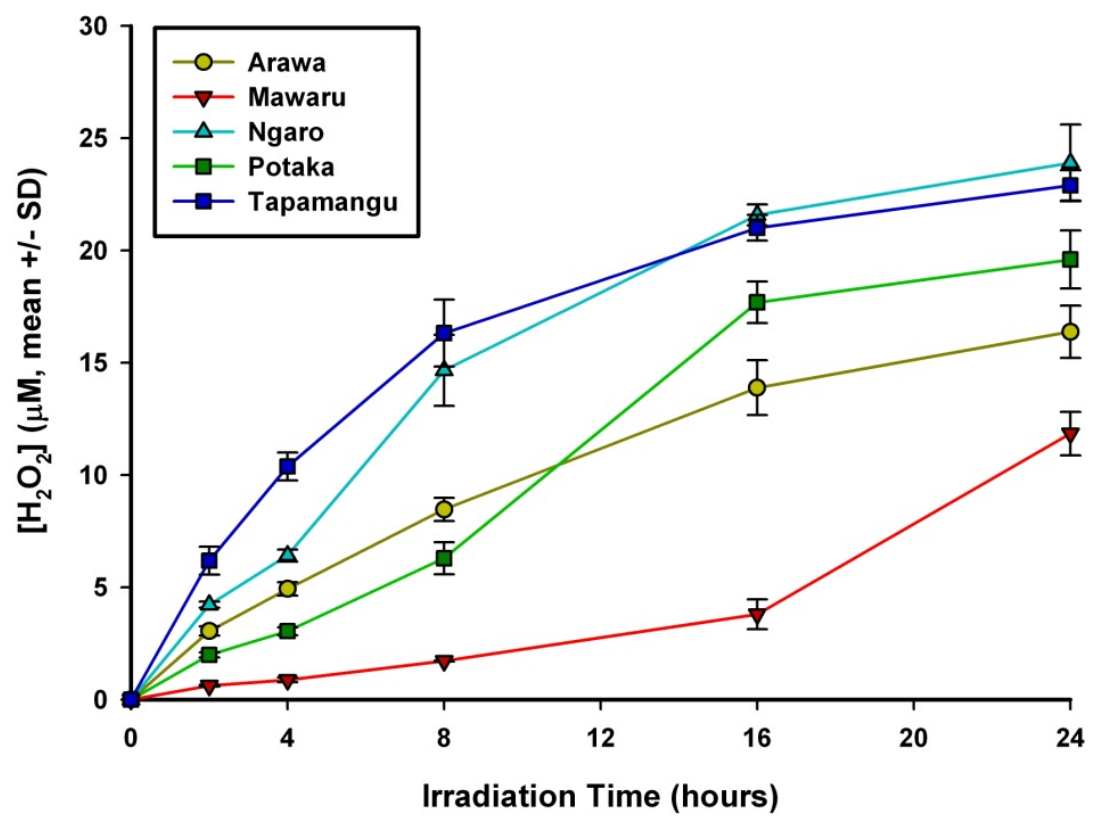

Figure 4.8 Production of $\mathrm{H}_{2} \mathrm{O}_{2}$ in wet UVA-irradiated Phormium fibres. $\mathrm{H}_{2} \mathrm{O}_{2}$ is shown as concentration $(\mu \mathrm{M})$ in the water from irradiated fibre over exposure time from 0 to $24 \mathrm{hrs}$. 
However, the yields of $\mathrm{H}_{2} \mathrm{O}_{2}$ produced in each cultivar differed during the irradiation periods. Generally, cultivars Ngaro and Tapamangu produced significantly more $\mathrm{H}_{2} \mathrm{O}_{2}$ than that from Mawaru, and the peroxide yields of Arawa and Potaka were intermediate. Since the "Fenton Reaction" provides a route of converting $\mathrm{H}_{2} \mathrm{O}_{2}$ to hydroxyl radicals (Reaction 5.1), this intercultivar difference in $\mathrm{H}_{2} \mathrm{O}_{2}$ production could be due, at least in part, to the different amounts of ferrous ions in fibres of different cultivars.

$$
\mathrm{Fe}^{2+}+\mathrm{H}_{2} \mathrm{O}_{2} \rightarrow \mathrm{Fe}^{3+}+\mathrm{OH}^{\bullet}+\mathrm{OH}^{-}
$$

A quantitative determination of iron contents of the five cultivars conducted in Chapter 6 supports this hypothesis.

Also, three of the five cultivars, i.e. Mawaru, Potaka and Tapamangu, were selected as representatives giving low to high yields of $\mathrm{H}_{2} \mathrm{O}_{2}$ associated with the detection of superoxide radicals. Figure 4.9 shows that adding superoxide dismutase (SOD) to the tested fibre samples immediately after the UVA irradiation resulted in significant higher levels of $\mathrm{H}_{2} \mathrm{O}_{2}$. Irradiation of SOD in the absence of fibre or with the addition of catalase produced no detectable $\mathrm{H}_{2} \mathrm{O}_{2}$. Since SOD and catalase are highly specific enzymes for superoxide and $\mathrm{H}_{2} \mathrm{O}_{2}$, respectively, it is evident that Phormium fibres generated $\mathrm{O}_{2}{ }^{--}$on exposure to UVA radiation. Their relative yields of $\mathrm{O}_{2}{ }^{--}$are determined by the incremental $\mathrm{H}_{2} \mathrm{O}_{2}$ yields and can be ranked following the order: Mawaru $>$ Potaka $\approx$ Tapamangu (Figure 4.9). 


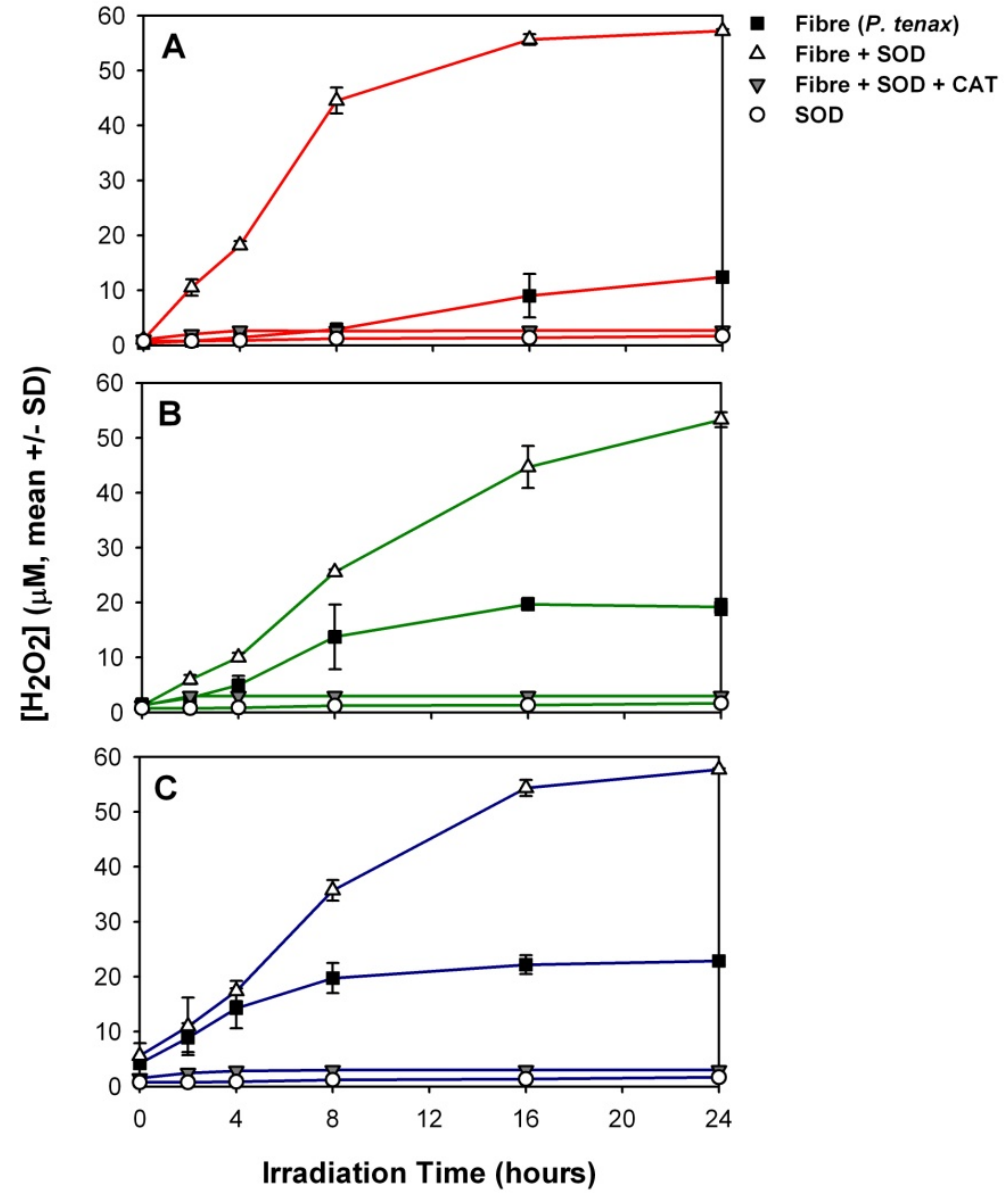

Figure 4.9 The effect of superoxide dismutase (SOD) and catalase (CAT) on the photoformation of $\mathrm{H}_{2} \mathrm{O}_{2}$ by wet UVA-irradiated Phormium fibres of three cultivars: Mawaru (A), Potaka (B) and Tapamangu (C). $\mathrm{H}_{2} \mathrm{O}_{2}$ is shown as concentration $(\mu \mathrm{M})$ in the water from irradiated fibre over exposure time from 0 to $24 \mathrm{hrs}$. 


\section{7-Hydroxycoumarins}

The peroxide and superoxide assays were also performed on the two hydroxylated coumarins studied in aqueous solutions during the initial $4 \mathrm{hrs}$ of UVA irradiation. The generation of $\mathrm{H}_{2} \mathrm{O}_{2}$, with or without the addition of SOD, for 6,7dihydroxycoumarin and 7-hydroxy-6-methoxycoumarin are shown in Figure 4.10. The results are described as below:

- Both coumarins showed generation of $\mathrm{H}_{2} \mathrm{O}_{2}$ on exposure to UVA radiation, with the maximum yields reached after $4 \mathrm{hrs}$ of irradiation. 6,7dihydroxycoumarin displayed a continuous increase in yielding $\mathrm{H}_{2} \mathrm{O}_{2}$ concentration while the yields for 7-hydroxy-6-methoxycoumarin reached a plateau after an initial rapid production of $\mathrm{H}_{2} \mathrm{O}_{2}$ in the first $2 \mathrm{hrs}$.

- It is apparent that 7-hydroxy-6-methoxycoumarin produced a significantly higher concentration of $\mathrm{H}_{2} \mathrm{O}_{2}$ than 6,7-dihydroxycoumarin on exposure to UVA radiation. This implies that 7-hydroxy-6-methoxycoumarin is a more active photosensitiser of oxidation than 6,7-dihydroxycoumarin in water, an observation that can be rationalised in terms of their relative redox potentials (see the following mechanism analysis). Their relative initial rates of $\mathrm{H}_{2} \mathrm{O}_{2}$ production during the first 1 to $2 \mathrm{hrs}$ of irradiation can also be correlated to the previous UV and fluorescence spectroscopic observations. 7-hydroxy-6-methoxycoumarin, which produced the highest yield of peroxide, also demonstrated a more rapid yellowing and photodegradation of fluorophores (see Figure 4.7).

- The addition of SOD to each irradiated sample immediately after irradiation induced only slightly higher concentration of $\mathrm{H}_{2} \mathrm{O}_{2}$, indicating a very rapid conversion of $\mathrm{O}_{2}{ }^{--}$into $\mathrm{H}_{2} \mathrm{O}_{2}$ in the aqueous environment in which the two 7-hydroxycoumarins were irradiated. No observable $\mathrm{H}_{2} \mathrm{O}_{2}$ was seen after adding catalase, showing there were no other oxidising species produced during irradiation. 

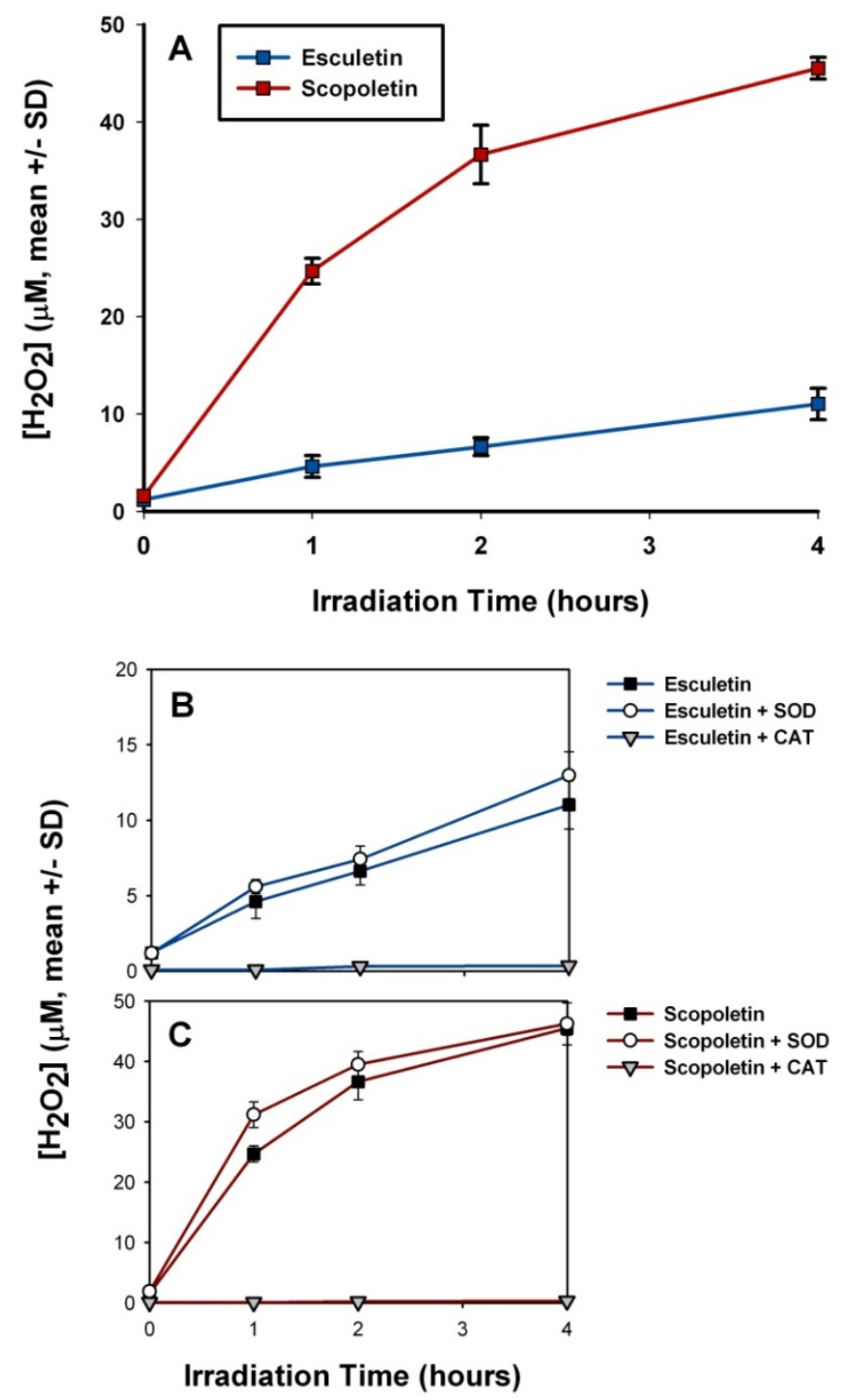

Figure 4.10 Photogeneration of $\mathrm{H}_{2} \mathrm{O}_{2}$ by saturated aqueous solutions of 7hydroxylated coumarins $(A)$ and the effect of superoxide dismutase (SOD) and catalase (CAT) on 6,7-dihydroxycoumarin (esculetin, B) and 7-hydroxy-6methoxycoumarin (scopoletin, C). $\mathrm{H}_{2} \mathrm{O}_{2}$ is shown as concentration $(\mu \mathrm{M})$ in the irradiated sample over exposure time from 0 to $4 \mathrm{hrs}$. 


\section{Mechanism of Photooxidation}

As discussed in the introduction to this chapter, there are two mechanisms by which ROS can be produced by the photoreaction of a photosensitiser in the excited state with molecular oxygen in the ground state. The formation of singlet oxygen results from the energy transfer from sensitisers in the excited triplet, and only occurs when the energy gap between the triplet state and ground state of the sensitiser is greater than that of singlet oxygen, $0.977 \mathrm{eV}^{[122]}$. Since the fluorescence quantum yields of coumarin fluorophores are high, it is inferred their triplet state yields are low, and give small yields of singlet oxygen. Also, the lifetime of singlet oxygen is of the order of $2 \mu \mathrm{s}^{[122]}$ in aqueous environments; so even if small quantities of singlet oxygen are generated, their involvement in subsequent reactions will be minor and thereby were not determined in this study.

The other type of photooxidation is initiated by the transfer of an electron between the excited singlet-state sensitisers to ground-state molecular oxygen, and is termed photoinduced electron transfer (PET). The energetics and dynamics of PET are governed by the energy of the photoexcited sensitising species $\left(\mathrm{E}^{*}\right)$, its ground state oxidation potential $\left(\mathrm{E}_{\mathrm{ox}}\right)$, the redox potential of oxygen $\left(\mathrm{E}_{\mathrm{O}_{2} / \mathrm{O}_{2}^{-}}=\right.$ $-0.82 \mathrm{~V}_{\mathrm{SCE}}$, where SCE is the standard calomel electrode), the nature and polarity of the medium and Coulombic effects, with the Gibbs energy $(\Delta G)$ for electron transfer given by:

$$
\Delta \mathrm{G}=\mathrm{E}_{\mathrm{ox}}-\mathrm{E}_{\mathrm{O}_{2} / \mathrm{O}_{2}^{-}}-\mathrm{E}^{*}+\mathrm{E}_{\mathrm{coul}}
$$

where $\mathrm{E}_{\text {coul }}$ is the energy of coulombic stabilisation between partners of the ion pair produced. In a polar environment, $\mathrm{E}_{\text {coul }}$ has a value $\sim 0.03 \mathrm{eV}^{[123,124]}$.

The $\mathrm{E}_{\text {ox }}$ of hydroxycoumarins are relatively low, e.g. $644 \mathrm{mV}_{\mathrm{SCE}}$ and $239 \mathrm{mV}_{\mathrm{SCE}}$ for 7-hydroxy-4-methylcoumarin and 6, 7-dihydroxycoumarin respectively while their excited singlet states energies are high, ranging from $\sim 2.8 \mathrm{eV}$ for $6,7-$ dihydroxycoumarin ${ }^{[125]}$ to $3.2 \mathrm{eV}$ for 7-hydroxy-4-methylcoumarin ${ }^{[126]}$. Therefore, the Gibbs energies of the charge transfer reactions for 7-hydroxycoumarins 
predicted by equation 4.1 are approximately $-1.7 \mathrm{eV}$. These are sufficiently negative that the reaction rates are expected to be diffusion controlled at ambient temperature ${ }^{[123,124]}$.

Furthermore, hydroxycoumarins can undergo photoionisation (electron transfer) and/or photodeprotonation (proton transfer) of their 7-hydroxyl groups as the initial step of UVA-induced photodegradation, and the initial rates are dependent on both the structure of the compound and the nature of the solvent in which they were irradiated. In the present study, the dihydroxycoumarin esculetin has shown to be more photostable than scopoletin in water. However, when irradiated in aprotic solvents, where the hydrogen bonding interaction is limited, esculetin degrades more rapidly than scopoletin due to the more acid or proton-donating capability of the dihydroxycoumarin, and their decay rates also associate with the basicities or proton-accepting ability of the solvent ${ }^{[127]}$.

Thus, the coumarin-sensitised photooxidation of Phormium fibres appears to occur via Type II or the mechanism of electron transfer rather than Type I involving singlet oxygen. The generic reaction scheme is shown below. It involves the initial formation of an excited singlet state of coumarin, ${ }^{1} \mathrm{C}^{*}$, which undergoes a rapid electron transfer reaction with oxygen dissolved in water to produce the transient superoxide radical anion and/or hydrogen atom transfer produce hydroperoxy free radicals ${ }^{[100,128]}$ that disproportionate to give hydrogen peroxide:

$$
\begin{gathered}
{ }^{1} \mathrm{C}+\mathrm{h} v \rightarrow{ }^{1} \mathrm{C}^{*} \\
{ }^{1} \mathrm{C}^{*}+\mathrm{O}_{2} \rightarrow{ }^{1} \mathrm{C}^{\cdot+}+\mathrm{O}_{2}{ }^{\cdot-} \\
{ }^{1} \mathrm{C}^{*}+\mathrm{O}_{2} \rightarrow{ }^{1} \mathrm{C}^{\bullet}+\mathrm{HOO}^{\cdot}
\end{gathered}
$$

and/or

$$
\mathrm{O}_{2}{ }^{\cdot-}+\mathrm{H}_{2} \mathrm{O} \rightarrow \mathrm{HOO}^{\bullet}+\mathrm{OH}^{-}
$$

$$
2 \mathrm{HOO}^{\cdot} \rightarrow \mathrm{H}_{2} \mathrm{O}_{2}+\mathrm{O}_{2}
$$

The above reactions could also lead to the production of phenolic free radicals. These phenolic radicals tautomerise to give centres of electron density on ring 
carbon atoms that are susceptible to attack by oxygen or undergo radical-radical combination reactions to form di- and tri-phenolic species ${ }^{[90]}$. The detection of hydroxylated and dimerisation photoproducts in the UVA-irradiated aqueous 7hydroxycoumarins described below, supports this mechanism.

\subsubsection{Mass Spectrometrical Photoproduct Analysis}

As a further approach to elucidating the mechanism of the photooxidation of coumarins, a four-step ESI-mass spectrometric analysis was employed to track the formation of photoproducts of 6,7-dihydroxycoumarin and 7-hydroxy-6methoxycoumarin in aqueous solution over the duration of irradiation. First, ESIMS was utilised to analyse each irradiated sample. Then, molecular ions of interest were selected for structural examination based on fragmentation patterns produced by ESI-MS/MS. Subsequently, the relative abundance of each photoproduct was semi-quantitatively evaluated by reference to the unmodified molecule; and a putative photolysis scheme was given.

\section{ESI-MS Analysis}

The ESI-MS spectra of solutions of 6,7-dihydroxycoumarin after 0 to $24 \mathrm{hrs}$ of UVA irradiation are shown in Figure 4.11. Along with the dominant molecular ion $\left(\mathrm{MH}^{+}\right)$at $\mathrm{m} / \mathrm{z} 179$, there are peaks of interest at $\mathrm{m} / \mathrm{z} 195,197,355$ and 379 present in the irradiated samples in varying relative abundances. The ions at m/z 195 and 197 could be assigned to a mono-oxidised product $(\mathrm{M}+\mathrm{O})$ and a hydroxylated product $\left(\mathrm{M}+\mathrm{H}_{2} \mathrm{O}\right)$, respectively. The ion with $\mathrm{m} / \mathrm{z} 379$ is equivalent to a sodiated dimer of 6,7-dihydroxycoumarin while that with $\mathrm{m} / \mathrm{z} 355$ is a protonated photoproduct. This photoproduct could be conceived as a dehydrogenated dimer of 6,7-dihydroxycoumarin, but the structure of this product could not be determined from its ESI-mass spectrum alone. After $48 \mathrm{hrs}$ of irradiation (Figure 4.12 ), the spectrum of 6,7-dihydroxycoumarin shows only a very small proportion of the sodiated $2 \mathrm{M}$ dimer was formed. 


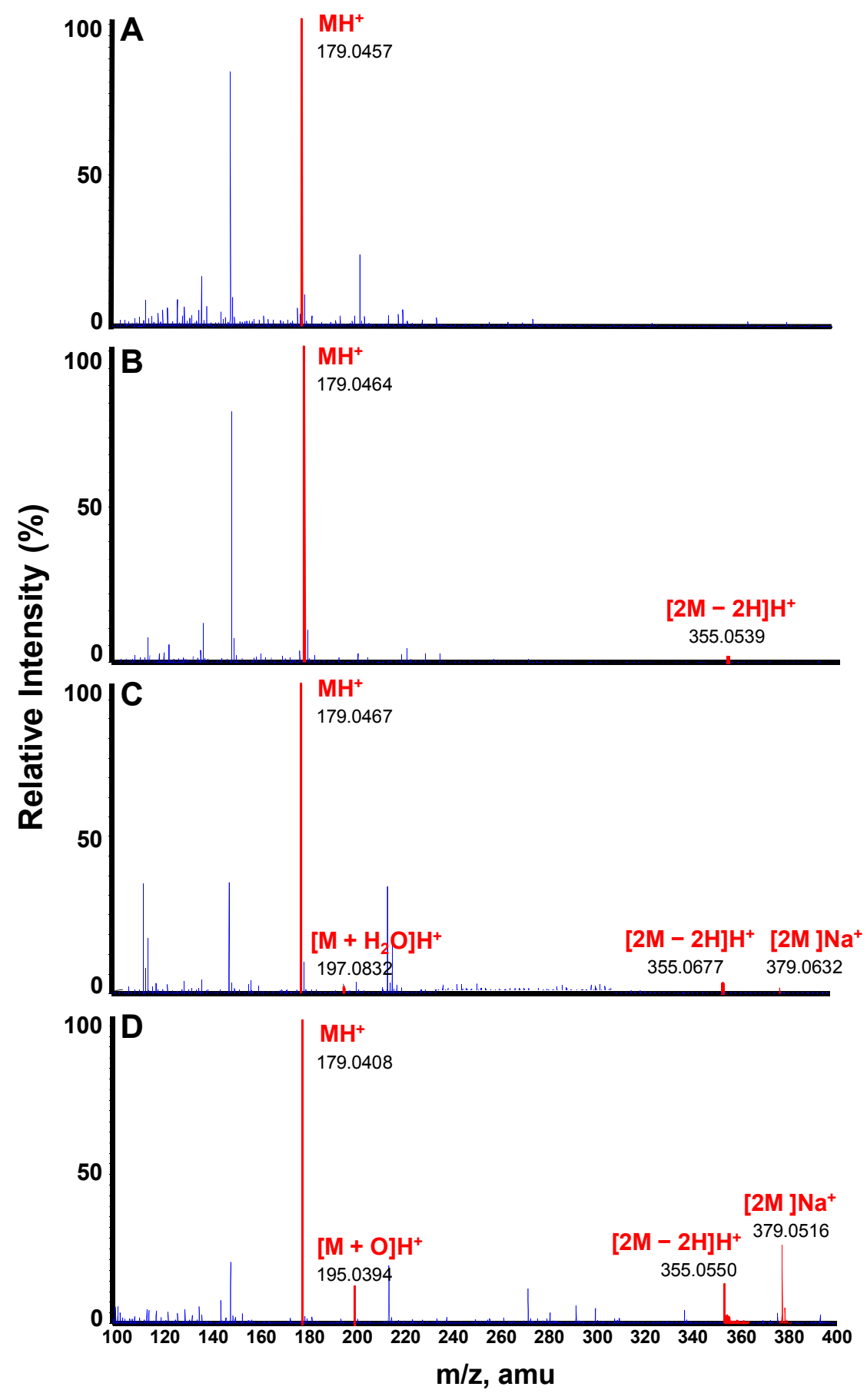

Figure 4.11 ESI-MS spectra of 6,7-dihydroxycoumarin after $0 \mathrm{hr}(\mathrm{A}), 8 \mathrm{hrs}(\mathrm{B}), 16$ hrs (C) and 24 hrs (D) of UVA irradiation, with molecular ions of the substrate $\left(\mathrm{MH}^{+}\right)$ and photoproducts labelled in red. 


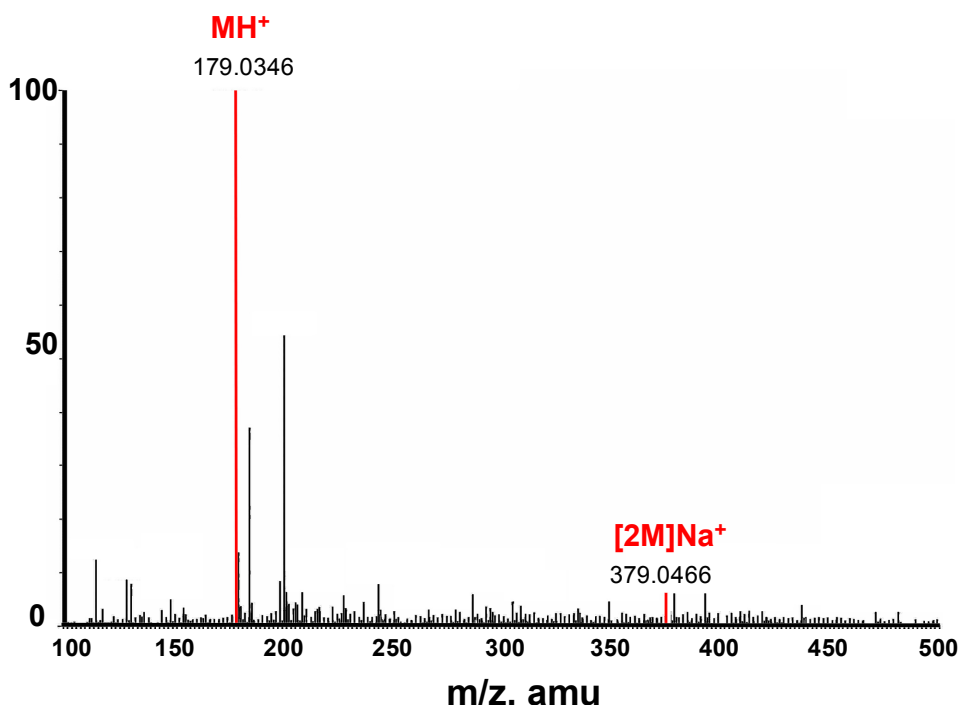

Figure 4.12 ESI-MS spectrum of 6,7-dihydroxycoumarin after $48 \mathrm{hrs}$ of UVA irradiation, with molecular ions of the substrate $\left(\mathrm{MH}^{+}\right)$and photoproducts labelled in red.

ESI-MS spectra of 7-hydroxy-6-methoxycoumarin after 24 and 48 hrs of UVA irradiation are shown in Figure 4.13. Along with the substrate ion $\left(\mathrm{MH}^{+}\right)$at $\mathrm{m} / \mathrm{z}$ 193, dimeric ions with $\mathrm{m} / \mathrm{z} 383,405$ and 407, together with a mono-oxidised $(\mathrm{M}+$ O) product ion at $\mathrm{m} / \mathrm{z} 209$ were observed in the spectra. The ion with $\mathrm{m} / \mathrm{z} 407$ is a dimeric sodiated ion $2 \mathrm{MNa}^{+}$, which can arise in two ways. Firstly, the electrospray process can form aggregates of the substrate molecule $(\mathrm{M})$ and these aggregates can form adducts with cations that are present in the solution, viz., $\mathrm{H}^{+}, \mathrm{Na}^{+}$and sometimes $\mathrm{K}^{+}$and $\mathrm{NH}_{4}^{+}$. These aggreates are most abundant in concentrated solutions as seen in Figure 4.13A. After irradiation, this ion at $\mathrm{m} / \mathrm{z} 407$ could also arise from the structural photodimers shown in Figure 4.18. The loss of two hydrogen atoms from the ion with $\mathrm{m} / \mathrm{z} 407$ gives rise to that with $\mathrm{m} / \mathrm{z} 405$, and the ion with $\mathrm{m} / \mathrm{z} 383$ is the protonated equivalent of the $\mathrm{m} / \mathrm{z} 405$ ion. The photodimer and ions formed from it were expected to increase in abundance as photolysis proceeded and this was observed in Figures $4.13 \mathrm{~B}$ and C. After $48 \mathrm{hrs}$ of irradiation, the sodiated ion with $\mathrm{m} / \mathrm{z} 405$ became the dominant ion present in the MS spectrum indicating that the substrate compound (M) of 7-hydroxy-6- 
methoxycoumarin was completely dimerised.

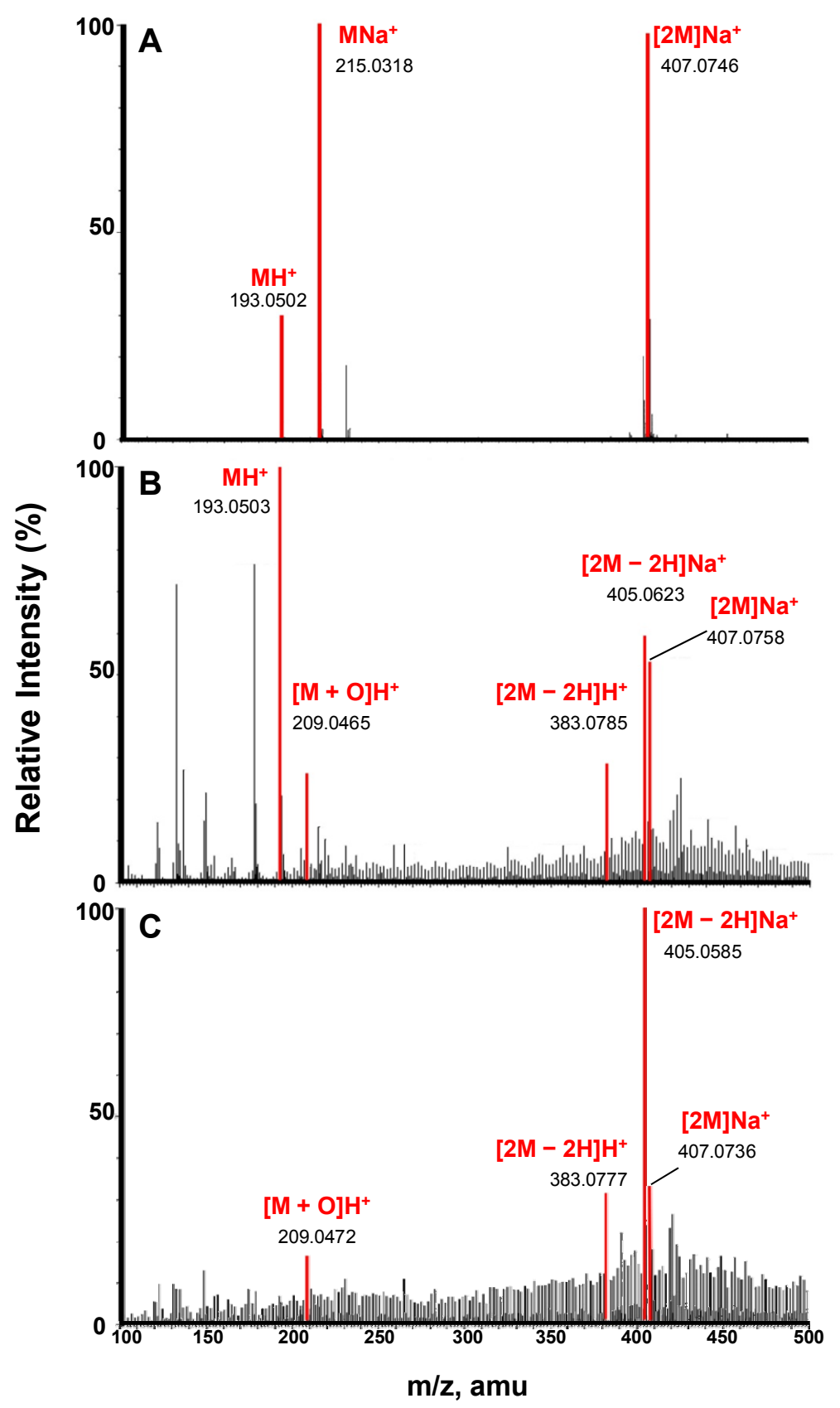

Figure 4.13 ESI-MS spectra of 7-hydroxy-6-methoxycoumarin after $0 \mathrm{hr}(\mathrm{A}) 24 \mathrm{hrs}$ (B) and $48 \mathrm{hrs}(\mathrm{C})$ of UVA irradiation, with molecular ions of substrate $\left(\mathrm{MH}^{+}\right)$and ions of photoproducts labelled in red. 


\section{MS/MS Structural Elucidation}

To confirm the identities of the photoproducts, molecular ions of interest were examined by ESI-MS/MS. It should be noted that although the formation of sodiated ions is favoured by the dimers, the $\mathrm{MNa}^{+}$were very stable and gave poor fragmentation, thus all structural assignments displayed here were based on fragmentation of the protonated species.

\section{Part I. 6,7-Dihydroxycoumarin (Esculetin)}

The MS/MS spectra of two oxidation products of 6,7-dihydroxycoumarin, $(\mathrm{M}+\mathrm{O})$ and $\left(\mathrm{M}+\mathrm{H}_{2} \mathrm{O}\right)$, are shown in Figure 4.14 with the molecular ions at $\mathrm{m} / \mathrm{z} 195$ (A) and 197 (B), respectively. For better comparisons between these two species, characteristic fragment ions occur in the mass spectrum of unirradiated 6,7dihydroxycoumarin (see Figure 3.10) are marked in red, while those observed only in the two photoproducts are in blue.

Figure 4.14A shows the MS/MS spectrum of mono-oxidised product $(\mathrm{M}+\mathrm{O})$, the fragmentation pattern of which is very similar to that of the unirradiated molecule except for two peaks of low abundance at m/z 177 and 151 which could result from the loss of $\mathrm{H}_{2} \mathrm{O}$ and $\mathrm{CO}_{2}$.

Figure 4.14B shows the MS/MS spectrum of hydroxylated product $\left(\mathrm{M}+\mathrm{H}_{2} \mathrm{O}\right)$. In addition to the fragment ions that were also observed on the unirradiated and mono-oxidised 6,7-dihydroxycoumarin, marked in red or blue, ions at m/z 133, 105 and 99 were also present. These daughter ions correspond to the loss of $\mathrm{CH}_{4} \mathrm{O}_{3}\left(\mathrm{CO}+2 \mathrm{H}_{2} \mathrm{O}\right), \mathrm{C}_{2} \mathrm{H}_{4} \mathrm{O}_{4}\left(2 \mathrm{CO}+2 \mathrm{H}_{2} \mathrm{O}\right)$ and $\mathrm{CH}_{6} \mathrm{O}_{5}\left(\mathrm{CO}_{2}+3 \mathrm{H}_{2} \mathrm{O}\right)$, and indicated additional functionality of hydroxyl groups. However, the position of hydroxylation indicated in the figure is representative only as it can not be determined by ESI-MS/MS. 

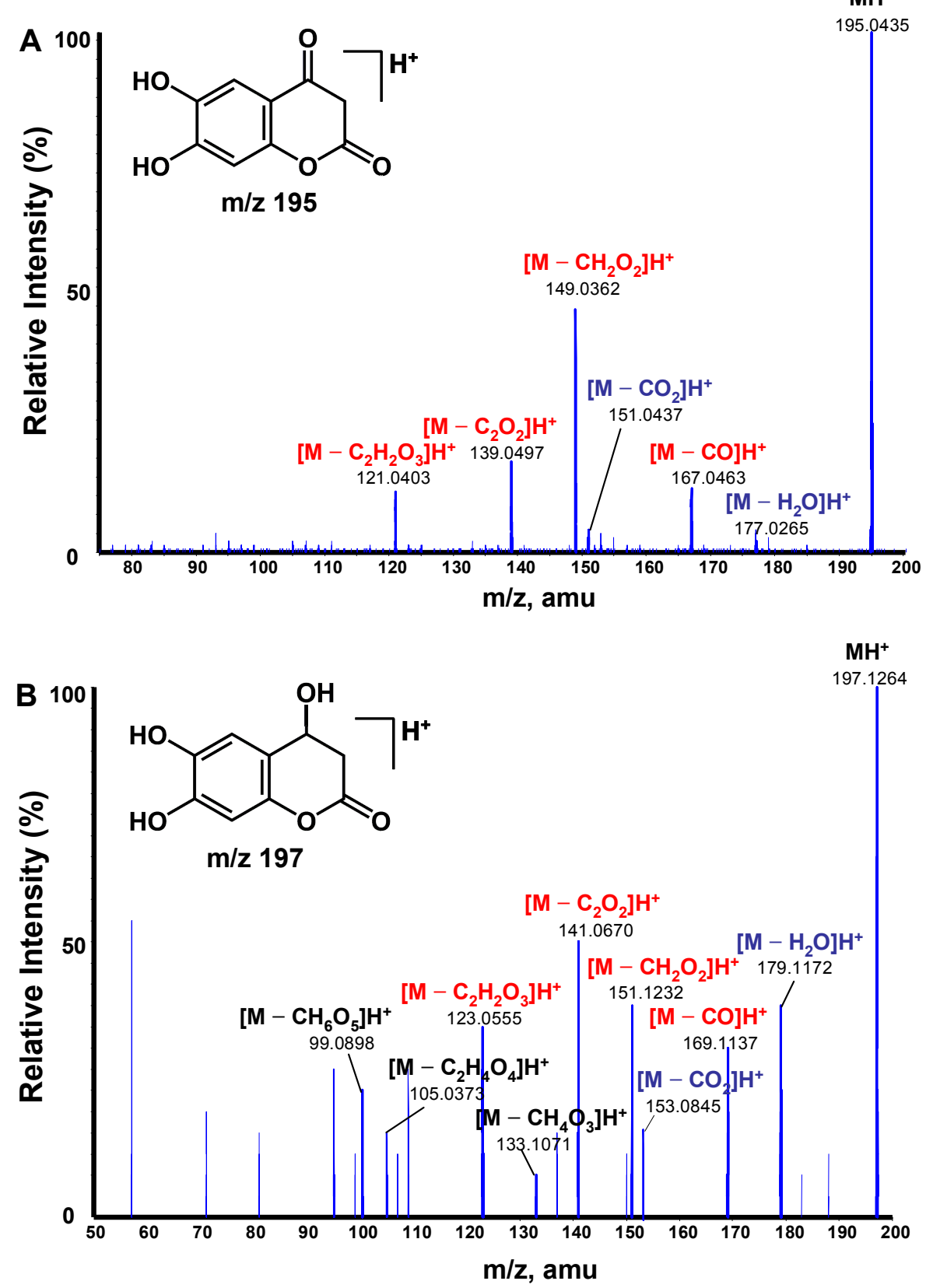

Figure 4.14 ESI-MS/MS spectra of the mono-oxidised $(M+O)$ product ion $(A)$ and the hydroxylated $\left(\mathrm{M}+\mathrm{H}_{2} \mathrm{O}\right)$ product ion $(\mathrm{B})$ of 6,7-dihydroxycoumarin, with characteristic fragments labelled (in red if the neutral loss was also observed in unirradiated sample; or in blue for those observed only in A and B). The structures drawn are speculative. 


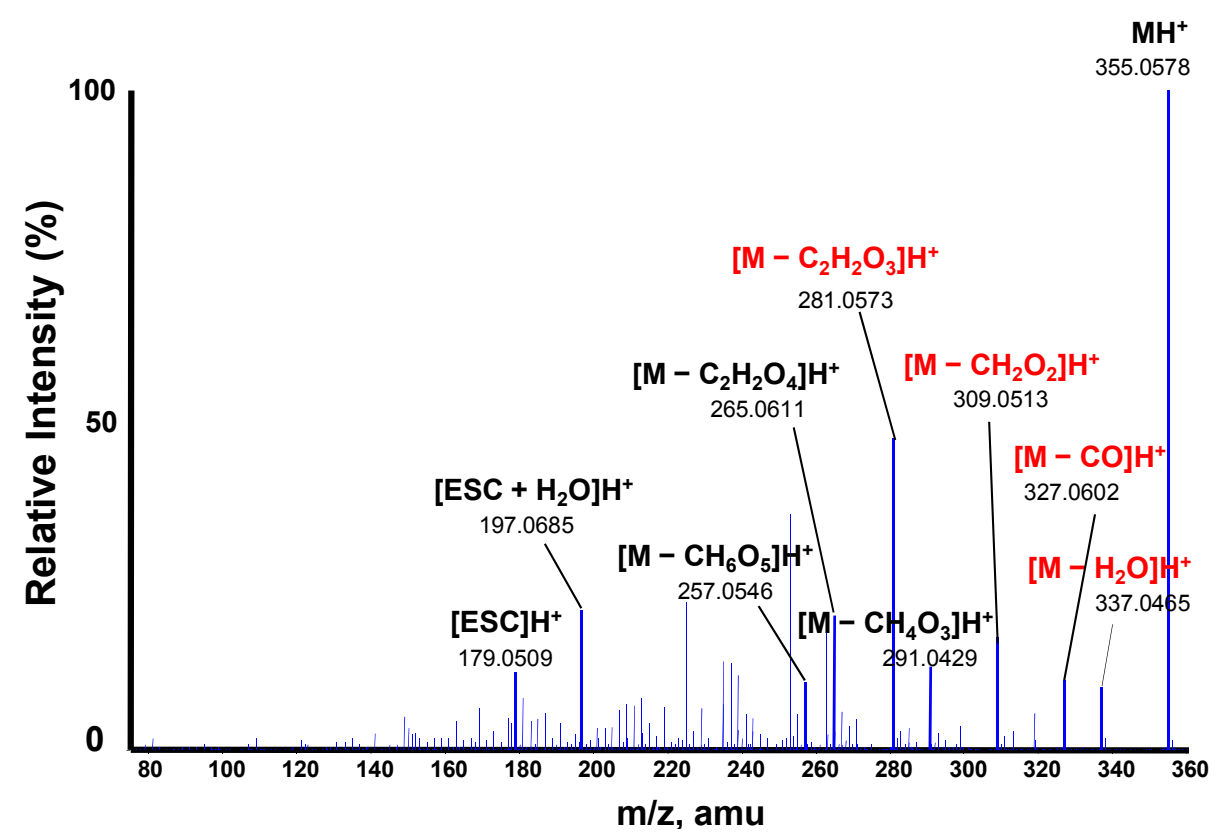

Figure 4.15 ESI-MS/MS spectrum of the $\mathrm{m} / \mathrm{z} 355$ product ion from 6,7dihydroxycoumarin, with characteristic fragment ions labelled (in red if the neutral loss was also observed in the unirradiated sample).

Figure 4.15 shows the MS/MS spectrum of the ion with $\mathrm{m} / \mathrm{z} 355$ from $6,7-$ dihydroxycoumarin. The structure of the ion could not be determined from the ESI-MS but is equivalent to a protonated dimer of 6,7-dihydroxycoumarin which has lost two atoms of hydrogen. A dimeric structure is based on previous reports on photocyclodimers derived from UV-irradiated coumarin ${ }^{[11]}$ and 5,7dimethoxycoumarin ${ }^{[109,110,129]}$, with details given in the following discussion of photodegradation pathways. Again, there were fragment ions characteristic of the unirradiated substrate (marked in red) at $\mathrm{m} / \mathrm{z}$ 337, 327, 309 and 281, which are produced from the loss of $\mathrm{H}_{2} \mathrm{O}, \mathrm{CO}, \mathrm{CH}_{2} \mathrm{O}_{2}\left(\mathrm{CO}+\mathrm{H}_{2} \mathrm{O}\right)$ and $\mathrm{C}_{2} \mathrm{H}_{2} \mathrm{O}_{3}(2 \mathrm{CO}+$ $\left.\mathrm{H}_{2} \mathrm{O}\right)$. Also apparent were fragment ions corresponding to the loss of $\mathrm{CH}_{4} \mathrm{O}_{3}(\mathrm{CO}$ $\left.+2 \mathrm{H}_{2} \mathrm{O}\right), \mathrm{C}_{2} \mathrm{H}_{2} \mathrm{O}_{4}\left(\mathrm{CO}+\mathrm{CO}_{2}+\mathrm{H}_{2} \mathrm{O}\right)$ and $\mathrm{CH}_{6} \mathrm{O}_{5}\left(\mathrm{CO}_{2}+3 \mathrm{H}_{2} \mathrm{O}\right)$, at m/z 291, 265, 257, respectively, which could be attributed to multiple hydroxylations on the parent coumarin. Fragments of the monomer (ESC) and a hydroxylated derivative $\left(\mathrm{ESC}+\mathrm{H}_{2} \mathrm{O}\right)$ at $\mathrm{m} / \mathrm{z} 179$ and 197 respectively, were also present. 


\section{Part II. 7-Hydroxy-6-Methoxycoumarin (Scopoletin)}

In the case of 7-hydroxy-6-methoxycoumarin, the mono-oxidised $(\mathrm{M}+\mathrm{O})$ product ion and the dimerisation product ion were observed in all two irradiated samples in varying relative abundances.

As shown in Figure 4.16, the fragmentation pattern of the $(\mathrm{M}+\mathrm{O})$ product ion is almost the same as that of 7-hydroxy-6-methoxycoumarin (see Figure 3.11), but with an additional oxygen in each fragment. The only fragment ion that was not observed in the unirradiated sample is at $\mathrm{m} / \mathrm{z} 135$, corresponding to the loss of $\mathrm{C}_{2} \mathrm{H}_{2} \mathrm{O}_{3}\left(2 \mathrm{CO}+\mathrm{H}_{2} \mathrm{O}\right)$.

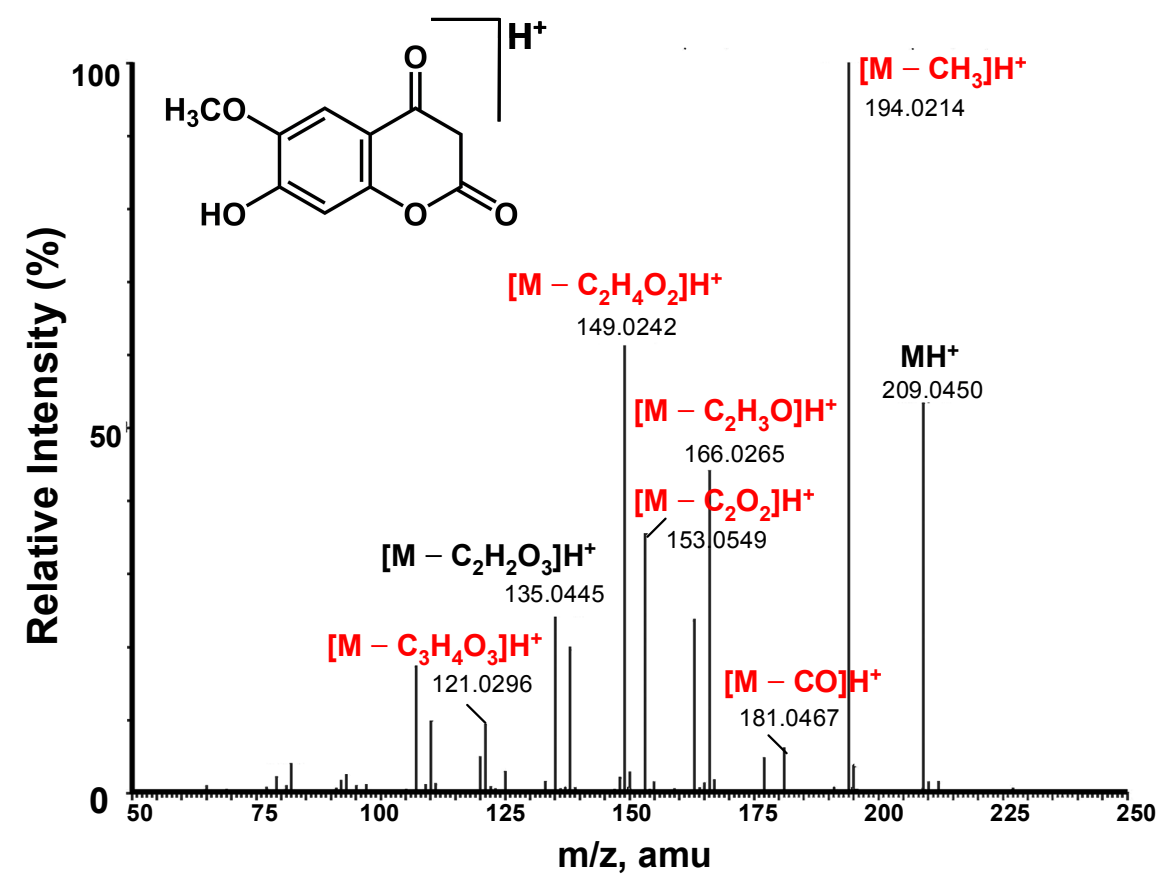

Figure 4.16 ESI-MS/MS spectrum of the mono-oxidised $(M+O)$ product ion of 7hydroxy-6-methoxycoumarin, with characteristic fragment ions labelled (in red if the neutral loss was also observed in the unirradiated sample). The structure of the product is indicative only. 


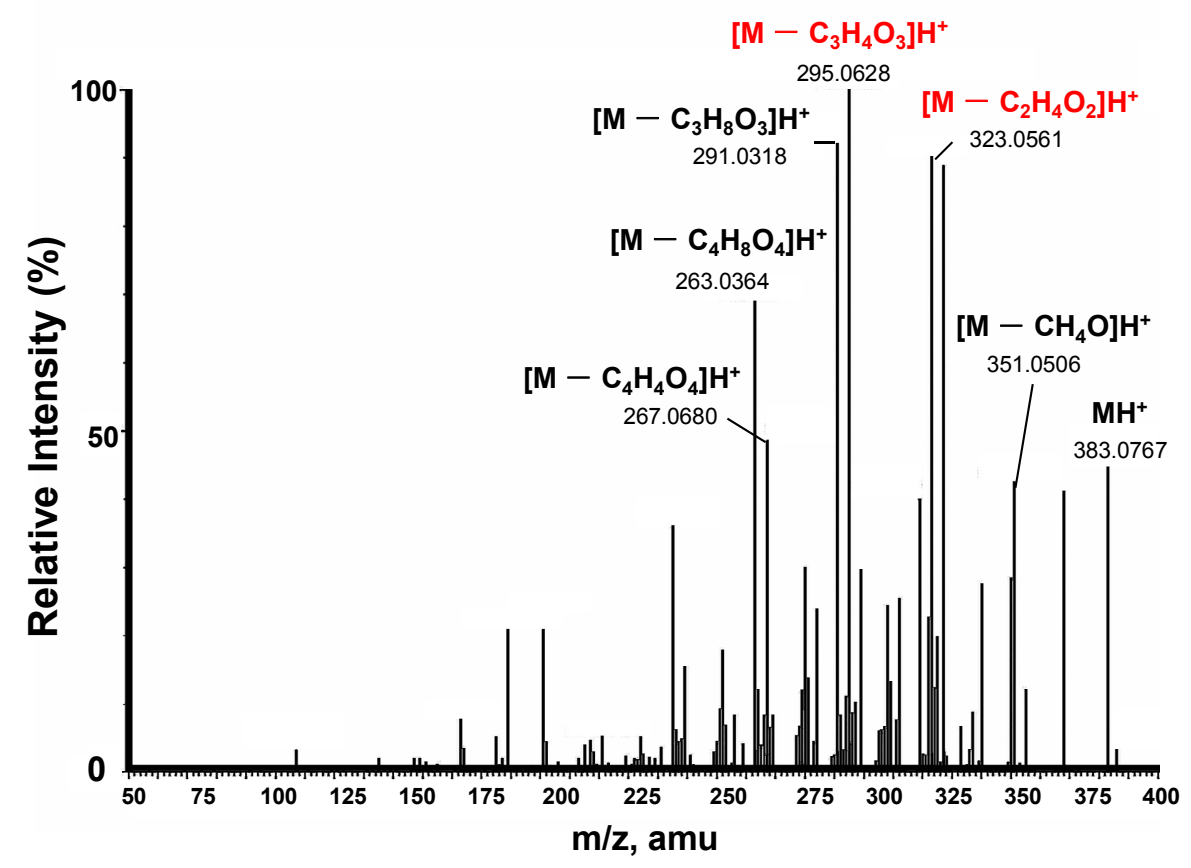

Figure 4.17 ESI-MS/MS spectrum of the $\mathrm{m} / \mathrm{z} 383$ product ion of 7-hydroxy-6methoxycoumarin, with characteristic fragment ions labelled (in red if the neutral loss was also observed in unirradiated sample).

Figure 4.17 shows the MS/MS spectrum of the product from the dimerisation of 7-hydroxy-6-methoxycoumarin. As for the dimer of 6,7-dihydroxycoumarin described earlier, the structure of the photodimer could not be determined with certainty by ESI-MS/MS. The ion with $\mathrm{m} / \mathrm{z} 383$ is equivalent to a protonated dimer of 7-hydroxy-6-methoxycoumarin which has lost two atoms of hydrogen. Besides the two fragment ions at $\mathrm{m} / \mathrm{z} 323$ and 295 that are characteristic of 6,7dihydroxycoumarin, daughter ions occurred at m/z 291, 267 and 263 which correspond to the loss of $\mathrm{C}_{3} \mathrm{H}_{8} \mathrm{O}_{3}\left(2 \mathrm{CH}_{3} \mathrm{OH}+\mathrm{CO}\right), \mathrm{C}_{4} \mathrm{H}_{4} \mathrm{O}_{4}\left(\mathrm{CH}_{3} \mathrm{OH}+3 \mathrm{CO}\right)$, $\mathrm{C}_{4} \mathrm{H}_{8} \mathrm{O}_{4}\left(2 \mathrm{CH}_{3} \mathrm{OH}+2 \mathrm{CO}\right)$.

The ESI-MS/MS spectra provide strong evidence for the UVA-induced formation of oxidised and dimerised products of 7-hydroxycoumarins in aqueous solution. The diagnostic fragment ions used in this study are summarised in Table 4.1. 
Table 4.1 Diagnostic ions (m/z) used in ESI-MS/MS identification of photoproducts of UVA-irradiated aqueous 6,7-dihydroxycoumarin (esculetin) and 7-hydroxy-6-methoxycoumarin (scopoletin). Daughter ions result from the loss of neutral molecules from $\mathrm{MH}^{+}$.

\begin{tabular}{|c|c|c|c|c|c|c|c|c|c|c|c|c|}
\hline \multirow[b]{2}{*}{ Esculetin (ESC) } & \multirow[b]{2}{*}{$\mathrm{MH}^{+}$} & \multicolumn{11}{|c|}{ Neutral Loss Units } \\
\hline & & $\mathrm{H}_{2} \mathrm{O}$ & co & $\mathrm{CO}_{2}$ & $\mathrm{CH}_{2} \mathrm{O}_{2}$ & $\mathrm{C}_{2} \mathrm{O}_{2}$ & $\mathrm{CH}_{4} \mathrm{O}_{3}$ & $\mathrm{C}_{2} \mathrm{H}_{2} \mathrm{O}_{3}$ & $\mathrm{C}_{2} \mathrm{H}_{2} \mathrm{O}_{4}$ & $\mathrm{C}_{2} \mathrm{H}_{4} \mathrm{O}_{4}$ & $\mathrm{CH}_{6} \mathrm{O}_{5}$ & \\
\hline ESC & 179 & 161 & 151 & & 133 & 123 & & 105 & & & & \\
\hline$E S C+O$ & 195 & 177 & 167 & 151 & 149 & 139 & & 121 & & & & \\
\hline $\mathrm{ESC}+\mathrm{H}_{2} \mathrm{O}$ & 197 & 179 & 169 & 153 & 151 & 141 & 133 & 123 & & 105 & 99 & \\
\hline $2 \mathrm{ESC}-2 \mathrm{H}$ & 355 & 337 & 327 & & 309 & & 291 & 281 & 265 & & 257 & \\
\hline Scopoletin (SCP) & $\mathrm{MH}^{+}$ & $\mathrm{CH}_{3}$ & co & $\mathrm{CH}_{4} \mathrm{O}$ & $\mathrm{C}_{2} \mathrm{H}_{3} \mathrm{O}$ & $\mathrm{C}_{2} \mathrm{O}_{2}$ & $\mathrm{C}_{2} \mathrm{H}_{4} \mathrm{O}_{2}$ & $\mathrm{C}_{2} \mathrm{H}_{2} \mathrm{O}_{3}$ & $\mathrm{C}_{3} \mathrm{H}_{4} \mathrm{O}_{3}$ & $\mathrm{C}_{3} \mathrm{H}_{8} \mathrm{O}_{3}$ & $\mathrm{C}_{4} \mathrm{H}_{4} \mathrm{O}_{4}$ & $\mathrm{C}_{4} \mathrm{H}_{8} \mathrm{O}_{4}$ \\
\hline SCP & 193 & 178 & 165 & & 150 & 137 & 133 & & 105 & & & \\
\hline $\mathrm{SCP}+\mathrm{O}$ & 209 & 194 & 181 & & 166 & 153 & 149 & 135 & 121 & & & \\
\hline $2 \mathrm{SCP}-2 \mathrm{H}$ & 383 & & & 351 & & & 323 & & 295 & 291 & 267 & 263 \\
\hline
\end{tabular}




\section{Relative Abundance}

For both 6,7-dihydroxycoumarin and 7-hydroxy-6-methoxycoumarin, it is clear that dimerisation was the dominant result from the photodegradation of aqueous 7-hydroxycoumarins. The dimer was observed to increase in abundance with increasing irradiation times, and pronounced changes were observed in 7hydroxy-6-methoxycoumarin, all of which formed the dimer after $48 \mathrm{hrs}$. 6,7Dihydroxycoumarin is comparatively more resistant to dimerisation. The $(\mathrm{M}+\mathrm{O})$ products were the second abundant photoproducts in the two irradiated 7hydroxycoumarins. For 6,7-dihydroxycoumarin, a hydroxylated product $(\mathrm{M}+$ $\mathrm{H}_{2} \mathrm{O}$ ) was also characterised as a trace component but only detected in the $16 \mathrm{hrs}$ irradiated sample, suggesting it may had occurred as an intermediate.

\section{Putative Photodegradation Pathways}

From the above analysis, two routes of UVA-induced photodegradation of 7hydroxycoumarins can be proposed as shown in Figure 4.18. However, the mechanism for formation of these photoproducts is not clear.

The $(\mathrm{M}+\mathrm{O})$ products may result from the attack of ROS such as hydrogen peroxide, as previously detected in the aqueous solutions of photolysed 7hydroxycoumarins, on the coumarin. In the case of 6,7-dihydroxycoumarin, the oxidation occurs via the formation of an unstable hydroxylated intermediate, which is consistent with previous observations of hydroxylation as the key photodegradation pathway leading to fluorescence changes in the photoyellowing of stilbenes ${ }^{[130]}$.

For the $2 \mathrm{M}$ dimerisation, in principle, as many as four isomers may be formed via the $[2 \pi \mathrm{s}+2 \pi \mathrm{s}]$ cycloaddition formation of a cyclobutane ring ${ }^{[111]}$ : syn head-tohead (syn-HH), syn head-to-tail (syn-HT), anti head-to-head (anti-HH) and anti head-to-tail (anti-HT). The syn- and anti-dimers are produced via singlet and triplet reactions respectively ${ }^{[129]}$. Previous studies of irradiated coumarins in various solvents have shown that the dominant dimers formed in polar solvents 
are syn- and anti-HH, while the formation of anti-HH is favoured in the presence of triplet sensitisers such as benzophenone ${ }^{[109-111,129]}$. For the minor dimers, synHT derives from nonsensitised photolysis, and anti-HT is obtained at low temperature $^{[34]}$. In light of the polar environment in which the two 7hydroxycoumarins were irradiated and the high yielding of hydrogen peroxide derived from excited singlet state reactions (see Section 4.3.2), the configuration of $s y n$ head-to-head was assumed to be the principal isomer of $2 \mathrm{M}$ product.

A pictorial representation of the formation of these products after photolysis is given in Figure 4.18. However, the structure of the $(2 \mathrm{M}-2 \mathrm{H})$ product formed in the ESI-mass spectrometre could not be determined. 


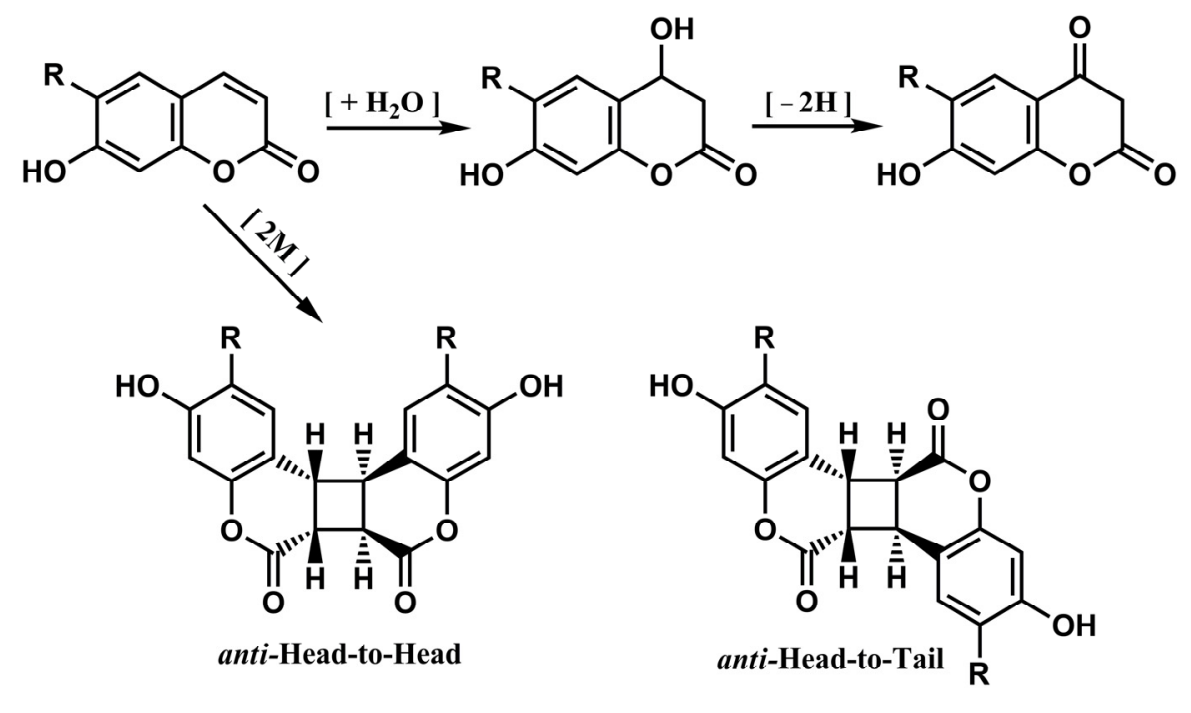<smiles>[R]c1cc2c(cc1O)OC(=O)[C@H]1[C@H]2[C@H]2C(=O)Oc3cc(O)c([R])cc3[C@H]21</smiles>

$s y n$-Head-to-Head

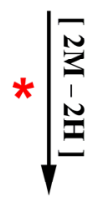$$
\text { ? }
$$

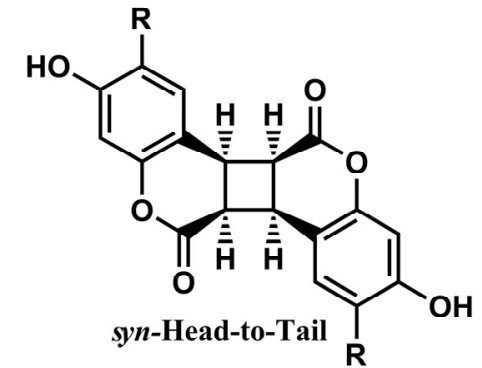

Figure 4.18 Putative photodegradation pathway of 7-dihydroxycoumarins $(\mathrm{R}=\mathrm{OH}$ or $\mathrm{OCH}_{3}$ ) in aqueous solution upon UVA irradiation, leading to the formation of mono-oxidation products and photodimers. ("product formed during ESI-mass spectrometry). 


\subsection{Summary of the Chapter}

In order to elucidate the role of naturally-occurring 7-hydroxycoumarins in the photoyellowing of Phormium fibres, fibres of five named cultivars were exposed to UVA radiation with a maximum output at $366 \mathrm{~nm}$ from 'blacklight' lamps and characterised by 3D-fluorescence and UV-Vis reflectance spectroscopy.

The irradiation of fibres resulted in a substantial loss of the blue fluorescence originating from 7-hydroxycoumarins, together with the occurrence of new fluorophores absorbing and emitting at longer wavelengths, both leading to the duller and yellow appearance of the fibre. A more rapid yellowing was observed when irradiating fibres in the wet state, which is consistent with previous reports on FWA-treated wool ${ }^{[96,97]}$. Additionally, the yellowing rates of Phormium fibres varied considerably in different cultivars. And there is a correlation between their relative photosensitivities and the resistance to fungal infection (see Chapter 2) for the five cultivars studied in this work.

Two standard 7-hydroxycoumarins were also investigated as important model compounds in aqueous solution and subjected to UVA-irradiation. Their absorption and fluorescence spectra showed a gradual fading of the fluorescence with increased exposure time. For 6,7-dihydroxycoumarin, a shift in the emission towards shorter wavelengths was also observed in the 3D-fluorescence spectra of irradiated samples. This change of fluorescence is attributed to the photoformation of higher molecular weight chromophores which was later confirmed by the photoproduct analysis of UVA-irradiated 6,7-dihydroxycoumarin and 7-hydroxy6-methoxycoumarin by ESI-MS.

Two primary photoproducts have been identified in aqueous solutions of the two 7-hydroxycoumarins during irradiation: a photodimer containing a linking cyclobutane ring and a monomeric photooxidation product. The oxidation products have not been reported previously and the characterisation in this study represents new knowledge. 
An assessment of the UVA-induced production of reactive oxygen species (ROS), i.e. hydrogen peroxide and superoxide radicals, as a function of irradiation time was also performed on Phormium fibres and the aqueous 7-hydroxycoumarins. The results suggest that the formation of at least some of the photoproducts is likely to result from the sensitised photooxidation via Type II or the mechanism of electron transfer interaction with coumarins in the excited singlet state. 


\section{Chapter 5}

\section{Chinese Handmade Paper: Fluorescence and Photodegradation}

In this chapter, another type of lignocellulosic material of cultural heritage value, Chinese handmade paper, is investigated and its fluorescence property and photochemical behaviour upon exposure to UVA radiation determined. The relative photostabilities of papers from several sources are assessed.

\subsection{Introduction to Paper Deterioration}

Paper is a matted/felted sheet of lignocellulosic fibres from pulped plant materials ${ }^{[33]}$. Paper deterioration is manifested as changes in optical and mechanical properties such as fibre strength, crystallinity or viscoelastic behaviour $^{[131]}$, associated with the release of volatile compounds such as acetic acid and furfural ${ }^{[132,133]}$. It is mainly due to the degradation of cellulose, hemicellulose and lignin components caused by several mechanisms ${ }^{[133,134]}$, and in many cases the primary reactions involved are a combination of acid hydrolysis and oxidation, often catalysed by acid or metal ions ${ }^{[135,136]}$. However, compared with Phormium fibres, paper deterioration is more complicated by their dependence on both the raw materials and the papermaking process ${ }^{[133,137]}$. Common external degradative factors include light, moisture, temperature, microorganisms and chemical pollutants, which often interact synergistically with each other to exacerbate the deterioration ${ }^{[135]}$. 
One form of paper deterioration that has been intensively studied is discolouration, appearing as yellowing, staining or foxing on aged books and paper. Studies on discolouration have been carried out to test various causative hypotheses ${ }^{[90,91,134-}$ 136, 138, 139]. Photodegradation is regarded as the most potent form of abiotic deterioration, leading to the yellowing of paper ${ }^{[91]}$. The mechanisms for the photoyellowing of paper are the same as for lignocellulosic materials ${ }^{[134]}$ (See Chapter 4). There are various experiments to study the effects of paper exposure to sunlight ${ }^{[90,91]}$. The most comprehensive test is the "Accelerated Weathering Chamber" in which a cyclic program can duplicate and control environmental parameters i.e. light, temperature and humidity to simulate conditions encountered in the real world ${ }^{[91]}$. However, it is more usual to study the photodegradation of paper by exposure to a laboratory UV lamp ${ }^{[140]}$.

Some studies have linked discolouration of paper with the presence of transition metal ions, particularly $\mathrm{Fe}^{3+}$, which can trigger free radical oxidation and accelerate the degradation of cellulose with the formation of conjugated carbonyl group chromophores ${ }^{[135]}$. This results in the socalled "corroded iron spots",[141]. A previous investigation by our group reported high levels of zinc oxide associated with a pinkish staining observed on an ancient map, which could have originated from contaminants in manufacture or conservation treatments ${ }^{[142]}$. Other studies have found that gelatin-sizing resulted in many European paper archives from the $19^{\text {th }}$ and $20^{\text {th }}$ centuries being in very bad condition ${ }^{[137,139,143]}$, because the alum used to precipitate the resin of the sizing can produce sulphuric acid by hydrolysis ${ }^{[144]}$.

A broad range of spectroscopic techniques including reflectance ${ }^{[135]}, \operatorname{Raman}^{[145]}$, Fourier transform infrared spectroscopy $(\mathrm{FTIR})^{[91]}$, mass spectrometry ${ }^{[132]}$, X-ray fluorescence $(\mathrm{XRF})^{[146]}$, nuclear magnetic resonance $(\mathrm{NMR})^{[147,148]}$ and $\mathrm{X}$-ray photoelectron spectroscopy (XPS) ${ }^{[143]}$ are regularly used to monitor the optical/colour change and chemical modification of paper during ageing ${ }^{[91,149]}$.

Some discolouration is caused by fungal infection and is given the name "irregular fungal fox spot" or "foxing", to describe the rusty red spots which have 
a red colour similar to that of fox fur ${ }^{[141]}$. Mycological analyses have ascribed this biogenic discolouration of paper to infection by xerophilic fungi (e.g. Aspergillus and Penicillium $)^{[138,150]}$; and the coloured products are suggested to result from reactions between paper substrates (not only cellulose) and fungal metabolites ${ }^{[138]}$. Scanning electron microscope $(\mathrm{SEM})^{[141,151,152]} \operatorname{Raman}^{[153]}, \operatorname{FTIR}^{[152,154]}$ and $\mathrm{XRF}^{[155]}$ are frequently used in exploring stained historical papers. Variations of the chemical composition of foxing, particularly the formation of some metallic compounds, are considered as important indicators of the fungal growth ${ }^{[151,152,155]}$.

Chinese handmade papers are manufactured from plants indigenous to China. Papers made from blue sandalwood (Pteroceltis tatarinowii Maxim.), mulberry (Morus alba) and paper mulberry (Broussonetia papyrifera) are valued for their whiteness and durability ${ }^{[33,38]}$. Since antiquity, paper has been produced using traditional methods, including maceration, steaming, soaking with wood ashes, pounding, natural/solar bleaching etc. ${ }^{[33]}$ (More detailed information about traditional papermaking in China is given in Chapter 1 ) Since the $20^{\text {th }}$ century, technical improvements have been made with the use of synthetic chemical agents and machine processing which significantly accelerate the manufacturing process $^{[38]}$. However, the chemically-facilitated methods of manufacture have reduced the quality of paper, particularly the lightfast performance, and concerns are raised about their appropriateness for use in paper conservation ${ }^{[38]}$.

To investigate the photostabilities of three major types of Chinese handmade paper, nine modern papers manufactured using traditional or chemicallyfacilitated methods were artificially aged by UVA radiation and the changes to their optical properties were evaluated by reflectance and fluorescence spectroscopies. Their photooxidative stresses associated with production of hydrogen peroxide and superoxide radical during irradiation were measured, and possible photooxidation mechanisms are discussed. This study is expected to provide fundamental knowledge for how raw materials and production techniques determine the photostability of Chinese handmade paper, which in turn impacts on the stability of books, artwork or other objects in which the paper is used. 


\subsection{Materials and Methods}

\section{Chinese Handmade Paper}

The three types of Chinese handmade paper investigated were Xuan paper, Mulberry paper and Bamboo paper, made mainly from blue sandalwood (Pteroceltis tatarinowii Maxim.), mulberry (Morus alba) and/or paper mulberry (Broussonetia papyrifera), and bamboo (Phyllostachys aurea), respectively. Paper samples were sourced from two reputable papermaking provinces in China, Anhui (Jing County) and Zhejiang (Wenzhou and Fuyang), where the papers were manufactured using either traditional or chemically-facilitated methods (Table 5.1). All paper samples were provided by the Department of Scientific History and Archaeometry, Graduate University of Chinese Academy of Sciences (Beijing, China).

Table 5.1 List of Chinese handmade papers studied in this thesis

\begin{tabular}{|c|c|c|c|c|c|}
\hline Type & No. & Pulping & Bleaching & Manufacturer & Origin \\
\hline \multirow{3}{*}{$\begin{array}{l}\text { Xuan } \\
\text { Paper }\end{array}$} & $\mathrm{X} 1$ & traditional & natural & Red Star & Jing County \\
\hline & $\mathrm{X} 2$ & traditional & natural & Qian-Nian-Gu-Xuan & Jing County \\
\hline & $\mathrm{X} 3$ & traditional & natural & Wang-Liu-Ji & Jing County \\
\hline \multirow{2}{*}{$\begin{array}{c}\text { Mulberry } \\
\text { Paper }\end{array}$} & M1 & chemical & natural & Qian-Nian-Gu-Xuan & Jing County \\
\hline & M2 & traditional & natural & Unknown & Wenzhou \\
\hline \multirow{4}{*}{$\begin{array}{c}\text { Bamboo } \\
\text { Paper }\end{array}$} & B1 & traditional & natural & A-De & Fuyang \\
\hline & B2 & traditional & chemical & A-De & Fuyang \\
\hline & B3 & chemical & unbleached & Xin-San-Yuan & Fuyang \\
\hline & B4 & chemical & chemical & Xin-San-Yuan & Fuyang \\
\hline
\end{tabular}




\section{Irradiation and Characterisation}

$0.2 \mathrm{~g}$ of paper samples for wet or dry UVA-irradiation were placed at a distance of $1 \mathrm{~cm}$ from two $6 \mathrm{~W}$ 'blacklight' UV fluorescent lamps (NEC FL6BL-B) with a maximum output at $366 \mathrm{~nm}$ and a spectrally integrated intensity of irradiance between 350 and $400 \mathrm{~nm}$ of $4.2 \mathrm{~mW} \cdot \mathrm{cm}^{-2}$. The spectral distribution of the radiation source was shown in Chapter 4, Figure 4.3. Wet irradiations were performed using identical methods to those described in Chapter 4.

Photoinduced yields of $\mathrm{H}_{2} \mathrm{O}_{2}$ and $\mathrm{O}_{2}^{--}$(indicated by an enhancement of $\mathrm{H}_{2} \mathrm{O}_{2}$ production in the presence of SOD) in water that was in contact with the irradiated paper samples were quantified using the xylenol colorimetric assay as described in Chapter 4. Spectroscopic characterisations including UV-Vis reflectance and fluorescence spectroscopy were also carried out using the experimental methods described in the previous chapters.

\subsection{Results and Discussion}

\subsubsection{Fluorescence of Three Traditional Papers}

Figure 5.1 shows the paper samples of the three types: Xuan Paper (A), Mulberry paper (B) and Bamboo paper (C). The first two papers were made from pounded tree bark (wood pulp), are of a brilliant white in appearance and are several metres in length. The Bamboo paper was made from bamboo twigs (non-wood pulp). The papers shown in the figure were all manufactured by traditional methods, i.e. made mostly with natural materials, hand utensils and tools, without any additional synthetic chemical agents. 


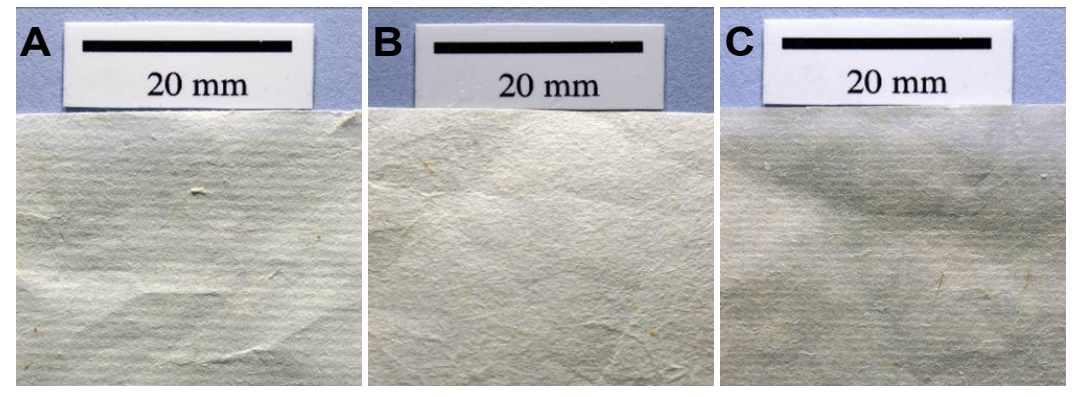

Figure 5.1 Picture of three types of traditional Chinese handmade paper: Xuan paper (A), Mulberry paper (B) and Bamboo paper (C).

Their fluorescence excitation/emission spectra and the $\mathrm{pH}$-dependent behaviours of these spectra are displayed as "3D-contour maps" in Figure 5.2, with descriptions as below:

- At neutral pHs, Xuan paper and Mulberry paper exhibited fluorescence spectra that were similar to hydroxycoumarins and other polyphenolics in the visible blue region with a maximum around $450 \mathrm{~nm}$.

- However, when the alkalinity was increased, their fluorescence excitation and emission spectra responded differently to that observed for coumarins. For Xuan paper at higher $\mathrm{pH}$, there was a substantial increase in fluorescence intensity but no observable spectral changes. This enhanced emission made it the only sample that become "optically brighter" at higher pHs. By contrast, the fluorescence of Mulberry paper decreased and slightly red shifted, which makes the paper appear duller.

- Bamboo paper displayed multiple fluorophores. At the original $\mathrm{pH}$, in addition to the major polyphenolic-like fluorophore emitting at $450 \mathrm{~nm}$, there was a second fluorescent species emitting at $500 \mathrm{~nm}$. At alkaline pHs, the fluorescence emissions red shifted with the formation of a set of three species absorbing in the blue region, which had the effect of yellowing of the sample. 
- When the acidity was increased, the fluorescence yields of all three papers decreased but to varying extents. Mulberry paper was apparently more stable under acidic conditions than the others.

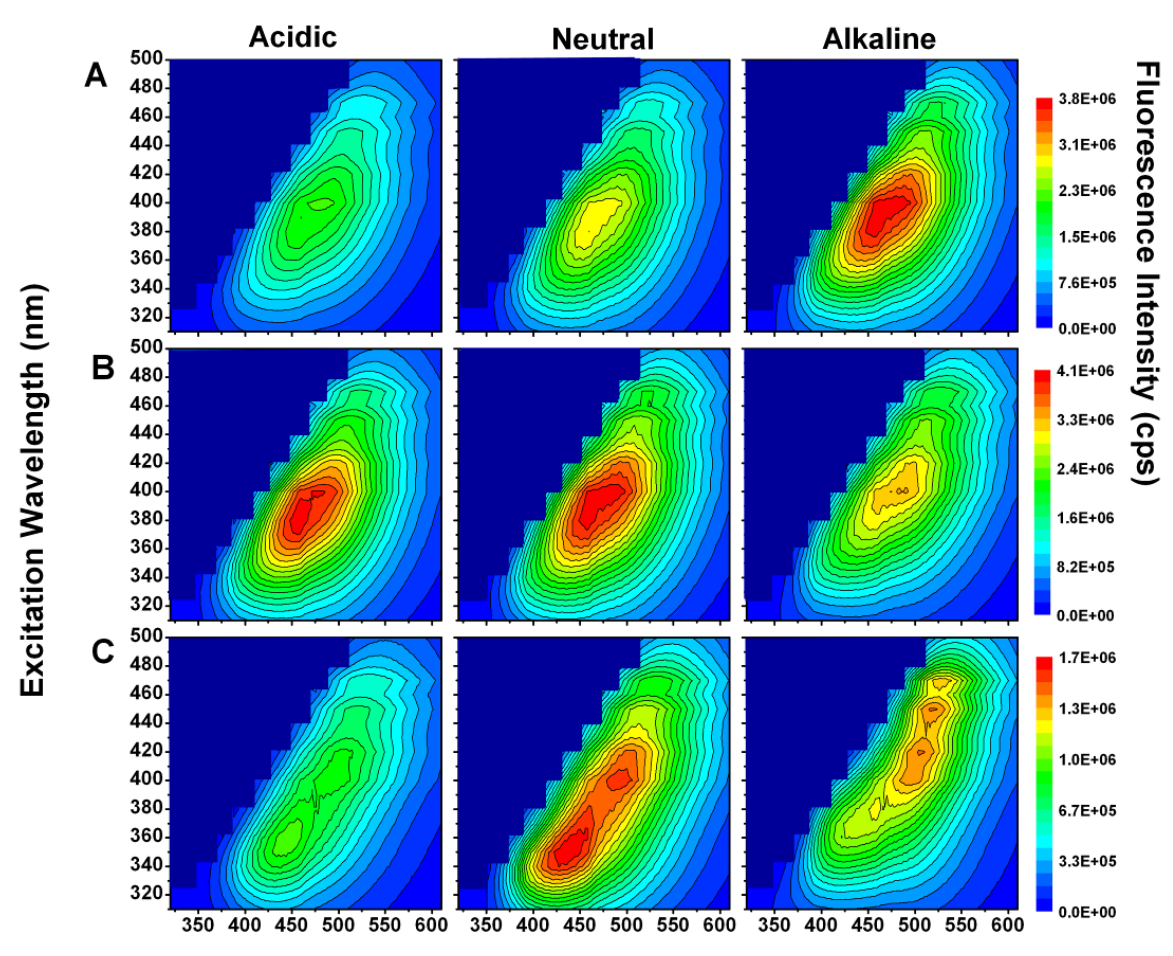

Emission Wavelength (nm)

Figure 5.2 3D-fluorescence spectra of three types of traditional Chinese handmade paper (original pHs $6.3 \sim 8.0$ ), Xuan paper (A), Mulberry paper (B) and Bamboo paper $(\mathrm{C})$, after treatments with weak acid $(\mathrm{HCl}, \mathrm{pH} 4.0)$ or base $\left(\mathrm{NaHCO}_{3}, \mathrm{pH}\right.$ 9.0).

\subsubsection{UVA-induced Spectroscopic Changes}

To further assess the quality of the three types of Chinese handmade paper, their photochemical responses to UVA radiation were examined using UV-Vis reflectance and fluorescence spectroscopies. For each type of paper, samples from various sources and/or produced from different manufacture, viz. by traditional or chemically-facilitated methods, are compared. 


\section{Xuan Paper}

Xuan paper is made from the bark of blue sandalwood (Pteroceltis tartarinowii, Maxim.) mixed with rice straw (Oryza sativa). This paper is renowned for being soft and fine textured, suitable for painting and calligraphy. In this project, UV-Vis reflectance and fluorescence spectroscopic examinations of three Xuan papers were made, labelled X1-X3, which were all manufactured traditionally in Jing County of East China, where the traditional handicrafts of making Xuan paper originated in the $7^{\text {th }}$ century and has been inscribed into the United Nations Educational, Scientific and Cultural Organization (UNESCO) "Representative List of the Intangible Cultural Heritage of Humanity" in $2009^{[156]}$.

The reflectance and 3D-fluorescence spectra of papers before and after $24 \mathrm{hrs}$ of dry/wet UVA-irradiation are shown in Figure 5.3 and Figure 5.4, respectively. The results are summarised below:

- As seen in the reflectance spectra, variations of whiteness were observed in the unirradiated samples: $\mathrm{X} 3>\mathrm{X} 2>\mathrm{X} 1$, and the intensity of their fluorescence also follow this trend.

- Exposure of all dry papers to UVA radiation resulted in shifts of the excitation/absorption bands to longer wavelengths and reductions of their reflectance across the entire visible spectrum. The photoyellowing of Xuan paper could therefore be attributed to the loss of a blue fluorophore $(\sim 400$ $-500 \mathrm{~nm}$ ) as well as the production of chromophores absorbing in the visible wavelength range.

- However, the fluorescence excitation and emission maxima of papers after wet irradiation remained unchanged as observed in the unirradiated samples. This, together with a smaller reduction in the fluorescence quantum yields, made the paper appear less yellowed when irradiated wet than dry. This implies there is a different pathway of photodegradation of the Xuan paper in an aqueous environment. 
- The relative photostabilities of the three Xuan papers when irradiated wet is: $\mathrm{X} 3>\mathrm{X} 2>\mathrm{X} 1$. This is probably because of different proportions of various raw materials used in their manufacture; as stated in ancient records, "the more the bark the better the paper"[33]. It suggested the rice straws may have higher lignin contents or contain higher levels of photosensitising trace metals that decrease the lightfastness of Xuan paper.

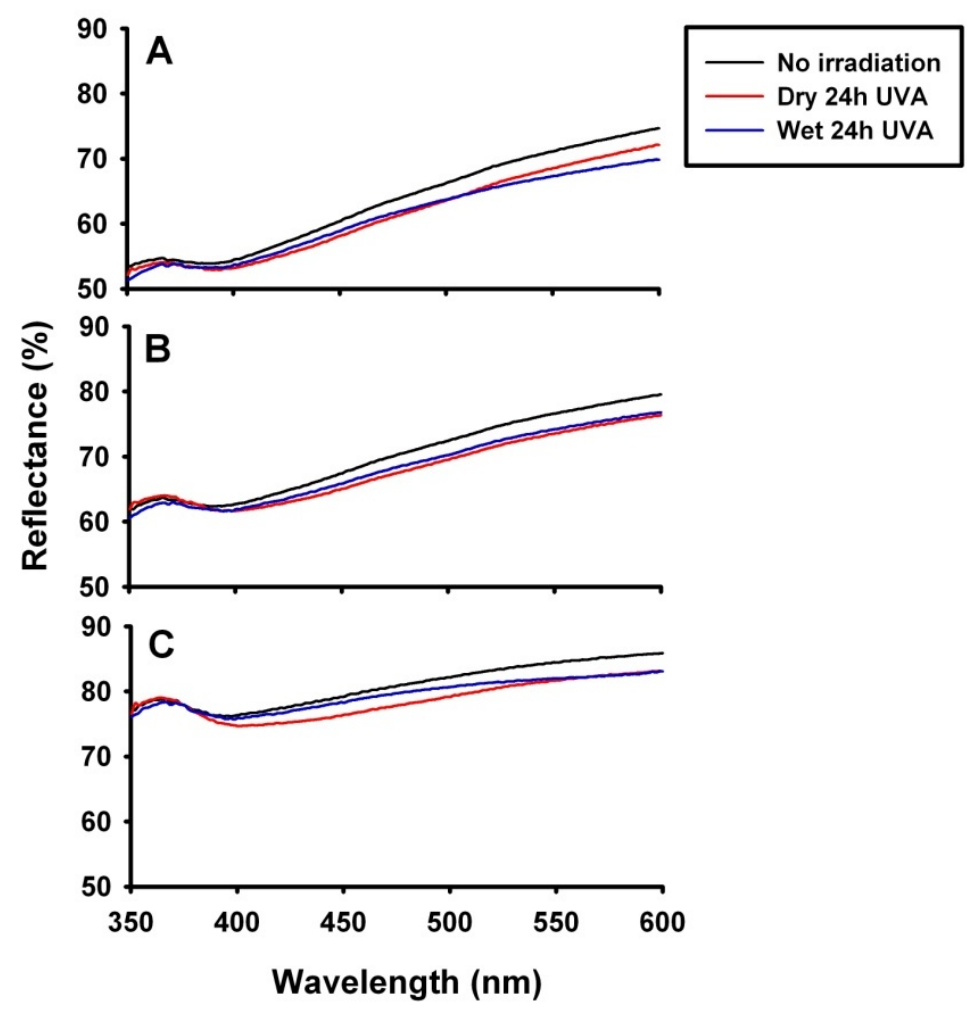

Figure 5.3 Reflectance spectra of three Xuan papers before (black curve) and after 24 hrs of UVA irradiation under dry (red curve) or wet (blue curve) conditions: X1 (A), X2 (B) and X3 (C). 


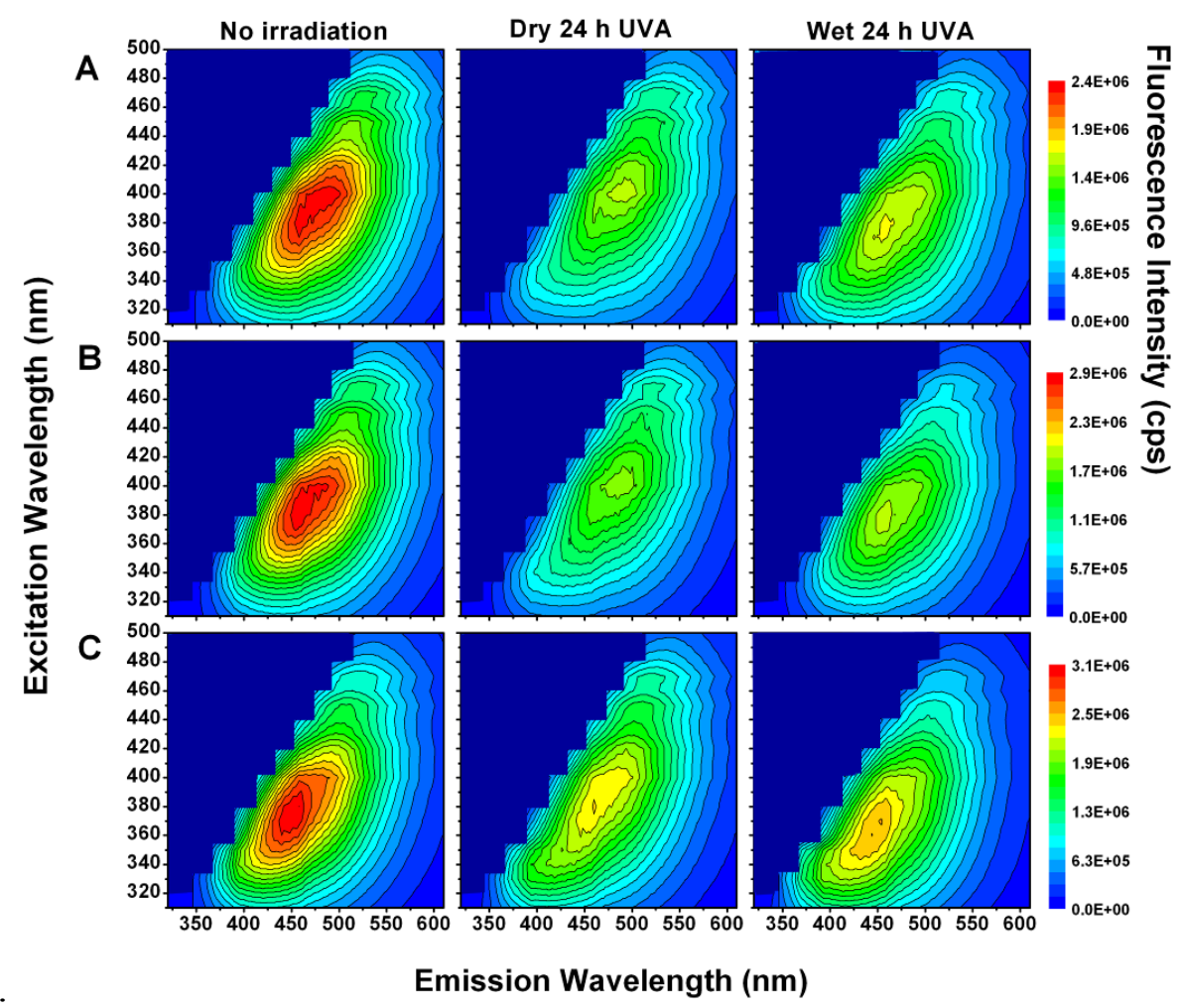

Figure 5.4 3D-fluorescence spectra of three Xuan papers before (left side) and after 24 hrs of UVA irradiation, under dry (middle) or wet (right side) conditions: X1 (A), X2 (B) and X3 (C).

\section{Mulberry Paper}

Mulberry paper is made from the bark of mulberry (Morus alba) and paper mulberry (Broussonetia papyrifera); the former was chiefly planted for the production of silk from silkworms (Bombyx mori). Besides being used for writing, Mulberry paper was historically the important paper used for paper money, book covers, clothing and decorations ${ }^{[33]}$.

In this project, studies were made on two Mulberry papers of different origins. M1 was made from chemical pulps by the same manufacturer of X1 in Jing County, while M2 was produced traditionally in Wenzhou, a coastal area of east China. Their reflectance and 3D-fluorescence spectra upon UVA irradiation are shown in 
Figure 5.5 and Figure 5.6, respectively.

- Both unirradiated papers displayed the polyphenolic-like fluorophores. However, the fluorescence excitation and emission maxima of M2 were slightly more blue-shifted than that of M1. When irradiated in the dry state, there was a gradual loss and red-shift of the fluorescence in the two paper samples, which is similar with the spectral changes of fluorescence observed in Phormium fibres (see Chapter 4, Figure 4.5).

- However, on wet irradiation, the fluorescence spectral profile of the traditional paper, M1, remained unchanged in contrast to a blue-shift of fluorescence excitation and emission maxima observed in M2, which made the paper appear less yellowed. M1 also demonstrated a substantial reduction of reflectance but mostly due to the non-fluorescing chromophores absorbing at longer wavelengths.

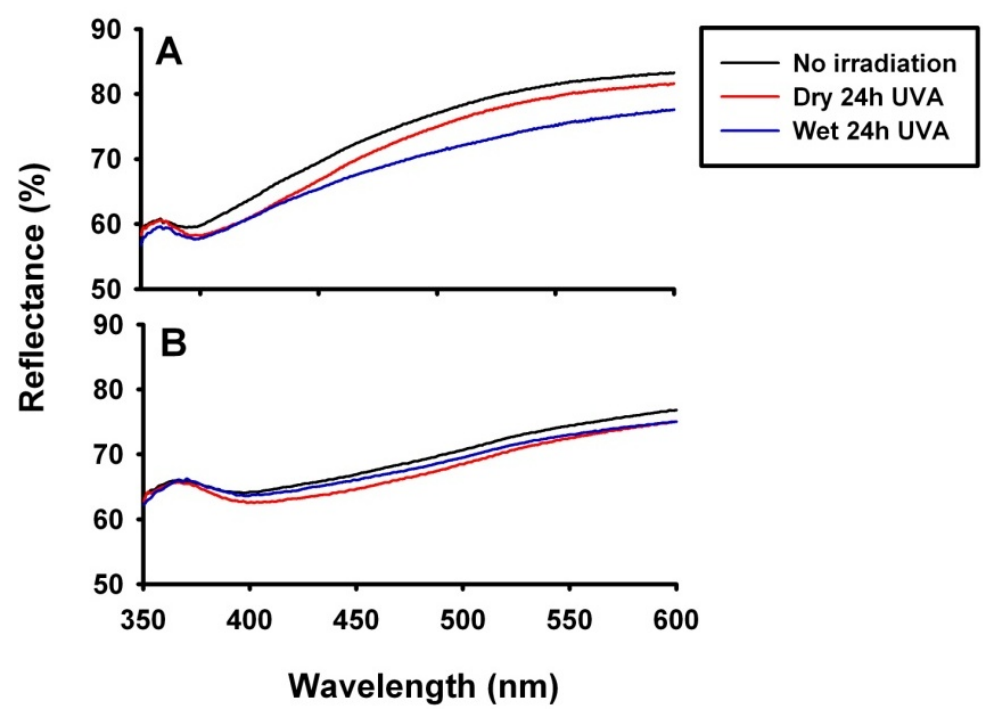

Figure 5.5 Reflectance spectra of solid fibres from two Mulberry papers, M1 (A) and M2 (B), before (black curve) and after 24 hrs of UVA irradiation under dry (red curve) or wet (blue curve) conditions. 


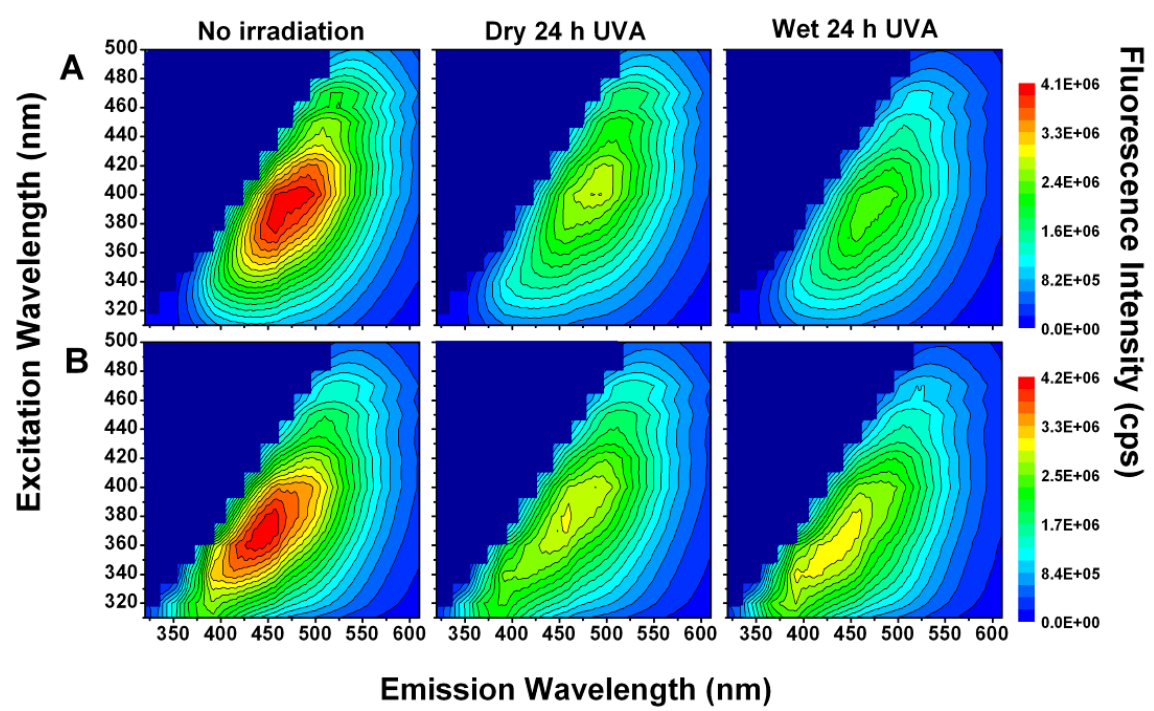

Figure 5.6 3D-fluorescence spectra of two Mulberry papers, M1 (A) and M2 (B), before (left side) and after 24 hrs of UVA irradiation, under dry (middle) or wet (right side) conditions.

\section{Bamboo Paper}

First made in the middle Tang dynasty in 8 th century and perfected in the $12^{\text {th }}$ century, Bamboo paper has long been the most popular paper, especially with $\operatorname{artists}^{[33]}$. The four paper samples of the present study, B1 - B4, are sourced from Fuyang, China, where the tradition of making Bamboo paper has been continued for hundreds of years.

Of the four samples studied, B1 and B2 were of the same origin and were both made from traditional pulps, but the latter had been chemically bleached. By contrast, B3 and B4 were produced by a different manufacturer and were made from chemical pulps. B4 had also been chemically bleached while B3 was an unbleached sample. The UV-Vis reflectance and 3D-fluorescence spectra of all four papers after $24 \mathrm{hrs}$ of UVA-irradiation are shown in Figure 5.7 and Figure 5.8, respectively. The results are discussed below: 
- Irrespective of the origin of the paper, multiple fluorophores were only apparent in the naturally-bleached and unbleached samples, B1 and B3. This is in contrast with the chemically-bleached papers that displayed only one fluorophore emitting in the blue spectral region with a maximum at $450 \mathrm{~nm}$ for $\mathrm{B} 2$ and at $475 \mathrm{~nm}$ for B4. The absence of other minor fluorophores absorbing at longer wavelengths in B2 and B4 suggests these chromophores were removed by the chemical bleaching agents.

- It is of interest to note that the spectral "fingerprint" of the multiple fluorophores of B3, the unbleached paper made from chemical pulps, was almost identical to that of the traditional paper $\mathrm{B} 1$ when its $\mathrm{pH}$ was adjusted to 9.0 (see Figure 5.2). $\mathrm{pH}$ measurements of the two untreated paper samples indicated that $\mathrm{B} 3$ had a $\mathrm{pH}$ of 7.7 , that is higher than $\mathrm{B} 1$ with a $\mathrm{pH}$ of 6.3 . This suggests that papers manufactured by chemicalpulping methods could be less acidic than traditionally pulped papers.

- On exposure to UVA radiation, the two papers made from traditional pulps, B1 and B2, displayed spectral changes similar to those observed in photolysed Xuan paper, i.e. red-shifted fluorophores in the dry state and greater fluorescence yields than when irradiated wet. The dry irradiation thereby resulted in a greater reduction in the paper's reflectance, leading to a more rapid yellowing. In this respect, B1 was more photostable than B2.

- By contrast, the photochemical behaviours of the two papers made from chemical pulps, B3 and B4, were more similar to that previously observed for Phormium fibres and Mulberry paper M1, which displayed more photodegradation with wet irradiations. B3 was more photostable than B4.

From the above analyses, it is concluded that papers produced by traditional manufacturing methods were more lightfast on UVA irradiation than other chemically-manufactured papers under both dry and wet conditions. Also, a lesser reduction in the fluorescence yields was observed in all papers derived from traditional pulps, which makes the paper more lightfast in the wet state. 


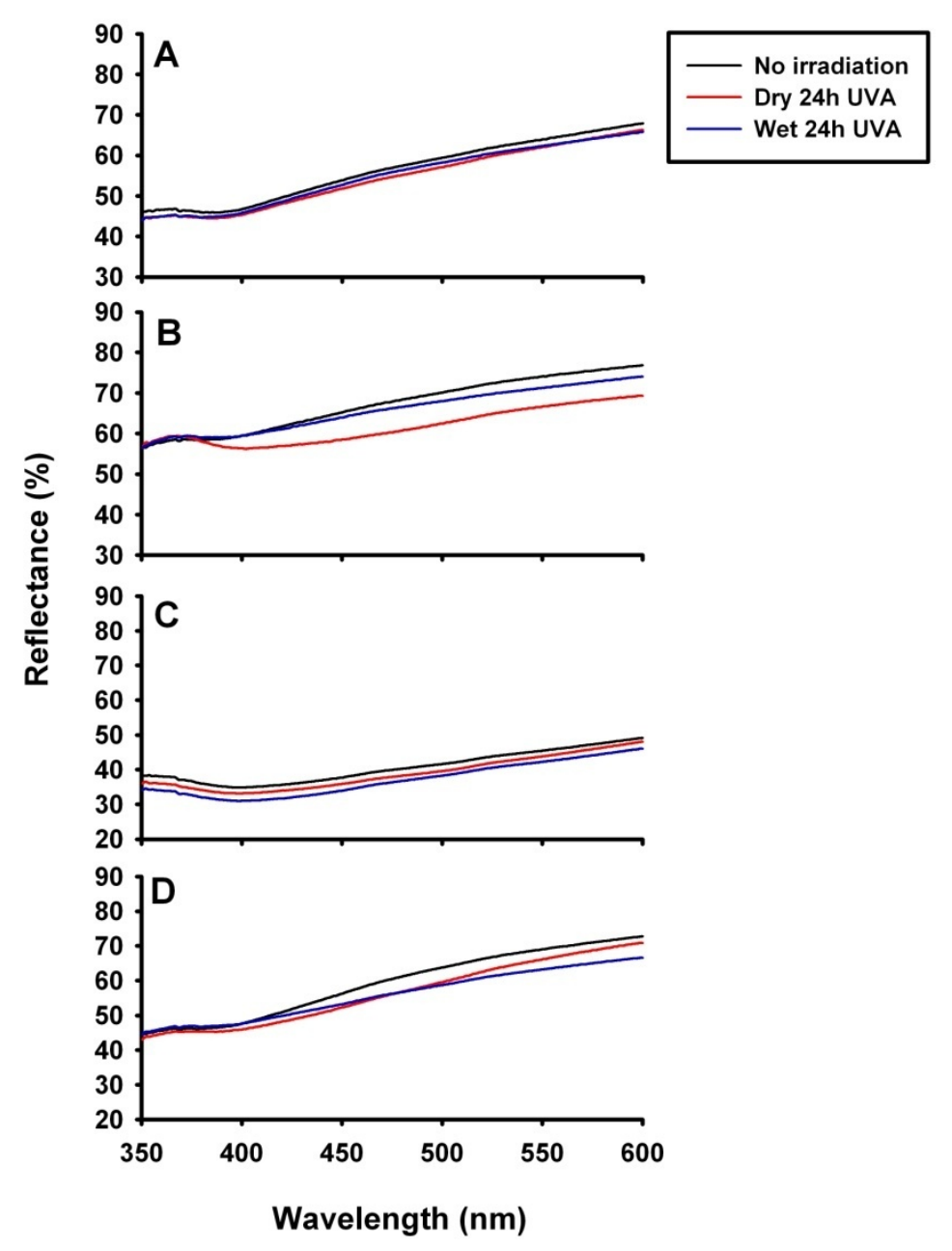

Figure 5.7 Reflectance spectra of four Bamboo papers before (black curve) and after 24 hrs of UVA irradiation under dry (red curve) or wet (blue curve) conditions: B1 (A), B2 (B), B3 (C) and B4 (D). 


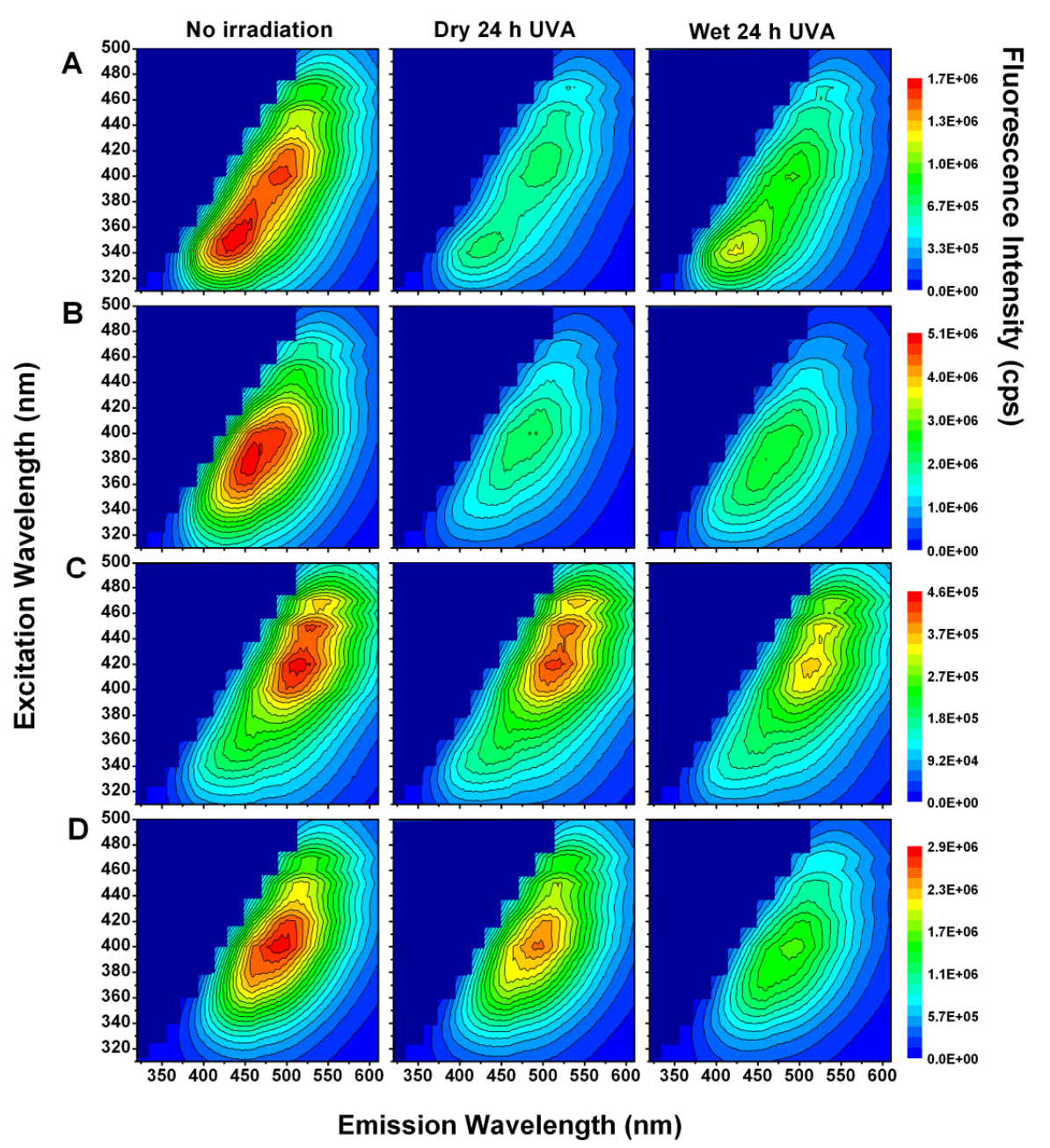

Figure 5.8 3D-fluorescence spectra of four Bamboo papers before (left side) and after 24 hrs of UVA irradiation, under dry (middle) or wet (right side) conditions: B1 (A), B2 (B), B3 (C) and B4 (D). 


\subsubsection{Generation of Peroxide and Superoxide}

The UVA-induced productions of $\mathrm{H}_{2} \mathrm{O}_{2}$ and $\mathrm{O}_{2}{ }^{-}$in the nine samples of Chinese handmade paper were measured using the xylenol colorimetric assay described in Chapter 4 after irradiation for 2, 8, 16 and $24 \mathrm{hrs}$.

\section{Xuan Paper}

The yields of $\mathrm{H}_{2} \mathrm{O}_{2}$ for the three traditional Xuan papers at irradiation times from 0 to 24 hrs are summarised in Figure 5.9. It should be noted that the amounts of $\mathrm{H}_{2} \mathrm{O}_{2}$ in all unirradiated samples are not zero $(\sim 0.5$ to $1.0 \mu \mathrm{M})$. This is possibly the result of a dark reaction or residual peroxides present in unirradiated papers.

Figure 5.9 also shows the addition of superoxide dismutase (SOD, 250 Units $\cdot \mathrm{mL}^{-1}$ ) to all irradiated samples resulted in the generation of higher levels of $\mathrm{H}_{2} \mathrm{O}_{2}$, indicating the presence of $\mathrm{O}_{2}^{--}$(see Chapter 4, Section 4.2 in all cases). After $2 \mathrm{hrs,}$ the enhanced yields of $\mathrm{H}_{2} \mathrm{O}_{2}$ decreased to a plateau at extended irradiation times. In all paper samples examined, little or no $\mathrm{H}_{2} \mathrm{O}_{2}$ was observed with the addition of catalase, a specific enzyme for $\mathrm{H}_{2} \mathrm{O}_{2}$, showing the production of $\mathrm{H}_{2} \mathrm{O}_{2}$ was derived from the photolysis of the paper.

The relative levels of $\mathrm{H}_{2} \mathrm{O}_{2}$ and $\mathrm{O}_{2}{ }^{--}$of the three paper samples, $\mathrm{X} 1>\mathrm{X} 2>\mathrm{X} 3$, could be correlated to their optical photostabilities described in the previous spectroscopic study (see Section 5.3.2). The highest yielding peroxide and superoxide paper, X1, also showed the largest spectral changes of reflectance and fluorescence when irradiated wet. This has suggested that the photosensitised formation of $\mathrm{H}_{2} \mathrm{O}_{2}$ and $\mathrm{O}_{2}{ }^{-}$is associated with the UVA-induced degradation of Xuan paper and a type II or electron transfer mechanism of photooxidation is probably involved in this process. 

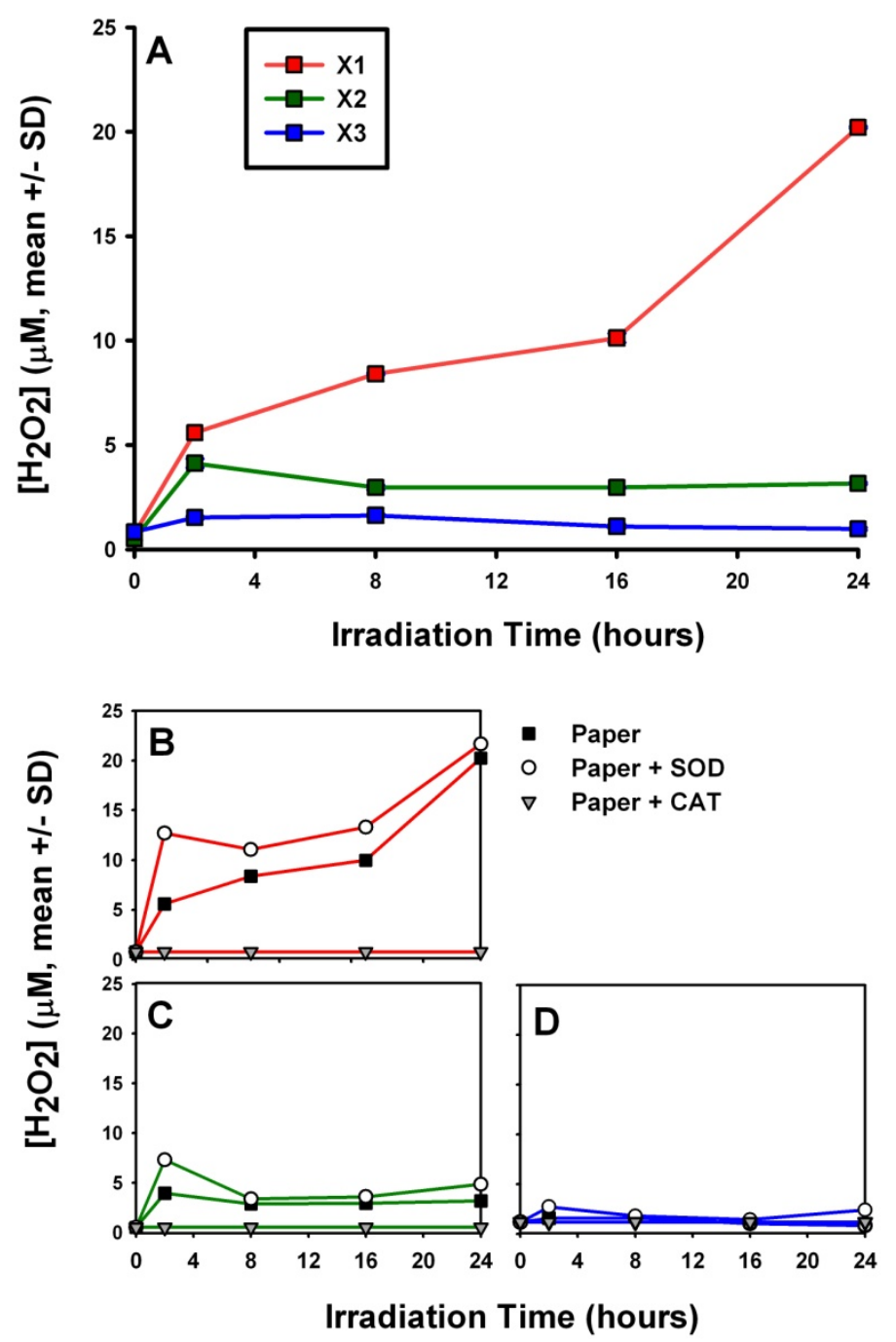

Figure 5.9 Production of $\mathrm{H}_{2} \mathrm{O}_{2}$ in three samples of wet UVA-irradiated Xuan Paper (A) and the effect of superoxide dismutase (SOD) and catalase (CAT): X1 (B), X2 (C) and $\mathrm{X} 3(\mathrm{D}) . \mathrm{H}_{2} \mathrm{O}_{2}$ is shown as concentration $(\mu \mathrm{M})$ in the water from irradiated paper over exposure time from 0 to $24 \mathrm{hrs}$. 


\section{Mulberry Paper}

Figure 5.10 compares the yields of $\mathrm{H}_{2} \mathrm{O}_{2}$ and $\mathrm{O}_{2}{ }^{-}$for two Mulberry papers made from differently manufactured pulps. It is apparent that the paper made from chemical pulps, M1, produced a higher concentration of $\mathrm{H}_{2} \mathrm{O}_{2}$ as well as an increased level of $\mathrm{H}_{2} \mathrm{O}_{2}$ in the presence of SOD than the paper made from traditional pulps, M2, during the irradiation periods. No $\mathrm{H}_{2} \mathrm{O}_{2}$ was detected with the addition of catalase. This order of $\mathrm{H}_{2} \mathrm{O}_{2}$ and $\mathrm{O}_{2}{ }^{-}$yields on exposure to UVA radiation, M1 > M2, is consistent with their optical/spectroscopic changes observed following wet irradiation (see Section 5.3.2).
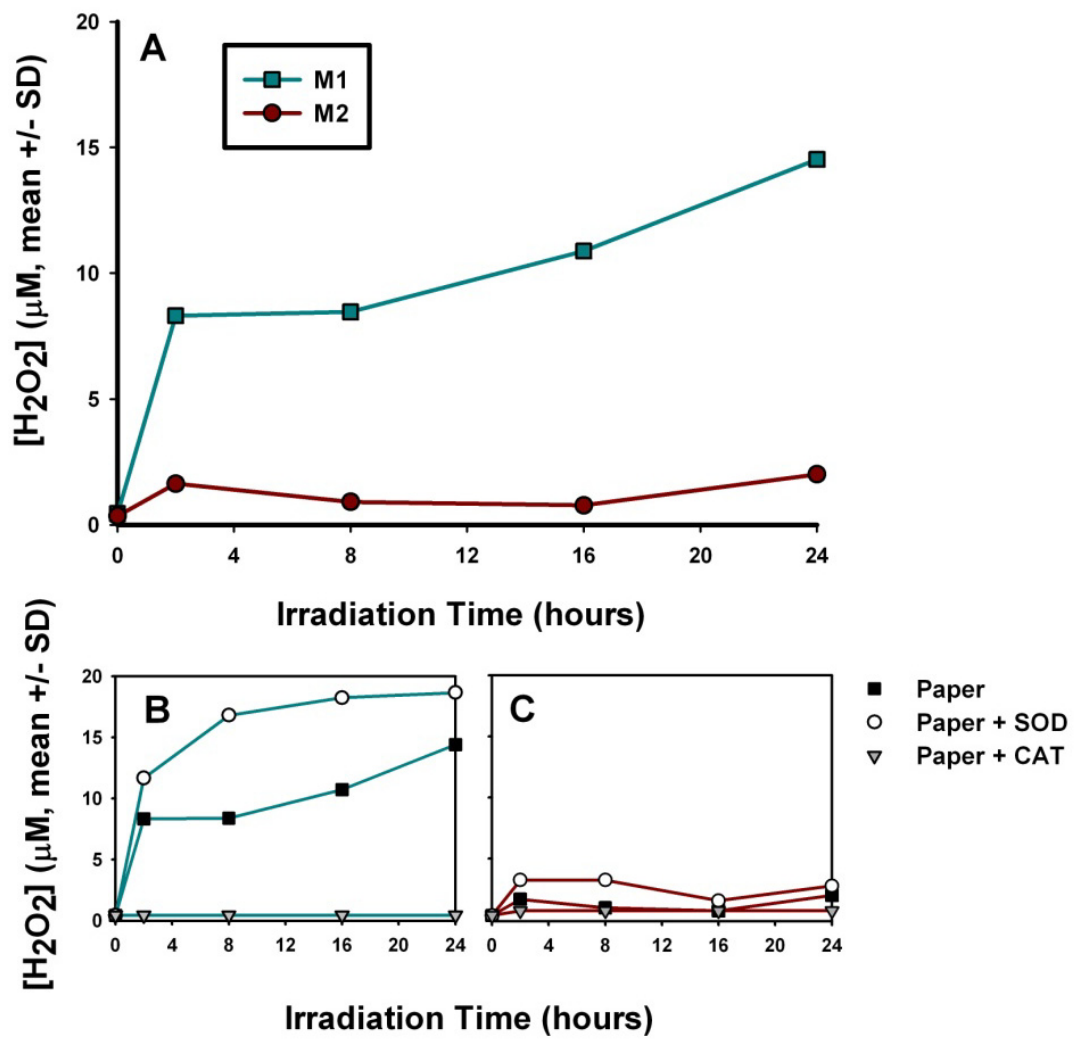

Figure 5.10 Production of $\mathrm{H}_{2} \mathrm{O}_{2}$ in two samples of wet UVA-irradiated Mulberry paper (A) and the effect of superoxide dismutase (SOD) and catalase (CAT) on $M 1(B)$ and $M 2(C) . H_{2} \mathrm{O}_{2}$ is shown as concentration $(\mu M)$ in the water from irradiated paper over exposure time from 0 to $24 \mathrm{hrs}$. 


\section{Bamboo Paper}

Figure 5.11 shows the UVA-induced generation of $\mathrm{H}_{2} \mathrm{O}_{2}$ and the effect of SOD and catalase on the four Bamboo papers made by two different manufacturers. The results are discussed below:

- The two paper samples derived from traditional pulps, B1 and B2, produced similar amounts of $\mathrm{H}_{2} \mathrm{O}_{2}$ in the first $16 \mathrm{hrs}$ of irradiation. After that, the $\mathrm{H}_{2} \mathrm{O}_{2}$ production of $\mathrm{B} 1$, the naturally-bleached paper, decreased to undetectable level, while that of B2, the chemically-bleached paper, remained constant. The addition of SOD resulted in increased levels of $\mathrm{H}_{2} \mathrm{O}_{2}$ with the maximum yields reached after $2 \mathrm{hrs}$ of irradiation, indicating the formation of $\mathrm{O}_{2}^{{ }^{-}}$.

- The highest yield of $\mathrm{H}_{2} \mathrm{O}_{2}$ measured was obtained from B3, the unbleached paper produced from chemical pulps. When many of the visible chromophores are absent in the chemically-bleached paper, as represented by $\mathrm{B} 4$, only trace amounts of $\mathrm{H}_{2} \mathrm{O}_{2}$ were detected. This suggests that the yellow chromophores, associated with lignin present in the unbleached paper contribute significantly to peroxide production. However, no/little increase in $\mathrm{H}_{2} \mathrm{O}_{2}$ production for $\mathrm{B} 3$ was observed in the presence of SOD during irradiation periods. By contrast, there was a significant increase in the yield of $\mathrm{H}_{2} \mathrm{O}_{2}$ for $\mathrm{B} 4$, which is the paper with the highest superoxide yield of the four samples studied.

- The addition of catalase to all irradiated samples produced undetectable $\mathrm{H}_{2} \mathrm{O}_{2}$, indicating that there was no other oxidising species formed during the irradiation periods.

In summary, papers made from chemical pulps produced significantly more peroxide/superoxide than those made from traditional pulps. However, the use of different bleaching method, such as exposure to sunlight or by chemical agents, caused very little difference in the yields. 

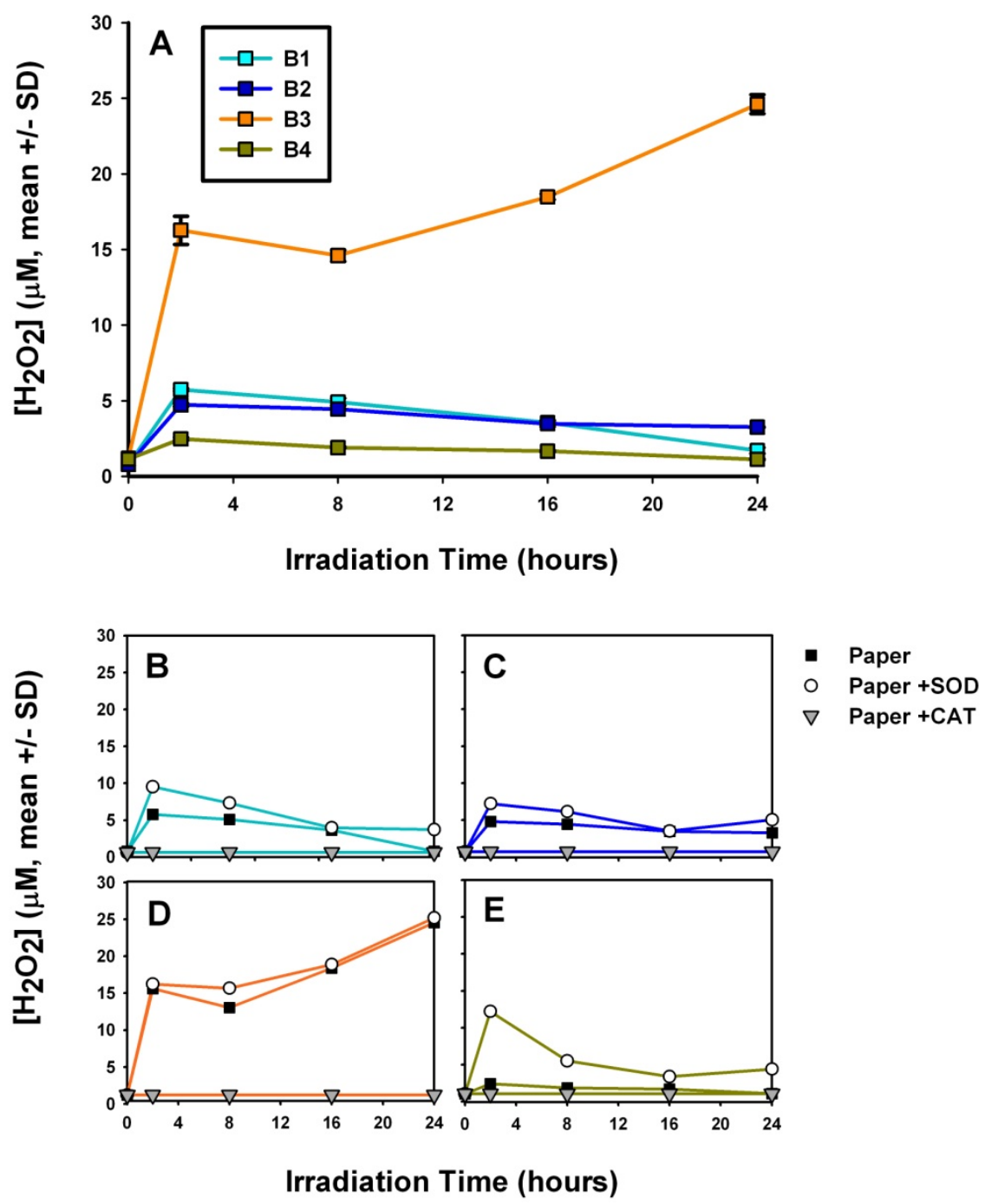

Figure 5.11 Production of $\mathrm{H}_{2} \mathrm{O}_{2}$ in four samples of wet UVA-irradiated Bamboo paper (A) and the effect of superoxide dismutase (SOD) and catalase (CAT) on B1 $(B), B 2(C), B 3(D)$ and $B 4(E) . \mathrm{H}_{2} \mathrm{O}_{2}$ is shown as concentration $(\mu M)$ in the water from irradiated paper over exposure time from 0 to $24 \mathrm{hrs}$. 


\subsection{Summary of the Chapter}

The spectroscopic and photochemical properties and behaviours of nine modern Chinese handmade papers were investigated at room temperature under UVA irradiation. This important archival material has been developed and produced in China from antiquity. The types studied in this project include Xuan paper, Mulberry paper and Bamboo paper, which are made from tree barks of blue sandalwood, mulberry and shoots of bamboo, respectively. Mostly used in calligraphy and painting, Xuan paper and Mulberry have been long valued for their whiteness, which could be attributed to their strong fluorescence in the blue region of the spectrum when excited by near UV radiation.

Like other lignocellulosic materials, exposure of Chinese handmade papers to UVA radiation resulted in decreases in their intrinsic fluorescence intensity and UV-Vis reflectance values. The photoyellowing of papers manufactured by traditional or chemical-modified processings were explored by determining the responses of various paper samples of the three major types to irradiation under both dry and wet conditions. Results showed that papers made from chemical pulps, regardless of the paper type, exhibited similar photoyellowing behaviours as previously observed in Phormium fibres (see Chapter 4). By contrast, papers produced from traditional pulps displayed an increase in the blue fluorescence when irradiated wet, which to some extent, mitigated the photoyellowing and made the traditional papers more photostable. This is the first report to date that UV irradiation of lignocellulosic materials in the wet state causing less discolouration than irradiation in the dry state.

The photosensitised formation of hydrogen peroxide and superoxide was detected in all Chinese handmade papers examined in this work. The relative yields of various papers during the irradiation are found to be dependent on the manufacturing techniques, which in turn are associated with their different yellowing rates. Papers manufactured by chemically-facilitated methods generally 
yield more hydrogen peroxide or superoxide and are thereby more subject to photooxidative yellowing.

Thus, the influence of different manufacturing techniques on the photostability of Chinese handmade paper can be summarised as follows: traditional pulp + natural bleach $>$ traditional pulp + chemical bleach $>$ traditional pulp + unbleached $>$ chemical pulp/bleached. For papers that were claimed "to be traditionally produced", i.e. the three Xuan papers of this study, their differences in lightfastness could be due to the various amounts of lignin and trace metal ions from the raw materials. To further investigate this, a scanning electron microscopic study and elemental analysis was carried out and is reported in Chapter 6. 


\section{Chapter 6}

\section{Microstructural and Elemental Analysis}

This chapter is devoted to an investigation of the microstructure, morphology and elemental composition of Phormium fibres and traditional Chinese handmade papers. The results are presented as four case studies, dealing with four examples of fibrous materials containing metal ions.

\subsection{Metal Ions on Textiles: History and Science}

\section{Mordanting Agents for Natural Dyes}

Many natural dyes (e.g. polyphenolics) require auxiliary binding agents, known as mordants to improve their colour intensity and fastness. Metal ion mordants can form a strong bond with the ionisable functional groups (e.g. $-\mathrm{OH},-\mathrm{NH}_{2}$ and $\mathrm{COOH})$ of textile fibres to provide cationic metal ion binding/linking sites for polyphenolic dyes. Mordants can be applied before, with or after dyeing, depending on the chemical nature of the dye, the fibre and the mordant. A range of colours can be obtained by mordanting a single type of dye molecule with different mordants ${ }^{[157]}$.

Since antiquity, substances from various natural sources, both mineral and biological, have been used as mordants. However, the essential ingredients in 
most historic recipes are a limited number of metal ions, such as aluminium $\left(\mathrm{Al}^{3+}\right)$, ferric $\left(\mathrm{Fe}^{3+}\right)$, cupric $\left(\mathrm{Cu}^{2+}\right)$, stannic $\left(\mathrm{Sn}^{4+}\right)$ and chromium $\left(\mathrm{Cr}^{3+}\right)$ ions. Most of these metal ions exist as salts in nature, and their usages are highly dependent on their availability. Investigations have been undertaken on the colour characterisation of natural dyeing and the effects of mordant metal ions on the optical spectroscopic properties ${ }^{[158-161]}$. Some are discussed below.

Aluminium is the most abundant metal in the earth's crust ( $8.3 \%$ by weight), and this name is derived from alum, the most widely used mordant. The most common form of mineral alum is potash alum, $\mathrm{KAl}\left(\mathrm{SO}_{4}\right)_{2} \cdot 12\left(\mathrm{H}_{2} \mathrm{O}\right)$, usually found in deserts and volcanic areas ${ }^{[162]}$. Aluminium mineral ions can also be obtained from biological sources ("vegetable alum") in places where alum is either unavailable or costly. Some clubmoss are super-accumulators of aluminium ions and are even preferred as mordants by some local dyers ${ }^{[163]}$. Traditionally, they are all described as "alum" and are used without distinguishing the source. They do, however, result in subtle variations in the hue of the dyed textiles. In a study on alizarindyed wool, different mineral alums (e.g. potash alum, ammonium alum or alunogen) gave similar colours, whereas the use of British clubmoss (Diphasium alpinum) caused the fabrics to appear paler and yellower ${ }^{[164]}$.

Iron and copper mordants were used to darken the tone of natural dyes. Ferric ions have been used in combination with tannin to produce grey or black (iron-gall ink) colours in history. Being cheap and easy to produce, iron salts (ferrous sulphate, ferrous acetate, etc.) were excavated from weathering products of white iron pyrites (iron disulphide, $\mathrm{FeS}_{2}$ ) centuries before alum ${ }^{[162]}$. The use of copper mordant began at nearly the same time as iron, as mineral copper sulphate was a by-product of ferrous sulphate manufacture from pyrites ${ }^{[162]}$.

Tin is commonly used in the form of stannous chloride $\left(\mathrm{SnCl}_{2}\right)$ to produce bright colours with many red and yellow dyes. Medieval dyers in Europe have used tin or tin-lined pot as a mordanting process ${ }^{[162]}$. Tin and chromium mordants are widely used for dyeing wool for excellent light and washfastness in spite of the health hazards caused by the high toxicity of these metals ${ }^{[162]}$. 


\section{Effects on Deterioration}

Most transition metals have rich coordination and redox chemistries which are closely linked with the generation of various free radicals. Metal ion-catalyzed processes (Fenton reaction) are involved in the major mechanisms of oxygen activation under stress conditions (e.g. UV, heat). In the absence of chelators, the Fenton reaction is driven by ferric, cupric, chromium, titanium or cobalt ions to produce reactive hydroxyl radicals $\left(\mathrm{OH}^{*}\right)^{[165]}$.

$$
\begin{gathered}
\mathrm{M}^{(\mathrm{n}+1)+}+\mathrm{O}_{2}^{{ }^{-}} \rightarrow \mathrm{M}^{\mathrm{n}+}+\mathrm{O}_{2} \\
\mathrm{M}^{\mathrm{n}+}+\mathrm{H}_{2} \mathrm{O}_{2} \rightarrow \mathrm{M}^{(\mathrm{n}+1)^{+}}+\mathrm{OH}^{\cdot}+\mathrm{OH}^{-}
\end{gathered}
$$

$\mathrm{OH}^{*}$ is the strongest oxidising species that can be produced in an aqueous environment, and will rapidly react with virtually all organic substrates. Studies with various model compounds have indicated that the attack of $\mathrm{OH}^{*}$ on subunits of lignin can produce reactive phenoxyl radical intermediates that are largely responsible for oxidative damage of lignin-containing materials ${ }^{[166]}$. Therefore, it is not surprising to find that transition metal ions, particularly $\mathrm{Fe}^{3+}$ and $\mathrm{Cu}^{2+}$, are frequently reported to be associated with yellowing, foxing, tendering and other deterioration of textiles and paper materials ${ }^{[85,141,167,168]}$.

When colourants are applied to the fibrous materials, the rate of deterioration can be altered by the dye-fibre, metal-fibre and/or dye-metal interactions ${ }^{[169]}$. Investigations have shown that the phototendering of wool can be exacerbated by the addition of polyphenolic dyes, compared with undyed wool or wool treated with metal ions alone, and different metal ions have different influences on the tendering rate of dyed wool ${ }^{[89,170]}$. Another investigation on an anthraquinone dye found pretreatment with $\mathrm{Fe}^{3+}, \mathrm{Cu}^{2+}$ and $\mathrm{Al}^{3+}$ promoted lightfastness of cotton and silk ${ }^{[171]}$. $\mathrm{Al}^{3+}$ is also reported to provide some photoprotection against the phototendering of wool, which may have been a reason for it being favoured as a mordant by dyers for thousands of years ${ }^{[85]}$. 


\section{Natural dyes and Mordants used on Phormium Fibres}

In New Zealand, the traditional dyes used by the Maori can be grouped into three colours: black, brown and yellow. The mordants used are usually metal ions alone or in association with tannin, which significantly modify the hue of the dye. Table 6.1 summerises the principal natural dyes and mordants used on Phormium fibres.

Table 6.1 Natural dyes used on Phormium textiles by Maori ${ }^{[8,25,172]}$

\begin{tabular}{ccccc}
\hline \multirow{2}{*}{ Colour } & \multicolumn{2}{c}{ Dye } & \multicolumn{2}{c}{ Mordants } \\
\cline { 2 - 5 } & Component & Source & Component & Source \\
\hline Bluish-black & Iron-tannate & ${ }^{* * *}$ Paru & Gallotannin & $\begin{array}{c}{ }^{*} \text { Hinau or } \\
\text { Pokaka }\end{array}$ \\
Greenish-black & & & Condensed tannin & *Manuka \\
\hline $\begin{array}{c}\text { Reddish brown } \\
\text { Pale tan }\end{array}$ & Polyphenolic & ${ }^{*}$ Tanekaha & $\mathrm{K}_{2} \mathrm{CO}_{3}$ & ${ }^{* *}$ Mahoe \\
\hline Brownish-yellow & Flavonoid & ${ }^{*}$ Puriri & Polyphenol & *Tanekaha \\
Yellow & Anthraquinone & ${ }^{*}$ Raurekau & & \\
\hline
\end{tabular}

*Bark: Tanekaha (Phyllocladus trichomanoides), Raurekau (Coprosma auredala species), Puriri (Vitex lucens), Hinau (Elaeocarpus dentatus), Pokaka (Elaeocarpus hookerianus), Manuka (Leptospermum scoparium Forst.); ${ }^{* *}$ Wood ash: Mahoe (Melicytus ramiflorus) ${ }^{* * *}$ Mud: Paru ( iron-containing mud).

The black iron-tannate dye, derived from the ferruginous swamp mud with decaying plant materials rich in tannins was perhaps the first mordant used in dyeing history and was used by many civilisations including Maori. The use of vegetable tannins of two different sources can modify the tone from bluish to greenish black. However, this iron-tannate dye is notorious for decomposing cellulosic substrates by iron-initiated free radical reactions. One stabilisation method is to treat the dyed textiles with excess ferric ion-chelators such as gallotannin or phytic acid ${ }^{[24]}$ to avoid the free radicals produced by the free ferric ions interacting with the fibrous substrates. 


\section{Paper Chemical Analysis, Morphology and Conservation}

As a major archiving medium, paper is regarded as an important cultural heritage material. Much scientific research has been dedicated to the characterisation and identification of the plant fibres and colourants that have been used in archaeological paper samples ${ }^{[39-43]}$.

A recently introduced approach to fibre identification is phytolith analysis. Phytoliths are deposits of silicon and calcium precipitated within certain plants that take up these elements from soil ${ }^{[173]}$. The occurrence and shape of such phytoliths depend on the species of plants, and plants may be identified by the shape and type of the phytoliths they produce ${ }^{[174]}$. In a recent study on modern Chinese handmade papers, silica phytoliths characteristic of rice straws were found both in the raw grass and in the manufactured Xuan paper ${ }^{[44]}$, showing that the morphological integrity of the phytoliths in rice straw is retained through the papermaking process.

Metal ions present in paper can also derive from the fillers used, sizing agents, dyes, inorganic pigments, and contaminants from papermaking or conservation treatments. Evaluation of their composition and distribution contributes to an understanding of the technology of manufacture and the establishment of the chemical and physical features of the paper necessary for deciding on treatments to conserve or restore paper-based objects ${ }^{[135,175]}$.

Approaches for paper conservation are often based on the removal of degradative agents such as excess iron, acid and microorganisms in order to diminish the destruction of cellulose, hemicellulose and lignin content of paper. For example, iron-complexing agents are introduced to reduce the iron-gall ink corrosion encountered in ancient manuscripts ${ }^{[176]}$. Also, efforts have been made to synthesise nano-particles of calcium hydroxide and magnesium hydroxide as protective coatings for paper, because the dispersions of these nano-particles are efficient in deacidification and can further form an alkaline calcium carbonate reservoir on the paper fibres by reactions with the atmospheric carbon dioxide ${ }^{[177]}$. 


\subsection{Instrumental Characterisations}

Except for atomic absorption spectrometry, most methods of chemical and microstructural analyses are used in a complementary way. Scanning electron microscopy (SEM) and the associated energy-dispersive X-ray spectroscopy (EDS) give information on the spatial distribution of elements on a micron scale, while $\mathrm{X}$-ray fluorescence (XRF) and inductively coupled plasma mass spectrometry (ICP-MS) provide the total elemental composition of the whole material/sample.

\subsubsection{Scanning Electron Microscopy (SEM)}

The scanning electron microscope (SEM) permits non-destructive microstructural characterisation, analysis and spatial distribution of elements in various heterogeneous materials. In the SEM, the object to be examined is exposed to a focused electron beam scanning in a raster fashion across the surface of the specimen. As a result of electron bombardment, secondary electrons, backscattered electrons and X-rays with energies characteristic of various elements are generated. They carry information characteristic of the material, such as surface topography, crystallinity, and elemental composition ${ }^{[178]}$.

- Secondary electrons are ejected from the atoms occupying the top surface of the specimen and produce the second electron image (SEI) showing the surface topography of the sample. Since the secondary electron emission is confined to a very small volume near the beam impact area, high resolution images can be obtained approximating the size of the focused electron beam $(\sim 5 \mathrm{~nm})^{[178]}$. Figure 6.1 shows the SEI images of four natural fibres: wool, silk, cotton and Phormium, which provide important morphological information for fibre identification. 

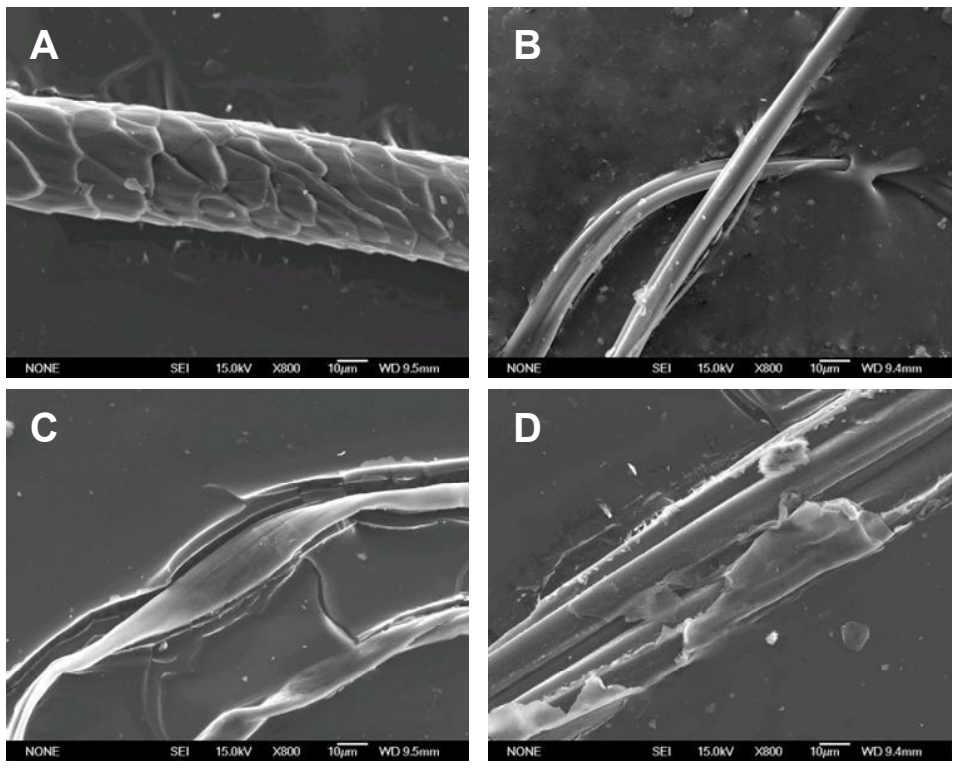

Figure 6.1 SEl images of wool (A), raw silk (B), cotton (C) and Phormium fibre (D), magnification: $\times 800$.

- Backscattered electrons are the primary beam electrons which are 'reflected' from atoms of the sample. The contrast in a backscatter image (BS) is determined by the atomic number of the elements, thus indicating the difference of chemical/atomic species distributed in the sample. Since these electrons are emitted from relatively deep in the sample, the spatial resolution of BS is not as great as SEI.

- X-ray radiation characteristic of a specific element/atom results from the electron shell transitions in atoms over regions of nominally $1 \mu \mathrm{m}$ in diameter and $1 \mu \mathrm{m}$ in depth. The measurement of X-ray energies by an energy dispersive X-ray spectrometer (EDS) permits qualitative identification and semi-quantitative analysis of elemental composition with a depth of $1-2 \mu \mathrm{m}$ into the sample. EDS can also produce elemental distribution maps or images, showing the elemental spatial distribution at the sample surface. 
The samples described in this chapter were sputter-coated with carbon to prevent electrostatic charging and then examined by a high-resolution JEOL 6500F SEM (JEOL Ltd., Tokyo, Japan) with an attached EDS stage for elemental analysis. SEI and backscatter images, elemental maps and spectra were obtained under 15 $\mathrm{kV}$ electron beam accelerating voltage and a $0^{\circ}$ sample tilt angle.

\subsubsection{X-ray Fluorescence Spectroscopy (XRF)}

$\mathrm{X}$-ray fluorescence (XRF) spectroscopy is a technique capable of non-invasively analysing the elemental composition of an object by measuring the emission of characteristic XRF of component atoms. XRF results from the electron shell transitions from higher orbitals to the innermost orbital ( $\mathrm{K}$ shell), in which an electron has been ejected by a primary excitation source of sufficient energy. Designations, alpha $(\alpha)$, beta $(\beta)$ and gamma $(\gamma)$, are used to denote transitions of electrons from higher orbitals (shells L, M, N etc.) to the K shell ${ }^{[179]}$. Since within each shell there are multiple levels of higher and lower binding energy electrons, a further assignment of $\alpha 1, \alpha 2$ or $\beta 1, \beta 2$, etc. is made to indicate transitions derived from different energy levels of these orbitals ${ }^{[179]}$.

In this project, the XRF analyses were carried out using an Amptek "X-123" spectrometer (Amptek Inc., Bedford, MA) with a lead shielded $1 \mu \mathrm{C}{ }^{241} \mathrm{Am}$ gamma ray source from a domestic smoke detector, which allows in situ inspection on various samples. The XRF spectra were interpreted using an Amptek X-ray emission K \& L line "lookup" chart ${ }^{[180]}$.

\subsubsection{Flame Atomic Absorption Spectroscopy (FAAS)}

Flame atomic absorption spectroscopy (FAAS) is an analytical technique making use of absorption of radiant energy (light) by free atoms for identifying trace concentrations of elements in acid-digested samples. Analytes are vaporised and dissociated with a high-temperature source such as a flame into hot gases of free atoms in which only individual, discrete electronic transitions are possible. Since 
the excitation energy of a particular atom corresponds to that particular electronic transition, only a defined quantity of energy i.e. a wavelength is absorbed in the continuous excitation light beam, which gives the technique its element selectivity. The amount of light absorbed (absorbance) is measured and used to determine the concentration of the element of interest in a sample (Beer-Lambert Law). FAAS is frequently used when a single metal ion is to be determined on a routine basis. Quantitative determinations are usually carried out using a calibration curve for the instrument determined using standards of known concentration of the metal ion in solution to be measured ${ }^{[181]}$.

In the first case study of this chapter, the iron content of Phormium fibres was examined by a Varian SpectrAA-400 flame atomic absorption spectrometer (Varian Inc., Mulgrave, Australia) at a wavelength of $248.3 \mathrm{~nm}$ which is one of the absorption lines of the iron atom. Samples were prepared by ashing $4 \mathrm{~g}$ (dry weight) of fibres in an electric furnace at $470{ }^{\circ} \mathrm{C}$ for $12 \mathrm{hrs}$. The resultant ashes were digested in $1 \mathrm{~mL}$ of hydrochloric acid $(6 \mathrm{M})$ with 5 drops (ca. $0.24 \mathrm{~mL}$ ) of concentrated nitric acid. The liquid mixture was then membrane-filtered and the filtrate was further diluted with DDW to make the concentration of the analyte within the range of iron standards $(1-9 \mathrm{ppm})$.

\subsubsection{ICP-Mass Spectrometry (ICP-MS)}

Inductively coupled plasma - mass spectrometry (ICP-MS) is based on combining an inductively coupled plasma, as a method of producing ions (ionisation), with a mass spectrometer as a method of separating and detecting the ions. ICP-MS offers extremely high sensitivity for a wide range of elements, particularly metals. The method has been well detailed and documented elsewhere ${ }^{[182]}$.

In the second case study, the elemental contents of wood ash were analysed with an Agilent 7500cs ICP-MS (Agilent Technologies, Tokyo, Japan). A scan including the elements $\mathrm{Mg}, \mathrm{Al}, \mathrm{Ca}, \mathrm{Fe}, \mathrm{Cu}, \mathrm{Zn}, \mathrm{Y}, \mathrm{Sn}$, and $\mathrm{Ce}$ was carried out but only the elements detected are reported in the results. 


\subsection{Results and Discussion: Four Case Studies}

\subsubsection{Determining Iron Contents of Phormium Fibres}

In Chapter 4, Phormium fibres of five cultivars, Arawa, Mawaru, Ngaro, Potaka and Tapamangu, were reported to give similar levels of $\mathrm{O}_{2}^{-}$. However, their generation of $\mathrm{H}_{2} \mathrm{O}_{2}$ varied greatly. The Fenton reaction involving ferric ions was proposed to account for the differences and to test this proposal the iron contents of the five cultivars were quantitatively determined in this study by atomic absorption spectroscopy. Fibre samples $(4.0 \mathrm{~g})$ were dry ashed at $470{ }^{\circ} \mathrm{C}$ for 12 hrs to concentrate the inorganic components. The iron concentration of each sample was calculated and plotted onto a calibration curve of standards as shown in Figure 6.2.

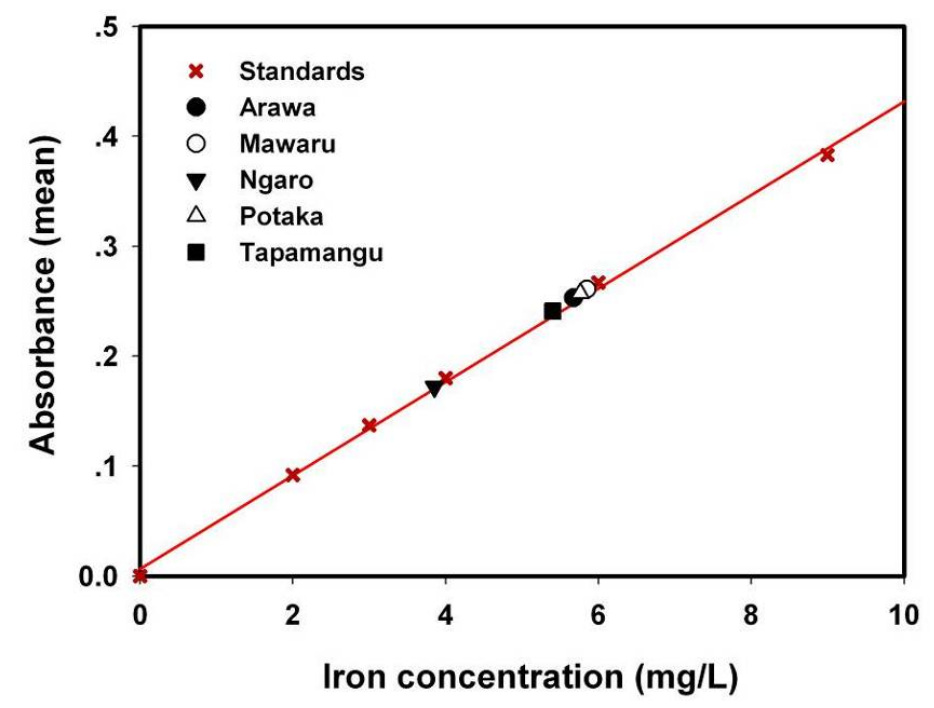

Figure 6.2 Calibration curve of iron standards and plots of sample elutions.

Table 6.2 summerises the quantified concentrations, volume of elution, precision of measurements and iron contents (ppm) of five tested samples. A relatively high 
level of iron was detected in the cultivar of Mawaru (29.25 ppm), which contains almost twice the average amount of the other cultivars.

Table 6.2 Determination of iron content of Phormium fibres from five cultivars

\begin{tabular}{|c|c|c|c|c|c|c|}
\hline P. tenax & $\begin{array}{c}\text { Fibre } \\
(\mathrm{g})\end{array}$ & $\begin{array}{l}\text { Ash } \\
(\%)\end{array}$ & $\begin{array}{c}\begin{array}{c}\text { Volume } \\
(\mathrm{mL})\end{array} \\
\end{array}$ & $\begin{array}{c}\text { Conce } \\
\left(\mathrm{mg} \mathrm{L}^{-1}\right)\end{array}$ & $\begin{array}{l}\text { ation* } \\
\text { RSD (\%) }\end{array}$ & $\begin{array}{c}\text { Iron } \\
\text { (ppm) }\end{array}$ \\
\hline Arawa & 4 & 1.23 & 10 & 5.67 & 1.72 & 14.18 \\
\hline Mawaru & 4 & 1.51 & 20 & 5.85 & 1.29 & 29.25 \\
\hline Ngaro & 4 & 0.88 & 10 & 3.85 & 2.16 & 9.63 \\
\hline Potaka & 4 & 1.39 & 11 & 5.76 & 3.25 & 15.84 \\
\hline Tapamangu & 4 & 0.84 & 10 & 5.40 & 2.64 & 13.50 \\
\hline
\end{tabular}

* Concentration is shown as the mean of three determinations

In biological systems, iron is of crucial importance in electron transfer and in the activation of molecular oxygen. Iron also plays a crucial role in oxidative stress via the Fenton-associated metal redox-cycling mechanism ${ }^{[165]}$.

$$
\begin{gathered}
\mathrm{Fe}^{3+}+\mathrm{O}_{2}^{--} \rightarrow \mathrm{Fe}^{2+}+\mathrm{O}_{2} \\
\mathrm{Fe}^{2+}+\mathrm{H}_{2} \mathrm{O}_{2} \rightarrow \mathrm{Fe}^{3+}+\mathrm{OH}^{\cdot}+\mathrm{OH}^{-}
\end{gathered}
$$

The overall reaction is the "Haber-Weiss" cycle, which only occurs in presence of enough free iron catalysts ${ }^{[99]}$.

$$
\mathrm{O}_{2}^{--}+\mathrm{H}_{2} \mathrm{O}_{2} \rightarrow \mathrm{OH}^{\bullet}+\mathrm{OH}^{-}+\mathrm{O}_{2}
$$

Under plant stress conditions (e.g. UV or fungal attack), $\mathrm{O}_{2}{ }^{-}$can release free $\mathrm{Fe}^{2+}$ ions from iron-sequestering biological molecules, which promote the yields of highly reactive hydroxyl radicals $\left(\mathrm{OH}^{\circ}\right)$ and other ROS through Fenton and/or Fenton-like reactions, thus stimulating oxidative damages ${ }^{[183,184]}$. In the present case, Figure 6.3 shows an inverse association between the iron levels of fibres from the five cultivars and their production of $\mathrm{H}_{2} \mathrm{O}_{2}$ upon UVA-irradiation. Mawaru, the cultivar with the highest iron content, also produces more $\mathrm{O}_{2}{ }^{-}$as evident in Chapter 4, Figure 4.9, indicating the "unusual" low levels of $\mathrm{H}_{2} \mathrm{O}_{2}$ of 
which could have resulted from the conversion to $\mathrm{OH}^{*}$ by iron-catalyzed Fenton reaction with the high levels of $\mathrm{O}_{2}{ }^{-}$functioning as the driving force.

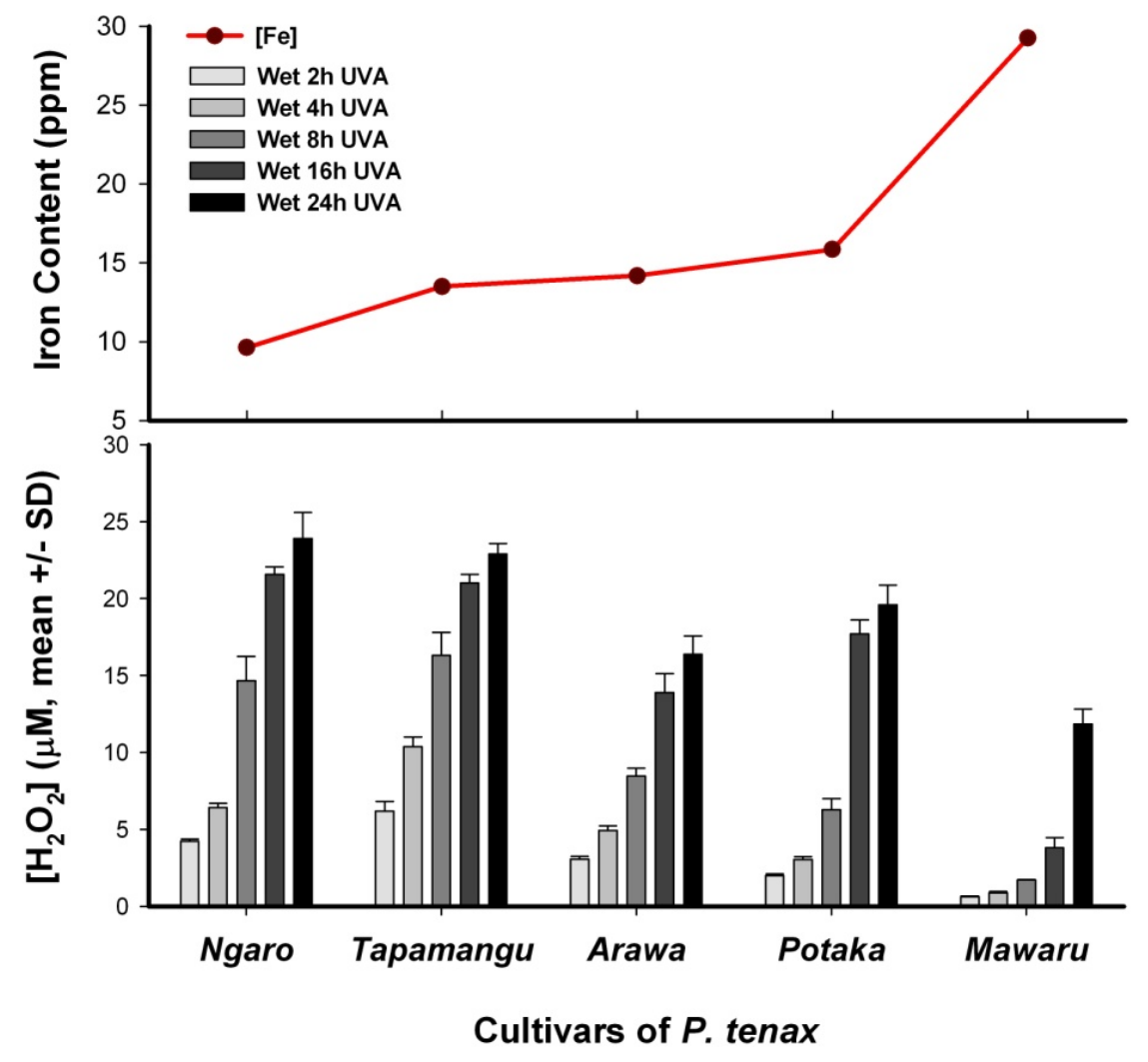

Figure 6.3 Iron levels (dry weight in ppm) of Phormium fibres from five cultivars and the association with their production of $\mathrm{H}_{2} \mathrm{O}_{2}$ after wet UVA-irradiation. $\mathrm{H}_{2} \mathrm{O}_{2}$ is shown as concentration $(\mu \mathrm{M})$ in the water from irradiated fibre over exposure times from 2 to 24 hrs.

As stated in the introductory section of this chapter, the produced $\mathrm{OH}^{*}$ radicals are the most potent oxidising species, which can initiate free-radical reactions leading to the oxidative modifications of lignin model compounds ${ }^{[166]}$. Previous reports have identified $\mathrm{OH}^{*}$ in lignin-rich pulps with an adverse effect on the photostability ${ }^{[185]}$. Moreover, in medical studies, oxidative stress induced by $\mathrm{OH}^{*}$ and other ROS are intimately involved in the development of inflammation ${ }^{[186]}$, apoptosis $^{[187]}$ and brain aging ${ }^{[188]}$. The regulatory role of cellular iron in these 
processes has been widely reviewed ${ }^{[184,189-192]}$, and iron overload was implicated in the pathogenesis of many oxidation and age related diseases ${ }^{[190,193-196]}$.

In this regard, a postulation is that more photooxidation may occur to the fibres with high iron content as a result of the high levels of $\mathrm{OH}^{*}$ radicals, and a further quantification of the produced $\mathrm{OH}^{*}$ radicals could be a focus of future work. However, in the case of Phormium fibres, no obvious correlation is found between iron levels of the five cultivars and their relative rates of yellowing, as indicated by the UVA-induced reductions of reflectance and of fluorescence yields (see Chapter 4, Section 4.3). This likely poor correlation suggests that more mechanisms may also be contributing to the overall photodiscolouration; other factors such as lignin content and free iron availability ${ }^{[197]}$ may be involved.

\subsubsection{Traditional Maori Dyeing: Wood-Ash Mordant}

As discussed in the introduction to this chapter, the hue of Phormium fibres dyed with aqueous extracts of Tanekaha (Phyllocladus trichomanoides) can be greatly altered by rubbing the fibres with the wood ash of Mahoe (Melicytus ramiflorus). Both plant materials used in the traditional dye are tree barks, shown in Figure 6.4.
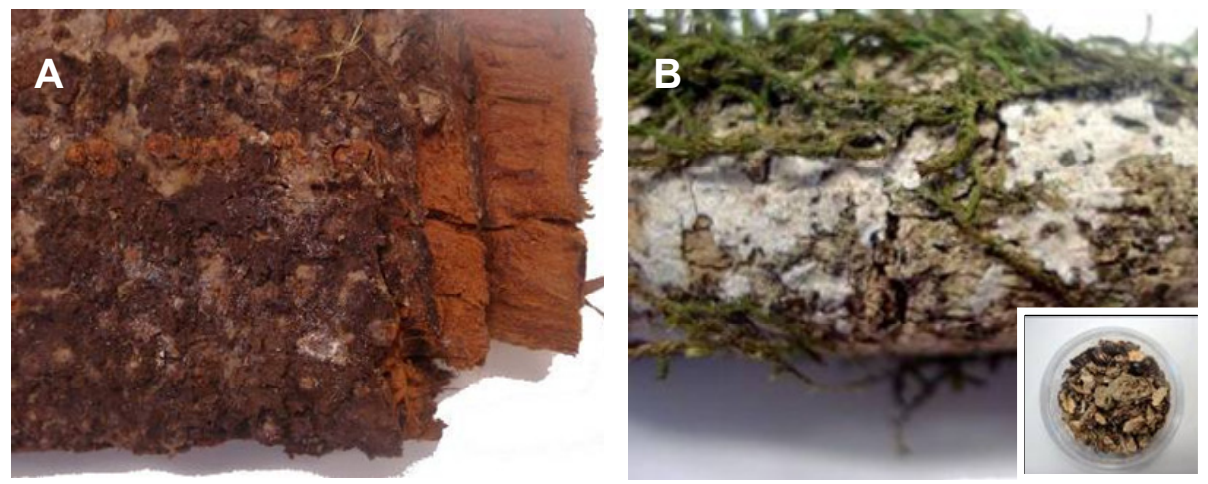

Figure 6.4 Bark samples of Tanekaha (A) and Mahoe (B) with an enlarged view showing the growth of lichen. 
This case study is devoted to (i) establishing the identity of the mordant species in the wood ash of Mahoe, (ii) quantitatively evaluating the elemental difference of wood ash from different sites and (iii) further elucidate the $\mathrm{pH}$ effects on the colour change of dyed fibres, mordanted or not, by UV-Vis spectroscopy.

\section{Optical and Microscopic Observation}

Figure 6.5 shows the SEM micrographs of Tanekaha-dyed Phormium fibres before (A) and after (B) treatment with the wood ash of Mahoe bark. The wood ash treatment caused a colour change of fibres from reddish brown to a dark crimson. In the back-scattered imaging, the particulate matter coating the mordanted fibre is indicative of heavy elements.
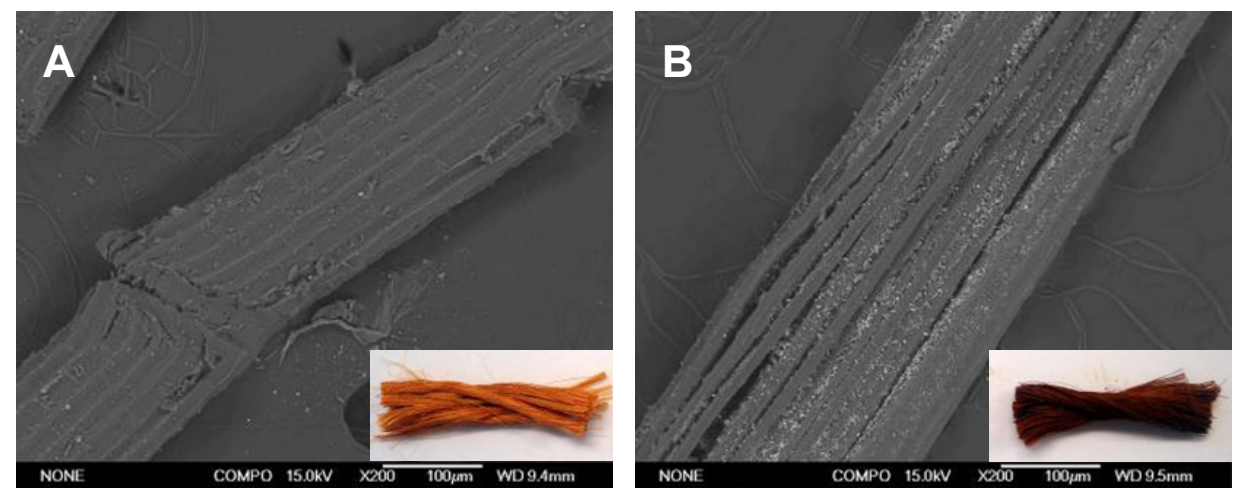

Figure 6.5 SEM back-scattered images of Tanekaha-dyed Phormium fibres without $(A)$ and with $(B)$ treatment of Mahoe ash, magnification: $\times 200$. An optical image of each sample is also present in the bottom right corner.

\section{Elemental Analysis of Wood Ash of Mahoe}

In order to find what elements, particularly heavy or transition metals, are involved as mordants, the elemental compositions of wood ash of Mahoe bark were determined by EDS and XRF analysis. These two X-ray techniques are employed in a complementary way and give the spectra shown in Figure 6.6. 
Their combined elemental information shows that the wood ash had relatively high levels of calcium and iron, with quite low levels of aluminium, potassium and zinc. The most abundant element, calcium, is attributed to the presence of oxides and hydroxides of calcium that commonly are encountered in wood ash and are responsible for the alkalinity of the ash.
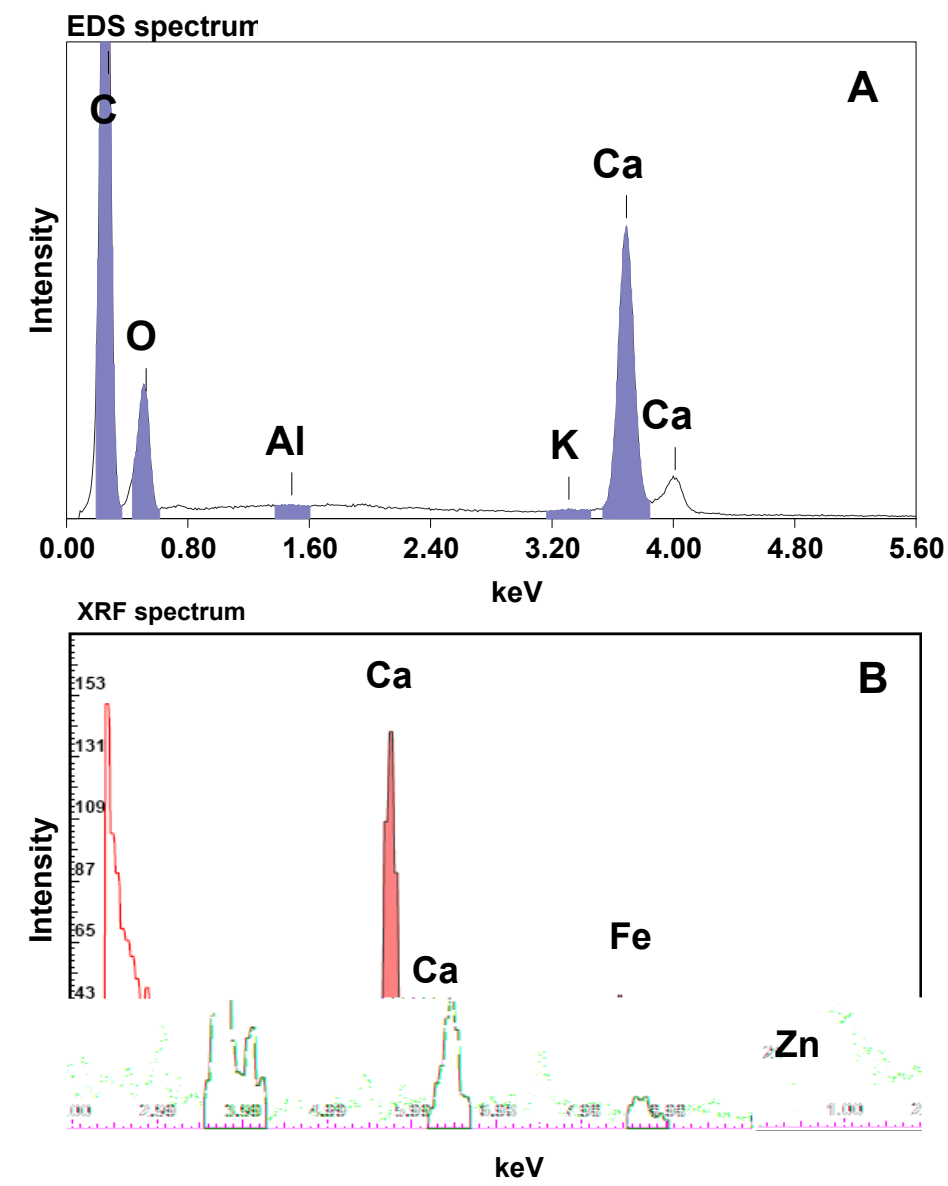

Figure 6.6 X-ray spectra of wood ash of Mahoe bark obtained by EDS (A) and XRF (B) analysis. 
Figure 6.7 shows SEM/EDS micrographs of the cross-section of a mordanted fibre (left column) and a piece of Mahoe bark (right column). The associated EDS mapping indicated that the ash derived from the wood was rich in aluminium, potassium and calcium, while the accumulation of calcium and potassium was associated with the lichen grown on the bark.

This finding indicates that as well as the higher plants, lichens on the bark could be a biological source of mordants although they have seldom been recognised as such. Lichen is a symbiotic dual organism consisting of fungi (mycobiont) and algae (phycobiont) ${ }^{[198]}$. Their ability for absorbing inorganic species from the atmosphere, particularly through the mycobiont part, makes them good biomonitors for airborne metal pollutants ${ }^{[199]}$. Certain species are very tolerant to heavy metals and are regarded as accumulators, such as Xanthoria parietina and Parmelia caperata $^{[200,201]}$. In traditional dyeing in history, lichens have represented a source of natural dyes which are wash-fast on wool and silk and they do not require auxiliary fixing agents (mordants) ${ }^{[162]}$. It is possible that fixing metal ions are present in the dye liquor extracted from the lichen. 

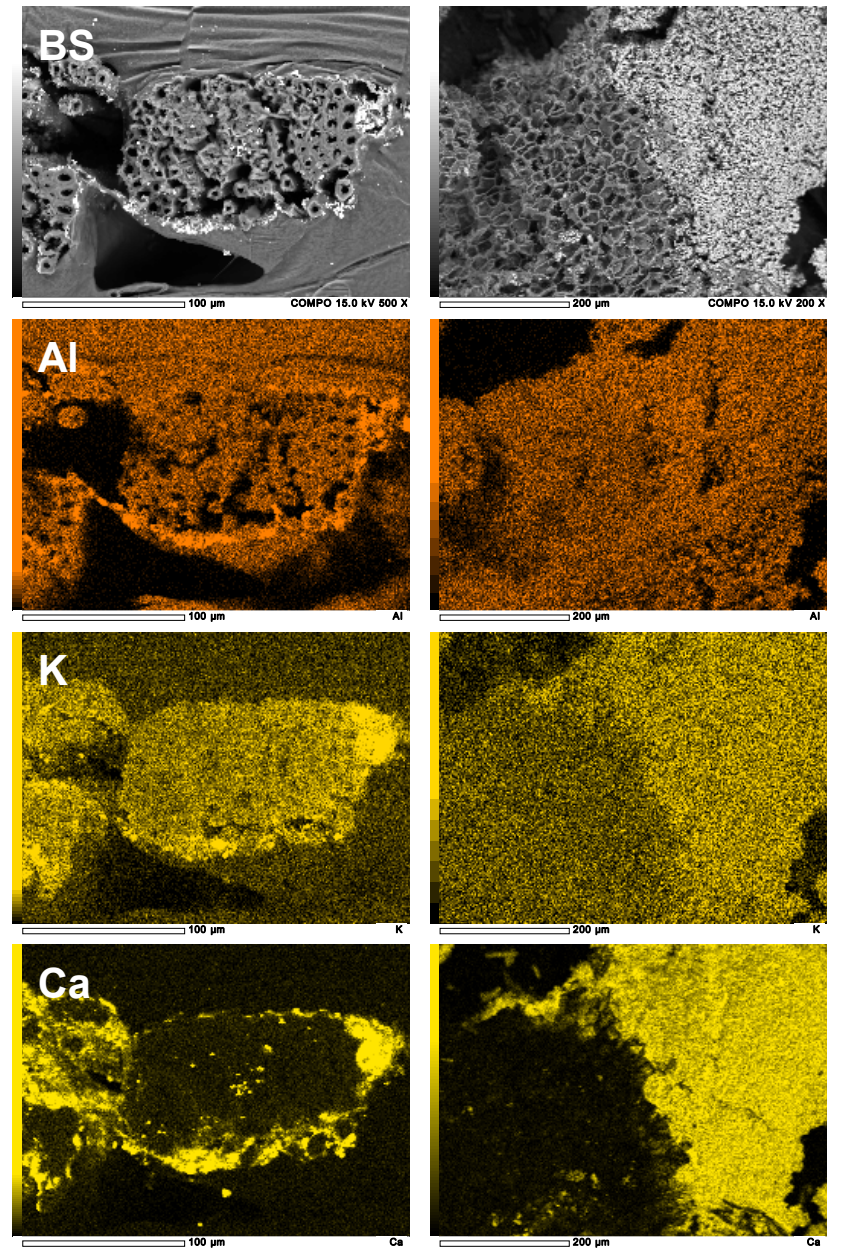

Figure 6.7 Backscatter images (BS) of the cross-section of a Phormium fibre treated with wood ash of Mahoe (left column, magnification: $\times 500$ ) and a piece of Mahoe bark (right column, magnification: $\times 200$ ) with the growth of lichen on the right side, with EDS mapping of $\mathrm{Al}, \mathrm{K}$ and $\mathrm{Ca}$. 


\section{Quantitative Assessment and Geographic Differences}

ICP-MS analysis was employed to quantify the amounts of mordant metals in wood ash and to compare differences among samples from three different sites of New Zealand, viz. Wilton (Wellington), Puriri Bay (Marlborough) and Te Kuiti (Waikato). The results are shown in Table 6.3. The prodominant element detected in all three samples was calcium, in the range of $1.4-3.3 \times 10^{4} \mathrm{ppm}$, which is common in wood ash as it is traditionally the principal source of calcium carbonate. Lower levels of aluminium, iron, copper and zinc were also present, with varying amounts depending on the sampling sites, which may function as mordant metal ions and modify the hue of colour in varying degrees.

Table 6.3 ICP-MS determination of trace elements in ashed samples of Mahoe bark collected from three different regions in New Zealand

\begin{tabular}{|c|c|c|c|c|c|c|c|}
\hline \multirow[t]{2}{*}{ Element } & \multicolumn{6}{|c|}{ Intensity ( $\left.{ }^{*} c p s\right)$} & \multirow{2}{*}{$\begin{array}{l}\text { Conc } \\
\text { (ppm) }\end{array}$} \\
\hline & Sample $(5 \mathrm{~mL})$ & $R S D \%$ & Std (1ppb) & $R S D \%$ & Blank & $R S D \%$ & \\
\hline \multicolumn{8}{|c|}{ Mahoe Ash - Wilton, Wellington $(0.85 \mathrm{mg})$} \\
\hline $\mathrm{Al}$ & 73929.7 & 3.85 & 2681.6 & 7.96 & 122.8 & 10.34 & 170 \\
\hline $\mathrm{Ca}$ & 232915.3 & 1.96 & 109.7 & 9.50 & 9.9 & 35.97 & 13728 \\
\hline $\mathrm{Fe}$ & 24357.4 & 2.27 & 1423.7 & 3.32 & 76.7 & 12.03 & 106 \\
\hline $\mathrm{Cu}$ & 127880.4 & 2.04 & 3474.2 & 3.34 & 270.8 & 8.20 & 234 \\
\hline $\mathrm{Zn}$ & 232535.2 & 1.63 & 2708.0 & 2.52 & 99.1 & 10.80 & 524 \\
\hline \multicolumn{8}{|c|}{ Mahoe Ash - Puriri bay, Marlborough $(1.09 \mathrm{mg})$} \\
\hline $\mathrm{Al}$ & 199959.2 & 3.28 & 2588.7 & 3.54 & 113.5 & 10.20 & 370 \\
\hline $\mathrm{Ca}$ & 616685.7 & 2.36 & 111.0 & 10.82 & 9.2 & 36.93 & 27788 \\
\hline $\mathrm{Fe}$ & 83391.2 & 3.99 & 1549.2 & 3.82 & 67.4 & 13.36 & 258 \\
\hline $\mathrm{Cu}$ & 523241.7 & 1.43 & 3839.6 & 2.64 & 53.2 & 29.38 & 634 \\
\hline $\mathrm{Zn}$ & 70711.5 & 1.40 & 3122.8 & 2.19 & 51.6 & 23.48 & 106 \\
\hline \multicolumn{8}{|c|}{ Mahoe Ash - Te Kuiti, Waikato (1.35 mg) } \\
\hline $\mathrm{Al}$ & 168388.5 & 3.52 & 2623.3 & 3.52 & 120.7 & 9.19 & 249 \\
\hline $\mathrm{Ca}$ & 828352.1 & 1.98 & 106 & 9.79 & 13.7 & 147.32 & 33239 \\
\hline $\mathrm{Fe}$ & 93118.8 & 3.82 & 1452.4 & 3.90 & 74.3 & 14.34 & 250 \\
\hline $\mathrm{Cu}$ & 78397.9 & 1.64 & 3628.6 & 3.00 & 259.8 & 11.45 & 86 \\
\hline $\mathrm{Zn}$ & 40273.1 & 1.25 & 2837 & 2.58 & 95.5 & 11.74 & 54 \\
\hline
\end{tabular}

*cps: count per second 


\section{The pH Effects on Optical Properties of Tanekaha-dyed Fibres}

$\mathrm{pH}$ measurements of two fibre samples dyed with Tanekaha indicated that the mordanted fibres have a $\mathrm{pH}$ of 7.0, whereas the unmordanted fibres are acidic with a $\mathrm{pH}$ of 4.4 . It is apparent that the wood ash of Mahoe mainly serves as a base in the dyeing process.

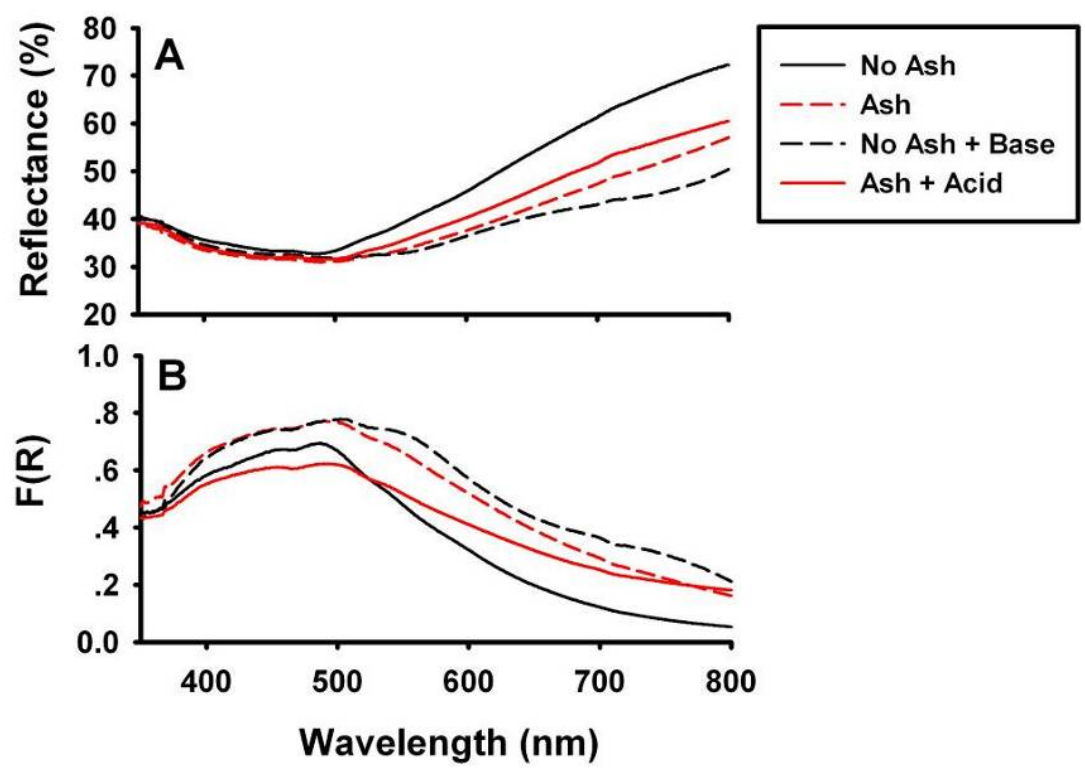

Figure 6.8 Reflectance spectra (A) and Kubelka-Munk transforms (B) of Tanekaha-dyed Phormium fibres with (red) or without (black) rubbing with Mahoe ash, when pH adjusted to 4.4 (solid curve) or 7.0 (dashed curve).

To establish whether the colour change in the Tanekaha dye is a result of the alkalinity of wood ash, the acidity of unmordanted fibres was adjusted with acetate buffer to $\mathrm{pH} 7.0$, while that of mordanted fibres was adjusted to $\mathrm{pH} 4.4$ using hydrochloric acid. Their reflectance spectral changes are displayed in both reflectance values (Figure 6.8A) and in Kubelka-Munk units (Figure 6.8B); the latter is obtained by the transformation, $F(R)=(1-R)^{2} / 2 R$, to make reflectance values (R) appear similar to those of absorbance. It is apparent that there was red shift in the absorption of chromophores from protonated form $(\mathrm{pH} 4.4)$ to deprotonated form ( $\mathrm{pH}$ 7.0) with increasing $\mathrm{pH}$. However, the spectra of fibres of 
identical $\mathrm{pH}$ do not match each other, suggesting there are other factors as a result of treatment with wood ash that influences the hue of dye-fibre chromophores, especially in the wavelength range of $500-800 \mathrm{~nm}$.

The bark of Tanekaha is rich in tannin. Earlier investigations of the cladodes, i.e. dried twigs of the tree reported the isolation and identification of catechin, phylloflavan and phenylpropanoid derivatives of epicatechin (cinchonains) ${ }^{[202,203]}$. As shown in Figure 6.9, these polyphenolic compounds, described as flavans, are members of the tannin group of natural products that are largely responsible for the red-brown colouration of plants. Thus, this work has provided an insight into the structure of products that may also occur in the bark. The characteristic feature of these compounds is the orthodihydroxyphenolic group. Therefore, the wide occurrence of the grouping in the polymeric flavonoids and/or tannins provides these chromophores with the ability to form charge-transfer complexes with mordanting metal ions (see Figure 6.9), which are likely to occur under neutral to basic conditions and can in turn influence the hue of colours.<smiles>[R]C1Cc2c(O)cc(O)cc2OC1c1ccc(O)c(O)c1</smiles><smiles>O=C(CC(O)CCc1ccc(O)c(O)c1)OC#P</smiles>

Phylloflavan analogues of Catechin/Cinchonan<smiles>[R]C1Cc2c(O)cc3c(c2OC1c1ccc(O)c(O)c1)C(c1ccc(O)c(O)c1)CC(=O)O3</smiles>

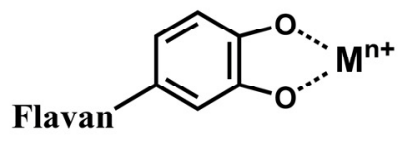

Metal ion complex

Figure 6.9 Structures of polyphenolic products identified in the cladodes of Tanekaha and a proposed modanting mechanism of complexing with metal ions (bottom right corner). 
The fluorescence excitation and emission spectra of the four differently-treated fibre samples were also examined as shown in Figure 6.10. The unmordanted fibres showed a set of fluorophores emitting in the yellow-green range with the maximum at $550 \mathrm{~nm}$, together with a coumarin fluorophore with an emission maximum at $420 \mathrm{~nm}$. When buffered at $\mathrm{pH} 7.0$, the fluorescence intensities of all fluorophores decreased greatly but are still stronger than that of mordanted fibres. The strong fluorescence at $550 \mathrm{~nm}$ could make the fibres appear yellower. In the case of mordanted fibres, acid treatment produced only minor changes in the fluorescence spectral profile but the coumarin fluorophores were completely quenched. Thus, the colour change of Phormium fibres on wood-ash mordanting is more likely due to the production of chromophores absorbing at longer wavelengths rather than by fluorophores emitting in the blue-green spectral region.

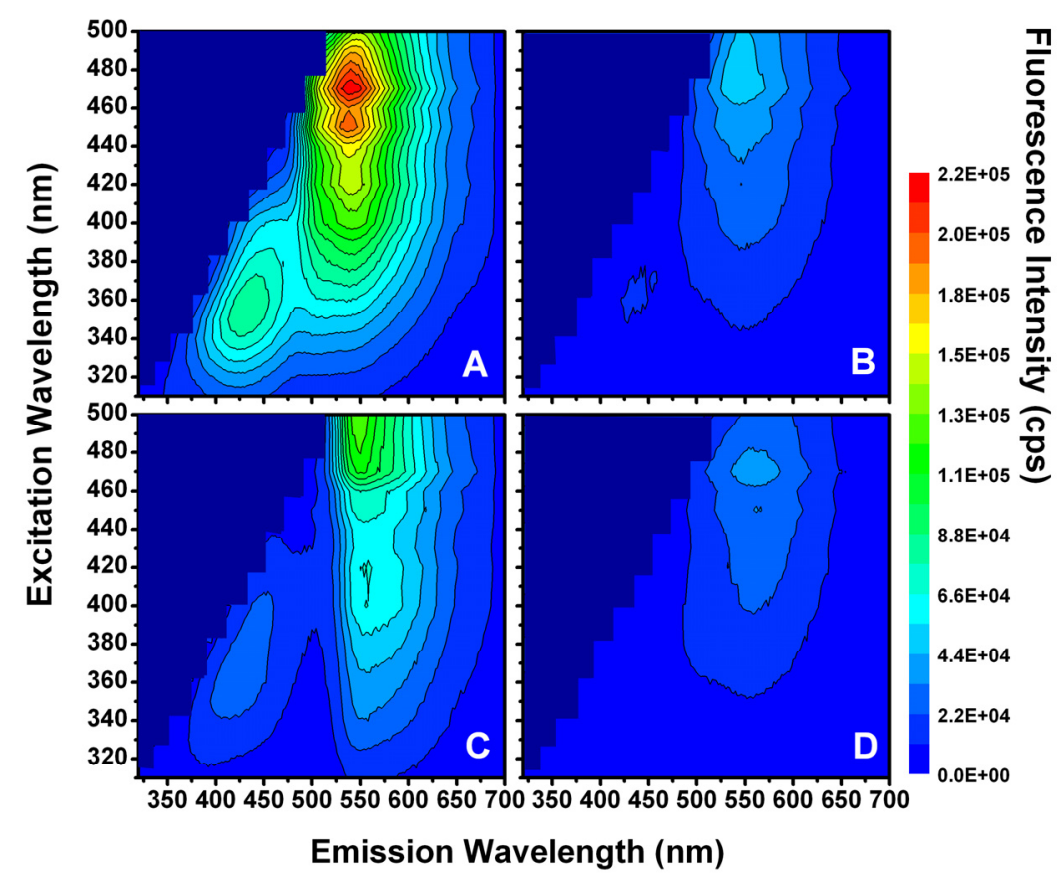

Figure 6.10 3D fluorescence spectra of Tanekaha-dyed $P$. tenax fibres: no mordant $(A)$, mordanted with wood ash of Mahoe $(B)$, no mordant $+\mathrm{pH} 7.0$ buffered $(C)$ and wood ash-mordanted $+\mathrm{pH} 4.4$ buffered (D). 


\subsubsection{Photostability of Alum-Treated Phormium Fibres}

The aim of this study is to evaluate the role of aluminium mordant ion in the fluorescence and photodegradation of Phormium fibres. The optical spectral changes of undyed fibres (cultivar Tapamangu) treated with potash alum at different temperatures, before and after $24 \mathrm{hrs}$ of UVA-irradiation, were examined by reflectance and fluorescence spectroscopies.

\section{Alum-Treatment of Phormium Fibres}

Phormium fibres were treated with alum by soaking the fibres in an aqueous solution of $1 \%$ aluminium potassium sulphate at room temperature overnight or at $90{ }^{\circ} \mathrm{C}$ for $30 \mathrm{~min}$ to $1 \mathrm{hr}$ with gentle agitation. All fibre samples were rinsed in cold DDW and dried in air prior to spectroscopic study and/or UVA-irradiation.

Analysis by SEM/EDS showed particulate matter with high levels of Al, K and S coating the surface of the alum-treated fibre as shown in Figure 6.11. The Phormium fibres bind $\mathrm{Al}^{3+}$ ions readily at room temperature and at $90{ }^{\circ} \mathrm{C}$. 

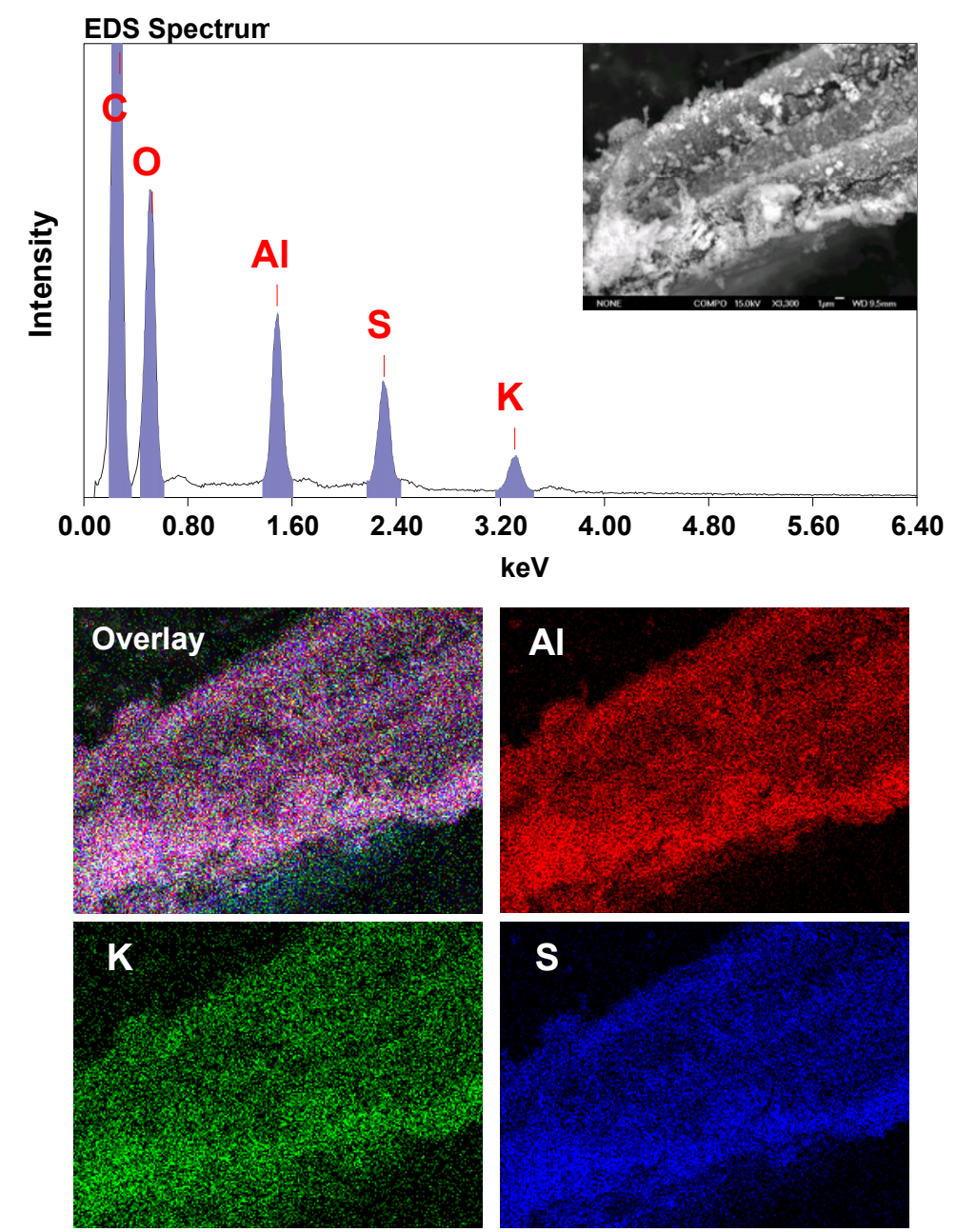

Figure 6.11 The backscatter image (top right corner) of a $90{ }^{\circ} \mathrm{C}$ alum-treated Phormium fibre with associated EDS spectrum and mapping for $\mathrm{Al}, \mathrm{K}$ and $\mathrm{S}$. 


\section{Optical Spectra and UVA-induced Yellowing}

The effects of 24 hrs of UVA-irradiation on the reflectance and 3D-fluorescence spectra of alum-treated and untreated fibres are shown in Figure 6.12 and Figure 6.13 , respectively. The results can be summarised as below:

- In the unirradiated samples (solid curves), it is apparent that yellow chromophores absorbing over the blue region were produced with alum treatment at room temperature (Alum RT); while the treatment at $90{ }^{\circ} \mathrm{C}$ extracted and removed most chromophores from the fibres to produce a bright white fibre (Alum 90). Absorption measurements of aqueous extracts from both the room temperature and $90{ }^{\circ} \mathrm{C}$ treatments (data not shown) have further confirmed this assertion.

- A green fluorophore emitting with a spectral maximum of $500 \mathrm{~nm}$ was formed by treatment with alum at room temperature (Alum RT), which is attributed to an $\mathrm{Al}^{3+}$ complex with coumarin or other polyphenolic residues present in the fibre ${ }^{[204]}$. The fluorophores of Alum 90 fibres is more likely to be produced from the complex anion of $\mathrm{Al}^{3+}$ with anionic functional groups of the fibre.

- Upon irradiation, chromophores absorbing in the blue spectral range (400 $-450 \mathrm{~nm}$ ) were produced, resulting in the yellowing of fibres. Alum 90 fibres displayed a more rapid photoyellowing than the untreated control fibres, whereas Alum RT fibres enhanced the lightfastness of the fibre.

- Instead of fluorescence quenching, as observed with Alum 90 fibres and the control fibres, the emission of the fluorophores in Alum RT fibres was increased after irradiation, which, at least in part, compensated for the photoyellowing and makes Alum RT fibres more photostable than Alum 90 fibres. 

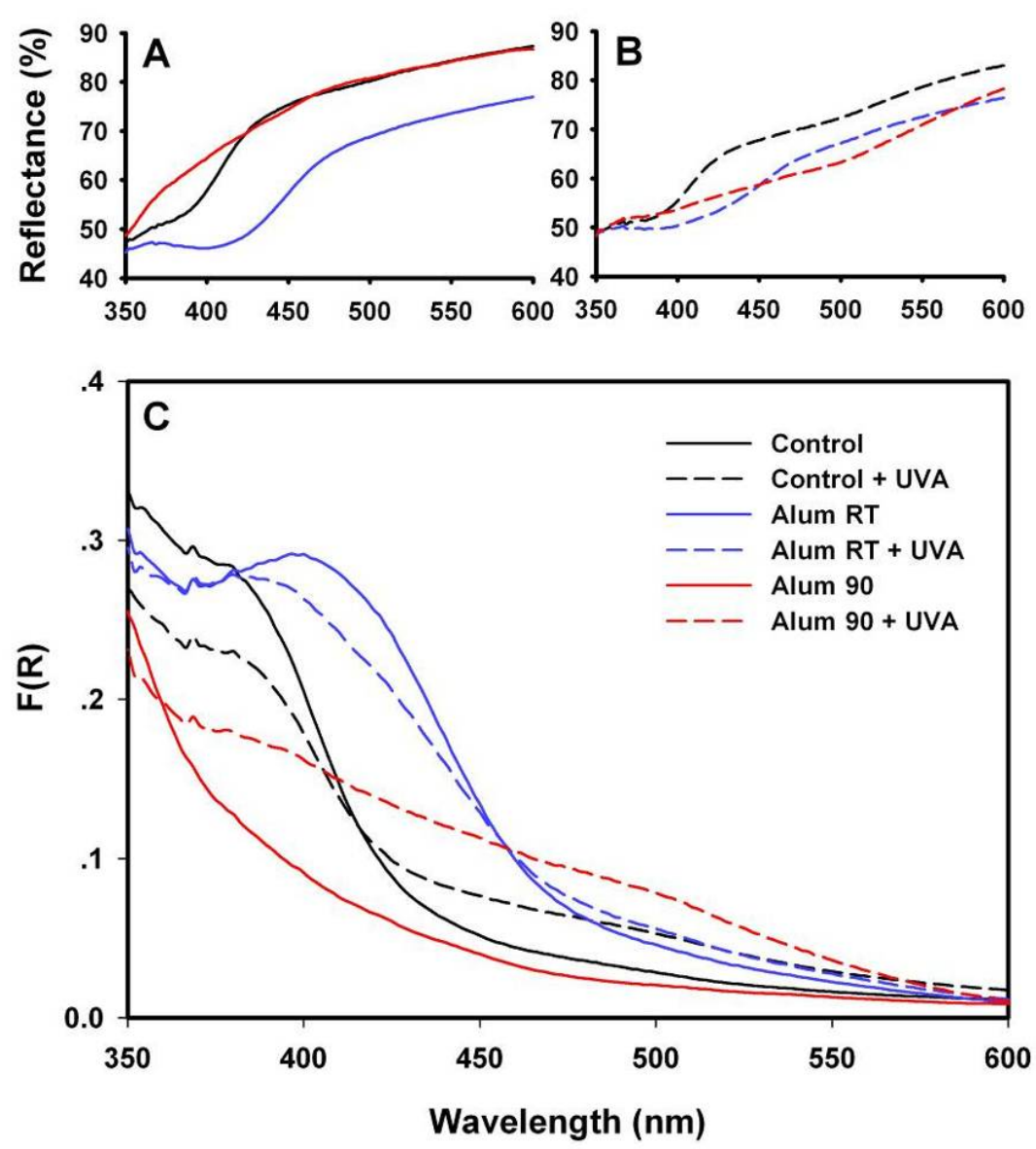

Figure 6.12 Reflectance spectra of differently treated Phormium fibres, no treatment (control), treated at room temperature (Alum RT) or at $90^{\circ} \mathrm{C}$ (Alum 90), before (A) and after (B) 24 hrs of UVA irradiation, and the associated KubelkaMunk transforms (C). 


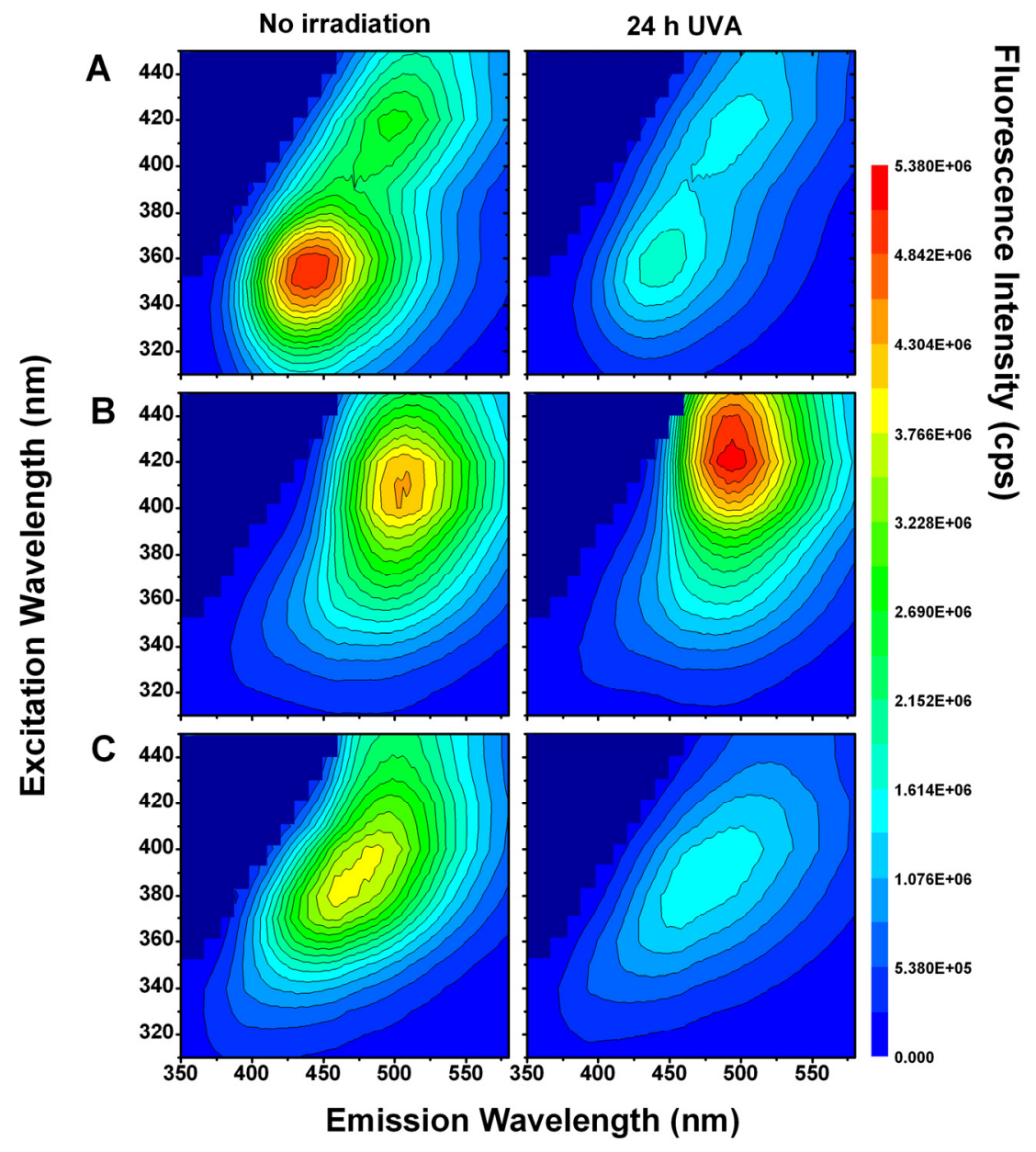

Figure 6.13 3D-fluorescence spectra of differently treated Phormium fibres before (left column) and after (right column) 24 hrs of UVA irradiation: control (A), Alum RT (B) and Alum 90 (C). 


\subsubsection{SEM Investigation of Chinese Handmade Paper}

Characterisation and identification of the plant fibres used in traditional Chinese handmade paper are important for the provenance, authentication, restoration (matching) and conservation of paper-based objects. In this work, the morphological characteristics and elemental compositions of paper samples made of different plant fibres were investigated by SEM-EDS.

\section{Morphological and Elemental Analysis of Three Papers}

Figure 6.14 shows the SEM micrographs of three traditional Chinese handmade papers: Xuan paper, Mulberry paper and Bamboo paper.

- All the papers studied consist of at least two types of fibres; they were made from the mixture of rice straw and other papermaking plants. The morphologies of these fibres have provided valuable information for material identification. For example, the flat fibres with longitudal scales are characteristic of blue sandalwood, while the round, smooth-sheathed fibres are diagnostic for rice straws.

- In the back scattered imaging, localised areas ("bright spots") of heavy elements were observed in Xuan paper and Mulberry paper. The associated EDS analysis indicated that both papers also had high levels of calcium together with a much lesser amount of aluminium. Xuan paper also exhibited a high level of silicon. 


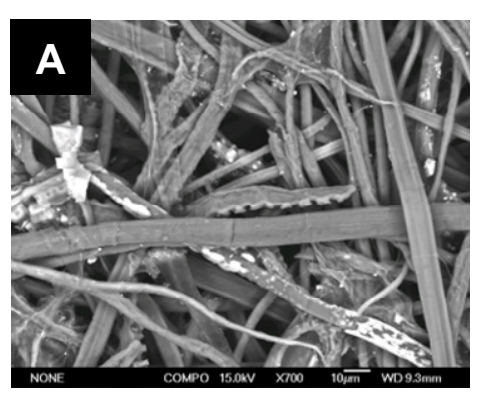

EDS spectrum
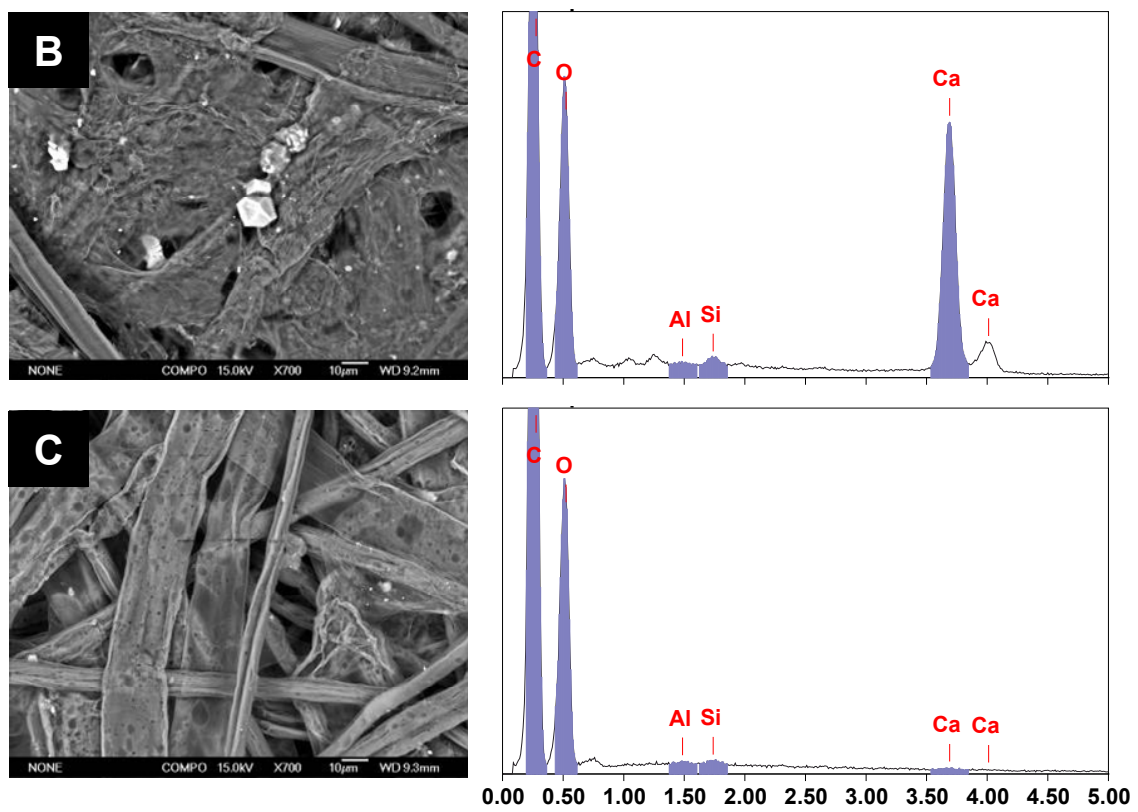

$\mathrm{keV}$

Figure 6.14 SEM backscatter images and EDS spectra of three types of traditional Chinese paper: Xuan paper (A), Mulberry paper (B) and Bamboo paper (C), magnification: $\times 700$ 


\section{EDS Mapping of Xuan Paper and Mulberry Paper}

The EDS mapping of Xuan paper and Mulberry paper for aluminium, silicon and calcium provided further insight into the spatial distribution of these elements, which are shown in Figure 6.15.

- Xuan paper consisted of fibres highly impregnated with aluminium and silicon, which are shown in false colour as "yellow fibres" in the overlaid image of the three elements. Calcium existed as both crystalline particles and bound to the fibres; the distribution of latter also coincided with aluminium and silicon, which appears as "purple fibres" in the false colour overlay image.

- In contrast, Mulberry paper demonstrated an irregular distribution of aluminium and silicon, indicating that these elements are present as finely grained clay minerals. Precipitates of calcium-containing particles were also observed on the surface of a number of fibres, which could be formed in the process of "lime-steaming" used in papermaking (see Chapter 1).

- The difference in the deposition patterns of the two papers could be due to the different raw materials used in their manufacture. Instead of being made of the rice straws that grow in soil as used in Mulberry paper, the rice straw used in Xuan paper is a special species that grows in sandy land. The accumulation of aluminum and silicon is suggested to result from the bio-mineralisation that occurs in this rice straw. 

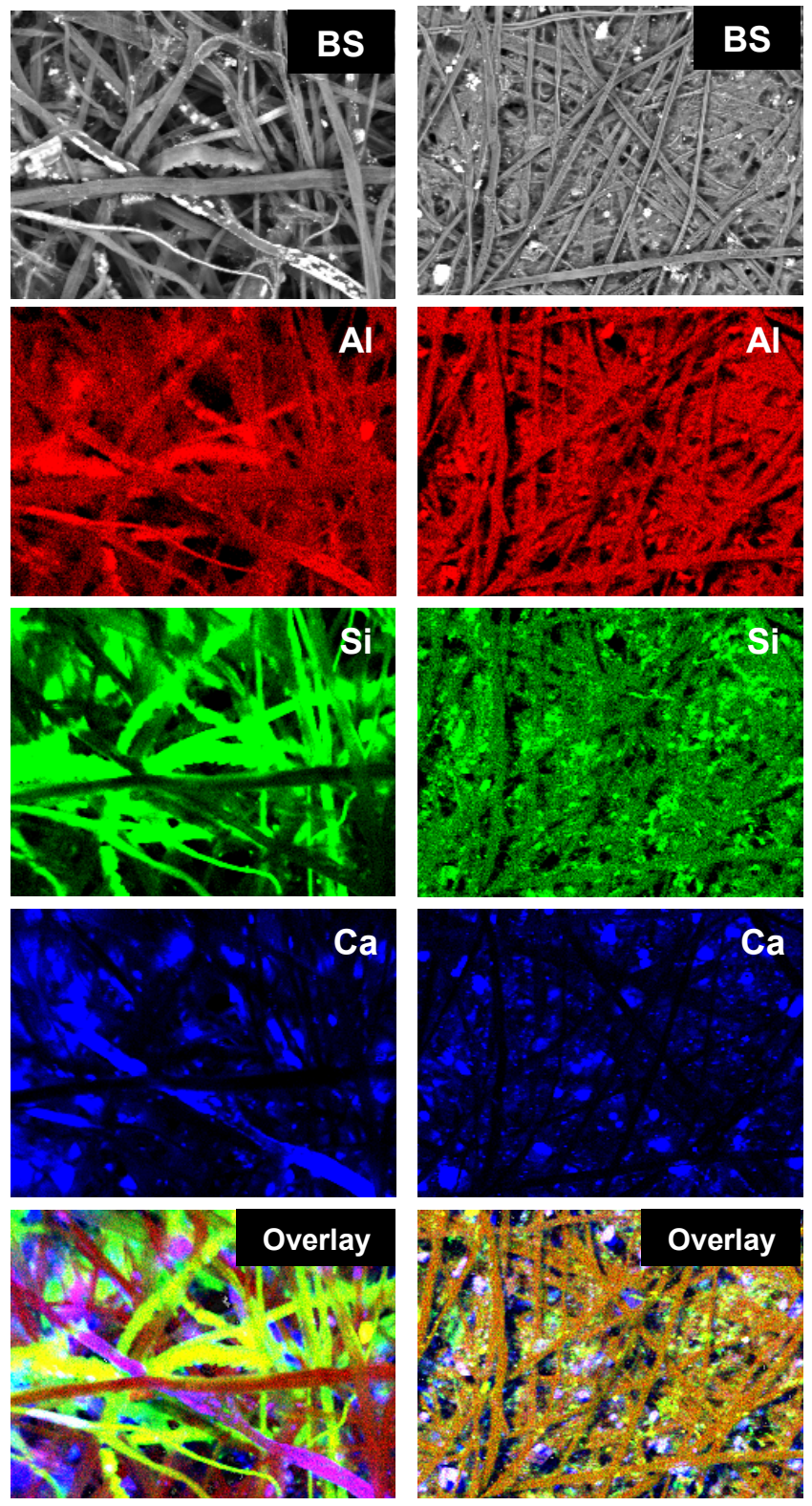

Figure 6.15 SEM Backscatter images (BS, magnification: $\times 700$ ) and false colour EDS images of Xuan paper and Mulberry paper, mapping for Al, Si and Ca. 


\section{EDS Spot Analysis of Xuan Paper}

EDS spot analysis over a diameter of $\sim 5 \mathrm{~nm}$ at selected areas of the sample was performed on Xuan paper. As shown in Figure 6.16, elongated phytoliths of calcium and silicon were observed, which are characteristic of the rice straw grown in sandy land ${ }^{[44]}$. Micron-sized particles containing high levels of calcium were widespread in the paper fibres, which could be derived from the limesteaming process used in the traditional pulping (see Chapter 1).
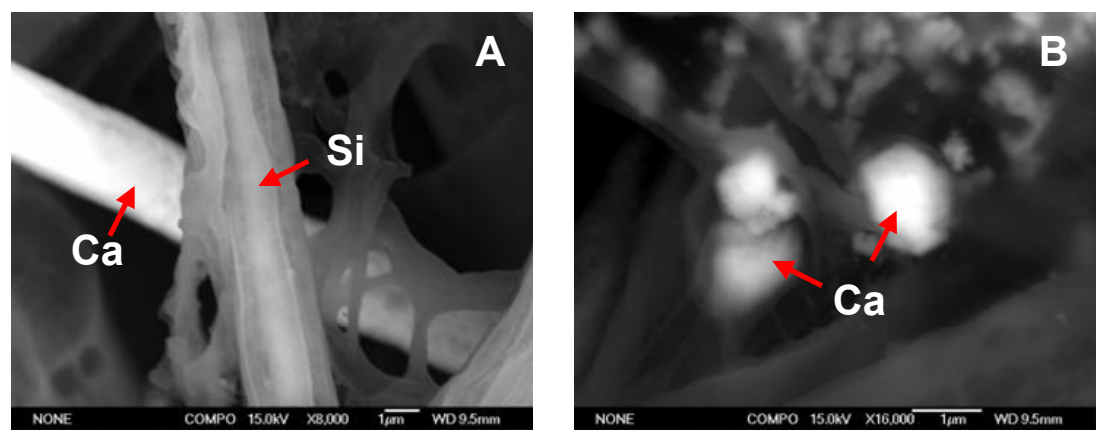

Figure 6.16 EDS spot analysis of Xuan paper, BS image, magnification: $\times 8000$ for the image on the left and $\times 16,000$ for the image on the right

The acidities of three Xuan papers were measured before and after accelerated ageing at $70{ }^{\circ} \mathrm{C}$ in water for 4 days (Table 6.4). The paper samples with calcium precipitates exhibited little change in $\mathrm{pH}$ upon thermal aging, which indicates the efficiency of the micron-sized calcium-containing particles in neutralising any acid produced by hydrolysis. 
Table $6.4 \mathrm{pH}$ values of Xuan paper before and after 4 days of accelerated

\begin{tabular}{c|ccc}
\multicolumn{3}{c}{ aging $\left(100 \% \mathrm{RH}, 70{ }^{\circ} \mathrm{C}\right)$} \\
\hline \multirow{2}{*}{ Xuan paper } & \multirow{2}{*}{${ }^{*}$ Ca ppt } & \multicolumn{2}{c}{ Thermal Aging } \\
\cline { 2 - 4 } & & 0 days & 4 days \\
\hline X1 & +++ & 8.1 & 8.2 \\
X2 & ++ & 8.0 & 8.1 \\
X3 & + & 6.6 & 6.1 \\
\hline
\end{tabular}

*Percentage calcium precipitates in the sampling area: +, $10 \%$; ++, $40 \%$; +++: $60 \%$

\section{Pingao Grass (Desmoschoenus spiralis)}

Pingao is golden sand sedge endemic to New Zealand. Pingao is valued by Maori weavers for its bright orange leaves that are widely used for plaiting and as ornamental elements in tukutuku panels.
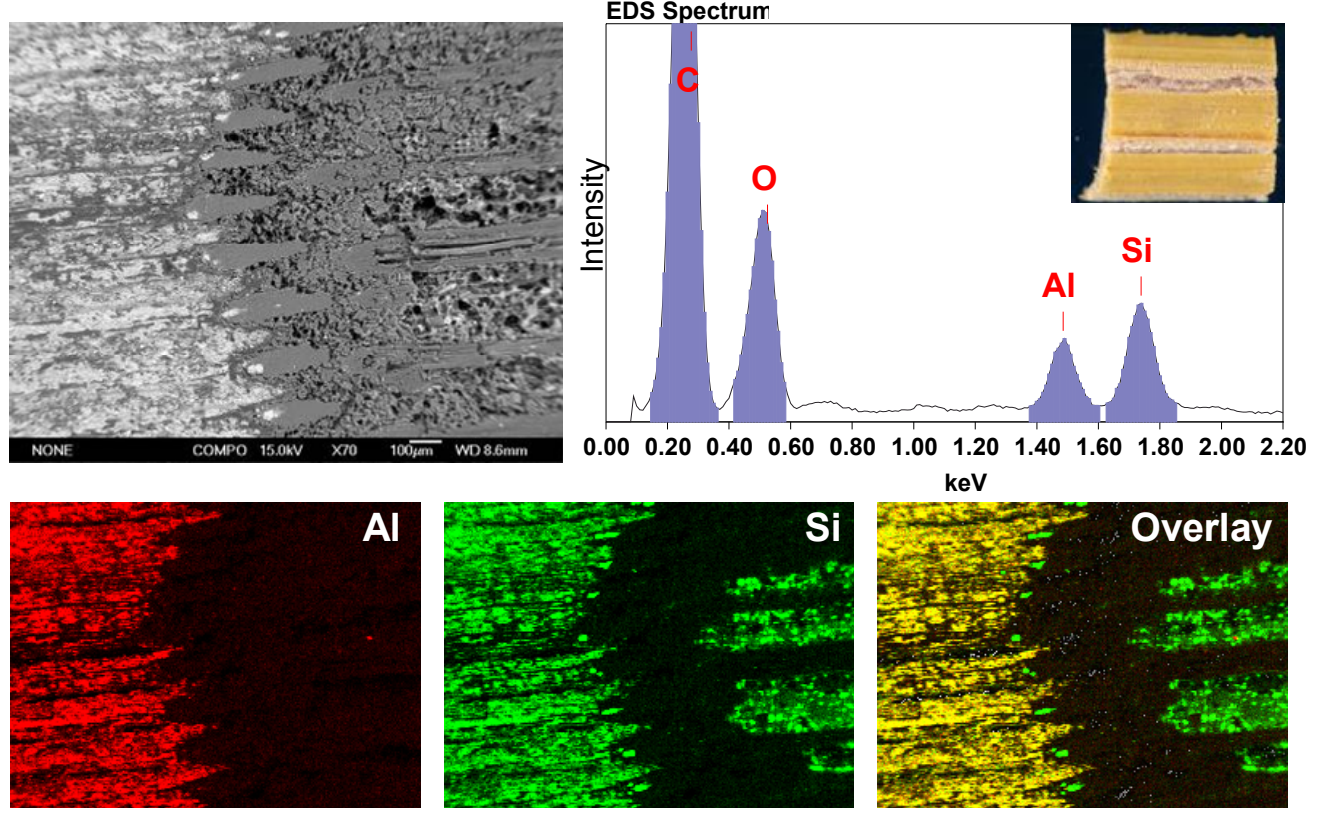

Figure 6.17 Back-scattered image of a Pingao grass with the associated EDS spectrum and false-colour mapping for $\mathrm{Al}$ and $\mathrm{Si}$; magnification: $\times 70$. An optical image of a portion of the grass is also shown in the top right corner.

Both the surface and the cross-section of Pingao leaf were investigated by SEMEDS analysis. As shown in the BS image of Figure 6.17, the grass is found to 
have a coverage of heavier than carbon elements in the surface layer, and the associated EDS mapping indicate that this material had high levels of both aluminium and silicon. In the overlay view, both elements were seen on the surface of grass, the orange-yellow colour of which could be attributed to the complexes formed between the flavonoids and aluminium ions ${ }^{[204]}$ present in the outer layer. However, silicon was only element shown in the middle of the crosssection of grass, suggesting the metal uptake of silicon from sand is preferentially accumulated in the inner vascular tissue of the plant.

\subsection{Summary of the Chapter}

The elemental information of heritage fibrous materials is of fundamental significance to the understanding of chemistry of fibre deterioration and developing appropriate conservation strategies. Four facets of the involvement of metal ions in Phormium fibres, traditional Chinese handmade papers and Pingao grass were addressed in this chapter via four case studies:

\section{Case I: Fenton Chemistry}

The iron contents of Phormium fibres of five cultivars were quantitatively determined by atomic absorption spectroscopy. Mawaru, the cultivar with the lowest yield of $\mathrm{H}_{2} \mathrm{O}_{2}$ upon UVA-irradiation was found to have the highest content of iron, which supports the hypothesis that $\mathrm{H}_{2} \mathrm{O}_{2}$ is consumed to produce hydroxyl radicals via the iron-catalysed Fenton reactions.

\section{Case II: Mordant of Natural Dyes}

The second study was based on the observation that when Phormium fibres dyed reddish brown by the bark solution of Tanekaha (Phyllocladus trichomanoides) are rubbed in the warm ashes of Mahoe (Melicytus ramiflorus) bark, the colour turns a dark crimson colour. Calcium (oxide/hydroxide) was found as the principal 
element in the wood ash, which accounts for the alkalinity of mordanted fibres. Trace amounts of aluminium, iron, copper and zinc were also present and it is suggested these metal ions produce subtle changes to the hue of the dyed fibres. ICP-MS analysis found variations in metal ion compositions of the wood from different geographic regions. Spectroscopic studies indicated that the colour change of Phormium fibres caused by wood ash mordanting/treatment is likely to be due to the production of non-fluorescing chromophores of the Tanekaha dye absorbing at longer wavelengths

\section{Case III: Alum}

Undyed Phormium fibres were treated with potash alum and exposed to UVAradiation to evaluate the role of $\mathrm{Al}^{3+}$ ions in the photodegradation by reflectance and fluorescence spectroscopies. Treatment with alum at room temperature demonstrated enhanced lightfastness on irradiation with the formation of yellow chromphores as well as a green fluorophore. By contrast, fibres treated with alum at $90{ }^{\circ} \mathrm{C}$ were found to be more subject to photoyellowing.

\section{Case IV: Phytolith and Calcium Precipitates}

The morphological characteristics and elemental compositions of three traditional Chinese handmade papers were investigated by SEM/EDS. Phytoliths of calcium and silicon characteristic of the rice straw grown in sandy land were observed in Xuan paper. Micron-sized calcium precipitates were shown to give protection against the acidification of paper during thermal ageing. A comparative investigation of pingao grass revealed that silicon may exist as a biomineral in the inner vascular system of the leaf in the form of a phytolith. 


\section{Chapter 7}

\section{Closing Discussion and Conclusions}

"Real knowledge is to know the extent of one's ignorance." - Chinese philosopher Confucius (551 - 479 BC)

\subsection{Concluding Summary}

Coumarins are a group of compounds that are found in many plants and many of them have antimicrobial activity. Naturally-occurring coumarins were previously detected in Phormium fibres and are suggested to contribute to the fluorescence and photodegradation of the fibre ${ }^{[1]}$.

This thesis has extended the study on Phormium fibres to both bioactivity and photostability. The primary aims are (i) to assess the antifungal activity of Phormium fibres of different weaving cultivars and identify coumarins and other antifungal agents; (ii) to evaluate the role of coumarins in the photodegradation of fibres during UVA-irradiation; (iii) to further elucidate the mechanism behind the photochemical behaviours; (iv) to investigate the effects of mordant metal ions on the spectral reflectance and fluorescence properties of dyed and/or undyed fibres.

A parallel study was also carried out on Chinese handmade papers looking at their UVA-induced optical spectroscopic changes in relationship to the manufacturing techniques used. Some of the main findings are summarised as follows. 


\subsubsection{Summary of Main Findings}

\section{Phormium Fibres}

A screening of antifungal activity was performed on Phormium fibres of 14 genetically maintained cultivars, including an "ImageJ" evaluation of fungalinduced colour change of solid fibres inoculated with Penicillium grabrum and Aspergillus wentii, as well as a standard colorimetric assay of the aqueous extracts of fibres in vitro ${ }^{[65]}$. The tested cultivars of $P$. tenax showed a range of antifungal activities in both assays. The antifungal activities of four authentic coumarins were also assessed. Coumarin and 7-hydroxycoumarin showed some activity, with the minimum inhibitory concentrations (MICs) in the range of $100-400 \mu \mathrm{g} \cdot \mathrm{mL}^{-1}$ against Penicillium and Aspergillus.

Some compounds with antifungal activities were identified in the aqueous extracts of Phormium fibres by ESI-MS/MS. These included furfural, coumarin and four 7-hydroxylated derivatives.

Phormium fibres exhibit strong fluorescence in the violet-blue spectral region that is consistent with the emission originating from 7-hydroxycoumarins. The photoyellowing of Phormium fibres caused by UVA irradiation is likely to result, in part, from the coumarin-photosensitised formation of photoproducts absorbing and emitting at longer wavelengths as well as a substantial loss of the blue fluorescence as a consequence of the photodegradation of the 7hydroxycoumarins present in the fibres. A more rapid yellowing of fibres was observed when they were irradiated in the presence of water.

Two of the hydroxycoumarins, identified in Phormium fibres, 6, 7dihydroxycoumarin and 7-hydroxy-6-methoxycoumarin were subjected to UVA photolysis in aqueous solution and two primary photoproducts have been tentatively identified using ESI-MS/MS; a photodimer containing a linking cyclobutane ring and a monomeric photooxidation product. Neither of these 
products has been previously reported.

It is concluded that at least some of photoproducts are produced from the reaction of coumarin and other molecules in the fibres with reactive oxygen species, including hydrogen peroxide and superoxide radicals, generated via an electron transfer mechanism with coumarins in their excited singlet states.

From these results, some deductions can be made regarding the intercultivar differences observed in the Phormium fibres studied:

- The aqueous extract of the Phormium cultivar Mawaru showed that the highest antifungal activity in the in vitro aqueous assays (Chapter 2) also possessed the greatest variety of known antifungal agents identified by ESI-MS/MS (Chapter 3), which could have implicated a synergistic effect.

- Of the solid fibres selected for study in Chapter 4, there is a correlation between their relative photosensitivities and resistance to fungal infection: the three most highly fungal-resistant cultivars, Ngaro, Tapamangu and Arawa, were apparently more fluorescing and more susceptible to UVAinduced photoyellowing than the other two cultivars, Mawaru and Potaka, that exhibited moderate to weak antifungal activity in the solid fibre assay.

- In Chapter 6, the relative iron levels of the five cultivars, Mawaru > Potaka $>$ Arawa $>$ Tapamangu $>$ Ngaro, was inversely related to their photogeneration of $\mathrm{H}_{2} \mathrm{O}_{2}$ (Chapter 4). The Fenton reaction involving ferrous ions was suggested to be responsible for the trend.

Thus, this study has provided useful information for fibre selection in creating Phormium heritage objects or textile fibre blends with desirable properties such as intrinsic resistance to fungal infection and/or good photostability. 


\section{Chinese Handmade Paper}

In Chapter 5, a study of similar design to that used for the study of Phormium fibres was carried on three major types of Chinese handmade paper, i.e. Xuan paper, Mulberry paper and Bamboo paper, looking at their spectroscopic and photochemical properties.

The 3D-fluorescence spectra of unirradiated Xuan paper and Mulberry paper were similar to that observed for Phormium fibres, while the Bamboo paper was characteristic of multiple fluorophores. Exposure of the papers to UVA radiation resulted in decreases in the fluorescence intensities and reflectance values. The determinations of photoinduced hydrogen peroxide and superoxide radical production suggested an electron transfer mechanism was involved in the photodegradation of the papers studied.

The effect of different manufacturing techniques on the relative photostabilities of Chinese handmade paper follows the trend: traditional pulp + natural bleach $>$ traditional pulp + chemical bleach $>$ traditional pulp + unbleached $>$ chemical pulp/bleached. For papers that were stated "to be traditionally produced", the three Xuan papers in particular, differences in their photostabilities could be due to the different amounts of lignin and trace metal ions in the raw materials.

In the scanning electron microscopic examination and elemental analysis of paper samples described in Chapter 6, Xuan papers were found to have phytoliths of calcium and silicon characteristic of the use of a rice straw grown in sandy soil in the pulp. Also, micron-sized calcium precipitates were observed in all traditionally manufactured papers in varying amounts, which could have derived from the lime-steaming process used in the traditional papermaking. These calciumcontaining particles were shown to be effective in the deacidification of paper during thermal ageing.

Therefore, this work has provided some insights into the selection of appropriate paper for the conservation of heritage objects made of Chinese handmade papers. 


\subsection{Limits of Current Study and Future Work}

Some questions and limits also emerged in the current assays of the thesis, which provide a basis for the design of further and/or new studies.

\section{Antifungal Assay}

In the mycological screening described in Chapter 2, no correlation was found between the antifungal activities obtained from aqueous extracts of Phormium fibres with those from the solid fibre assays. There are other factors likely to contribute to the overall antifungal activities of Phormium fibres and variables, such as nutrient status and/or the presence of other antifungal agents besides coumarin could have important effects.

To establish the role of naturally-occurring coumarins in the antifungal activity of Phormium fibres, the coumarin species need to be isolated from the fibres, and examined as single compounds or in combinations to establish whether there are synergistic effects. Furthermore, molecular biological experiments such as in vivo observation and genetic profiling are required to elucidate the antifungal mechanism. However, these biological studies are beyond the scope of this thesis.

\section{Photoproduct Analysis}

While the molecular formulae of the photoproducts of the hydroxycoumarins described in Chapter 4 have been determined by ESI-MS/MS, this technique does not allow assignments of the structures. To establish these, larger scale photolysis is required to have sufficient material for NMR examination and analysis.

It will also be important in the future work to carry out experiments with separate reactive oxygen species such as hydrogen peroxide to further elucidate the underlying mechanism of sensitised photooxidation. 


\section{Photodegradation of Chinese Handmade Paper}

A feature of the Chinese handmade papers studied in Chapter 5 is that irrespective of the plant materials employed in their manufacture, papers produced from traditional pulps are more subject to photoyellowing when irradiated in the dry state than is the case when irradiated wet. This finding is the opposite of what has been previously reported for UV-irradiated Phormium fibres and other lignocellulosic fibres. Assessments of the associated photogeneration of hydrogen peroxide and superoxide radicals showed that papers manufactured from chemical pulps generally produce more ROS than those made from traditional pulps.

To enable a better understanding of this distinctive photochemical behaviour, the fluorescing species present in the traditionally-and chemically-pulped papers need to be identified, and longer irradiations of papers are required to track the modification of these primary fluorophores as well as the formation of later-stage photoproducts which may contribute significantly to changes of colour and fluorescence properties. 


\section{Appendix A}

\section{Mycological Protocols}

\section{Subculture and Preservation}

1) Inoculate the stock strains on a petri dish/plate of Potato dextrose agar (PDA) and incubate at $25^{\circ} \mathrm{C}$ for 14 days for conidia production;

2) Seal the culture (labelled " $\mathrm{S}_{1}$ " for subculture 1) with Parafilm, store at $4{ }^{\circ} \mathrm{C}$;

3) Subcultures are obtained by inoculating $S_{1}$ on petri dishes of carrot root extract agar medium every 2 to 3 weeks, and are labeled as $S_{2}, S_{3}$, etc.

\section{Slant preservation}

1) Inoculate $S_{1}$ on a PDA slant in a glass bottle.

2) Seal the bottle with Parafilm and store at $4{ }^{\circ} \mathrm{C}$ (for up to 3 to 6 months).

\section{Filter paper preservation}

1) Place a strip of filter paper $(0.5 \times 1.2 \mathrm{~cm})$ in an ampoule cupped with cotton wool $(0.6 \times 8 \mathrm{~cm}), 121^{\circ} \mathrm{C}$ autoclave for $30 \mathrm{~min}$;

2) Immerse the strip in a dense conidial suspension, and insert it back to the ampoule;

3) Dry the ampoule in a desiccator and seal with parafilm, the strain-contained ampoule can be stored at $-20^{\circ} \mathrm{C}$ in the dark for up to 2 years. 


\section{Medium Preparation}

\section{Potato dextrose agar growth medium (PDA, Roth)}

1) Weigh $7.8 \mathrm{~g}$ of PDA powder and dissolve in $200 \mathrm{~mL}$ of double distilled water (DDW) to make a final concentration of $39 \mathrm{~g} \cdot \mathrm{L}^{-1}$;

2) $121^{\circ} \mathrm{C}$ autoclave for $15 \mathrm{~min}$, pour into 10 to 12 petri dishes.

\section{Carrot root extract agar growth medium}

1) Cut a carrot root (10 cm long) and $50 \mathrm{~g}$ of potatoes into fine pieces;

2) Immerse these pieces in $500 \mathrm{~mL}$ of DDW, and heat to boiling for $20 \mathrm{~min}$;

3) Filter the aqueous extract into two $200 \mathrm{~mL}$ volumetric flasks using No.1 Whatman filter paper, and store at $4{ }^{\circ} \mathrm{C}$ for 2 to 4 weeks;

4) Add $3 \mathrm{~g}$ of agar to $200 \mathrm{~mL}$ of the extract $\left(15 \mathrm{~g} \cdot \mathrm{L}^{-1}\right), 121^{\circ} \mathrm{C}$ autoclave for 15 min, and pour into 10 to 12 petri dishes.

\section{RPMI-1640 broth medium (autoclavable, Sigma-Aldrich)}

1) Weigh $5.2 \mathrm{~g}$ of RPMI-1640 powder; dissolve in $300 \mathrm{~mL}$ of MOPS (4morpholinepropanesulfonic acid, 1.165 M, Sigma-Aldrich);

2) Adjust to $\mathrm{pH} 7.0$ with $\mathrm{NaOH}(0.1 \mathrm{M})$, then add $\mathrm{DDW}$ to make a final volume of $475 \mathrm{~mL}$;

3) Divide the solution into five portions $(95 \mathrm{~mL})$ in five $100 \mathrm{~mL}$ volumetric flasks, autoclave at $121{ }^{\circ} \mathrm{C}$ for $15 \mathrm{~min}$, cool down and store at $4{ }^{\circ} \mathrm{C}$ until use. 


\section{Conidial Suspension and Quantitaion}

1) Cover 7-day-mature colonies with $2 \mathrm{~mL}$ of sterile $0.9 \%$ saline (add 1 drop of Tween 20 for Asperguillus) to harvest approximate $1.5 \mathrm{~mL}$ of the mixture of conidia and mycelia fragments into a sterile $1.5 \mathrm{~mL}$ eppendorf tube;

2) Allow the heavy particles to settle under room temperature, then transfer the supernatant to a clean eppendorf tube and diluted it with $0.9 \%$ saline to obtain a set of lower concentrations. Normally, the dilutions are 1:50 for Penicillium and 1:5 for Aspergillus;

3) Adjust the transmittance at $530 \mathrm{~nm}$ of diluted suspensions to lie within the range of $80-82 \%$ for Aspergillus, $88-90 \%$ for Penicillium using a UV/Vis spectrophotometer;

4) Plate $10 \mu \mathrm{L}$ of a 1:100 dilution of the adjusted conidial suspension on PDA and count the visible colonies after 48 hrs of incubation to ensure the concentration of viable conidia in the range of $0.4 \times 10^{4}-5 \times 10^{4} \mathrm{CFU} \cdot \mathrm{mL}^{-1}$;

5) Conidial suspensions are stored at $4{ }^{\circ} \mathrm{C}$ for single use within a month. 


\section{Fungal Susceptibility Test on Fibres}

1) Weigh $0.1 \mathrm{~g}$ of finely-chopped fibres and press into a $13 \mathrm{~mm}$ diameter compact disc using an hydraulic press;

2) Wrap each disc in tin foil and autoclave at $121^{\circ} \mathrm{C}, 15 \mathrm{~min}$;

3) Load discs into 24-well plate and inoculate each disc with $400 \mu \mathrm{L}$ of conidial suspension (500 $\mathrm{CFU} \cdot \mathrm{mL}^{-1}$ ), controls receive $400 \mu \mathrm{L}$ of DDW only. Each treatment is repeated in triplicate (Fig. A1);

4) Incubate the plate with discs at $25^{\circ} \mathrm{C}$ statically, keep the plate in a doublebottomed plastic box containing DDW to maintain the moisture;

5) Observe fungal growth on the fibre discs daily under optical microscope and take a digital photographic image after 5 to 7 days of incubation.

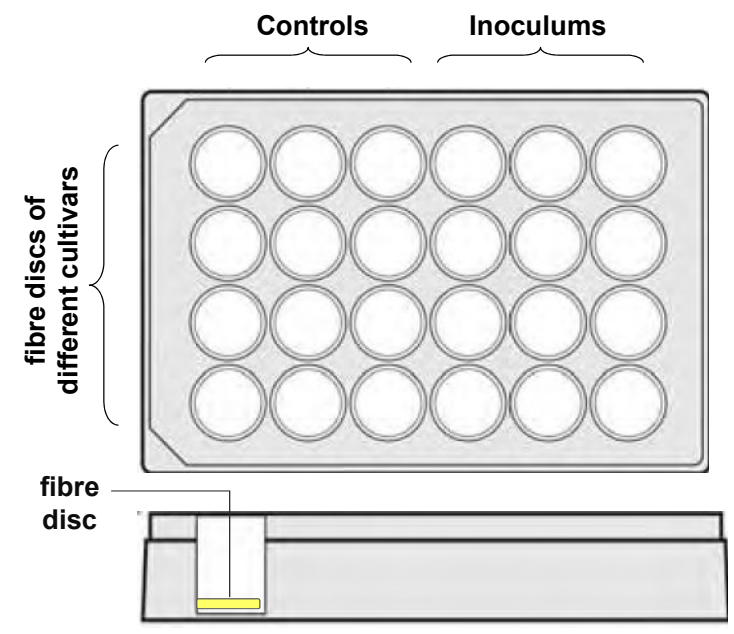

Figure A1. Loading of fibre discs into 24-well plate and the arrangement of controls and inoculums 


\section{Antifungal Susceptibility Test on Solutions}

\section{Inoculum Preparation}

1) Pre-warm $95 \mathrm{~mL}$ of RPMI-1640 medium to $56{ }^{\circ} \mathrm{C}$, add $30 \mathrm{mg}$ of L-glutamine (Sigma-Aldrich) in $4 \mathrm{~mL}$ of DDW (filter-sterilised);

2) Add $1 \mathrm{~mL}$ of CFU-quantitated conidial suspension and well mixed;

3) Plate $10 \mu \mathrm{L}$ of the prepared inoculum on a PDA plate as a visual indicator.

\section{Dilution of Chemicals}

A series of 2-fold diluted antifungal agents are prepared as described below:

Table A1. Scheme for preparing dilution series of water-insoluble antifungal agents to be used in broth dilution susceptibility tests

\begin{tabular}{|c|c|c|c|c|c|c|c|}
\hline Step & $\begin{array}{l}\text { Concentration } \\
\left(\mathbf{m g} \cdot \mathrm{mL}^{-1}\right)\end{array}$ & Source & $\begin{array}{c}\text { Volume } \\
(\mu L)\end{array}$ & $\begin{array}{l}\text { + Solvent( } \mu L) \\
\text { (e.g. DMSO) }\end{array}$ & $\begin{array}{c}=\text { Intermediate } \\
\text { Concentration } \\
\left(\mathrm{mg} \cdot \mathrm{mL}^{-1}\right)\end{array}$ & $\begin{array}{c}\text { = Final } \\
\text { Concentration } \\
(\mu \mathrm{g} / \mathrm{mL})\end{array}$ & Log2 \\
\hline 1 & 160 & Stock & & & 160 & 1,600 & 4 \\
\hline 2 & 160 & Stock & 50 & 50 & 80 & 800 & 3 \\
\hline 3 & 160 & Stock & 50 & 150 & 40 & 400 & 2 \\
\hline 4 & 160 & Stock & 50 & 350 & 20 & 200 & 1 \\
\hline 5 & 20 & Step 4 & 50 & 50 & 10 & 100 & 0 \\
\hline 6 & 20 & Step 4 & 50 & 150 & 5 & 50 & -1 \\
\hline 7 & 20 & Step 4 & 50 & 350 & 2.5 & 25 & -2 \\
\hline 8 & 2.5 & Step 7 & 50 & 50 & 1.25 & 12.5 & -3 \\
\hline 9 & 2.5 & Step 7 & 50 & 150 & 6.25 & 6,25 & -4 \\
\hline 10 & 2.5 & Step 7 & 50 & 350 & 0.313 & 3.13 & -5 \\
\hline
\end{tabular}

*Amphotericin $B$ (stock $=1,600 \mu \mathrm{g} \cdot \mathrm{mL}^{-1}$ ) is diluted into 16 to $0.0313 \mu \mathrm{g} \cdot \mathrm{mL}^{-1}$ in inoculum. 


\section{Inoculation and Incubation}

1) Label 10 round-bottom, polystyrene, sterile tubes $(12 \times 75 \mathrm{~mm})$ from 1 to 10 corresponding to the chemical concentrations from stock to $2^{9}$ fold dilution;

2) Add $990 \mu \mathrm{L}$ of inoculum and $10 \mu \mathrm{L}$ of diluted to each tube except two controls:

- Growth control (GC): $990 \mu \mathrm{L}$ of inoculum $+10 \mu \mathrm{L}$ of DMSO;

- Sterile control (SC): $990 \mathrm{~mL}$ of RPMI-1640 + $10 \mu \mathrm{L}$ of DMSO.

3) Incubate the tubes and the PDA plate indicator at $35^{\circ} \mathrm{C}$, observe daily.

\section{MIC Determination: Colorimetric method}

1) Add $50 \mu \mathrm{L}$ of resazurin solution $\left(100 \mathrm{mg} \cdot \mathrm{L}^{-1}\right.$ in RPMI-1640 medium, membrane sterile, Sigma-Aldrich) to each tube after $48 \mathrm{hrs}$ of incubation or when there is visible growth on the PDA plate;

2) Further incubate for another 1 to 24 hrs until GC becomes complete pink;

3) Measure the absorption spectrum of the chemical-inoculum-combinations that remain blue or of indiscernible colour change from blue to bluish-purple (visually scored 0 and 1 as shown in Table A2) at wavelengths from 400 to $800 \mathrm{~nm}$; undyed RPMI-1640 medium is run as the baseline.

Table A2. Numerical scoring of visual examination

\begin{tabular}{|c|c|c|c|}
\hline Score & Turbidity & Colour change & Inhibition \\
\hline $\mathbf{0}$ & optically clear & blue & complete inhibition \\
$\mathbf{1}$ & $25 \%$ growth & bluish purple & strong inhibition \\
$\mathbf{2}$ & $50 \%$ growth & purple & prominent inhibition \\
$\mathbf{3}$ & $75 \%$ growth & reddish purple & slight inhibition \\
$\mathbf{4}$ & $100 \%$ growth & Pink & No inhibition \\
\hline
\end{tabular}




\section{References}

[1] G. J. Smith, R. Chadwick, N. Konese, S. Scheele, S. E. Tauwhare and R. J. Weston, Textiles and Text (Edited by M. Hayward and E. Kramer), Archetype Publications, London, 2007, 264-268.

[2] M. Evans and R. Ngarimu, The Art of Maori Weaving: The Eternal Thread / Te Aho Mutunga Kore Huia Publishers, Wellington, NZ, 2005.

[3] D. J. Carr, N. M. Cruthers, R. M. Laing and B. E. Niven, Textile Research Journal 2005, 75, 93-98.

[4] W. Harris, New Zealand Journal of Botany 2005, 43, 83-118.

[5] B. Lowe, D. J. Carr, R. E. McCallum, T. Myers, B. E. Niven, R. Cameron, A. Gorham, C. Holtham and K. Te Kanawa, the Combined (NZ and AUS) Conference of The Textile Institute (Dunedin, New Zealand) 2009.

[6] H. M. Mead, Te Whatu Taniko: Taniko Weaving - Technique and Tradition, Raupo Publishing Ltd, NZ, 2006.

[7] D. Te Kanawa, Weaving a Kakahu, Bridget Williams Books Ltd, Wellington, 1992.

[8] A. Clarke, The Great Sacred Forest of Tane, Raupo Publishing (NZ) Ltd, 2007.

[9] S. M. Scheele, Harakeke: The Rene Orchiston Collection, Manaaki Whenua Press, Lincoln, NZ, 2005.

[10] M. King, New Zealand Journal of Botany 2003, 41, 571-578.

[11] W. Harris and M. Woodcock-Sharp, New Zealand Journal of Botany 2000, 38, 469-487.

[12] Labillardière, Annales du Museum National d 'Histoire Naturelle 1803, 2, 474484 . 
[13] B. D. Cross, Transactions and Proceedings of the New Zealand Institute 1915, 47, 61-66.

[14] B. D. Cross, Investigations on Phormium with regard to the improvement of its economic importance, MA thesis University of Canterbury, Christchurch, NZ, 1912.

[15] M. J. King and J. F. V. Vincent, New Zealand Journal of Botany 1996, 263, 521527.

[16] W. Harris, S. M. Scheele, G. J. Forrester and D. A. Peltzer, New Zealand Journal of Botany 2005, 43, 791-816

[17] N. M. Cruthers, D. J. Carr, R. M. Laing and B. E. Niven, Textile Research Journal 2006, 76, 601-606.

[18] W. Harris, S. M. Scheele, G. J. Forrester, M. Murray, K. T. Kanawa and E. Pahewa, New Zealand Journal of Botany 2007, 45, 111-137.

[19] K. McBreen, P. J. Lockhart and P. A. McLenachan, New Zealand Journal of Botany 2003, 41, 301-310.

[20] R. D. Smissen and P. B. Heenan, New Zealand Journal of Botany 2007, 45, 419432.

[21] W. Harris, S. M. Scheele, G. J. Forrester, K. T. E. Kanawa, M. Murray and E. Pahewa, New Zealand Journal of Botany 2008, 46, 401-423.

[22] R. H. Newman, S. E. K. Tauwhare, S. M. Scheele and R. Te Kanawa, Holzforschung 2005, 59, 147-152.

[23] N. More, G. Smith, R. T. Kanawa and I. Miller, Dyes in History and Archaeology 2003, 19, 144-148.

[24] G. J. Smith, R. Te Kanawa, I. Miller and G. Fenton, Dyes in History and Archaeology 2005, 20, 89-94.

[25] G. J. Smith and R. Te Kanawa, Chemistry in New Zealand 2008, 72, 127-131.

[26] S. Brooker and R. Cooper, Economic Botany 1961, 15, 1-10.

[27] S. M. Kupchan, H. Meshulam and A. T. Sneden, Phytochemistry 1978, 17, 767769. 
[28] G. J. Gainsford, Acta Crystallographica Section C 1995, 51, 709-712.

[29] H. E. Harvey and J. M. Waring, Journal of Natural Products 1987, 50, 767-768.

[30] Papermaking Art and Craft, Library of Congress, Washington, D.C. US, 1968.

[31] P. Long and R. Levering, Paper Art and Technology: The History and Methods of Fine Papermaking with a Gallery of Contemporary Paper Art World Print Council, San Francisco, US, 1978.

[32] G. J. M. Goedvriend, Endeavour 1988, 12, 38-43.

[33] J. Needham and T.-H. Tsien, Science and Civilisation in China: Volume 5, Chemistry and Chemical Technology; Part 1, Paper and Printing, Cambridge University Press, UK, 1985.

[34] F. W. Tsai and D. van der Reyden, The paper conservator: journal of the Institute of Paper Conservation 1997, 21, 48-62.

[35] E. W. Haylock, Paper: Its Making Merchanting and Usage Longman, London, 1974.

[36] J. Su, Conservation of the papermaking crafts of Lianshi paper, MA thesis Fudan University, Shanghai, 2008.

[37] The traditional handicrafts of making Xuan paper, United Nations Educational, Scientific and Cultural Organization (UNESCO), 2009, http://www.unesco.org/culture/ich/index.php?RL=00201.

[38] X.-y. Fang, D.-t. Wu and Y.-k. Lu, Journal of Beijing Institute of Graphic Communication 2008, 16, 1-8.

[39] N. Na, Q.-m. Ouyang, H. Ma, J. Ouyang and Y. Li, Talanta 2004, 64, 1000-1008. [40] R. J. H. Clark, P. J. Gibbs, K. R. Seddon, N. M. Brovenko and Y. A. Petrosyan, Journal of Raman Spectroscopy 1997, 28, 91-94.

[41] S. E. J. Bell, E. S. O. Bourguignon, A. C. Dennis, J. A. Fields, J. J. McGarvey and K. R. Seddon, Analytical Chemistry 1999, 72, 234-239.

[42] P. J. Gibbs, K. R. Seddon, N. M. Brovenko, Y. A. Petrosyan and M. Barnard, Analytical Chemistry 1997, 69, 1965-1969. 
[43] X.-c. Li, Journal of Guangxi University for Nationalities (Natural Science Edition) 2009, 15, 59-63.

[44] T. Li, Y. Wu, Y.-F. Shie, T.-S. Wang and S.-Y. Fang, Journal of cultural property conservation 2009, 8, 40-47.

[45] W.-j. Xu, Sciences of Conservation and Archaeology 2008, 20, 47-50.

[46] W.-j. Xu and Y.-s. Chen, Sciences of Conservation and Archaeology 2007, 19, $20-$ 25.

[47] K.-T. Wang and I.-C. Wang, Taiwan Linye Kexue 2001, 14, 227-236.

[48] C. K. Min, J. Y. Jo and S. H. Lee, Journal of Korea Technical Association of the Pulp and Paper Industry 2000, 32, 81-86.

[49] K.-T. Wang, E. I.-C. Wang and Y.-S. Perng, Taiwan Linye Kexue 2008, 23, 155164.

[50] T.-C. Shiah, T.-T. Chang and C.-H. Fu, Taiwan Journal of Forest Science 2001, 16, 327-332.

[51] L.-D. Lin, C.-M. Hsieh, B.-H. Chiang and M.-J. Tsai, Journal of Wood Science 2007, 53, 121-126.

[52] J. Szostak-Kotowa, International Biodeterioration and Biodegradation 2004, 53, $165-170$.

[53] D. Montegut, N. Indictor and R. J. Koestler, International Biodeterioration 1991, $28,209-226$.

[54] M.-L. E. Florian, Fungal Facts: Solving fungal problems in heritage collections, Archetype Publications, 2002.

[55] P. Tiano, Biodegradation of cultural heritage: decay mechanisms and control method, .Ninth ARIADNE Workshop "Historic Material and their Diagnostic", Advanced Research Centre for Cultural Heritage Interdisciplinary Projects, Prague, 2002.

[56] M. P. Nugari, G. F. Priori, D. Mat and F. Scala, International Biodeterioration 1987, 23, 295-306.

[57] R. J. Grayer and J. B. Harborne, Phytochemistry 1994, 37, 19-42. 
[58] R. J. Grayer and T. Kokubun, Phytochemistry 2001, 56, 253-263.

[59] A. C. Stein, S. Álvarez, C. Avancini, S. Zacchino and G. von Poser, Journal of Ethnopharmacology 2006, 107, 95-98.

[60] T. Ojala, S. Remes, P. Haansuu, H. Vuorela, R. Hiltunen, K. Haahtela and P. Vuorela, Journal of Ethnopharmacology 2000, 73, 299-305.

[61] C. L. Cespedes, J. G. Avila, A. Martinez, B. Serrato, J. C. Calderon-Mugica and R. Salgado-Garciglia, Journal of Agribultural and Food Chemistry 2006, 54, 35213527.

[62] J. J. Kim, S. Ben-Yehoshua, B. Shapiro, Y. Henis and S. Carmeli, Plant Physiology 1991, 97, 880-885.

[63] F. M. Al-Barwani and E. A. Eltayeb, Biochemical Systematics and Ecology 2004, 32, 1097-1108.

[64] Personal Communication with Landcare Research Ltd., Auckland, 2007.

[65] Reference method for broth dilution antifungal susceptibility testing of filamentous fungi: approved standard., National Committee for Clinical Laboratory Standards, Wayne, PA, 2002.

[66] W. Rasband, ImageJ, U. S. National Institutes of Health, Bethesda, Maryland, USA, 1997-2005, http://rsb.info.nih.gov/ij/.

[67] G. Carpentier, Dot Blot Analyzer for ImageJ - Documentation, http://image.bio.methods.free.fr/dotblot.html.

[68] A. Espinel-Ingroff, A. Fothergill, J. Peter, M. G. Rinaldi and T. J. Walsh, Journal of Clinical Microbiology 2002, 40, 3204-3208.

[69] S. Sardari, Y. Mori, K. Horita, R. G. Micetich, S. Nishibe and M. Daneshtalab, Bioorganic and Medicinal Chemistry 1999, 7, 1933-1940.

[70] M. C. Carpinella, C. G. Ferrayoli and S. M. Palacios, Journal of Agricultural and Food Chemistry 2005, 53, 2922-2927.

[71] E. d. Hoffmann and V. Stroobant, Mass spectrometry: principles and applications, John Wiley and Sons, Ltd., Chichester, UK, 2007.

[72] I. Manisali, D. D. Y. Chen and B. B. Schneider, TrAC Trends in Analytical 
Chemistry 2006, 25, 243-256.

[73] W. F. Smyth, S. McClean, C. J. Hack, V. N. Ramachandran, B. Doherty, C. Joyce, F. O'Donnell, T. J. Smyth, E. O'Kane and P. Brooks, Trends in Analytical Chemistry 2006, 25, 572-582.

[74] N. S. Vul'fson, V. I. Zaretskii and V. G. Zaikin, Russian Chemical Bulletin 1963, 12, 2046-2049.

[75] S. Concannon, V. N. Ramachandran and W. F. Smyth, Rapid Communications in Mass Spectrometry 2000, 14, 2260-2270.

[76] H. Johnson, Vintage: The Story of Wine, Simon \& Schuster, New York, US, 1989.

[77] C. H. J. Wells, Introduction to Molecular Photochemistry, Chapman \& Hall, London, UK, 1972.

[78] A. Gilbert and J. Baggott, Essentials of Molecular Photochemistry, Blackwell Scientific Publications, Oxford, UK, 1991.

[79] H. Zollinger, Color Chemistry: Syntheses, Properties and Applications of Organic Dyes and Pigments, Wiley-VCH Verlag GmbH, Weinheim, 2003.

[80] S. R. Trenor, A. R. Shultz, B. J. Love and T. E. Long, Chemical Reviews 2004, 104, 3059-3077.

[81] B. Valeur, Molecular Fluorescence: Principles and Applications Wiley-VCH Verlag GmbH, Weinheim, 2001.

[82] L. A. Holt, I. H. Leaver and B. Milligan, Textile Research Journal 1976, 46, 539544 .

[83] C. Gloxhuber and H. Bloching, Clinical Toxicology 1978, 13, 171-203.

[84] S. Sardari, S. Nishibe and M. Daneshtalab, Studies in Natural Products Chemistry 2000, 23, 335-393.

[85] G. J. Smith, I. J. Miller and V. Daniels, Journal of Photochemistry and Photobiology A: Chemistry 2005, 169, 147-152.

[86] A. A. da Silva, E. S. P. do Nascimento, D. R. Cardoso and D. W. Franco, Journal of Separation Science 2009, 32, 3681-3691. 
[87] D. W. Fink and W. R. Koehler, Analytical Chemistry 1970, 42, 990-993.

[88] A. M. Ferrari, M. Sgobba, M. C. Gamberini and G. Rastelli, European Journal of Medicinal Chemistry 2007, 42, 1028-1031.

[89] G. J. Smith, Journal of Photochemistry and Photobiology B: Biology 1995, 27, 187198.

[90] R. S. Davidson, Journal of Photochemistry and Photobiology B: Biology 1996, 33, $3-25$.

[91] N. Lucas, C. Bienaime, C. Belloy, M. Queneudec, F. Silvestre and J. E. NavaSaucedo, Chemosphere 2008, 73, 429-442.

[92] H. Tylli, I. Forsskåhl and C. Olkkonen, Journal of Photochemistry and Photobiology A: Chemistry 1993, 76, 143-149.

[93] C. Noutary, P. de Violet, J. Vercauteren and A. Castellan, Research on Chemical Intermediates 1995, 21, 223-245.

[94] C. Heitner, Photochemistry of Lignocellulosic Materials 1993, ACS Symposium Series 531, 2-25.

[95] G. Gellerstedt and E. Pettersson, Acta Chemica Scandinavica B 1975, 29, 10051010.

[96] I. H. Leaver, Photochemistry and Photobiology 1978, 27, 451-456.

[97] K. R. Millington and G. Maurdev, Journal of Photochemistry and Photobiology A: Chemistry 2004, 165, 177-185.

[98] K. Apel and H. Hirt, Annual Review of Plant Biology 2004, 55, 373-399.

[99] I. Heiser, W. Oszwald and E. F. Elstner, Plant Physiology and Biochemistry 1998, 36, 703-713.

[100] D. T. Sawyer, M. J. Gibian, M. M. Morrison and E. T. Seo, Journal of the American Chemical Society 1978, 100, 627-628.

[101] B. Halliwell and J. M. Gutteridge, Archives of biochemistry and biophysics 1986, $246,501-514$.

[102] A. d. Silva, E. d. Nascimento, D. Cardoso and D. Franco, Journal of Separation 
Science 2009, 32, 3681-3691.

[103] T. Smyth, V. N. Ramachandran and W. F. Smyth, International Journal of Antimicrobial Agents 2009, 33, 421-426.

[104] C. J. Douglas, Trends in Plant Science 1996, 1, 171-178.

[105] K. Kai, B.-i. Shimizu, M. Mizutani, K. Watanabe and K. Sakata, Phytochemistry 2006, 67, 379-386.

[106] T. Vogt, Molecular Plant 2010, 3, 2-20.

[107] F. Bourgaud, A. Hehn, R. Larbat, S. Doerper, E. Gontier, S. Kellner and U. Matern, Phytochemistry Reviews 2006, 5, 293-308.

[108] A. Vianello and F. Macri, Journal of Bioenergetics and Biomembranes 1991, 23, 409.

[109] K. D. Belfield, M. V. Bondar, Y. Liu and O. V. Przhonska, Journal of Physical Organic Chemistry 2003, 16, 69-78.

[110] S. C. Shim, B. M. Jeong and Y. H. Paik, The Bulletin of the Korean Chemical Society 1992, 13, 684-688.

[111] M. D'Auria and R. Racioppi, Journal of Photochemistry and Photobiology A: Chemistry 2004, 163, 557-559.

[112] K. Gnanaguru, N. Ramasubbu, K. Venkatesan and V. Ramamurthy, The Journal of Organic Chemistry 1985, 50, 2337-2346.

[113] J. N. Moorthy, K. Venkatesan and R. G. Weiss, The Journal of Organic Chemistry 1992, 57, 3292-3297.

[114] R. J. von Trebra and T. H. Koch, Journal of Photochemistry 1986, 35, 33-46.

[115] G. Jones Ii, W. R. Bergmark and W. R. Jackson, Optics Communications 1984, $50,320-323$.

[116] X. Yu, D. Scheller, O. Rademacher and T. Wolff, The Journal of Organic Chemistry 2003, 68, 7386-7399.

[117] Z.-Y. Jiang, A. C. S. Woollard and S. P. Wolff, FEBS Letters 1990, 268, 69-71.

[118] M. Linetsky and B. J. Ortwerth, Journal of Bioenergetics and Biomembranes 
$1995,62,87-93$.

[119] M. Linetsky, H. L. James and B. J. Ortwerth, Experimental Eye Research 1996, $63,67-74$.

[120] K. Bredereck and C. Schumacher, Dyes and Pigments 1993, 23, 135-147.

[121] J. Kagan, Journal of the American Chemical Society 1966, 88, 2617-2618.

[122] A. U. Khan, The Journal of Physical Chemistry 1976, 80, 2219-2228.

[123] D. Rehm and A. Weller, Israel Journal of Chemistry 1970, 8, 259-271.

[124] G. Kavarnos, Fundamental Concepts of Photoinduced Electron Transfer (Edited by J. Mattay), Springer-Verlag, Berlin Heidelberg, 1990, 21-58.

[125] T. Moriya, Bulletin of the Chemical Society of Japan 1988, 61, 1873-1886

[126] J. Seixas de Melo and P. F. Fernandes, Journal of Molecular Structure 2001, 565-566, 69-78.

[127] G. J. Smith, C. L. Dunford and P. B. Roberts, Journal of Photochemistry and Photobiology A: Chemistry 2010, 210, 31-35.

[128] C. P. Andrieux, P. Hapiot and J. M. Saveant, Journal of the American Chemical Society 1987, 109, 3768-3775.

[129] H. Morrison, H. Curtis and T. McDowell, Journal of the American Chemical Society 1966, 88, 5415-5419.

[130] J. M. Dyer, C. D. Cornellison, S. D. Bringans, G. Maurdev and K. R. Millington, Photochemistry and Photobiology 2008, 84, 145-153.

[131] H. A. Carter, Journal of Chemical Education 1996, 73, 417-420.

[132] M. Strlic, J. Thomas, T. Trafela, L. Cséfalvayová, I. Kralj Cigić, J. Kolar and M. Cassar, Analytical Chemistry 2009, 81, 8617-8622.

[133] J. F. Waterhouse, Paper Preservation Symposium (Washington, DC) 1988, 5963

[134] H. A. Carter, Journal of Chemical Education 1996, 73, 1068-1073.

[135] M. Missori, M. Righini and S. Selci, Optics Communications 2004, 231, 99-106. 
[136] M. J. John and S. Thomas, Carbohydrate Polymers 2008, 71, 343-364.

[137] T. D. Barrett and J. F. Waterhouse, Tappi Journal 1991, 74, 207-212.

[138] H. Arai, International Biodeterioration and Biodegradation 2000, 46, 181-188.

[139] M. Missori, M. Righini and A.-L. Dupont, Optics Communications 2006, 263, 289-294.

[140] S. B. Lee, J. Bogaard and R. L. Feller, ACS Symposium Series 1989, 410, 54-62.

[141] M. L. E. Florian and L. Manning, International Biodeterioration and Biodegradation 2000, 46, 205-220.

[142] R. Yeat, Unpublished Work, 2008.

[143] G. Righini, A. L. Segre, G. Mattogno, C. Frederici and P. F. Munafo, Naturwissenschaften 1998, 85, 171-175.

[144] L. Laguardia, E. Vassallo, F. Cappitelli, E. Mesto, A. Cremona, C. Sorlini and G. Bonizzoni, Applied Surface Science 2005, 252, 1159-1166.

[145] M. Bicchieri, A. Sodo, G. Piantanida and C. Coluzza, Journal of Raman Spectroscopy 2006, 37, 1186-1192.

[146] M. Manso, S. Pessanha and M. L. Carvalho, Spectrochimica Acta Part B: Atomic Spectroscopy 2006, 61, 922-928.

[147] I. Viola, S. Bubici, C. Casieri and F. De Luca, Journal of Cultural Heritage 2004, $5,257-261$.

[148] C. Casieri, S. Bubici, I. Viola and F. De Luca, Solid State Nuclear Magnetic Resonance 2004, 26, 65-73.

[149] M. Manso and M. L. Carvalho, Spectrochimica Acta Part B: Atomic Spectroscopy 2009, 64, 482-490.

[150] A. M. Corte, A. Ferroni and V. S. Salvo, International Biodeterioration and Biodegradation 2003, 51, 167-173.

[151] F. Pinzari, G. Pasquariello and A. De Mico, Macromolecular Symposia 2006, $238,57-66$.

[152] F. Pinzari, M. Zotti, A. De Mico and P. Calvini, International Biodeterioration 
and Biodegradation 2010, 64, 499-505.

[153] H. G. M. Edwards, The Analyst 2004, 129, 870-879.

[154] M. Zotti, A. Ferroni and P. Calvini, International Biodeterioration and Biodegradation 2008, 62, 186-194.

[155] M. Manso, S. Pessanha, F. Figueira, S. Valadas, A. Guilherme, M. Afonso, A. Rocha, M. Oliveira, I. Ribeiro and M. Carvalho, Analytical and Bioanalytical Chemistry 2009, 395, 2029-2036.

[156] The Representative List of the Intangible Cultural Heritage of Humanity, United Nations Educational, Scientific and Cultural Organization (UNESCO), 2009.

[157] N. Kohara, C. Sano, H. Ikuno, Y. Magoshi, M. A. Becker, M. Yatagai and M. Saito, Historic Textiles, Papers, and Polymers in Museums, American Chemical Society, 2000, 74-85.

[158] T. Bechtold, A. Turcanu, E. Ganglberger and S. Geissler, Journal of Cleaner Production 2003, 11, 499-509.

[159] A. K. Sarkar and C. M. Seal, Clothing and Textiles Research Journal 2003, 21, $162-166$.

[160] H. T. Deo and B. K. Desai, Coloration Technology 1999, 115, 224-227.

[161] E. Yi and J.-Y. Cho, Color Research and Application 2008, 33, 148-157.

[162] D. Cardon, Natural Dyes: Sources, Tradition, Technology and Science, Archetype Publications, London, 2007.

[163] G. W. Taylor, Dyes in History and Archaeology 2002, 18, 37-39.

[164] D. G. Duff and R. S. Sinclair., Dyes in History and Archaeology 1988, 7, 25-31.

[165] J. Prousek, Pure and Applied Chemistry 2007, 79, 2325-2338.

[166] K. E. Hammel, A. N. Kapich, K. A. J. Jr. and Z. C. Ryan, Enzyme and Microbial Technology 2002, 30, 445-453.

[167] R. Stephen Davidson, L. A. Dunn, A. Castellan and A. Nourmamode, Journal of Photochemistry and Photobiology A: Chemistry 1991, 58, 349-359.

[168] K. R. Millington, Coloration Technology 2006, 122, 301-316. 
[169] D. Cristea and G. Vilarem, Dyes and Pigments 2006, 70, 238-245.

[170] I. J. Miller and G. J. Smith, Journal of the Society of Dyers and Colourists 1995, 111, 103-106.

[171] Y.-H. Lee and H.-D. Kim, Fibers and Polymers 2004, 5, 303-308.

[172] R. T. Kanawa, S. Thomsen, G. Smith, I. Miller, C. Andary and D. Cardon, Dyes in History and Archaeology 2002, 18, 47-50.

[173] D. M. Pearsall, American Anthropologist 1982, 84, 862-871.

[174] A. G. Sangster, M. J. Hodson and H. J. Tubb, Studies in Plant Science 2001, 8, $85-113$.

[175] A. Adriaens, Spectrochimica Acta Part B: Atomic Spectroscopy 2005, 60, 15031516.

[176] E. Bulska, B. Wagner and M. G. Sawicki, Microchimica Acta 2001, 136, 61-66.

[177] R. Giorgi, L. Dei, M. Ceccato, C. Schettino and P. Baglioni, Langmuir 2002, 18, 8198-8203.

[178] J. Goldstein, D. E. Newbury, D. C. Joy, C. E. Lyman, P. Echlin, E. Lifshin, L. Sawyer and J. R. Michael, Scanning Electron Microscopy and X-ray Microanalysis, Kluwer Academic, Plenum Publishers, New York, 2003.

[179] B. Beckhoff, B. Kanngießer, N. Langhoff, R. Wedell and H. Wolff, Handbook of Practical X-Ray Fluorescence Analysis, Springer-Verlag Berlin Heidelberg, 2006.

[180] Amptek X-ray emission $K \mathcal{E} L$ line lookup chart, Amptek Inc. http://www.amptek.com/xray_chart.html.

[181] G. McMahon, Analytical Instrumentation: A Guide to Laboratory, Portable and Miniaturized Instruments, John Wiley and Sons Ltd., Chichester, UK , 2007.

[182] A. Montaser, Inductively Coupled Plasma Mass Spectrometry, Wiley-VCH, New York, 1998.

[183] C. J. Morris, J. R. Earl, C. W. Trenam and D. R. Blake, The International Journal of Biochemistry and Cell Biology 1995, 27, 109-122.

[184] A. S. Polla, L. L. Polla and B. S. Polla, Ageing Research Reviews 2003, 2, 25-37. 
[185] R. Agnemo, R. C. Francis, T. C. Alexander and C. W. Dence, Holzforschung 1991, 45, 101-108.

[186] C. W. Trenam, D. R. Blake and C. J. Morris, Journal of Investigative Dermatology 1992, 99, 675-682.

[187] M. L. Circu and T. Y. Aw, Free Radical Biology and Medicine 2010, 48, 749-762.

[188] H. F. Poon, V. Calabrese, G. Scapagnini and D. A. Butterfield, Clinics in Geriatric Medicine 2004, 20, 329-359.

[189] M. Comporti, C. Signorini, G. Buonocore and L. Ciccoli, Free Radical Biology and Medicine 2002, 32, 568-576.

[190] S. Puntarulo, Molecular Aspects of Medicine 2005, 26, 299-312.

[191] J. M. De Freitas and R. Meneghini, Mutation Research/Fundamental and Molecular Mechanisms of Mutagenesis 2001, 475, 153-159.

[192] G. Cairo, S. Recalcati, A. Pietrangelo and G. Minotti, Free Radical Biology and Medicine 2002, 32, 1237-1243.

[193] K. Honda, G. Casadesus, R. B. Petersen, G. Perry and M. A. Smith, Annals of the New York Academy of Sciences 2004, 1012, 179-182.

[194] G. A. Qureshi, S. A. Syed, S. H. Parvez, G. A. Qureshi and S. H. Parvez, Oxidative Stress and Neurodegenerative Disorders, Elsevier Science B.V., Amsterdam, 2007, 719-735.

[195] G. J. Brewer, Experimental Biology and Medicine 2007, 232, 323-335.

[196] X. Huang, Mutation Research/Fundamental and Molecular Mechanisms of Mutagenesis 2003, 533, 153-171.

[197] J.-F. Briat, C. Duc, K. Ravet and F. Gaymard, Biochimica et Biophysica Acta (BBA) - General Subjects 2010, 1800, 806-814.

[198] F. S. Dobson, Lichens, an Illustrated Guide to the British and Irish Species., Richmond Publishing Co. Ltd., Slough, UK, 2000.

[199] V. Ahmadjian, The Lichen Symbiosis, John Wiley and Sons, Inc., New York, 1993. 
[200] O. W. Purvis and C. Halls, The Lichenologist 1996, 28, 671-601.

[201] P. L. Nimis, S. Andreussi and E. Pittao, The Science of The Total Environment 2001, 275, 43-51.

[202] L. Y. Foo, Phytochemistry 1987, 26, 2825-2830.

[203] L. Y. Foo, Phytochemistry 1989, 28, 2477-2481.

[204] K. R. Markham, Techniques of Flavonoid Identification, Academic Press, London, 1982. 